\title{
A RHEO-OPTIC STUDY OF WORMLIKE MICELLES SOLUTIONS
}

\author{
Allan Raudsepp \\ A thesis submitted to the Victoria University of Wellington \\ in fulfilment of the requirement for the degree of \\ Doctor of Philosophy \\ in Physics
}

Victoria University of Wellington

2009 


\section{Abstract}

Shear banding, where a fluid spatially partitions into strain rate or shear bands in steadystate simple shear flow conditions, was first observed in wormlike micelles solutions and has since been observed in many other complex fluids. These solutions have been used extensively to explore the relationship between shear (or stress) banding and microstructure in complex fluids. This relationship is difficult to study because of its dynamic nature and there is still no clear consensus as to how banding relates to microstructural changes in wormlike micelles solutions. In this thesis, the rheology of a number of wormlike micelles solutions is examined using both conventional and novel techniques with the view to developing a better understanding of this relationship.

The rheology of three wormlike micelles solutions composed of a surfactant cetylpyridinium chloride $(\mathrm{CPCl})$ and counterion sodium salicylate in water with or without the salt sodium chloride were examined using mechanical rheometry and the rheo-optical techniques: homodyne photo-correlation spectroscopy (PCS), diffusing wave spectroscopy (DWS) and ellipsometry. Rheo-mechanical measurements were largely consistent with the predictions of the reptation-reaction model. While significant stress fluctuations were noted in one particular flow geometry, they were generally not observed in most rheomechanical measurements presented here, indicating that these fluctuations are not universal and that they are geometry dependent. Shear induced turbidity was directly observed in the cone-plate and parallel-plate geometries with turbid rings forming in samples that showed a stress plateau. The Poisson-renewal model, which extends the reptationreaction model to include the influence of high frequency modes on the linear rheology, was tested experimentally using mechanical rheometry, DWS microrheology and literature data. In most cases the data fitted the model behaviour quite well, giving a physically reasonable estimate of the average length of the micelles. DWS's spatial sensitivity to shear induced relative motion was then used to probe the flow behaviour of selected wormlike micelles solutions in the cylindrical-Couette, cone-plate and parallel-plate geometries. In the cylindrical-Couette, the 'flow-DWS' measurements were largely consistent with rheo-mechanical measurements and indicated that some wormlike micelles solutions were partitioning into apparently stable high and low strain rate bands in the vicinity of the stress plateau. While measurements in the cone-plate and parallel-plate geometries also suggested shear banding in samples that showed a stress plateau, the interpretation was less clear-cut. Homodyne PCS was combined with ellipsometry to examine the spatial relationship between strain rate and birefringence banding in selected wormlike micelles 
solutions in a cylindrical-Couette geometry. In contrast to the observations of previous workers, it was found here that the birefringence and strain rate bands did coincide. Furthermore, the high strain rate band was observed to be more turbid than the lower strain rate band suggesting a connection between strain rate, optical anisotropy and turbidity. 


\section{Acknowledgements}

I gratefully thank my principal supervisor, Prof Paul Callaghan without whose encouragement, patience and support this thesis would not have been possible. I am also grateful to co-supervisors, Dr Yacine Hemar, Emer Prof David Beaglehole and Prof Pablo Etchegoin for their insights and advice.

Thanks to my fellow PhD students: Lauren Burcaw, Bradley Douglas, Meghan Halse, Mark Hunter, Dr Antoine Lutti, Simon Rogers, Hui Lin Tan and Dr Kate Washburn and resident Post-doctoral fellows: Dr Jen Brown, Dr Holger Eggert, Dr Kirk Feindel, Dr Petrik Galvosas, Dr Guillaume Madelin and Dr Ying Qiao who helped keep things fun and interesting during my $\mathrm{PhD}$.

I enjoyed many thoughtful (and sometimes intimidating) conversations with Assoc Prof Kate McGrath and frequent visitors, Dr Lourdes de Vargas and Dr Suzanne Fielding.

Thanks to Alan Rennie and Manu Pouajen-Blakiston for help with the design and for construction of the various rheo-optic geometries. I also thank Margaret Brown for her administrative support, and Rob Lenihan and Scott Forbes for their computer support.

I am indebted to Beaglehole Instruments Ltd. for loaning me the ellipsometer used in Chapter 8.

Finally, I would like to thank my partner for everything. 


\section{Contents}

$\begin{array}{ll}\text { Abstract } & \text { i }\end{array}$

Acknowledgements ii

Contents

List of figures viii

List of tables $\quad$ xiii

$\begin{array}{lll}1 & \text { Thesis overview } & 1\end{array}$

\begin{tabular}{|ll|l}
\hline 2 & Rheology & 5
\end{tabular}

2.1 Introduction . . . . . . . . . . . . . . . . . 5

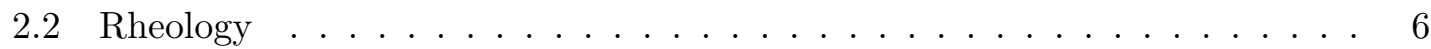

2.2 .1 Flow, stress and the constitutive relation $\ldots \ldots \ldots$. . . . . . 6

2.2 .2 Simple shear flow . . . . . . . . . . . . . . . . 6

$2.2 .3 \quad$ Linear rheology $\ldots \ldots \ldots$. . . . . . . . . . . . . . . . 10

2.2 .4 Nonlinear rheology $\ldots \ldots \ldots$. . . . . . . . . . . . . . 10

2.2 .5 Mechanical rheometry . . . . . . . . . . . . . . . . 11

2.3 Brownian motion and diffusion $\ldots \ldots \ldots \ldots \ldots \ldots$

2.4 The rheology of polymer-like chains $\ldots \ldots \ldots \ldots \ldots$

2.4 .1 Structure . . . . . . . . . . . . . . . . . . . 18

2.4 .2 Rubbery- and temporary-network models . . . . . . . . . . . . 18

2.4 .3 Reptation model . . . . . . . . . . . . . . . . . 20 
2.4 .4 Other relaxation modes $\ldots \ldots \ldots \ldots$. . . . . . . . . 23

\begin{tabular}{|ll|}
\hline 3 & Rheology of wormlike micelles solutions \\
\hline
\end{tabular}

3.1 Introduction . . . . . . . . . . . . . . . . . . . 27

3.2 Surfactants in solution . . . . . . . . . . . . . . . . . . . 27

3.3 Linear rheology of wormlike micelles $\ldots \ldots$. . . . . . . . . . . . . . 29

3.3 .1 Reptation-reaction model . . . . . . . . . . . . . . . . . 29

3.3 .2 Poisson-renewal model . . . . . . . . . . . . . . . . . . . 31

3.4 Nonlinear rheology of wormlike micelles . . . . . . . . . . . . . . . 34

3.4 .1 Shear banding . . . . . . . . . . . . . . . . . . 34

3.4 .2 Coupling between flow and concentration . . . . . . . . . . . . 38

3.4 .3 Stress fluctuations and flow instabilities . . . . . . . . . . . . . 40

\begin{tabular}{|ll|}
\hline 4 & Rheo-optics \\
\hline
\end{tabular}

4.1 Introduction . . . . . . . . . . . . . . . . . . . . . . . . . 45

4.2 Solving Maxwell equations . . . . . . . . . . . . . . . . . . . . . . . 46

$4.2 .1 \quad$ Maxwell equations . . . . . . . . . . . . . . . . . 46

4.2 .2 Plane wave solution $\ldots \ldots \ldots$. . . . . . . . . . . . 46

4.2 .3 Green function solution . . . . . . . . . . . . . . . . 47

4.3 Propagation in homogeneous media . . . . . . . . . . . . . . . . . . . 48

$4.3 .1 \quad$ Propagation in isotropic materials . . . . . . . . . . . . . . 48

$4.3 .2 \quad$ Propagation in anisotropic material $\ldots \ldots \ldots$. . . . . . . . 48

4.3 .3 Matrix formulations . . . . . . . . . . . . . . . 50

4.4 Propagation in inhomogeneous media . . . . . . . . . . . . . . . 52

$4.4 .1 \quad$ Single scattering regime $\ldots \ldots \ldots \ldots$. . . . . . . . . . . 52

4.4 .2 Multiple scattering in the diffusion approximation . . . . . . . . 57

4.4 .3 Matrix formulations . . . . . . . . . . . . . . . . 61

$4.4 .4 \quad$ Scattering birefringence and dichroism . . . . . . . . . . . . 63

4.5 Rheo-optical properties of polymer like systems . . . . . . . . . . . . . . 64

\begin{tabular}{|lll}
5 & Rheometry and visualisation & 69
\end{tabular}

5.1 Introduction . . . . . . . . . . . . . . . . . . . . . . . . . 69 
5.2 Experimental $\ldots \ldots \ldots \ldots \ldots \ldots \ldots$

5.3 Measurements and analysis $\ldots \ldots \ldots \ldots$. . . . . . . . . . 70

$5.3 .1 \quad$ Linear rheology . . . . . . . . . . . . . . . . . . . . 70

5.3 .2 Nonlinear rheology . . . . . . . . . . . . . . . . . . 75

5.3 .3 Direct observation . . . . . . . . . . . . . . . . 82

5.4 Discussion . . . . . . . . . . . . . . . . . . . . 90

$\begin{array}{lll}6 & \text { Poisson-renewal model } & 93\end{array}$

6.1 Introduction . . . . . . . . . . . . . . . . . . . . 93

6.2 Microrheology . . . . . . . . . . . . . . . . . . . . . . 93

6.3 Measurements and analysis $\ldots \ldots \ldots \ldots \ldots$. . . . . . . . . . 95

6.3 .1 Microrheology . . . . . . . . . . . . . . . . . . 95

6.3 .2 Mechanical rheometry . . . . . . . . . . . . . . . . . 99

6.3 .3 Analysis . . . . . . . . . . . . . . . . . . 101

6.4 Diffusion NMR microrheology $\ldots$. . . . . . . . . . . . . . . . . . . . . . . . . 109

6.5 Discussion . . . . . . . . . . . . . . . . . . . . . . 112

\begin{tabular}{lll}
\hline & Flow-DWS & 115
\end{tabular}

7.1 Introduction . . . . . . . . . . . . . . . . . . . . . 115

7.2 DWS and flow . . . . . . . . . . . . . . . . . 115

7.3 Experimental . . . . . . . . . . . . . . . . . . . . . . . . . . . 119

7.3 .1 Set-up . . . . . . . . . . . . . . . . . . 119

$7.3 .2 \quad$ Samples . . . . . . . . . . . . . . . . . . . . 122

7.4 Measurements and analysis . . . . . . . . . . . . . . . . 123

7.4 .1 Cylindrical-Couette geometry . . . . . . . . . . . . . . 123

$7.4 .2 \quad$ Cone-plate geometry . . . . . . . . . . . . . . . . . . . . 129

$7.4 .3 \quad$ Parallel-plate geometry . . . . . . . . . . . . . . . 135

7.5 Discussion . . . . . . . . . . . . . . . . . 137

8 Birefringence and shear banding 141

8.1 Introduction . . . . . . . . . . . . . . . . . . . . . . . . . . 141

8.2 Experimental . . . . . . . . . . . . . . . . . . . . . . . 142 
8.2 .1 Set-up . . . . . . . . . . . . . . . . . . . . . 142

8.2 .2 Samples . . . . . . . . . . . . . . . . . . . . 144

$8.2 .3 \quad$ Homodyne photo-correlation spectroscopy . . . . . . . . . . . . . . . 145

8.2 .4 Ellipsometry . . . . . . . . . . . . . . . . . . 155

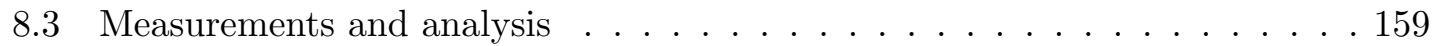

8.3 .1 Homodyne PCS . . . . . . . . . . . . . . . . . . . . 159

$8.3 .2 \quad$ Ellipsometry . . . . . . . . . . . . . . . . . 164

8.4 Discussion . . . . . . . . . . . . . . . . . . 170

\begin{tabular}{lll}
\hline 9 & Simulating dynamic speckle & 173
\end{tabular}

9.1 Introduction . . . . . . . . . . . . . . . . . . . . . . . . . . 173

9.2 Statistical properties of dynamic speckle $\ldots \ldots$. . . . . . . . . . 175

9.3 Experimental . . . . . . . . . . . . . . . . . . . . . . . 178

9.4 Measurements and analysis $\ldots \ldots \ldots \ldots$. . . . . . . . . . . . 180

9.4 .1 Translation . . . . . . . . . . . . . . . . . . . . 180

9.4 .2 Simple shear flow . . . . . . . . . . . . . . . . . . . . 184

9.5 Discussion . . . . . . . . . . . . . . . . . . . . . . . . . 185

$\begin{array}{ll}10 \text { Concluding remarks } & 189\end{array}$

10.1 Summary . . . . . . . . . . . . . . . . . . . . . . . . 189

10.2 Flow-concentration coupling? . . . . . . . . . . . . . . . . . . 191

10.3 Future work . . . . . . . . . . . . . . . . . . . . 193 


\section{List of Figures}

2.1 Physical appearance of wormlike micelles solutions $\ldots \ldots \ldots$. . . . . . 5

2.2 Simple shear flow geometry $\ldots \ldots \ldots \ldots \ldots$. . . . . . . . . . 7

2.3 Spring-dashpot mechanical circuit analogues used in rheology . . . . . . . . 9

2.4 Flow curves of common fluids . . . . . . . . . . . . . . . . . . . 11

2.5 Standard flow geometries used in rheometry . . . . . . . . . . . . 12

2.6 Simulated Brownian motion of a Kelvin element . . . . . . . . . . . . . . . 15

2.7 Simulated Brownian motion in restricted geometries . . . . . . . . . . . 17

$2.8 \quad$ End-to-end displacement vector $\mathbf{R} \ldots \ldots \ldots \ldots$

$2.9 \quad$ Stress and deformation of a rubbery-network $\ldots \ldots \ldots \ldots$

2.10 Topological constraints on an entangled polymer . . . . . . . . . . . . 21

2.11 Linear rheology of an reptating polymer . . . . . . . . . . . . . . . 22

2.12 Nonlinear rheology of an reptating polymer . . . . . . . . . . . . . . . 23

2.13 High frequency relaxation modes . . . . . . . . . . . . . . 25

3.1 Surfactants in solution . . . . . . . . . . . . . . . . . . . . . . . . . . . . . . 29

3.2 Maxwell-like response of a wormlike micelles solution . . . . . . . . . . . . 30

3.3 Evolution of the primitive path according to the reptation-reaction and Poisson-renewal models . . . . . . . . . . . . . . . . 31

3.4 Linear rheology predicted by the reptation-reaction model . . . . . . . . . . 32

3.5 Linear rheology predicted by the Poisson-renewal model . . . . . . . . . . . 33

3.6 Testing the Poisson-renewal model . . . . . . . . . . . . . . . . . 34

3.7 Nonlinear rheology predicted by the reptation-reaction model . . . . . . . . 35

3.8 Optical anisotropy, shear banding and turbidity observed in sheared worm- 
3.9 Gradient and vorticity banding . . . . . . . . . . . . . . . . . 40

3.10 Sigmoidal stress relaxation $\ldots \ldots \ldots \ldots$. . . . . . . . . . . . . 41

3.11 Rheo-chaos . . . . . . . . . . . . . . . . . . . . . 42

3.12 Slip induced flow instabilities $\ldots \ldots \ldots \ldots$. . . . . . . . . . 43

4.1 Ellipsometry is used to measure changes in polarisation state . . . . . . . 50

$4.2 \quad$ Simulated time variation of intensity in the single scattering regime. . . . . 54

4.3 Small angle light scattering . . . . . . . . . . . . . . . . 56

4.4 Relationship between the free path, mean transport path and size of the scattering centres in the diffusion approximation . . . . . . . . . . . 59

4.5 Multiply scattered light path through some turbid medium . . . . . . . 59

4.6 Simulating the propagator $G_{n}\left(\mathbf{r}_{i}, \mathbf{r}_{o}, l^{*}\right) \ldots \ldots \ldots \ldots$. . . . . . 62

4.7 Simulating the Stokes vector for the diffusion of isotropic and anisotropic

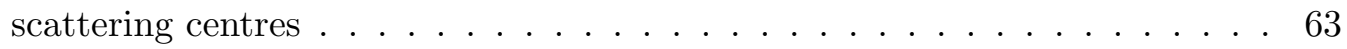

4.8 Static structure factor of a polymer solution . . . . . . . . . . . . . 67

4.9 Dynamic structure factor of an entangled polymer solution . . . . . . . 68

5.1 Strain amplitude sweep $\ldots \ldots \ldots \ldots$. . . . . . . . . . . . . 71

5.2 Linear rheology of the RHB, RHA and RHC samples . . . . . . . . . . . . 72

5.3 Measured linear rheology of RHB, RHA and RHC samples with a comparison to the rheology predicted by the reptation-reaction model . . . . . . . 74

5.4 Nonlinear rheology of the RHB, RHA and RHC samples . . . . . . . . . . 76

5.5 Normal stress differences for the RHB samples $\ldots \ldots \ldots$. . . . . . . . 77

5.6 Nonlinear rheology of selected samples showing transient behaviour. . . . . 79

5.7 Nonlinear rheology of the RHB samples showing transient behaviour . . . . 81

5.8 Dependence of the nonlinear rheology on $\mathrm{CPCl}$ supplier . . . . . . . . . . . 82

5.9 Dependence of the nonlinear rheology on flow geometry . . . . . . . . . 83

5.10 Effects of temperature on the visual appearance of selected samples. . . . . 84

5.11 Temperature dependence of the crystallisation point in the RHB, RHA and RHC wormlike micelles solutions . . . . . . . . . . . . . . . 85

5.12 Visualisation of the $\phi=5.0 \mathrm{wt} . \%$ RHB sample in the cone-plate and parallelplate flow geometries . . . . . . . . . . . . . 87

5.13 Visualisation of the $\phi=8.3$ wt.\% RHB sample in the cone-plate and parallel-plate flow geometries . . . . . . . . . . . . . 88 
5.14 Visualisation of the $\phi=8.3$ wt.\% RHB sample in the cylindrical-Couette

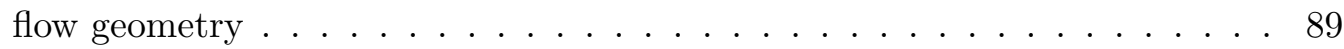

6.1 Experimental set-up for the DWS and PCS microrheology measurements. . 96

6.2 Confirming the timing of the correlator $\ldots \ldots \ldots \ldots$. . . . . . . 96

6.3 DWS and PCS microrheological measurements of RHB samples . . . . . . . 98

6.4 Temperature dependence of the linear rheology of selected RHA and P105

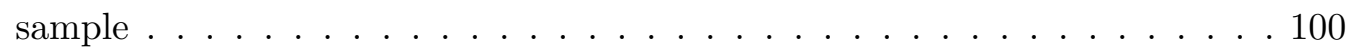

6.5 Dependence of the Poisson-renewal model on adjustable parameters . . . . 102

6.6 Graphical solution to the Poisson-renewal model . . . . . . . . . . . . . . . 103

6.7 Demonstration of the fitting procedure used $\ldots \ldots$. . . . . . . 106

6.8 Fitting the experimental data to the Poisson-renewal model . . . . . . . . 107

6.9 Fitted parameters . . . . . . . . . . . . . . . . . . 108

6.10 Estimate of the average length of the wormlike micelles studied . . . . . . . 109

6.11 SEM micrographs of core-shell particles ～. . . . . . . . . . . . . . 110

6.12 Simulated diffusion NMR measurements, with analysis, for core-shell particles 113

6.13 Mismatch between mechanical rheology and DWS microrheology is observed at low frequencies . . . . . . . . . . . . . . . . . . 114

7.1 Dependence of dephasing on strain rate and mean transport path length in the diffusion approximation . . . . . . . . . . . . . . 116

$7.2 \quad$ Simulation of the propagator $\rho_{n}\left(x_{i}, x, x_{o}, l^{*}\right) \ldots \ldots \ldots \ldots$. . . . . 118

7.3 Predicted flow-DWS correlation for shear thinning fluids undergoing flow . 120

7.4 Experimental set-up for flow-DWS measurements . . . . . . . . . . . . . . 121

7.5 Nonlinear rheology of the samples . . . . . . . . . . . . . . . 122

7.6 Measured flow-DWS correlations for the RHB samples in the cylindricalCouette geometry . . . . . . . . . . . . . . . . . . 124

7.7 $\quad$ Analysis of the flow-DWS correlation measured in the cylindrical-Couette geometry with a comparison of the optical and mechanical flow curves . . . 126

7.8 Bézier spline representation of velocity and strain rate profile with a fit to measured correlations . . . . . . . . . . . . . . 127

7.9 Comparison between strain rate profiles calculated according to the optical flow curve and a fit to the Bézier spline representation strain rate profile . . 128

7.10 Flow-DWS measurements of samples in which the scattering centres are polydisperse . . . . . . . . . . . . . . . . . 129 
7.11 Measured flow-DWS correlation for the PEO and yoghurt samples in the cone-plate geometry . . . . . . . . . . . . . . . . . . 131

7.12 Measured flow-DWS correlation for the RHB samples in the cone-plate

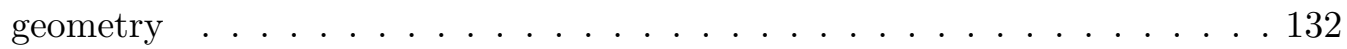

7.13 Analysis of flow-DWS correlations in the cone-plate geometry . . . . . . . . 134

7.14 Measured flow-DWS correlation with analysis of a $\phi=5$ wt. $\%$ and PEO sample in the parallel-plate geometry . . . . . . . . . . . . 136

7.15 Measured flow-DWS correlation with analysis of a $\phi=8$ RHB wormlike

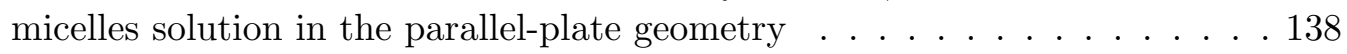

8.1 Experimental set-up for homodyne-PCS/ellipsometry measurements (detail) 143

8.2 Experimental set-up for homodyne-PCS/ellipsometry measurements (overview) 144

8.3 Linear and nonlinear rheology of the RHB samples . . . . . . . . . . . . . . 145

8.4 Measured equilibrium PCS correlations with fit for monodisperse and bidisperse latex sphere suspension, a wormlike micelles solution and PAC solution 147

8.5 Measured equilibrium PCS correlations with fit for the RHA and RHB samples at equilibrium . . . . . . . . . . . . . . 148

8.6 Beam width and transit time velocimetry . . . . . . . . . . . . 150

8.7 Measured PCS correlations with fit, for monodisperse and bidisperse latex sphere suspension under flow . . . . . . . . . . . . . . . . . . . 151

8.8 Measured PCS correlations with fit, for $\phi=3$ and 8 wt.\% RHB samples under flow . . . . . . . . . . . . . . . . . 153

8.9 Fitted local strain rate for selected samples . . . . . . . . . . . . . . . . 153

8.10 Measured homodyne and heterodyne PCS correlations under flow . . . . . . 154

8.11 Apparent velocity of the small angle laser speckle . . . . . . . . . . . . . 155

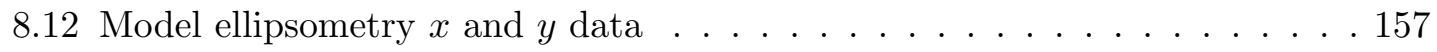

8.13 Determining the correction required for the gold mirrors . . . . . . . . . . 158

8.14 Stray birefringence in the cap and base of the optical geometry . . . . . . . 159

8.15 Point-wise measurements of the local strain rate for $\phi=3-8$ wt.\% RHB wormlike micelles solutions along with a model calculation . . . . . . . . 160

8.16 Point-wise measurements of the scattered intensity for $\phi=3-8 \mathrm{wt} . \%$ RHB wormlike micelles solutions $\ldots \ldots \ldots \ldots$. . . . . . . . . . . . . . . . .

8.17 Point-wise measurements local strain rate and scattered intensity for $\phi=7$ wt.\% RHB wormlike micelles solution . . . . . . . . . . . . . . 163

8.18 Local strain rate measured with a comparison to literature data . . . . . . . 163 
8.19 Excluding multiple scattering from the PCS . . . . . . . . . . . . . 165

8.20 Point-wise measurements of $x$ and $y$ for $\phi=3-8$ wt.\% wormlike micelles . 166

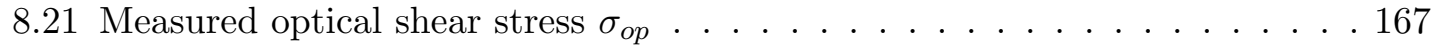

8.22 Positive and negative roots of $x$ and $y \ldots \ldots$. . . . . . . . . 169

8.23 Width of the birefringent band with a comparison to literature data . . . . 169

8.24 Monitoring stress relaxation using ellipsometry . . . . . . . . . . . . . . . 170

8.25 Correlation between strain rate, birefringence and turbidity . . . . . . . . . 171

9.1 Free space geometry and position vectors at the observation plane . . . . . 176

9.2 Model space-time auto and cross-correlation . . . . . . . . . . . . . . . . 177

9.3 Simulated space-time correlation with comparison to model behaviour . . . 179

9.4 Experimental set-up for translation and simple shear flow . . . . . . . . . . 180

9.5 Simulated speckle patterns with increasing path length $d$ of the scattering volume . . . . . . . . . . . . . . . . . . . 181

9.6 Measured and simulated speckle patterns observed with the $d=2$ and 10 mm path length cuvettes . . . . . . . . . . . . . . . . . . 182

9.7 Measured and simulated characteristic times for the translation geometry . 183

9.8 Measured characteristic times for suspensions of latex spheres of different

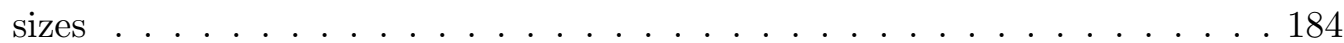

9.9 Measured and simulated speckle patterns observed with the $d=1.5 \mathrm{~mm}$ in the simple shear flow geometry . . . . . . . . . . . . . . 185

9.10 Measured and simulated characteristic times for the simple shear flow ge-

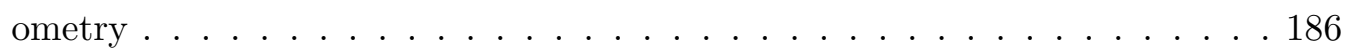

10.1 Flow-concentration coupling? . . . . . . . . . . . . . . . . . . . . . . . 192 


\section{List of Tables}

3.1 Chemical structure of selected surfactant molecules . . . . . . . . . . . . . 28

3.2 Wormlike micelles solutions reported to exhibit shear induced turbidity . . 39

5.1 Composition of the three wormlike micelles systems studied . . . . . . . 70

5.2 Critical rheological parameters $\ldots \ldots \ldots \ldots \ldots$. . . . . . . . 77

6.1 Parameters used in the calculation of data for Poisson-renewal model . . . . 104

6.2 Comparison of the elastic modulus $G_{e}$ with estimates $G_{e x t}$ and $2 G_{L O}$. . . . 104

8.1 Stress-optic coefficient reported for wormlike micelles solutions in the liter-

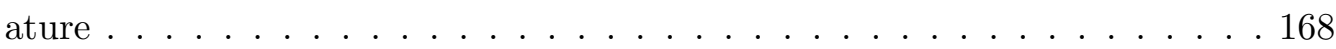




\section{Chapter 1}

\section{Thesis overview}

In appropriate conditions soap-like surfactant molecules in solution can assemble into long microscopic wormlike structures called wormlike micelles. The resulting wormlike micelles solution exhibits both solid-like and liquid-like characteristics and is known as a complex fluid. These solutions are sometimes observed to flow in a peculiar way that suggests that the viscosity of the fluid varies discontinuously. This discontinuous banding has generated considerable interest because of its general implications for the flow behaviour of complex fluids.

Wormlike micelles solutions have been actively studied for over 20 years with well over 1000 papers published on the subject between 1990 and 2009. 17 of these papers describe research conducted in the Author's own laboratory using rheo-nuclear magnetic resonance (rheo-NMR) methods. The work described in this thesis, however, focuses almost exclusively on using rheo-optical methods in conjunction with conventional rheomechanical techniques to understand the physical properties of wormlike micelles solutions and is intended to complement the group's previous rheo-NMR work.

This thesis is divided into ten chapters. Chapters 2-4 review rheology (Chapter 2), the rheology of wormlike micelles (Chapter 3) and rheo-optics (Chapter 4). Chapters 5-9 describe original rheo-mechanical and rheo-optic work using visualisation (Chapter 5), diffusing wave spectroscopy (Chapters 6-7), ellipsometry (Chapter 8) and photo-correlation spectroscopy (Chapters 8-9). The thesis concludes with Chapter 10.

Rheology is the study of flow and deformation. In Chapter 2 the subject of rheology is formally introduced. Rheology is conventionally studied using a mechanical spectroscopy called rheometry in either a small deformation linear regime or a large deformation nonlinear or flow regime. The rheology of complex fluids depends on the interaction of the constitutive units, which are often small enough to be influenced by thermal or Brownian forces. Because understanding this influence can be important to understanding the bulk rheology, the Brownian motion of microscopic particles and its relationship to the mean square displacement (MSD) is considered here in several environments. The chapter concludes with a discussion of the model rheology of polymer systems, including a descrip- 
tion of the reptation model, which asserts that the rheology of some polymer systems is determined in part by snake-like curvilinear thermal diffusion of the polymer strands.

Wormlike micelles are a polymer-like self-assembly of surfactant molecules that can form in solution in the appropriate conditions. Unlike polymers where the length is fixed at polymerisation, wormlike micelles are formed dynamically through a continuous process of breakage and recombination. Wormlike micelles solutions possess several unusual rheological properties and have been actively studied for many years. In Chapter 3 the rheology of wormlike micelles solutions is reviewed. The reptation-reaction model, which adapts the reptation model to include the effects of breakage and recombination and is a widely accepted description of the low frequency linear rheology, is introduced and experimental evidence for the later Poisson-renewal model, which extends the description of the reptation-reaction model to include high frequency modes, is considered and questioned. Implications of the reptation-reaction model for the non-linear rheology including the important prediction that in appropriate conditions a wormlike micelles solution would spatially partition into strain rate bands or 'shear bands' in constant strain rate or 'simple shear flow' conditions is discussed. Indirect evidence from flow-birefringence studies and direct evidence from velocimetry studies is presented verifying that wormlike micelles solutions do in fact shear band. Other phenomenology associated with the shear flow of wormlike micelles solutions including possible coupling between flow and concentration and the presence of stress fluctuation and flow instabilities, or rheo-chaos, are also discussed along with the predictions of several continuum models.

A mechanical rheometer reports spatially averaged quantities. This spatial averaging can obscure the true flow behaviour of complex fluids that undergo some kind of spatial partitioning like that seen in wormlike micelles solutions. Furthermore, a mechanical rheometer is subject to mechanical limitations that restrict its operating range. To address these issues the rheology of a complex fluid can be studied using other experimental techniques. This thesis focuses on rheo-optical methods. In Chapter 4 rheo-optics is reviewed with an emphasis on those optical techniques used to study wormlike micelles solutions here. Plane wave and Green function solutions to Maxwell equations are introduced and are used to understand how the physical properties of transmitted or scattered light can be related to the optical properties of the medium. The optical technique ellipsometry, which is often called flow birefringence in the context of rheology, and the dynamic light scattering techniques: homodyne photo-correlation spectroscopy (PCS) and diffusing wave spectroscopy (DWS) are introduced. The chapter concludes with a discussion of selected rheo-optical properties of model polymer systems, which will subsequently be used to understand some of the rheo-optical properties of wormlike micelles solutions.

The experimental component of the thesis begins with a study of the rheology of three wormlike micelles solution systems (RHB, RHA and RHC) based on the surfactant cetylpyridinium chloride in water using mechanical rheometry in Chapter 5. Both the linear and nonlinear rheometry of these systems show reasonable agreement to the predictions of the reptation-reaction model. While significant stress fluctuations were noted in one particular flow geometry, they were generally not observed in most rheo-mechanical 
measurements presented here, indicating that these fluctuations are not universal and that they are geometry dependent. In addition to studying the samples using rheo-mechanical methods, several samples are studied using the simplest rheo-optic technique: direct visualisation. Here, pronounced turbidity is seen in the RHB samples with turbid rings forming in samples expected to shear band in several flow geometries.

The reptation-reaction model examined in Chapter 4 describes the low frequency linear rheology of wormlike micelles solutions. The Poisson-renewal model extends the reptation-reaction model to include the effect of high frequency modes. This model can potentially be used to estimate the average length of the wormlike micelles in solution - a quantity that is difficult to determine using more conventional techniques. The Poissonrenewal model has been difficult to validate experimentally because of the upper frequency limit imposed by inertia in conventional mechanical rheometry. In recent years, a new technique called microrheology where the linear rheology is inferred from the Brownian motion of embedded microscopic probe particles has been developed. The frequency response of the technique exceeds that of mechanical rheometry by 3-4 decades making it a potentially useful method for testing the Poisson-renewal model. In Chapter 6 DWS microrheology is used to measure the linear rheology of selected RHB samples over an extended frequency range. These measurements along with rheo-mechanical measurements and literature data are compared to the predictions of the Poisson-renewal model by fitting the data. Qualitatively, the fit does match much of the experimental data (with some exceptions) and gives physically reasonable values of the average micelles length (with some reservations). In principle, any technique that can be used to determine the MSD of embedded microscopic probe particles undergoing Brownian motion can be used for microrheology; the potential of diffusion NMR as a microrheological technique is also considered in this chapter.

While DWS has been successfully applied to the study of the linear rheology of complex fluids, the technique's capacity to characterise the nonlinear rheology or flow behaviour of complex fluids is largely unexplored. This is addressed in Chapter 7 where 'flow-DWS' is used to study the unusual flow behaviour predicted in Chapter 3 to occur in some wormlike micelles solutions. Here, DWS's spatial sensitivity to shear induced relative motion is used to infer the flow behaviour in the three standard rotational flow geometries used in conventional mechanical rheometry: cylindrical-Couette, cone-plate and parallelplate geometries. In the cylindrical-Couette geometry, the flow-DWS measurements are largely consistent with predictions based on rheo-mechanical measurements and indicate that some RHB wormlike micelles solutions are partitioning into quite stable strain rate bands. Flow-DWS measurements in the cone-plate and parallel-plate geometries were more difficult to interpret, suggesting that the flow in these geometries is more complicated than that observed in the cylindrical-Couette geometry.

Shear banding in wormlike micelles was first demonstrated indirectly using flow birefringence, a rheo-optic technique that probes optical anisotropy. Here, birefringence bands were observed in some sheared wormlike micelles solutions suggesting that partitioning into strain rate bands was occurring. Partitioning into strain rate bands has been since observed directly using velocimetry techniques. Rheo-NMR is unusual amongst the 
techniques commonly coupled to rheology in that it is capable of simultaneously resolving both anisotropy and velocity. Measurements of a sheared wormlike micelles solution using rheo-NMR led workers in the Author's own lab to question the assumption that birefringence and strain rate bands necessarily coincided. This cast some doubt on the interpretation of the flow birefringence measurements. To investigate this relationship more directly, an approach combining homodyne PCS and ellipsometry to measure the strain rate and optical anisotropy simultaneously is used to examine a series of RHB wormlike micelles solutions in a cylindrical-Couette geometry in Chapter 8. Shear banding is observed in some samples and these shear bands are observed to coincide with birefringence bands. Furthermore, it is noted that the high strain rate band was significantly more turbid than the low strain rate band suggesting a connection between shear rate, optical anisotropy and turbidity.

In Chapter 8 a rather curious observation is made: the dynamic laser speckle pattern produced by scattering centres embedded in a flowing fluid appears to circulate slowly about the unscattered transmitted beam. In Chapter 9 this phenomenon is studied quantitatively with a view to developing a homodyne PCS/ellipsometry based experimental technique capable of resolving both flow and anisotropy independently at small angles using a camera based detection system. To understand the circulation described above, the dynamic speckle pattern is simulated by considering the field radiated from a random ensemble of scattering centres moving through a laser, and its statistical behaviour is summarised using space-time correlation. These simulated space-time statistics are quantitatively comparable to the space-time statistics measured in two simple flow configurations in a number of optical set-ups. Unfortunately, due to issues with a homemade cylindrical-Couette geometry, it was not possible to test the proposed homodyne PCS/ellipsometry technique experimentally.

The thesis concludes in Chapter 10 with a summary and introduces a speculative model that relates the turbidity observed in the high strain rate band to the formation of metastable structures that temporarily sequester surfactant from solution, reducing the local concentration of surfactant, length or degree of entanglement of the wormlike micelles and viscosity. 


\section{Chapter 2}

\section{Rheology}

\section{$2.1 \quad$ Introduction}

Soft condensed matter (European) or complex fluids (US) are a broad class of materials that possess mechanical properties that fall between those of classical solids and liquids [1. A general feature of these complex fluid systems is the presence of structures on nanoscopic to microscopic length scales. It is the interaction and reorganisation of these structures that give rise to these fluids' 'complex' mechanical properties. Because the energies of interaction are typically of the order $k_{B} T$ these materials appear 'soft' at room temperatures.

The study of the relationship between the deformation and flow and the microstructure of complex fluids is called rheology. In this chapter the subject of rheology is formally introduced. This introduction is followed by a discussion of the influence of thermal agitation on small particles. Finally, because of their historical significance and similarity to the system studied here, the rheology of polymer-like systems is discussed.
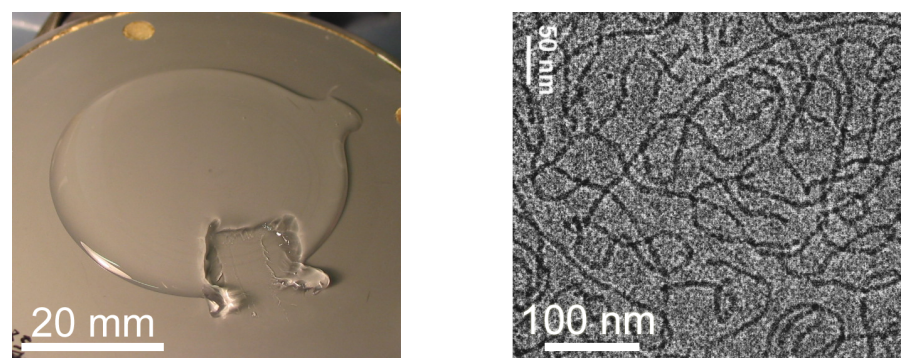

Figure 2.1: The macroscopic and microscopic appearance of a wormlike micelles solution - the complex fluid studied here. Micrograph from [2]. 


\section{$2.2 \quad$ Rheology}

\subsubsection{Flow, stress and the constitutive relation}

The flow behavior of a fluid can be determined by solving the force balance equation $(f=m a)$ which gives the time rate of change of momentum $\rho \mathbf{v}$ in terms of the momentum flux [3]

$$
\frac{d}{d t} \int \rho \mathbf{v} d V=\underbrace{\int_{S} \rho \mathbf{v}[\hat{\mathbf{n}} \cdot \mathbf{v}] d S}_{\text {convection }}+\underbrace{\int_{V} \rho \mathbf{g} d V}_{\text {external forces }}+\underbrace{\int_{S}[\hat{\mathbf{n}} \cdot \underline{\underline{T}}] d S}_{\text {internal forces }} .
$$

The first flux term on the RHS is associated with the convection of momentum through a closed surface $S$, with surface normal $\hat{\mathbf{n}}$. The second term describes the contribution of external forces over a volume $V$; here it is assumed that only gravity $\mathrm{g}$ affects the momentum flux. The final term is associated with internal molecular forces. The total stress tensor $\underline{\underline{T}}$ describes the force per unit area on the surface area of a differential volume element due to the relative motion of surrounding molecules. This tensor is generally symmetric and can be decomposed into a component associated with a thermodynamic pressure $p$ and a component that will be associated with flow [3]

$$
\underline{\underline{T}}=\underline{\underline{\sigma}}+p \underline{\underline{I}}
$$

Using Gauss's law Eqn. 2.1 can be expressed in differential form

$$
\frac{\partial[\rho \mathbf{v}]}{\partial t}=-\nabla \cdot[\rho \mathbf{v v}]-\nabla \cdot \underline{\underline{T}}+\rho \mathbf{g}
$$

In order to solve differential Eqn. 2.3 a constitutive relation between stress and velocity must be assumed. With this relation and boundary conditions the fluid's flow behaviour can (in principle) be determined. This constitutive relation between stress and flow is studied in rheometry. Eqn. 2.3 is not straightforward to solve for an arbitrary experimental geometry; in rheometry only very simple flow geometries are used.

\subsubsection{Simple shear flow}

In the simple shear or planar-Couette flow geometry, the material of interest is trapped between two infinite parallel surfaces separated by a gap $d$ that undergo relative transla-

tion. For the planar-Couette geometry illustrated in Fig. 2.2 the stress tensor takes the form

$$
\underline{\underline{\sigma}}=\left[\begin{array}{ccc}
\sigma_{11} & \sigma & 0 \\
\sigma & \sigma_{22} & 0 \\
0 & 0 & \sigma_{33}
\end{array}\right]
$$




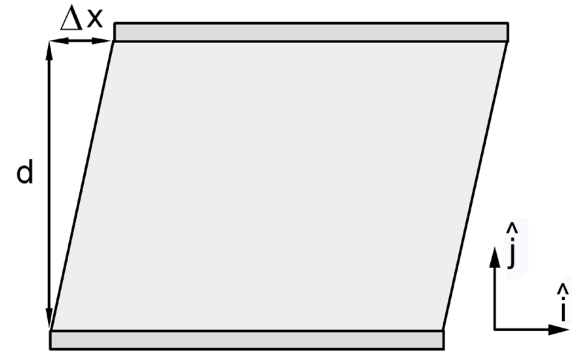

Figure 2.2: Simple shear or planar-Couette flow geometry.

with $\sigma_{12}=\sigma_{21}=\sigma$ because of symmetry. This relation can be deduced from the force balance equation [4]. $\sigma$ is called the shear stress and $\sigma_{11}, \sigma_{22}$ and $\sigma_{33}$ the normal stresses. Normal stresses are not accessible experimentally; however, the normal stress differences:

$$
\begin{aligned}
& N_{1}=\sigma_{11}-\sigma_{22}, \\
& N_{2}=\sigma_{22}-\sigma_{33},
\end{aligned}
$$

may be.

The response of material to a stress depends on the constitutive relation. The constitutive relation for a Hookean solid (a solid that obeys Hooke's law) is

$$
\underline{\underline{\sigma}}=G \underline{\underline{C}}^{-1}
$$

where $G$ is the elastic modulus of the solid material and $\underline{\underline{C}}^{-1}$ is called the finger tensor defined as $\underline{\underline{C}}^{-1}=\left(\underline{\underline{F}}^{-1}\right)^{T} \underline{\underline{F}}^{-1}$ where $\underline{\underline{F}}^{-1}=\frac{\partial \mathbf{r}^{\prime}}{\partial \mathbf{r}}$ is the deformation gradient tensor. For the simple shear flow the 'deformation' accumulated between times $t$ and $t^{\prime}$ is [5]

$$
\underline{\underline{F}}^{-1}\left(t, t^{\prime}\right)=\frac{\partial \mathbf{r}^{\prime}}{\partial \mathbf{r}}=\left[\begin{array}{lll}
\frac{\partial r_{1}^{\prime}}{\partial r_{\gamma}} & \frac{\partial r_{2}^{\prime}}{\partial r_{\gamma}} & \frac{\partial r_{3}^{\prime}}{\partial r_{\gamma}} \\
\frac{\partial r_{1}}{\partial r_{2}} & \frac{\partial r_{2}^{\prime}}{\partial r_{2}} & \frac{\partial r_{3}}{\partial r_{2}} \\
\frac{\partial r_{1}^{\prime}}{\partial r_{3}} & \frac{\partial r_{2}^{\prime}}{\partial r_{3}} & \frac{\partial r_{3}^{\prime}}{\partial r_{1}}
\end{array}\right]=\left[\begin{array}{lll}
1 & 0 & 0 \\
\gamma & 1 & 0 \\
0 & 0 & 1
\end{array}\right] .
$$

$\gamma=\Delta x / d$ is called the strain. Eqn. 2.7 implies that

$$
\sigma=G \gamma
$$

which is Hooke's Law.

The constitutive relation for a Newtonian fluid satisfies the relation

$$
\underline{\underline{\sigma}}=2 \eta \underline{\underline{D}},
$$


where $\eta$ is the viscosity of the fluid. $\underline{\underline{D}}$ is called the rate of deformation tensor and is defined as $2 \underline{\underline{D}} \equiv(\nabla \mathbf{v})^{T}+\nabla \mathbf{v}$ where $\nabla \mathbf{v}=\frac{\partial \mathbf{v}}{\partial \mathbf{r}}$ is the gradient velocity tensor. For the simple shear flow [5]

$$
\nabla \mathbf{v}=\frac{\partial \mathbf{v}}{\partial \mathbf{r}}=\left[\begin{array}{lll}
\frac{\partial v_{1}}{\partial r_{1}} & \frac{\partial v_{2}}{\partial r_{1}} & \frac{\partial v_{3}}{\partial r_{1}} \\
\frac{\partial v_{1}}{\partial r_{2}} & \frac{\partial v_{2}}{\partial r_{2}} & \frac{\partial v_{3}}{\partial r_{2}} \\
\frac{\partial v_{1}}{\partial r_{3}} & \frac{\partial v_{2}}{\partial r_{3}} & \frac{\partial v_{3}}{\partial r_{1}}
\end{array}\right]=\left[\begin{array}{ccc}
0 & 0 & 0 \\
\dot{\gamma} & 0 & 0 \\
0 & 0 & 0
\end{array}\right]
$$

$\dot{\gamma}=\partial v_{x} / \partial y=\partial \gamma / \partial t$ is called the strain rate. Eqn. 2.10 implies that

$$
\sigma=\eta \dot{\gamma}
$$

which is Newton's law for fluids. If a fluid is Newtonian Eqn. 2.3 can be reformulated as the Navier-Stokes equation [6]

$$
\rho\left[\frac{\partial \mathbf{v}}{\partial t}+\mathbf{v} \cdot \nabla \mathbf{v}\right]=-\nabla p+\eta \nabla^{2} \mathbf{v}+\rho \mathbf{g}
$$

( $\rho$ constant - incompressible fluid) which is commonly solved in fluid mechanics problems.

Both Newtonian fluids and Hookean solids are free of hysteresis or 'memory'. Hysteresis effects are common and significantly complicate the description of the constitutive relation. Following Larson [5], consider first a material with a constitutive relation that satisfies the differential equation

$$
\sigma+\tau \frac{\partial \sigma}{\partial t}=G \tau \dot{\gamma}
$$

or equivalently in integral form

$$
\begin{aligned}
\sigma(t) & =\int_{-\infty}^{t} G \exp \left[\left(t^{\prime}-t\right) / \tau\right] \dot{\gamma}\left(t^{\prime}\right) d t^{\prime} \\
& =\int_{-\infty}^{t} \frac{G}{\tau} \exp \left[\left(t^{\prime}-t\right) / \tau\right] \gamma\left(t, t^{\prime}\right) d t^{\prime}
\end{aligned}
$$

where $\tau$ is a characteristic relaxation time and $\gamma\left(t, t^{\prime}\right)$ is the strain accumulated between the times $t$ and $t^{\prime}$. This material is called a Maxwell fluid. As is indicated by Eqn. 2.16 the current stress depends on the strain history of the material [7]. Equations of the form 2.14 2.16 occur in mechanical (and electrical) circuits. A mechanical circuit analogue for a Maxwell fluid is shown in Fig. 2.3 i. It is composed of a spring (of spring constant $G$ ) coupled in series to a dashpot (of viscosity $\eta$ ) subject to a driving force $f$ (an analogue of $\sigma$ ) which produces a displacement $x$ (an analogue of $\gamma$ ). For times $t-t^{\prime}<<\tau$ the behaviour of the fluid is dominated by the shear modulus (the spring in the analogue) and $\sigma=G \gamma$ whereas at $t-t^{\prime}>>\tau$ the behaviour is dominated by the viscous contribution (dashpot) and $\sigma=G \tau \dot{\gamma}=\eta \dot{\gamma}$. Such a fluid is said to be viscoelastic - the mechanical behaviour interpolates between that of viscous (Newtonian) fluids and elastic (Hookean) 

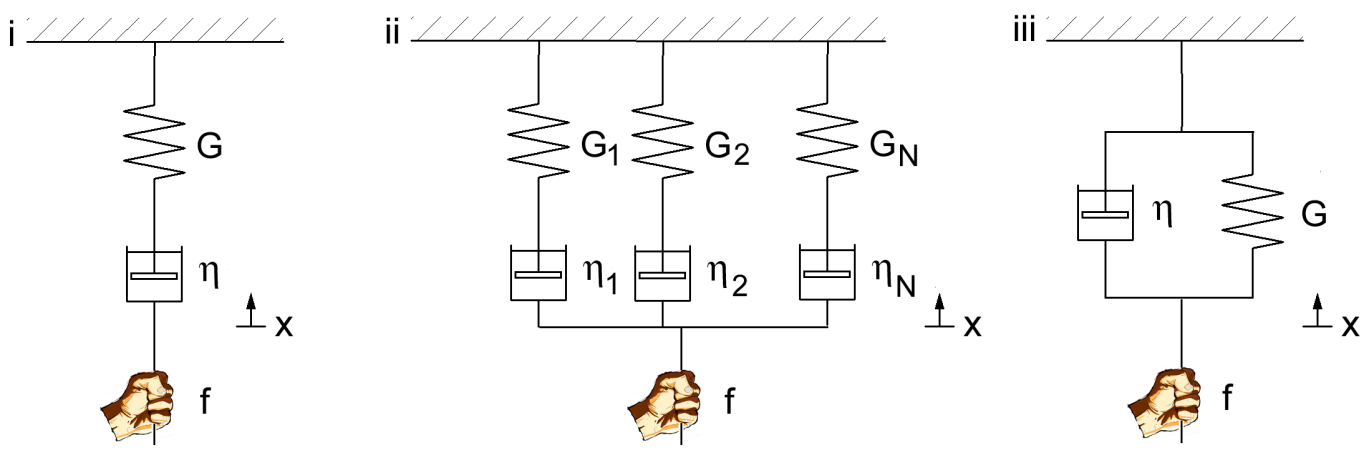

Figure 2.3: Spring-dashpot mechanical circuit analogues used in rheology. i) A single Maxwell element, ii) a system of Maxwell elements and iii) an alternative arrangement called a Kelvin element.

solids. As is suggested by Fig. 2.3 ii additional Maxwell elements can be added to the circuit leading to a shear stress

$$
\sigma(t)=\int_{-\infty}^{t} \sum_{i} G_{i} \exp \left[\left(t^{\prime}-t\right) / \tau_{i}\right] \dot{\gamma}\left(t^{\prime}\right) d t^{\prime} .
$$

For a continuous distribution of Maxwell elements such that $\sum_{i} G_{i} \exp \left[-t / \tau_{i}\right] \rightarrow \int G_{0}(\tau) \exp [-t / \tau] d \tau$ the stress is

$$
\begin{aligned}
\sigma(t) & =\int_{-\infty}^{t} G\left(t^{\prime}-t\right) \dot{\gamma}\left(t^{\prime}\right) d t^{\prime} \\
& =\int_{-\infty}^{t} m\left(t^{\prime}-t\right) \gamma\left(t^{\prime}, t\right) d t^{\prime} .
\end{aligned}
$$

$G\left(t^{\prime}-t\right)=\int G_{0}(\tau) \exp \left[\left(t^{\prime}-t\right) / \tau\right] d \tau$ is called the stress relaxation modulus and $m\left(t^{\prime}-t\right)$ is called a memory function and is related to the stress relaxation modulus by [7]

$$
m\left(t-t^{\prime}\right)=\frac{\partial G\left(t^{\prime}-t\right)}{\partial t^{\prime}}
$$

More generally the stress - accumulated strain relation can be expressed as a tensor (with $\sigma \rightarrow \underline{\underline{\sigma}}(t)$ and $\left.\gamma\left(t^{\prime}, t\right) \rightarrow \underline{\underline{C}}\left(t^{\prime}, t\right)\right)[5]$

$$
\underline{\underline{\sigma}}(t)=\int_{-\infty}^{t} m\left(t^{\prime}-t\right) \underline{\underline{C}}^{-1}\left(t^{\prime}, t\right) d t
$$

Eqn. 2.21 says that the stress 'now' depends on the deformation history of the sample and the sample's 'memory' of this deformation history. Other configurations of springs and dashpots are sometimes used to describe viscoelastic properties of complex fluids. In Fig. 2.3 iii a spring and dashpot are coupled in parallel - this configuration is called a Kelvin element. 


\subsubsection{Linear rheology}

Linear rheology is restricted to the study of complex fluids in the small strain limit where the output stress is proportional to the input strain. In this linear regime the stress relaxation function (Eqn. 2.18) can be measured in the time domain by applying a step strain at $t^{\prime}=0$ of amplitude $\gamma=\gamma_{0}$ so that $\dot{\gamma}\left(t^{\prime}\right)=\gamma_{0} \delta(0)$ and considering the ratio of stress to deformation

$$
G(t)=\frac{\sigma(t)}{\gamma_{0}}
$$

More commonly the complex shear modulus $G^{*}(\omega)$ is measured in the frequency domain by applying a sinusoidal strain $\gamma=\gamma_{0} \sin \omega t$ and measuring the complex amplitude of the resulting oscillating stress. This ratio of stress to strain can be resolved into a real component in phase with the input deformation called the storage modulus $\left(G^{\prime}\right)$ and an imaginary component out of phase with input called the loss modulus $\left(G^{\prime \prime}\right)$ where

$$
G^{*}(\omega)=\frac{\sigma^{\prime}(\omega)+i \sigma^{\prime \prime}(\omega)}{\gamma_{0}}=G^{\prime}(\omega)+i G^{\prime \prime}(\omega) .
$$

The complex shear modulus is related to the stress relaxation modulus by the integral transform

$$
G^{*}(\omega)=i \omega \int_{0}^{\infty} G(t) \exp [i \omega t] d t .
$$

Alternatively the linear rheology can be described in terms of the complex shear viscosity $\eta^{*}(\omega)$

$$
G^{*}(\omega)=i \omega \eta^{*}(i \omega)
$$

The complex shear viscosity represents the mechanical impedance of a complex fluid [7].

\subsubsection{Nonlinear rheology}

Nonlinear rheology is the study of the constitutive relation in the large strain limit where the output stress is no longer proportional to the input strain. It is most commonly studied by applying a constant strain rate to the material and measuring the steady-state stress

after all transient behaviour has died away. Typically, soft materials show a strain rate dependent viscosity

$$
\sigma=\eta(\dot{\gamma}) \dot{\gamma}
$$

Often power law dependence of the form $\eta(\dot{\gamma}) \propto \dot{\gamma}^{p}$ is observed. Materials can be classified based on the exponent $p$. The material is said to be Newtonian if $p=0$, shear thinning if $p<0$, and shear thickening if $p>0$ [7]. The relationship between viscosity and strain rate and stress and strain rate for the three types of fluids is illustrated in 2.4. The stress versus strain rate representation is called a flow curve. 

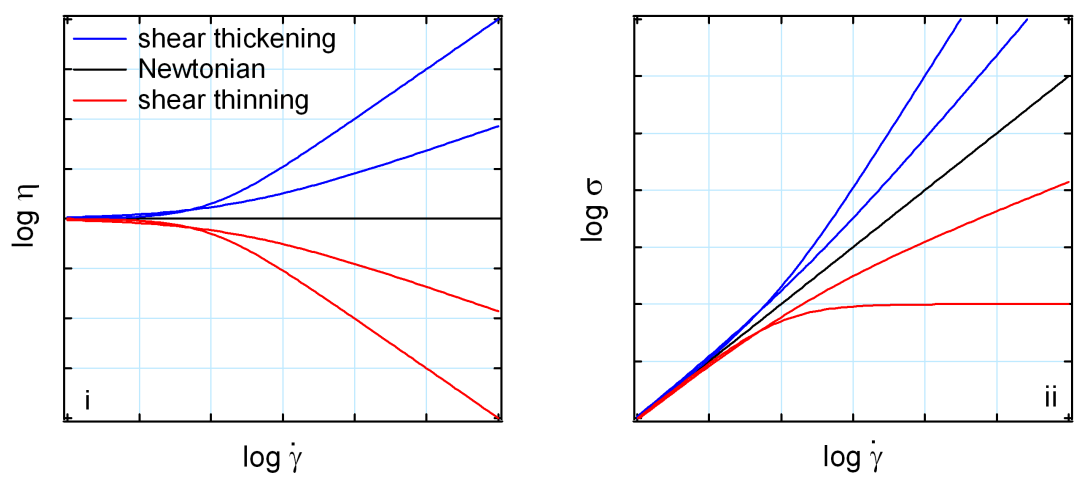

Figure 2.4: Two representations of the nonlinear steady-state flow behaviour of shear thinning, Newtonian and shear thickening fluids. Note the log-log axes.

\subsubsection{Mechanical rheometry}

A mechanical rheometer is a tool used to probe the constitutive relation. In a rheometer, a shear stress or strain is directly applied to the sample and the resulting strain or stress is measured. Unfortunately, simple shear (or planar-Couette) flow cannot be realised experimentally. In practice, several geometries which approximate simple shear flow are used. They are cylindrical-Couette, cone-plate and parallel-plate geometries.

In the cylindrical-Couette geometry, the fluid is trapped between two coaxial cylinders of radii $r_{i}$ and $r_{o}$. Either the inner or outer cylinder rotates. Ignoring end effects, the shear stress (in cylindrical coordinates) in this geometry is given by

$$
\sigma_{\phi, r}(r)=\sigma_{0}\left[\frac{r_{i}}{r}\right]^{2}
$$

where $\sigma_{0}$ is the shear stress at the inner wall. In contrast to the planar-Couette geometry where the shear stress is constant within the fluid, in the cylindrical-Couette geometry the shear stress decreases from the inner wall. In order to minimise the stress variation across the gap, the gap between the two cylinders is deliberately made small. In terms of the angular velocity $(\omega)$ of rotating cylinder and torque $(T)$ required to sustain this rotation, the measured or 'apparent' stress and strain rates are [3]:

$$
\begin{aligned}
\sigma & =\frac{T}{2 \pi l r_{i}^{2}}, \\
\dot{\gamma} & =\frac{r_{i} \omega}{r_{o}-r_{i}} .
\end{aligned}
$$

In the cone-plate geometry, the fluid is trapped between the tip of a cone in contact with a flat plate at a right angle to the cone's axis of symmetry. The cone or plate rotates about this axis. Ignoring edge effects, the shear stress is given (in polar coordinates) by

$$
\sigma_{\phi, \theta}(\theta)=\sigma_{0} \operatorname{cosec}^{2}[\pi / 2-\theta],
$$



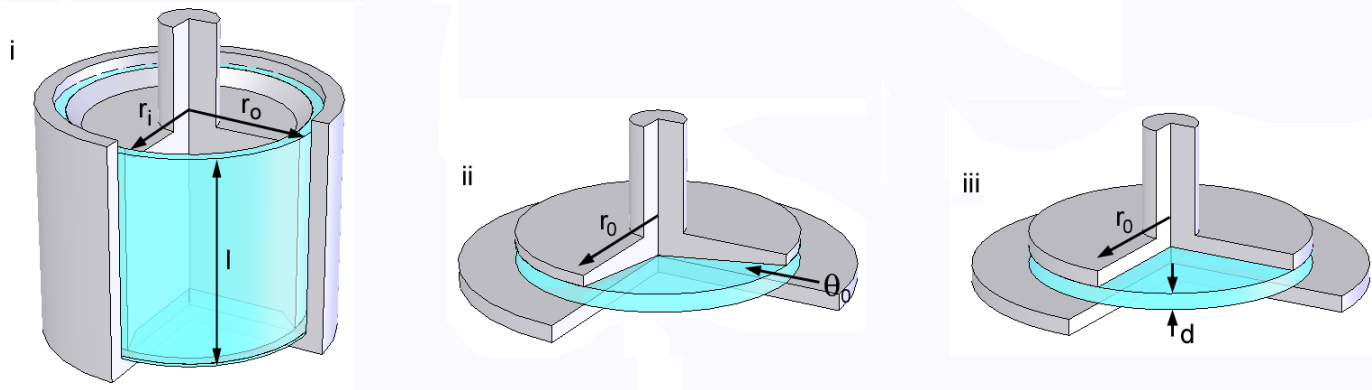

Figure 2.5: Standard flow geometries used in rheometry. i) Cylindrical-Couette, ii) coneplate and iii) parallel-plate geometries.

where $\sigma_{0}$ is the wall stress at the plate. As with the cylindrical-Couette geometry, the stress is not uniform within the gap; however, for the cone angles typically used (1-4 deg) it is significantly more uniform than that observed in the cylindrical-Couette geometry. It is mechanical rheometry's closest approximation to planar-Couette flow. In terms of the angular velocity, torque and thrust $f$ on the cone, the measured stress, strain rate and first normal stress difference is [3]:

$$
\begin{aligned}
\sigma & =\frac{3 T}{2 \pi r_{0}^{3}}, \\
\dot{\gamma} & =\omega / \theta_{0}, \\
N_{1} & =\frac{2 f}{\pi r_{0}^{2}},
\end{aligned}
$$

where small values of the cone angle $\theta_{0}$ have been assumed.

In the parallel-plate geometry, the fluid is trapped between a disc and a parallelplate with a disc-plate separation $d$. The disc rotates. In this geometry, the shear stress is uniform along an axis parallel to the axis of rotation; however it will vary radially. Because the flow is locally planar-Couette (from the force balance equations [4]) the local strain rate (assuming that the complex fluid is well behaved) varies radially

$$
\dot{\gamma}(r)=\omega r / d
$$

The measured torque is an integral over the radially varying wall stress

$$
T=2 \pi \int_{0}^{r_{0}} \sigma_{0}(r) r^{2} d r
$$

This integral over unknown $\sigma_{0}(r)$ complicates the relationship between stress and torque. Because of this complication, this geometry is not commonly used for nonlinear rheology. 
In instances where the geometry is used here, the stress and strain rate is reported as [3]:

$$
\begin{aligned}
\sigma & =\frac{2 T}{\pi r_{0}^{3}}, \\
\dot{\gamma} & =\frac{\omega r_{0}}{d} .
\end{aligned}
$$

\subsection{Brownian motion and diffusion}

The interaction and rearrangement of the microscopic structural units of a complex fluid are often influenced by random thermal or Brownian forces which induce Brownian motion and diffusion. The relationship between these forces and the resulting motion can be understood statistically through the fluctuation-dissipation theorem [8]. Continuing with the mechanical analogy developed in the last section, the impedance of a mechanical circuit is defined as 9

$$
Z(\omega) \equiv F(\omega) / V(\omega)
$$

where $F(\omega)$ is a force and $V(\omega)$ is the resulting velocity. The fluctuation-dissipation theorem relates the power spectral density of a thermal driving force to the impedance of the mechanical circuit [10]

$$
F(\omega) F^{*}(\omega)=4 k_{B} T \Re e[Z(\omega)] .
$$

The dynamic behavior of an element in a mechanical circuit due to random thermal forces can be determined by combining Eqn. 2.38 and 2.39 and its statistics summarised by a mean square displacement (MSD). The MSD is defined by the velocity autocorrelation function [1]

$$
\left\langle\Delta r^{2}(t)\right\rangle \equiv 2 \int_{0}^{t}(t-\tau)\langle v(0) v(\tau)\rangle d \tau .
$$

Noting that velocity autocorrelation and velocity power spectrum are Fourier Transform pairs, $\left\langle v(0) v^{*}(\tau)\right\rangle=\int_{-\infty}^{\infty} V(\omega) V^{*}(\omega) e^{i \omega \tau} d \omega$, the MSD is

$$
\begin{aligned}
\left\langle\Delta r^{2}(t)\right\rangle & =2 \int_{0}^{t}(t-\tau) \frac{1}{2 \pi}\left[\int_{-\infty}^{\infty} V(\omega) V^{*}(\omega) e^{i \omega \tau} d \omega\right] d \tau \\
& =\frac{1}{\pi} \int_{0}^{t}(t-\tau)\left[\int_{-\infty}^{\infty} \frac{4 k_{B} T \Re e[Z(\omega)]}{Z(\omega) Z^{*}(\omega)} e^{i \omega \tau} d \omega\right] d \tau
\end{aligned}
$$

Eqn. 2.42 relates average motion to thermal agitation. To illustrate, consider a Kelvin element (Fig. 2.3 iii) coupled with a mass $m$ in series driven by a force $f(t)$. The equation of motion of the mass is

$$
m \ddot{x}(t)+\zeta \dot{x}(t)+k x(t)=f(t)
$$


( $\zeta=\eta$ in this mechanical analogue). This impedance of the circuit can be determined by assuming that the forcing term is sinusoidal $f(t)=F(\omega) e^{i \omega t}$ and that the resulting velocity is also sinusoidal $v(t)=V(\omega) e^{i \omega t}$. This gives an impedance $Z(\omega)=\frac{k}{i \omega}+\zeta+i m \omega$ and an MSD

$$
\left\langle\Delta r^{2}(t)\right\rangle=\frac{1}{\pi} \int_{0}^{t}(t-\tau)\left[\int_{-\infty}^{+\infty} \frac{4 k_{B} T \eta \omega^{2}}{\left(k-m \omega^{2}\right)^{2}+\eta^{2} \omega^{2}} e^{i \omega \tau} d \omega\right] d \tau .
$$

Einstein asserted that Brownian motion of the small neutrally buoyant sphere of mass $m$ under influence of random thermal forces $f(t)$ and viscous drag $\zeta \dot{x}$ was described by Eqn. 2.43 with $k=0$. When $k=0, m \neq 0$ and $\eta \neq 0$ Eqn. 2.44 simplifies to

$$
\left\langle\Delta r^{2}(t)\right\rangle=4 k_{B} T\left[\frac{t}{\eta}-\frac{m}{\eta^{2}}[1-\exp (-t \zeta / m)]\right]=2\left[2 D t-2 D \frac{m}{\zeta}\left(1-\exp \left[\frac{-t \zeta}{m}\right]\right)\right],
$$

where

$$
D=\frac{k_{B} T}{\zeta}
$$

$D$ is called the diffusion constant. Assuming that the viscous drag force on the sphere follows Stokes law $\zeta=6 \pi \eta a$ [1], where $\eta$ is the viscosity of the Newtonian host and $a$ is the sphere radius, the diffusion constant is

$$
D=\frac{k_{B} T}{6 \pi \eta a}
$$

This is the Stokes-Einstein relation [11. Eqn. 2.45 describes the MSD in one dimension; in three dimensions the MSD is a factor of three larger. At short times the MSD is a quadratic function of time. This is called the ballistic regime. For $t \gg \zeta / m$ the second term is negligible and the three-dimensional MSD is

$$
\left\langle\Delta r^{2}(t)\right\rangle \approx 6 D t
$$

If the ballistic regime can be neglected, the probable space-time behaviour of the isolated Brownian particle can be determined from the diffusion equation

$$
\frac{\partial G(\mathbf{r}, t)}{\partial t}=D \nabla^{2} G(\mathbf{r}, t)
$$

$G(\mathbf{r}, t)$ is a propagator which gives probability that the particle, initially at the origin at $t=0$, will be at $\mathbf{r}$ at time $t$. For free diffusion $G(\mathbf{r}, t)$ is a Gaussian function which spreads in time and space [1]

$$
G(\mathbf{r}, t)=(4 \pi D t)^{-3 / 2} \exp \left[-\frac{r^{2}}{4 D t}\right]
$$



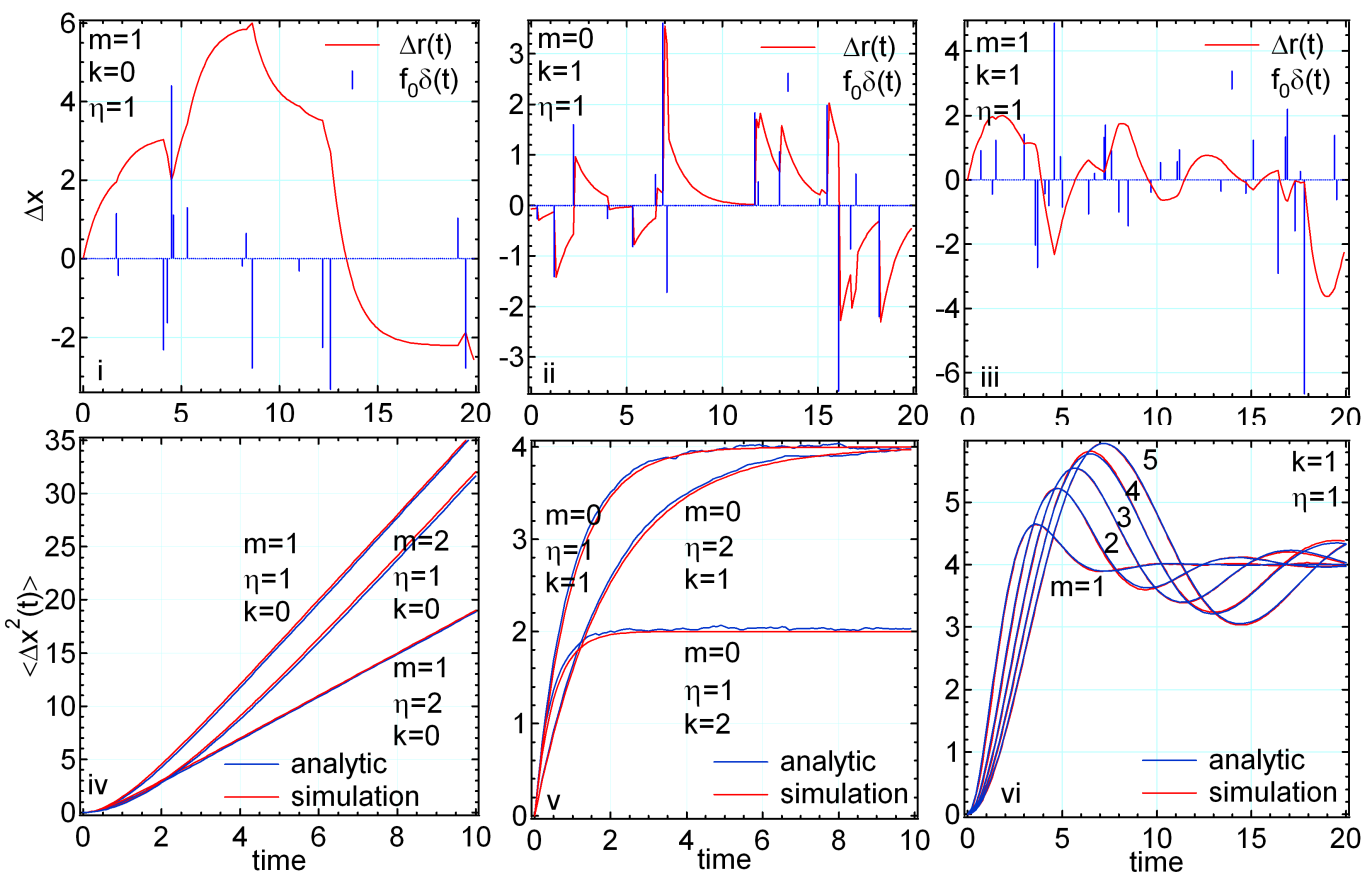

Figure 2.6: Simulated Brownian motion of a mass $m$ coupled to a Kelvin element of spring constant $k$ and dashpot viscosity $\eta$. i-iii) Simulated one-dimensional displacement and iv-vi) MSD. 
The physical meaning of the mean square displacement can be understood through simulation. The one-dimensional displacement of a test particle of mass $m$ coupled in series to a Kelvin element can be calculated for input impulse $f(t)=f_{0} \delta(t)$ representing Brownian forces using Laplace transform techniques. The amplitude and timing of the impulses are randomly chosen such that noise power is $F_{0}(\omega) F_{0}^{*}(\omega)=4 k_{B} T \Re e[Z(\omega)]=4 \zeta$ $\mathrm{J} / \mathrm{s}\left(k_{B} T=1 \mathrm{~J}\right)$. The simulated displacement of the test particle for various combinations of $m$ (in $\mathrm{kg}$ ), $\eta$ (in Pa.s) and $k$ (in Pa) is shown in Fig. 2.4 i-iii. The simulated MSD for an ensemble is shown below in Fig. 2.4 iv-vi. Analytic solutions are based on Eqn. 2.44. As expected for $k=0$ the MSD initially grows quadratically and then linearly at longer times. For $k \neq 0$ the MSD is observed to plateau. The spring element in the Kelvin circuit effectively tethers the test particle to its initial location preventing it from wandering off. If the particle is not massless, the resulting MSD shows a damped oscillation about a plateau value.

If $\left\langle\Delta r^{2}(t)\right\rangle \propto t$ as in Fig. 2.6 iv the diffusion is said to be 'free'. If however $\left\langle\Delta r^{2}(t)\right\rangle \propto t^{p}$, where $p<1$ as in Fig. 2.6 v-vi, the diffusion is said to be 'restricted' and sub-diffusive. Restricted diffusion can develop for purely geometric reasons. This is illustrated in Fig. 2.7. In the first example, the motion of a freely diffusing test particle in the interstitial void between a two-dimensional lattice of discs is considered. The radii of the discs is varied slightly. When the discs touch $(2 R=d)$ the test particle is contained within a single void. When a gap between the discs is present $(2 R<d)$ the test particle can escape into other voids. In all cases, at short times the $\left\langle\Delta r^{2}(t)\right\rangle \propto t$ - diffusion is free. At longer times differences develop. In the case where the discs touch, diffusion is completely restricted $\left\langle\Delta r^{2}(t)\right\rangle \propto t^{0}$. At intermediate times where $2 R<d$ sub-diffusive behaviour is observed. At long times, free diffusion $\left\langle\Delta r^{2}(t)\right\rangle \propto t$ is apparent with an effective diffusion constant $D$ that depends on $R$. In the second example, the free diffusion of a test particle that is trapped with a three-dimensional sphere is considered. The motion of the test particle is illustrated in Fig. 2.7 ii. The MSD of the test particle is shown in two cases in Fig. 2.7 iv. In the first case the cavity is fixed. Here, the diffusion is completely restricted at long times. In the second case, the cavity is assumed to diffuse freely with a much lower diffusion constant. In this case, the MSD of the test particle is essentially that of the cavity at long times. As a prelude to the next section a third example is shown in Fig. 2.7 iii. Here a hot snake thrashes in a long confining tube. Fig. 2.7 vi shows the schematic MSD of some point on the snake. At short times, the snake thrashing is unimpeded by the wall of the tube. Here the diffusion is free. At intermediate times, the wall restricts the thrashing motion. If a component of the thrashing motion is along the tube, the snake will gradually slither (or reptate) up and down the confining tube with a diffusion constant much lower than observed at short times. Clearly, a longer snake is going to have a harder time moving up and down the tube and its diffusion constant at long times will be lower. 

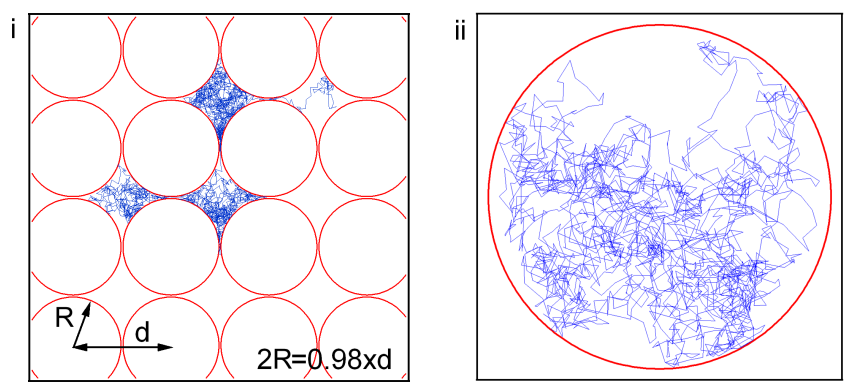

iii
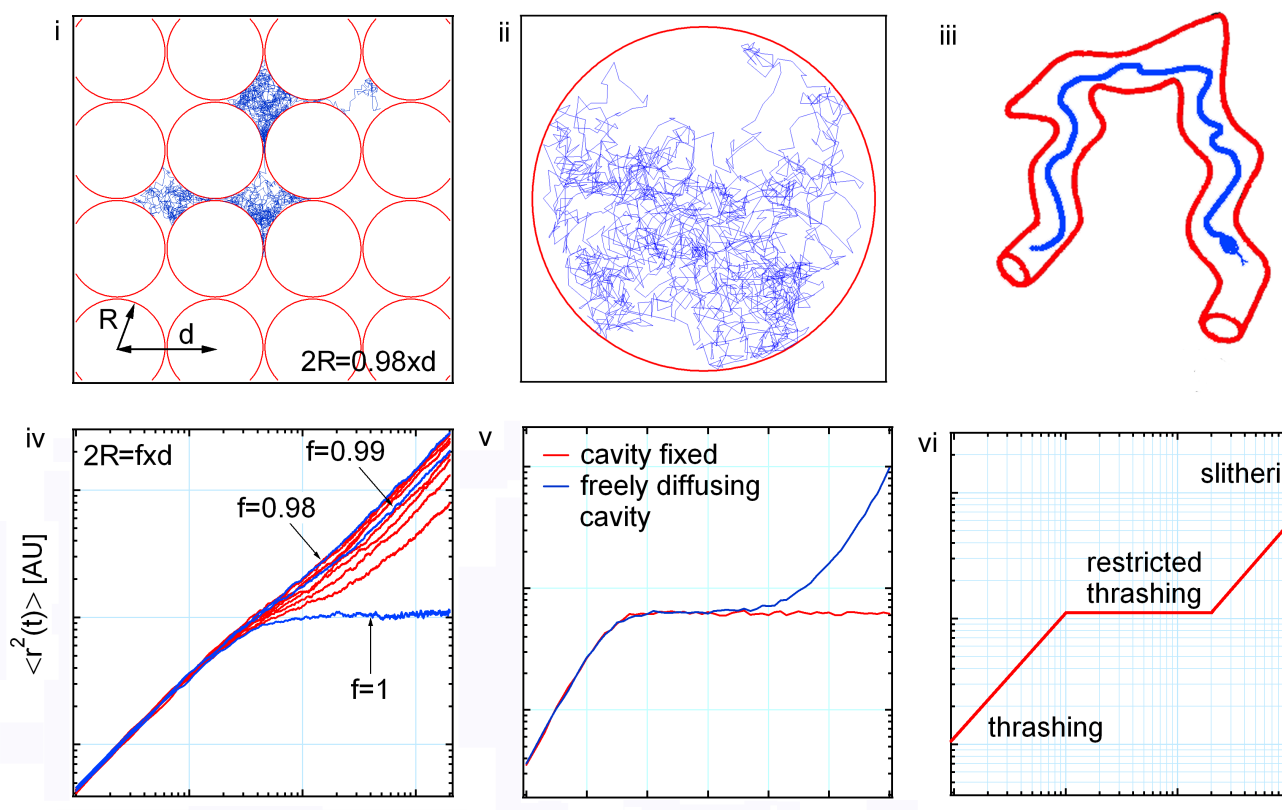

time $[\mathrm{AU}]$

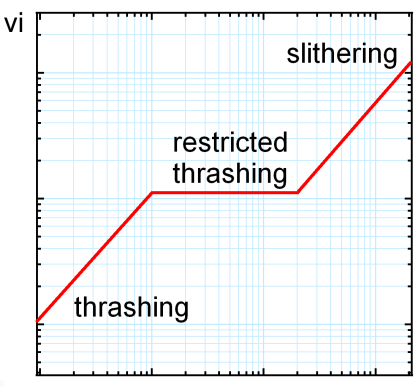

time $[\mathrm{AU}]$

Figure 2.7: Simulated 'Brownian' motion of freely diffusing test particles in restricted geometries. i and iii) Simulated two-dimensional displacement and MSD of test particles diffusing in the interstitial voids between a lattice of discs. ii and iv) Simulated threedimensional displacement and MSD of test particles diffusing in a spherical cavity. iii) A hot snake tries to escape from a confining tube. vi) A schematic representation of the MSD of some point on the hot snake. 


\subsection{The rheology of polymer-like chains}

\subsubsection{Structure}

Polymers are a linked series of repeating units called monomers. In a linear polymer, the monomeric units are linked in a chain. The conformation of the polymer chain in solution depends on both the polymer and the quality of the solvent (when present). For an ideal polymer composed of $N$ monomers of length $l$ the chain conformation is described by a random walk of step size $l$, step number $N$ and length $L=N l$. When bond angles between the monomers are restricted or if the solvent is not ideal (causing the polymer chain to swell or contract) the chain conformation is not that of a random walk. It can, however, still be described by an equivalent ideal polymer composed of $N_{K}(<N)$ monomers of length $l_{K}(>l)$ of total length $L=N_{K} l_{K}$ for which the conformation is that of a random walk. $l_{K}$ is called the Kuhn length which is half the persistence length $l_{p}$ - the characteristic length scale over which the chain can be considered rigid 8 .

The (effective) random walk of an individual polymer chain through some medium can be characterised by an end-to-end displacement vector $\mathbf{R}$; an ensemble can be characterised by the distribution function $\psi(\mathbf{R})$. In equilibrium conditions $\psi(\mathbf{R})$ is 8

$$
\psi(\mathbf{R})=\left[\frac{3}{2 \pi N l^{2}}\right]^{3 / 2} \exp \left[-\frac{3 \mathbf{R} \cdot \mathbf{R}}{2 N l^{2}}\right]=\left[\frac{\beta}{\sqrt{\pi}}\right]^{3} \exp \left[-\beta^{2} R^{2}\right] .
$$

where $\beta=\sqrt{3 /\left(2 N l^{2}\right)} \cdot \psi(\mathbf{R})$ is the probability of a particular end-to-end vector occurring (see Fig. 2.8). Increasing $R$ reduces the range of conformations that the random walk can assume and reduces the polymer's total entropy $S$. Consequently, work $W$ is required to stretch a polymer chain. From thermodynamic considerations $W=T S=k_{B} T \beta R^{2}$ and the force required $\mathbf{f}=\partial W / \partial \mathbf{R}=2 k_{B} T \beta \mathbf{R}$ indicating that an extended polymer acts like a Hookean spring for small deformations/forces [8].

At low concentrations, the polymer strands are isolated and rheology of the 'dilute' polymer solution is close to that of the solvent. At higher concentrations, the strands begin to overlap and become entangled. Because this 'overlap concentration' is typically low the solutions are referred to as 'semi-dilute'. The rheology of a semi-dilute polymer solution is significantly different to that of the solvent. When no solvent is present a polymer forms a 'melt'.

\subsubsection{Rubbery- and temporary-network models}

The rubbery-network model treats an entangled polymer solution as a network of springs. The relationship between stress and deformation for an ensemble of such springs can be determined by considering the force $\mathbf{f}$ on a surface embedded cube [5] [12]. Assume that the number density of springs is $\nu$ and the volume of the cube is $1 / \nu$ so that the cube on average contains a single spring (see Fig. $2.9 \mathrm{i}$ ). The probability that the spring will 

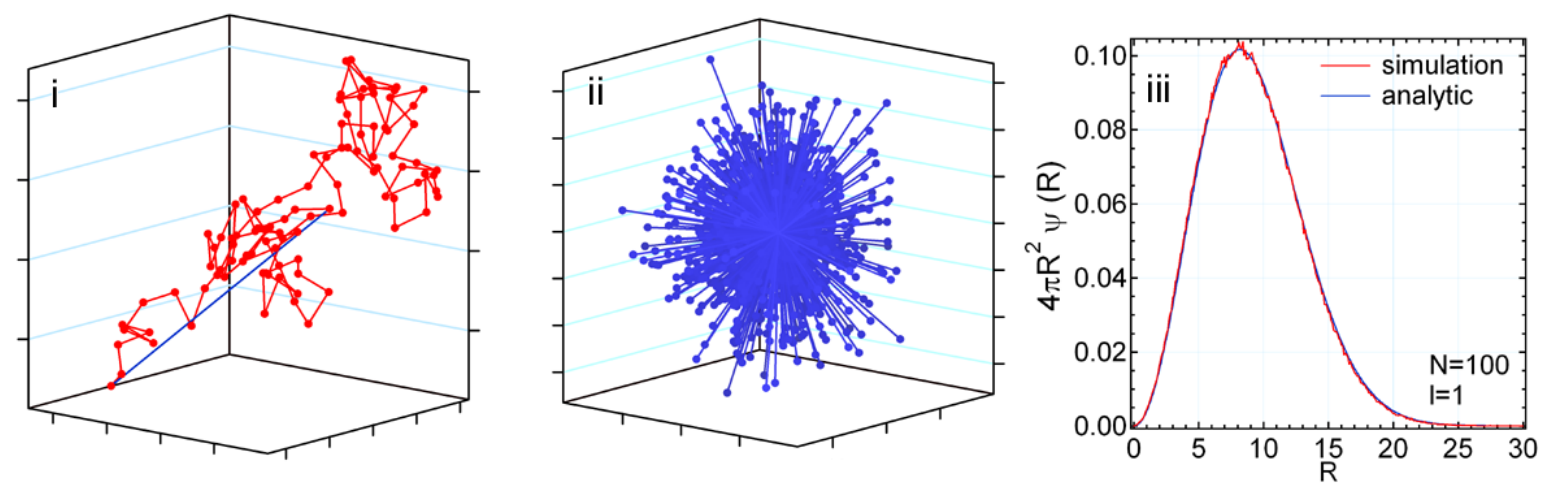

Figure 2.8: i) A random walk of 100 steps representing the conformation of a linear polymer. This conformation is characterised by an end-to-end displacement vector $\mathbf{R}$ (blue). The vector $\mathbf{R}$ can be ii) simulated for many such conformations and the iii) radial distribution of the end-to-end vector lengths $4 \pi R^{2} \psi(R)$ calculated.

intersect the surface with normal $\mathbf{n}$ is $\mathbf{R} \cdot \mathbf{n} \nu^{1 / 3}$. The average force on the surface of the cube is

$$
\mathbf{f}=\int \mathbf{R} \cdot \mathbf{n} \psi(\mathbf{R}) \mathbf{R} d R^{3}=2 \nu k_{B} T \beta \mathbf{n} \cdot\langle\mathbf{R R}\rangle
$$

where $\psi(\mathbf{R})$ describes the (possibly non-equilibrium) distribution of $\mathbf{R}$. Eqn. 2.52 has the form $\mathbf{f}=\mathbf{n} \cdot \underline{\underline{\sigma}}$ implying that $[5]$

$$
\underline{\underline{\sigma}}=2 \nu k_{B} T \beta^{2}\langle\mathbf{R R}\rangle
$$

The tensor $\langle\mathbf{R R}\rangle$ describes the average arrangement of the end-to-end vectors in the network. For a simple shear deformation of the form $R_{x} \rightarrow R_{x}+\gamma R_{y}$

$$
\begin{aligned}
& \langle\mathbf{R R}\rangle= \\
& {\left[\begin{array}{ccc}
\left\langle\left(R_{x}+\gamma R_{y}\right)^{2}\right\rangle & \left\langle R_{y}\left(R_{x}+\gamma R_{y}\right)\right\rangle & \left\langle R_{z}\left(R_{x}+\gamma R_{y}\right)\right\rangle \\
\left\langle\left(R_{x}+\gamma R_{y}\right) R_{y}\right\rangle & \left\langle R_{y} R_{y}\right\rangle & \left\langle R_{z} R_{y}\right\rangle \\
\left\langle\left(R_{x}+\gamma R_{y}\right) R_{z}\right\rangle & \left\langle R_{y} R_{z}\right\rangle & \left\langle R_{z} R_{z}\right\rangle
\end{array}\right]=\frac{R^{2}}{3}\left[\begin{array}{ccc}
1+\gamma^{2} & \gamma & 0 \\
\gamma & 1 & 0 \\
0 & 0 & 1
\end{array}\right] .}
\end{aligned}
$$

Note that it has been assumed that $\left\langle R_{i} R_{j}\right\rangle=\delta_{i j}\left\langle R_{i}^{2}\right\rangle=\left\langle R^{2}\right\rangle / 3$ (the average product of two uncorrelated Gaussian variables is zero). Eqn. 2.52 and 2.54 imply that [5]

$$
\sigma=\nu k_{B} T \underline{\underline{C}}^{-1}=G_{e} \underline{\underline{C}}^{-1}
$$

indicating that the Rubbery Network model describes a Hookean solid. Polymer solutions and melts are generally not 'solid'. Viscous behaviour is injected into the Rubbery Network 

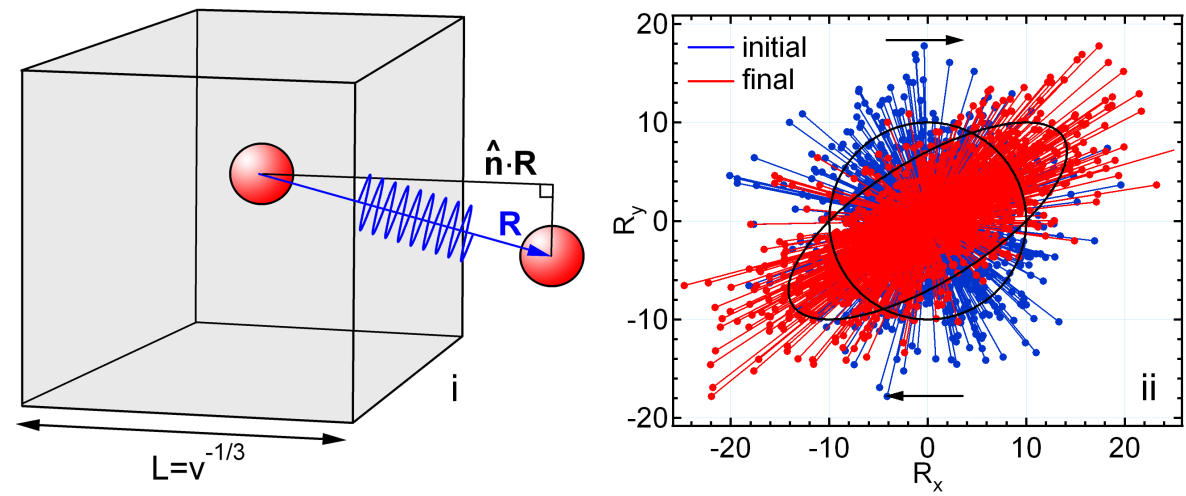

Figure 2.9: i) Geometric construction used to understand the stress-strain relationship in a rubbery-network. ii) Distribution of the end-to-end vectors $\mathbf{R}$ before and after deformation of the rubbery-network.

in the Temporary Network model. Here it is assumed that each spring in the network will break and recombine in the interval $t-t^{\prime}$ with a probability $P\left(t-t^{\prime}\right)$ given by $d P / d t=-P / \tau$. This leads to memory function of the form [5]

$$
m\left(t^{\prime}-t\right)=\frac{G_{e}}{\tau} \exp \left[\left(t^{\prime}-t\right) / \tau\right]
$$

By comparison with Eqn. 2.20 it is seen that the temporary-network model describes a Maxwell fluid. The nonlinear rheology predicted by the temporary-network model can be calculated by substituting this memory function into Eqn. 2.21 [5]

$$
\underline{\underline{\sigma}}(t)=\int \frac{G_{e}}{\tau} \exp \left[\left(t^{\prime}-t\right) / \tau\right] \underline{\underline{C}}^{-1}\left(t, t^{\prime}\right) d t^{\prime}
$$

\subsubsection{Reptation model}

In complex fluids, the energy stored as the result of a deformation is dissipated or 'relaxed' by diffusion. In the reptation model, an entangled polymer strand is thought of as being trapped within a tube or 'primitive path' formed by the topological constraints produced by surrounding polymers. This primitive path is illustrated schematically in Fig. 2.10. Entropy is recovered by curvilinear diffusion of the strand along the tube. From the statistics of a random walk, for a polymer melt composed of linear polymers of length $L=l N$, the tube diameter and distance between entanglements is $l_{e}=l \sqrt{N_{e}}$ and average tube length is $L_{e}=L / \sqrt{N_{e}}$ where $N_{e}$ is the number of monomers between 'entanglement' points. These entanglements impede the diffusion of the chain. By considering the time required for a strand to diffuse out of the tube, Doi and Edwards calculated the stress 

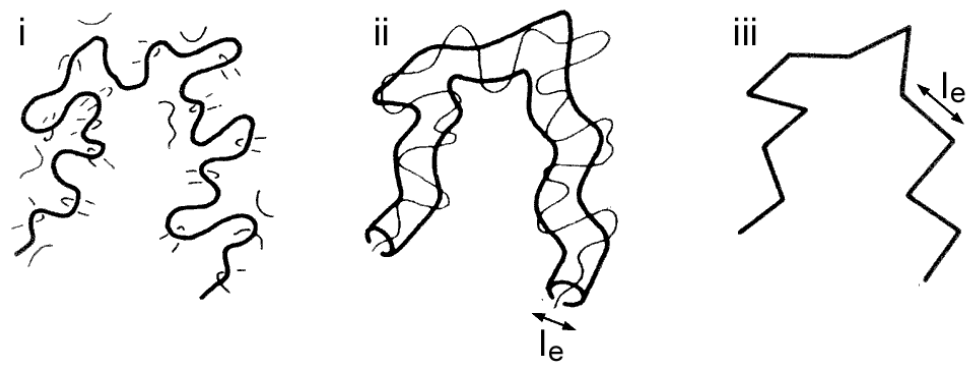

Figure 2.10: The topological constraints on an i) entangled polymer strand can be represented by a ii) confining tube which is itself a random walk or iii) primitive path through the fluid (adapted from [5]).

relaxation should follow [8]:

$$
\begin{aligned}
G(t) & =G_{e} \frac{8}{\pi^{2}} \sum_{p, o d d}^{\infty} \frac{1}{p^{2}} \exp \left[p^{2} t / \tau_{r e p}\right] \\
G^{*}(\omega) & =G_{e} \frac{8}{\pi^{2}} \sum_{p, o d d}^{-\infty}\left[\frac{\omega^{2} \tau_{r e p}^{2}}{p^{2}\left(p^{4}+\omega^{2} \tau_{r e p}^{2}\right)}+i \frac{\omega \tau_{r e p}}{p^{4}+\omega^{2} \tau_{r e p}^{2}}\right] .
\end{aligned}
$$

In a melt the strands are space filling. The number density $\nu$ is independent of the length of the polymer chain. $\tau_{r e p}=L^{2} / D_{r e p}$ is the characteristic time required for the strand to 'reptate' or diffuse out of the original confining tube. The diffusion constant $D_{\text {rep }}$ is inversely proportional to the number of monomers in the chain. This leads to the important prediction that $\tau_{r e p} \propto l_{e}^{2} N^{3} / N_{e}$. Eqn. 2.58 (illustrated in Fig. 2.11) is dominated by the lowest mode Maxwell element. Earlier it was seen that for a Maxwell fluid $\eta=G \tau$. In the approximation that the contribution of higher order Maxwell modes can be neglected, which is only appropriate at low or 'zero' strain rate, the 'zero' shear viscosity, $\eta_{0}=G_{e} \tau_{r e p} \propto N^{3}$. This is close to the experimental value of $\eta_{0}=N^{3.4}$ (perhaps the most famous result in all of soft matter physics). In a semi-dilute polymer solution, relaxation also occurs through reptation. Here the basic predictions for a polymer melt can be modified to include concentration effects. In a semi-dilute polymer solution of volume fraction $\phi_{V}=V_{\text {poly }} /\left(V_{\text {poly }}+V_{\text {solv }}\right), G_{e} \propto \phi_{V}^{2.3}, \tau_{r e p} \propto N^{3} k_{B} T \phi_{V}^{1.6-2.4}$ and $\eta_{0}=$ $G_{e} \tau_{r e p} \propto N^{3} \phi_{V}^{3.9-4.7}[8]$.

The associated memory function of the entangled polymer-like chain is [5]

$$
m\left(t-t^{\prime}\right)=G_{e} \frac{8}{\pi^{2}} \sum_{i, o d d} \frac{1}{\tau_{r e p}} \exp \left[p^{2}\left(t-t^{\prime}\right) / \tau_{r e p}\right]
$$

Doi and Edwards determined that the stress tensor for a reptating polymer has the form 

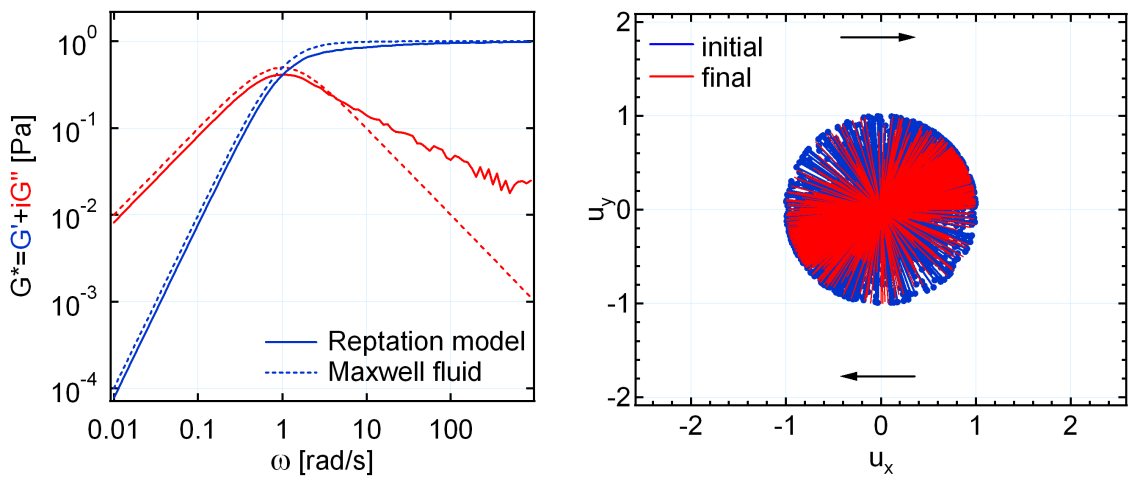

Figure 2.11: i) Complex shear modulus of an entangled polymer melt or solution (from Eqn. 2.59 with a comparison to that of a Maxwell fluid. ii) Distribution of the primitive path bond vectors $\mathbf{u}$ (including retraction) before and after deformation.

5

$$
\underline{\underline{\sigma}}(t)=G_{e} \frac{8}{\pi^{2}} \int_{-\infty}^{t} \sum_{i, o d d} \frac{1}{\tau_{r e p}} \exp \left[\left(p^{2}\left(t-t^{\prime}\right) / \tau_{r e p}\right] \underline{\underline{Q}}\left(t, t^{\prime}\right) d t^{\prime}\right.
$$

$\underline{\underline{Q}}\left(t, t^{\prime}\right)$ is called the universal strain tensor which is finger tensor $\underline{\underline{C}}^{-1}\left(t, t^{\prime}\right)=\langle\mathbf{R R}\rangle$ modified to include tube retraction effects. Retraction prevents the bond vectors $\mathbf{u}$ of the primitive path from changing length during deformation so that $\mathbf{u}=\mathbf{u}^{\prime} \cdot \underline{\underline{F}}^{-1} /\left|\mathbf{u}^{\prime} \cdot \underline{\underline{F}}^{-1}\right|$ and [5]

$$
\underline{\underline{Q}}=\langle\mathbf{u} \mathbf{u}\rangle=\left\langle\frac{\mathbf{u}^{\prime} \cdot \underline{\underline{F}}^{-1} \mathbf{u}^{\prime} \cdot \underline{\underline{F}}^{-1}}{\left|\mathbf{u}^{\prime} \cdot \underline{\underline{F}}^{-1}\right|^{2}}\right\rangle .
$$

The effect of a deformation on the bond vectors of a primitive path is shown in Fig. 2.11 ii. Deformation rotates the vectors without extension. For a given deformation $\underline{\underline{F}}^{-1}\left(t, t^{\prime}\right)$, the universal strain tensor $Q\left(t, t^{\prime}\right)$ can be evaluated. The behaviour of $Q_{12}\left(t, t^{\prime}\right)$ for deformations $\underline{\underline{F}}_{12}^{-1}\left(t, t^{\prime}\right)=H\left(t^{\prime}\right) \dot{\gamma} t^{\prime}$ (where $H\left(t^{\prime}\right)$ is the Heaviside step function) is evaluated using simulation and analytically using the Currie approximation [5] in Fig. 2.12 i. By multiplying by the memory function and integrating, the stress as a function of time, can be determined. The equilibrium stress and the viscosity $\eta=\sigma / \dot{\gamma}$ is evaluated for a number of strain rates in Fig. 2.12 ii. Notably, it is seen that the viscosity decreases with increasing strain rate indicating that a polymer melt or semi-dilute polymer solution is shear thinning. It is also seen that the stress is not monotonic with increasing strain-rate. 

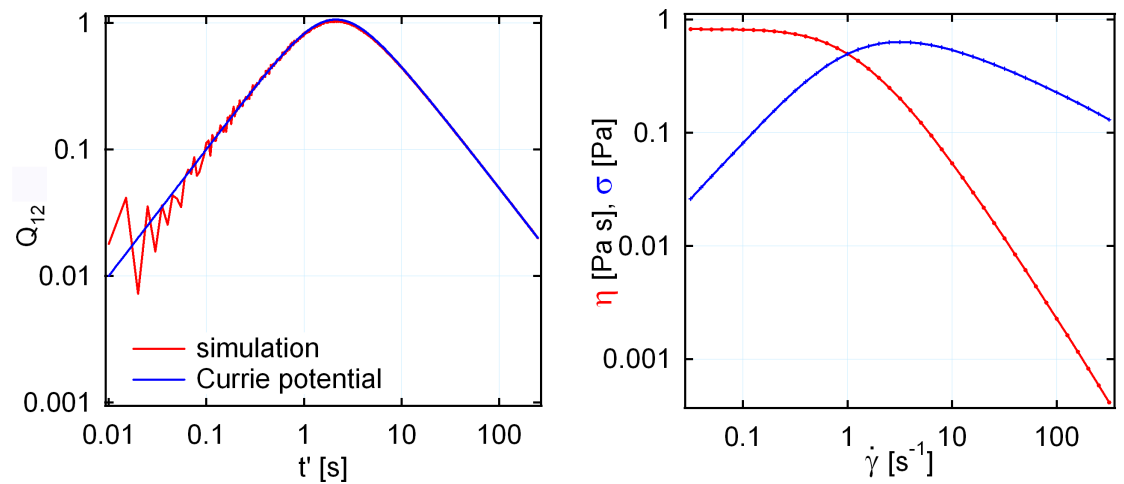

Figure 2.12: i) A comparison of the $Q_{12}$ component of the universal strain tensor for a strain that increases linearly with time $t^{\prime}$ evaluated using both simulation and the Currie potential approximation. ii) With $Q_{12}$ determined, the steady-state viscosity and stress with stain rate for a monodisperse polymer melt or semi-dilute polymer solution can be calculated.

\subsubsection{Other relaxation modes}

Reptation is the dominant relaxation mode at low frequency. At higher frequencies other relaxation modes become important. In the Rouse model a polymer chain is treated as $N$ Kuhn segments coupled together by Hookean springs. Like the string of a guitar, the motion of the system can be decomposed into $N$ normal modes, with each normal mode representing the motion of the strand on length scale $L, L /(N / 2), L /(N / 3) \ldots L / p \ldots L / N$. When perturbed, each mode stores energy of the order of $3 k_{B} T / 2$ and the total energy stored by the chain is of the order $N k_{B} T$. As with reptation, the stored energy in each mode is dissipated by Brownian motion. Each mode satisfies an equation of motion $k_{p} x+$ $\zeta_{p} \dot{x}=f(t)$ where $k_{p}$ is the restoring force and is dependent on the order of the mode with $k_{p} \propto p^{2}$ and $\zeta_{p}$ represents the viscous drag with $\zeta_{p}=2 N \zeta$. The average time dependence of the relaxation process can be determined from the position autocorrelation $\left\langle x_{p}(0) x_{p}(t)\right\rangle$. The correlation $\left\langle x_{p}(0) x_{p}(t)\right\rangle$ is calculated from the simulated $x(t)$ variation for thermal excitation of the Kelvin element shown in Fig. 2.6 (with $m=0$ ) and compared to a function of the form $y=\exp [-t k / \eta]$. Clearly this relaxation is monoexponential. The relaxation over the entire chain is the sum of relaxations of all the modes. This normalised sum is shown in Fig. 2.13 i for $N=1000$. The stress relaxation $G(t)$ is proportional to this sum [8]:

$$
\begin{aligned}
G(t) & =G_{0} \sum_{p=1}^{N} \exp \left[-\frac{k_{p}}{\zeta_{p}} t\right]=G_{0} \sum_{p=1}^{N} \exp \left[-\frac{t}{\tau_{p}}\right], \\
G^{*}(\omega) & =G_{0} \sum_{p=1}^{N}\left[\frac{\omega^{2} \tau_{p}^{2}}{1+\omega^{2} \tau_{p}^{2}}+i \frac{\omega \tau_{p}}{1+\omega^{2} \tau_{p}^{2}}\right],
\end{aligned}
$$


where $\tau_{p}=\left(l^{2} N^{2} \zeta\right) /\left(6 \pi^{2} k_{B} T p^{2}\right)$ and $G_{0}=\left(k_{B} T \phi\right) /\left(N l^{2}\right)$. Eqn. 2.63 is the Rouse mode relaxation of a semi-dilute solution polymer chain at a volume $V$ fraction of $\phi=$ $V_{\text {poly }} / V_{\text {poly }+ \text { solv }}$. The associated complex shear modulus $G^{*}(\omega)$ is shown in Fig. 2.13 ii. The lowest mode $\tau_{R}=\tau_{p=1}$ is called the Rouse characteristic time and is related to the $\tau_{\text {rep }}$ by [8]

$$
\tau_{\text {rep }}=6 \tau_{0} \frac{N^{3}}{N_{e}}=6 \tau_{e}\left(\frac{N}{N_{e}}\right)^{3}=6 \tau_{R} \frac{N}{N_{e}} .
$$

$\tau_{0}$ is the characteristic time for a monomer to diffuse a Kuhn length $l_{K}$. $\tau_{e}$ is the characteristic time for a monomer to diffuse the distance between entanglement points on the tube. $\tau_{R}$ is the characteristic time required for a monomer to freely diffuse a distance equal to the size of the polymer. $\tau_{r e p}$ is greater than $\tau_{R}$ by a factor $N / N_{e}$ - a longer snake (bigger $N$ ) has a harder time get getting out of the confining tube.

In the original reptation model, the length of the confining tube was assumed to be fixed. However the length of the tube will vary as the polymer strands forming the tube themselves reptate. These tube-length fluctuations introduce another mode of relaxation. Tube length fluctuations or breathing modes most significantly affect reptation at short times 8 ]

$$
G(t)=G_{e}\left[1-\frac{N_{e}}{N}\left(\frac{t}{\tau_{e}}\right)^{1 / 4}\right], \quad t<\tau_{R} .
$$

The effect of these breathing modes is illustrated in Fig. 2.13 iii. The effect of breathing modes on the complex shear modulus is determined in Fig. 2.13 iv by numerical integration. Breathing modes reduce the apparent $G_{e}$ slightly and introduce a $G^{\prime \prime} \propto \omega^{-1 / 4}$ dependence at high frequency. These tube length fluctuations account for the experimental observation that $\eta_{0} \propto N^{3.4}$ and not $\eta_{0} \propto N^{3}$ as predicted by the original Doi-Edwards reptation model [8].

The total relaxation is the sum of all relaxation modes. At low frequency, the behaviour is dominated by reptation. At high frequency, Rouse mode relaxation is most important. The effect of high frequency modes on the nonlinear rheology is generally neglected. 

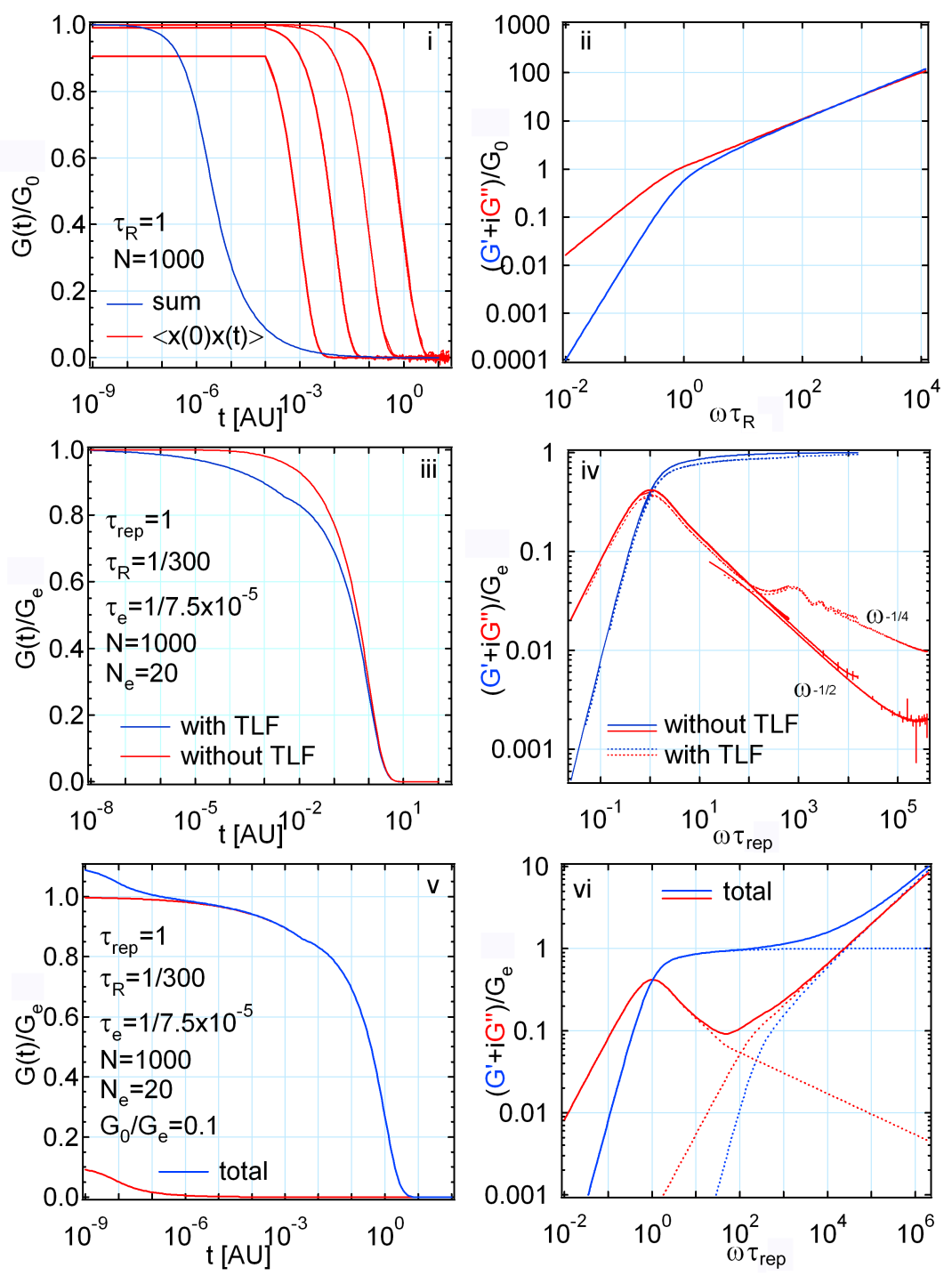

Figure 2.13: The effect of high frequency relaxation modes on the linear rheology of polymer solutions/melts. i-ii) Rouse modes, iii-iv) tube length fluctuations (TLF or breathing modes) during reptation and v-vi) both Rouse and tube length fluctuations during reptation. 


\section{Chapter 3}

\section{Rheology of wormlike micelles solutions}

\subsection{Introduction}

Wormlike micelles are a polymer-like self-assembly of surfactant molecules that can form in solution in the appropriate conditions. Unlike linear polymers where length is fixed at polymerisation, wormlike micelles are formed dynamically through a continuous process of breakage and recombination. Wormlike micelles solutions possess several unusual rheological properties and have been the subject of active study for over 20 years. In this chapter the general properties of surfactants in solution are reviewed. A widely accepted description of the low frequency linear rheology of wormlike micelles called the 'reptationreaction' model is introduced. The 'Poisson-renewal' model is advanced to include high frequency modes and experimental evidence for this model is considered. The nonlinear rheology predicted on the basis of the reptation-reaction model is described. Evidence for a particular prediction that some wormlike micelles solutions may 'shear-band' is reviewed. The role of coupling between flow and concentration, and flow instabilities and stress fluctuation are discussed in the context of JS, dJS and dJS $\phi$ continuum models, along with experimental evidence.

\subsection{Surfactants in solution}

Surfactants are amphiphilic molecules composed of a more soluble lyophilic (solvent loving) component and less soluble lyophobic (solvent hating) part(s). Contact between the lyophobic components of the surfactant and the solvent is energetically unfavourable. As a consequence, surfactants (a contraction of 'surface acting agents') tend to adsorp at interfaces to minimise contact between these components.

The lyophobic part of a surfactant molecule or unimer is often a non-polar hydrocar- 


sodium stearate (major component of soap)

Table 3.1: Chemical structure of selected surfactant molecules.

bon chain, typically 8-18 carbon atoms long, and commonly referred to as the surfactant's 'tail'. Conversely the polar lyophilic part is called the head. Surfactants are classified by the charge on the head group. In anionic surfactants, the head carries a negative charge in solution. This kind of surfactant is an active ingredient in many detergents. By contrast, in a cationic surfactant the head carries a positive charge. While less common, they are of considerable interest because of their capacity to modify the chemistry of negatively charged surfaces and are often used in the preparation of wormlike micelles. Cationic surfactants are the primary focus of the experimental work presented here. In a nonionic surfactant, the head group carries no charge whereas in a zwitterionic surfactant the head group carries both positive and negative charges. The chemical structure of selected surfactant molecules is illustrated in Table 3.1.

At lower concentrations, surfactants exist as isolated unimers in solution. At higher concentrations, unimer saturation is achieved and the excess surfactant is reversibly self adsorbed to minimise contact between the lyophobic and lyophilic parts. The structures formed by the self-adsorption/self-assembly of surfactant molecules are called micelles and the concentration at which this occurs is called the critical micelle concentration. A schematic phase-diagram illustrating both the dependence on concentration and temperature is shown in Fig. 3.1 i. The lowest temperature and concentration at which micellisation occurs is called the Krafft point.

The structure of the micelles formed depends on the relative sizes of the head and tail and is characterised by the critical packing parameter $v /(l a)$ where $v$ is the surfactant volume, $l$ is the length of the tail and $a$ is the effective cross-sectional area of the head. Depending on the value of the critical packing parameter the micelles may be locally spherical, cylindrical or planar. The effective cross-sectional area of the head depends on electrostatic interaction between the heads. Electrostatic repulsion present in ionic surfactant solutions can be screened by the addition of salts which reduce the effective head size and increase the packing parameter. Charged counterion or short chain cosurfactants which incorporate directly into the body of the micelles can act as molecular 

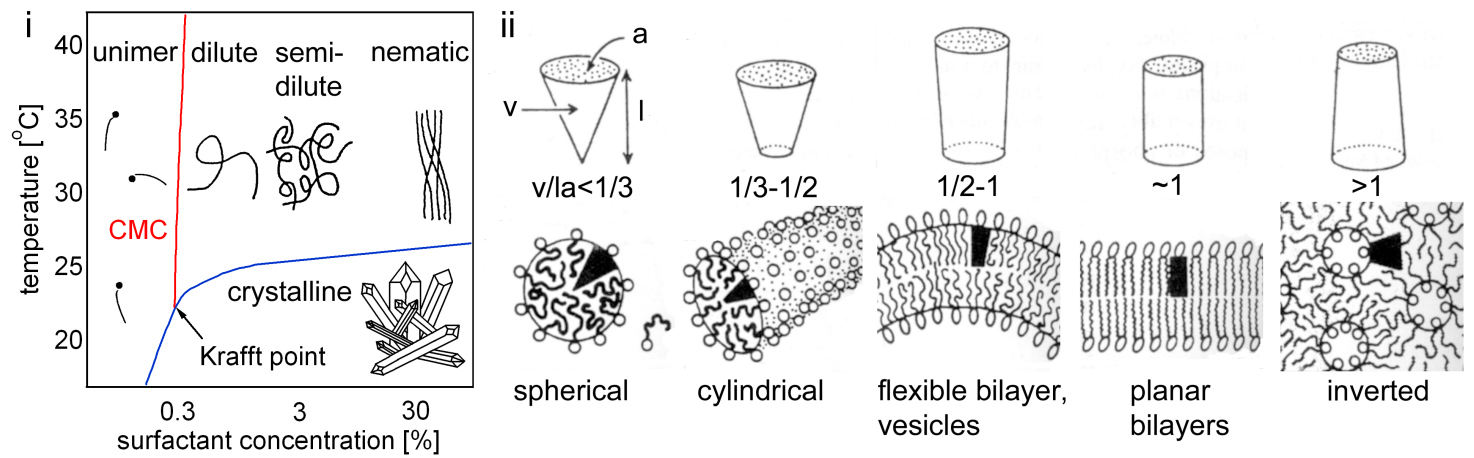

Figure 3.1: i) A schematic phase diagram showing the state of the surfactants in solution and ii) dependency of the micelle mesostructure on the critical packing parameter (adapted from [4]).

'spacers' between the unimers and may also be used to modify the packing parameter [4].

Cylindrical micelles have attracted considerable interest in the last 20 years. These semi-flexible 'wormlike' micelles have a radius equal to the fully extended length of the surfactant chain and may be microns long. At lower or 'dilute' concentration the wormlike micelles are unentangled. At intermediate or 'semi-dilute' concentrations the micelles overlap and become entangled. At higher concentrations $(\phi=30-40 \%)$ the cylindrical micelles begin to form an ordered liquid crystalline-like nematic phase. This progression is shown schematically in Fig. 3.1 i.

Structurally, wormlike micelles are similar to linear polymers. Unlike linear polymers where polymer length is set at polymerisation, wormlike micelles are formed dynamically through a continuous process of 'breakage and recombination' or 'reversible scission'. Because of this dynamic behaviour, wormlike micelles are sometimes known as 'living polymers'.

\subsection{Linear rheology of wormlike micelles}

\subsubsection{Reptation-reaction model}

Early rheological work in the 1980's often focused on the unusual viscoelastic response of the semi-dilute wormlike micelles solutions. In the appropriate conditions, some solutions show a nearly Maxwell-like linear response as is illustrated in Fig. 3.2. This behavior is not seen in polymer solutions/melts suggesting that it may be associated with reversible scission.

In 1987 [14 Cates investigated the effect of reversible scission on the linear rheology of entangled semi-dilute linear polymer-like solutions by simulation. In his simulation, it 

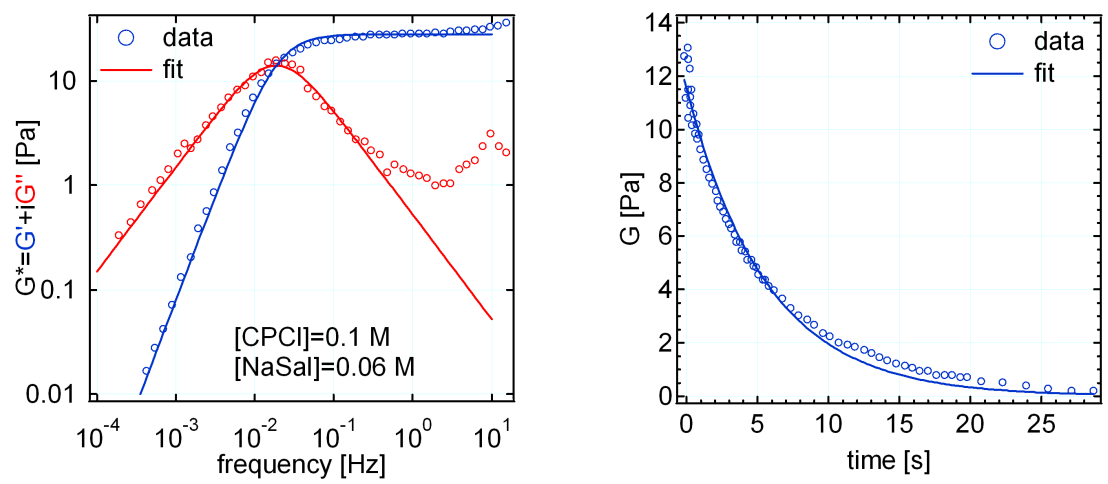

Figure 3.2: Maxwell-like response shown by a wormlike micelles solution (from [13]) in the i) frequency and ii) time domains.

was assumed that the primitive path/confining tube is composed of left and right segments of lengths $L_{L}$ and $L_{R}$ with a length of segment drawn randomly from an exponential distribution with mean $1\left(L_{L}=-\ln \phi\right.$ and $L_{R}=-\ln \phi$ where $\phi$ is drawn (separately for $L_{L}$ and $L_{R}$ ) from a uniform distribution on the interval [0,1]), of total length $L=L_{R}+L_{L}$ and average length $\bar{L}=1$. As implied by the statement $\bar{L}=1$, all lengths have been normalised with respect to the average length of the primitive path/confining tube so that the quantities $L_{L}, L_{R}, L, \bar{L}$ and $\Delta$ (below) are dimensionless. These segments evolve according to a characteristic reptation time $\tau_{r e p}=\bar{L}^{3} / D_{0}$ where $D_{0}$ is a constant and a characteristic reaction time $\tau_{b}$. In an interval $\delta t$ the left and right segments:

1. can break at arbitrary point, that is, $L_{L} \rightarrow L_{L} \times \phi$ or $L_{R} \rightarrow L_{R} \times \phi$, with probability $P=L_{L} \delta t / \tau_{b}$ or $P=L_{R} \delta t / \tau_{b}$,

2. can recombine with another segment with a length drawn from an exponential distribution with mean one, that is, $L_{L} \rightarrow L_{L}-\ln \phi$ or $L_{R} \rightarrow L_{R}-\ln \phi$ with probability $P=\delta t / \tau_{b}$ or $P=\delta t / \tau_{b}$

3. will diffuse either left or right a distance $\Delta=\sqrt{2 \delta t / \tau_{\text {rep }} L}$, that is, $L_{L} \rightarrow L_{L}+\Delta$ and $L_{R} \rightarrow L_{R}-\Delta$ or $L_{L} \rightarrow L_{L}-\Delta$ and $L_{R} \rightarrow L_{R}+\Delta$.

The steps above are iterated $i$ times until either $L_{L}<0$ or $L_{R}<0$ at which time the segment escapes through one end and the tube relaxes to give a relaxation time $t_{i}$. The typical evolution of the simulated path is shown in Fig. 3.3 i. This is repeated many time to give a distribution of relaxation times; this distribution of relaxation times is proportional to the memory function. From this memory function the stress relaxation can be calculated by integration.

In the 'slow break' limit $\tau_{b}>>\tau_{r e p}$ the simulation indicated that

$$
G(t) \approx G_{e} \exp \left[-\left(t / \tau_{r e p}\right)^{-0.25}\right] .
$$



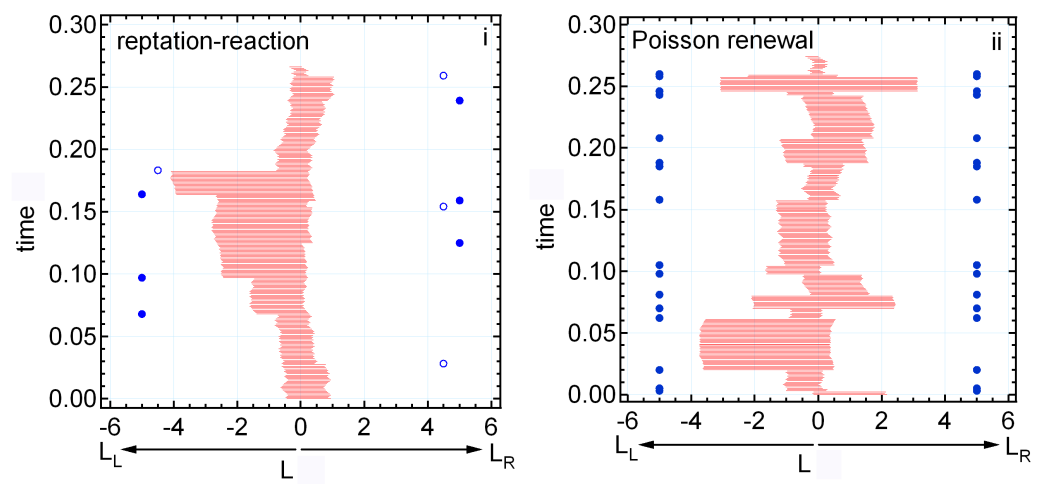

Figure 3.3: i) Simulated evolution of the primitive path with time, subject to breakage (open circles) and recombination (closed circles) and diffusion according to the reptationreaction model. ii) This can be compared to the (Author's conception of the) evolution of the primitive path subject to reinitialisation (closed circle) and diffusion according to the Poisson-renewal model. Rouse and breathing relaxation modes are not indicated.

This is the stress relaxation predicted by Doi and Edwards' reptation model for a semidilute exponentially polydisperse linear polymer solution demonstrating that Cates' simple simulation captures the essential physics of the reptation model. In the 'fast break' limit $\tau_{\text {rep }}>>\tau_{b}$

$$
G(t)=G_{e} \exp \left[-\left(t / \tau_{r}\right)\right]
$$

where $\tau_{r} \approx \sqrt{\tau_{b} \tau_{r e p}} . \quad \tau_{r}$ is called the terminal relaxation time. Remarkably, Eqn. 3.2 predicts that stress relaxation is monoexponential in this fast break limit and that the linear rheology is that of a Maxwell fluid as is observed experimentally in some solutions. The linear rheology predicted by the reptation-reaction model is shown in Fig. 3.4 in a number of cases. Fig. 3.4 ii shows a parametric representation of the data called a Cole-Cole plot. This representation emphasises the high frequency behaviour. This basic model was subsequently adapted [17, [18] to include several other processes including endinterchange and bond-interchange reactions. The effects of these new processes on the linear rheology are qualitatively similar to those seen by reversible scission as described above.

\subsubsection{Poisson-renewal model}

The reptation-reaction model excludes the effects of high frequency Rouse and breathing modes on the stress relaxation. These modes were found to be difficult to include in the simulation described in the previous section. In 1992, Granek and Cates subsequently described an analytic approach based on Poisson statistics that they called the Poisson-renewal model which described both low frequency linear rheology predicted by 

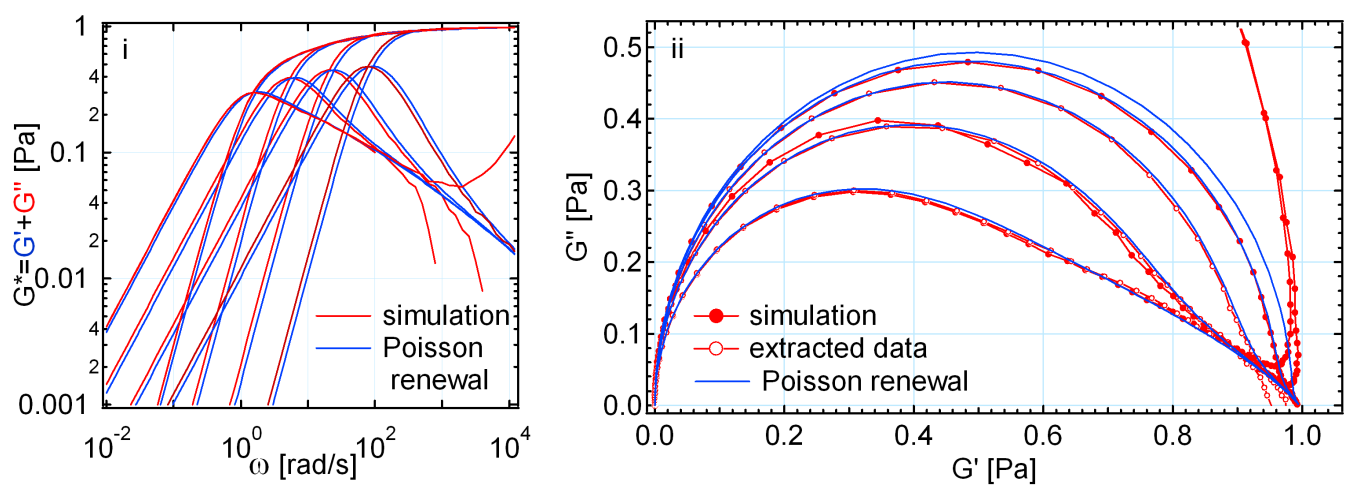

Figure 3.4: i) Comparison of the Author's own implementation of Cates' reptation-reaction simulation with an analytic calculation according to the Poisson-renewal model [15. ii) Cole-Cole representation of the complex shear modulus calculated using the Author's simulation and the Poisson-renewal model with a comparison to literature data [16].

the reptation-reaction model and could include the effect of higher frequency modes on stress relaxation [15]. The evolution of the primitive path according to the model is illustrated schematically in Fig. 3.3 ii. As with the reptation-reaction model, the primitive path diffuses with a diffusion constant inversely proportional to the length of the path. When the path escapes, the tube relaxes. In the reptation-reaction model, breakage and recombination significantly modifies the length of the path. This breakage and recombination is implemented in the Poisson-renewal model by randomly reassigning - or renewing - the length of the tube with a frequency that obeys Poisson statistics. On the interval between renewal events, stress relaxation occurs through reptation, Rouse and breathing modes. In the reptation-reaction model, there is some correlation between the length of the path before and after breakage and recombination. This correlation can be included in the Poisson- renewal model.

A full mathematical description of the model is given in [15]. It is noted that this description contains typographic errors (confirmed by Cates) - these have been corrected. The typical behaviour predicted by the Poisson-renewal model along with a comparison to a model literature data [15] is shown in Fig. 3.5. Note the turn-up at high frequencies shown in Fig. $3.5 \mathrm{i}$ - this turn-up is not predicted by the reptation-reaction model.

The Poisson-renewal model predicts that

$$
\frac{G_{\min }^{\prime \prime}}{G_{e x t}^{\prime}} \geq \frac{l_{e}}{\bar{l}}
$$

where $\bar{l}$ is the average length of the micelles in the exponentially polydisperse solution. $G_{\text {min }}^{\prime \prime}$ is the local minimum in $G^{\prime \prime}$ and $G_{\text {ext }}^{\prime}$ is the extrapolated value of $G^{\prime}$ at $G^{\prime \prime}=0$ prior to the turn-up in $G^{\prime \prime}$ on a Cole-Cole plot. This value is approximately equal to $G_{e}$. Eqn. 3.3 puts a lower limit on the ratio $l_{e} / \bar{l}$. The correlation length $\xi$ or 'mesh' size of the linear 

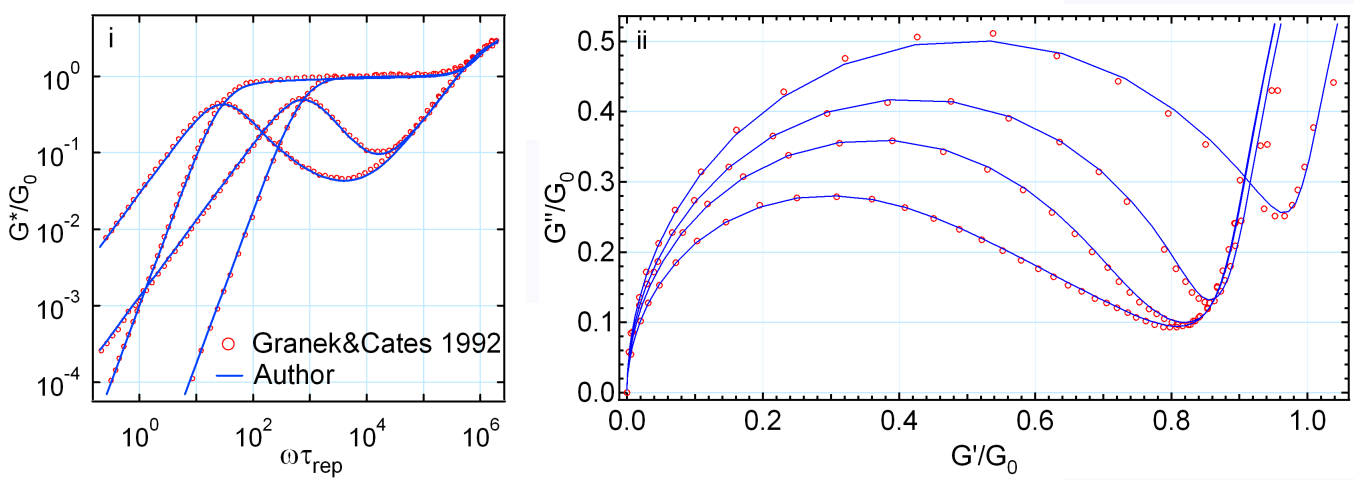

Figure 3.5: Typical linear rheology predicted by the Poisson-renewal model as i) a function of frequency and ii) a Cole-Cole plot showing a turn-up at high frequencies. A comparison of the Author's calculation to literature data [15] is included.

polymer network is

$$
\xi \simeq\left[\frac{k_{B} T}{G_{e}}\right]^{1 / 3}
$$

The persistence length $l_{p}$ is the characteristic length over which a polymer chain can be thought to be 'rigid' and is related to the entanglement length by

$$
l_{e} \simeq \xi^{5 / 3} / l_{p}^{2 / 3}
$$

If the ratio, $l_{e} / \bar{l}$ and $G_{e x t}$ can be measured and $l_{p}$ can be estimated, the average length of the micelles $\bar{l}$ can be determined. This value is difficult to determine otherwise. The techniques conventionally used to size polymers (such as those discussed in chapter 3) rely on the solutions being dilute.

The validity of the Poisson-renewal model is somewhat difficult to test. The mechanical inertia of a conventional rheometer begins to affect measurements of the complex shear modulus around $20 \mathrm{~Hz}$. Because most wormlike micelles solutions show characteristic Maxwell times in the range $0.1<\tau_{r}<1 \mathrm{~s}$ very little of the turn-up predicted by the Poisson-renewal model can be observed. An attempt to test the validity of the Poissonrenewal model is shown in Fig. 3.6 (from [19]). When presented as a parametric Cole-Cole plot, the measured data appears to match the behaviour predicted by the Poisson-renewal model quite well. However, when the data are replotted with frequency, a clear deviation from the behaviour predicted by the Poisson-renewal model is observed at higher frequencies. Inertia of the instrument may be affecting measurements at high frequency or the model may not accurately describe the measured high frequency behaviour. 

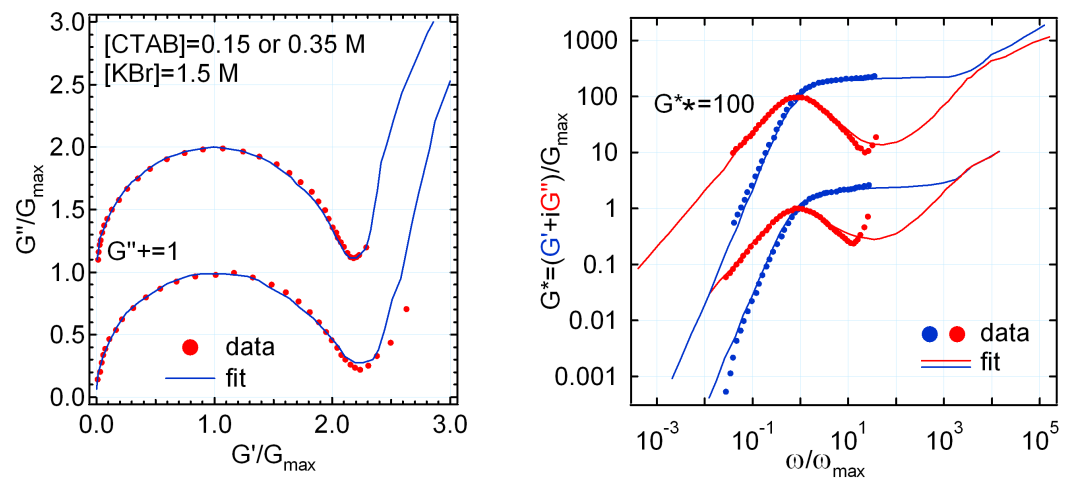

Figure 3.6: Measured $G^{*}(\omega)$ for two wormlike micelles solutions with a comparison to a 'best-fit' calculated according to the Poisson-renewal model [19] depicted as i) a Cole-Cole plot and ii) as a function of frequency more clearly showing a mismatch at high frequencies.

\subsection{Nonlinear rheology of wormlike micelles}

\subsubsection{Shear banding}

Shear thinning is a common feature of the nonlinear rheology of entangled polymer solutions/melts and is closely associated with reptation. Shear thinning is often exhibited by wormlike micelles solutions. In some instances, the thinning is so extreme that the equilibrium stress is actually constant with increasing strain rate appearing as a 'stress plateau' in the flow curve. This behaviour is illustrated in Fig. 3.7 i. This extreme shear thinning is generally not seen in entangled polymer solutions/melts suggesting that reversible scission may be influencing the nonlinear flow behaviour of wormlike micelles.

By adapting the constitutive model for entangled polymer solutions to include the effects of reversible scission, Cates postulated that the constitutive relationship was given by

$$
\underline{\underline{\sigma}}(t)=\frac{15}{4} G_{e}\left[\int_{0}^{\infty} \mathcal{B} \exp \left[t-t^{\prime}\right] \mathcal{D} \underline{\underline{Q}}\left(t-t^{\prime}\right)-\frac{1}{3} \underline{\underline{I}}\right]
$$

in 1990 [20]. $\mathcal{B}$ and $\mathcal{D}$ are functions of the 'birth' and 'death' rates of the wormlike micelles, $\underline{\underline{Q}}$ is the universal strain tensor and $\underline{\underline{I}}$ is the identity. This equation was first solved for steady-state flow conditions by Spenley, Cates and McLeish [21] in 1993. The authors predicted that the constitutive relation between shear stress and strain rate would be nonmonotonic in the fast break limit. The non-monotonic behaviour predicted by Eqn. 3.6 is illustrated in Fig. 3.7 ii in blue. Eqn. 3.6 predicts that the viscosity of the fluid will drop below that of the solvent at high strain rates. Because this is physically unreasonable, a solvent viscosity term $\eta_{s}$ is included to provide a lower limit to the fluid's viscosity. This produces a turn-up in the stress $\left(\sigma=\eta_{s} \dot{\gamma}\right)$ at higher strain rates shown in green. 

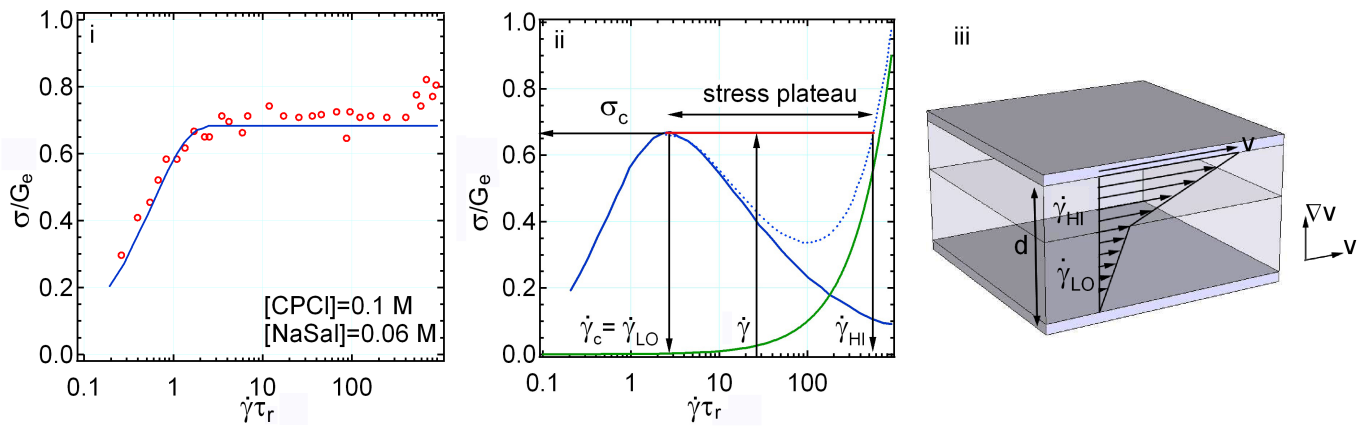

Figure 3.7: i) Reduced experimental flow curve showing a stress plateau disagrees with the ii) non-monotonic flow curve predicted by Eqn. 3.6. The negatively sloping branch of the theoretical flow curve is mechanically unstable. The disagreement between experiment and theory can be reconciled if the fluid spatially partitions into iii) strain rate or shear bands of common stress located on the stable positively sloping lower and upper branchs of the flow curve in simple shear flow conditions. ( $\mathrm{i}$ and ii from [21]).

The mechanical stability of a constitutive relation can be examined using linear stability analysis. In simple shear flow conditions, the one-dimensional Navier-Stokes equation states that $\rho \frac{\partial v_{x}}{\partial t}=\frac{\partial \sigma}{\partial y}$. Taylor-series expanding the constitutive relation gives $\sigma=$ $\sigma_{0}+\frac{\partial \sigma}{\partial \dot{\gamma}} \Delta \dot{\gamma}$. Differentiating with respect to $y$ and combining gives the diffusion equation $\frac{\partial \Delta \dot{\gamma}}{\partial t}=D \frac{\partial^{2} \Delta \dot{\gamma}}{\partial y^{2}}$. If the effective diffusion constant $D=\frac{1}{\rho} \frac{\partial \sigma}{\partial \dot{\gamma}}$ is negative, corresponding to a decreasing stress with increasing strain rate, the simple shear flow is unstable to perturbations in strain rate $[22$.

A non-monotonic constitutive relation is predicted for monodisperse entangled polymer melts/solutions according to the Reptation model. McLeish and Ball considered the effect of this constitutive instability, $\frac{\partial \sigma}{\partial \dot{\gamma}}<0$, on the flow behaviour of monodisperse entangled polymer melts/solution in cylindrical capillary flow in 1986 [23]. In capillary flow, shear stress varies radially according to $\sigma_{r z}=\sigma_{R} r / R$ where $\sigma_{R}$ is the wall stress. They argued that when the stress in the capillary exceeded a critical value, that the strain rate in the fluid would show a discontinuity as the strain rate jumped from the mechanically stable low branch to the mechanically stable high branch, $\frac{\partial \sigma}{\partial \dot{\gamma}}>0$, associated with solvent viscosity, over the unstable region of the flow curve. It was suggested that this discontinuity might account for a spurt effect in which the flow rate increases discontinuously above a critical stress. Following [23] Spenley, Cates and McLeish [21] noted that the declining branch $\frac{\partial \sigma}{\partial \dot{\gamma}}<0$ on the non-monotonic flow curve predicted by Eqn. 3.6 was mechanically unstable and therefore unable to sustain simple shear flow in simple shear flow conditions and predicted that the fluid would subdivide into strain rate bands located at a common stress on the mechanically stable $\frac{\partial \sigma}{\partial \dot{\gamma}}>0$ lower and higher branches. This is illustrated schematically in Fig. 3.7 ii. Here a strain rate $\dot{\gamma}$ is applied to the fluid above critical strain rate $\dot{\gamma}_{c}$. Because of the mechanical instability, the fluid partitions into two or more bands 
with local strain rate $\dot{\gamma}_{L O}$ and $\dot{\gamma}_{H I}$ at a critical stress $\sigma_{c}$. It was asserted that the critical strain rate and critical stress occurred at the local maximum of the non-monotonic flow curve which according to Eqn. 3.6 occurred at a reduced critical strain rate of $\dot{\gamma}_{c}=2.6 / \tau_{r}$ and at a corresponding critical shear stress of $\sigma_{c}=0.67 G_{e}$. An experimental flow curve is compared to the predicted flow curve in Fig. $3.7 \mathrm{i}$. The two show good agreement. It was further predicted that the partitioning should follow the lever rule:

$$
\dot{\gamma}=\phi \dot{\gamma}_{L O}+[1-\phi] \dot{\gamma}_{H I}
$$

where $\phi$ is the volume fraction of low strain rate band. Shear banded flow is illustrated in Fig. 3.7 iii which shows a fluid dividing into two strain rate bands in simple shear flow conditions.

Shortly after this prediction was made, banded structure was observed experimentally in the vicinity of the stress plateau using flow birefringence in [24]. When viewed down the vorticity axis $(\nabla \times \mathbf{v})$ of a cylindrical Couette geometry between crossed polarisers, bands were clearly visible. Because the optical properties of an entangled polymer-like solution depend on anisotropy which itself depends on strain rate, these 'birefringence' bands were interpreted as strain rate bands. Fig. 3.8 ii clearly illustrates birefringence banding observed in a later paper [25]. Note that only two bands are visible and the brighter band, which is assumed to be the high strain rate band, expands out from the inner wall with increasing strain rate. This is to be expected. In the cylindrical-Couette geometry, the shear stress is not constant as is assumed in simple shear flow. Instead it varies radially according to Eqn. 2.27. For $\dot{\gamma}_{L O}<\dot{\gamma}<\dot{\gamma}_{H I}$ the shear stress in the gap will straddle the critical stress. The high strain rate band is therefore expected to form in the high stress region of the geometry near the inner wall with a width given by the lever rule (Eqn. 3.7).

Birefringence measurements provide only indirect evidence. It is assumed that a correlation between optical anisotropy and strain rate exists. The first direct evidence of shear banding came from NMR velocimetry [26]. Shear banding in wormlike micelles has since been observed directly using heterodyne photo correlation spectroscopy (PCS) [27, particle imaging velocimetry (PIV) 28] 29] [30, and NMR [31, 32, 33] 34] 35] 36]. Fig. 3.8 ii shows measurements of the fluid velocity in the flow direction across the gap of Couette geometry using heterodyne PCS. Again the high strain rate band is observed to expand outwards from the inner wall as the apparent strain rate $\dot{\gamma}$ increases. Indirect evidence, where shear banding is inferred from structural anisotropy has been obtained using small angle neutron scattering (SANS) 37] 25] [38] 39], small angle light scattering (SALS) and NMR, and flow birefringence [40] [4] [42] [43] [44].

The prediction and subsequent verification of shear banding in wormlike micelles has led to a search for shear banding in other complex fluids. Shear banding has been observed in colloidal-like suspensions [47, lamellar phase surfactant solutions [48] and nematic liquid crystals. Although some controversial evidence of shear banding in polymer-like entangled DNA solutions exists [49] [50] [50] [51] the consensus appears to be that shear banding has not been definitively observed in entangled polymer solutions or melt under steady-state 


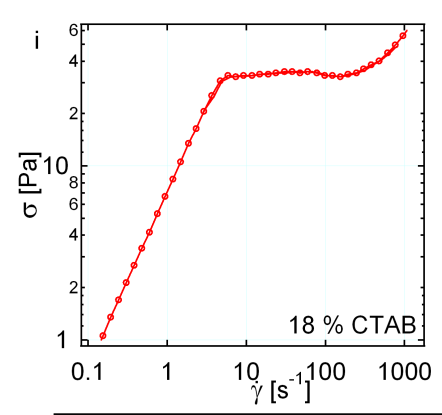

ii
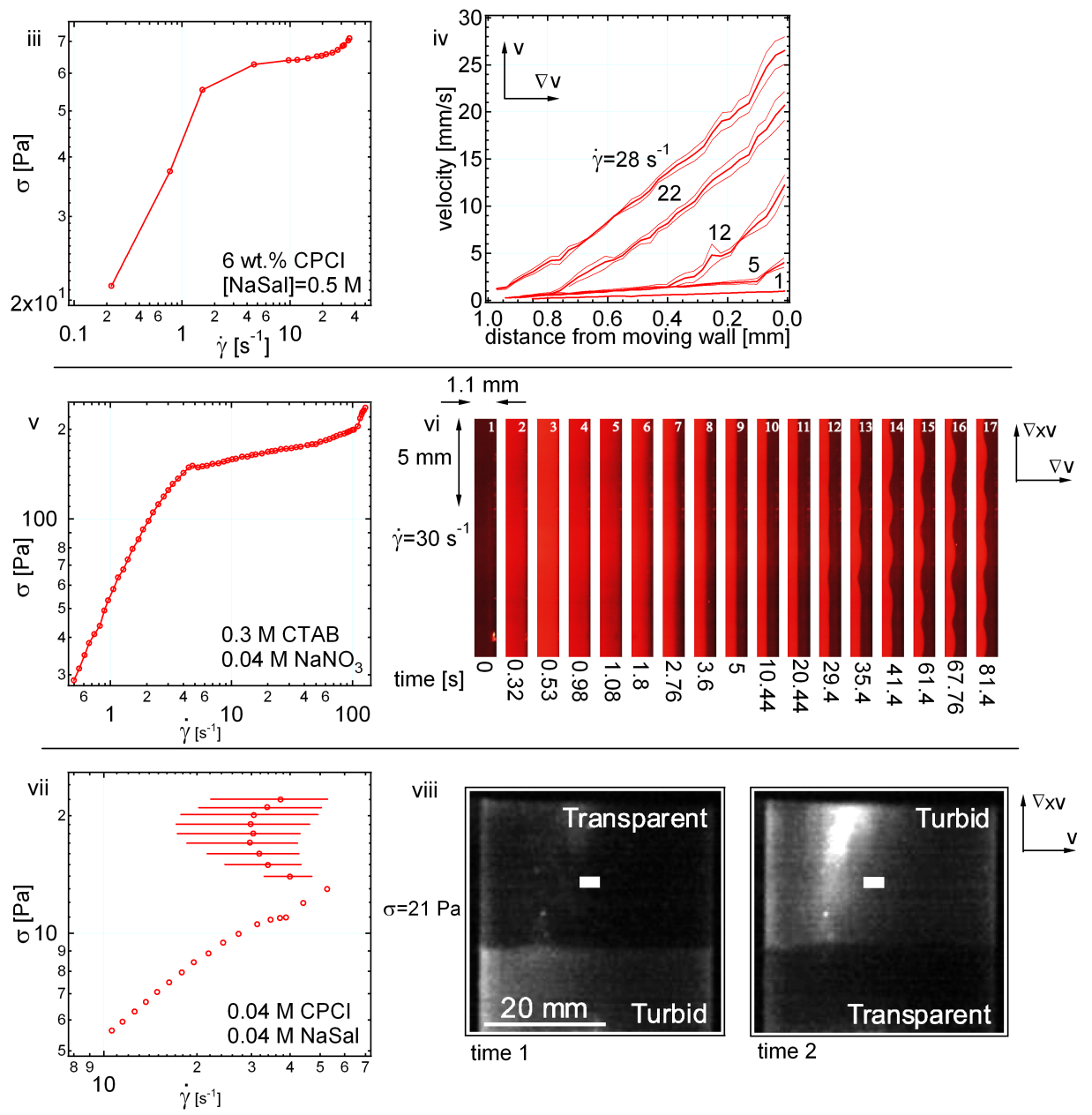

Figure 3.8: Flow curve (left) with optical measurements (right) of selected wormlike micelles solutions. i-ii) Flow birefringence and iii-iv) heterodyne PCS observations of shear banding in wormlike micelles solutions (from [25] and [27]). v-vi) Shear induced turbidity in a shear thinning and vii-viii) a shear thickening wormlike micelles solution (from [45] and [46]). 
flow conditions. This is somewhat surprising given that entangled monodisperse linear polymers solutions/melts are also expected to possess a non-monotonic flow curve. This is potentially a problem for a shear banding model based only on mechanical instability. This issue was addressed by Cates in 2006 where it was postulated that a processes called convected constraint release prevents shear banding in monodisperse polymer solutions.

The flow curve shown in Fig. 3.7 is unstable and multi-valued over a range of stresses. In principle, the critical stress could occur at any stress in this range. Experimentally, it is found to occur at a well defined critical stress. Critical stress selection was investigated theoretically in 2000 [52] using a modified version of the Johnson-Segalman model. The Johnson-Segalman (JS) model can be used to describe the constitutive relation when the polymer strands slip with respect to the solvent. Here the constitutive relation for the polymer is

$$
\underline{\underline{\sigma}}_{p}+\tau \underline{\underline{\underline{D}}}_{p}=2 \mu \underline{\underline{D}}
$$

$\underline{\underline{\sigma}}$ is a time derivative (Gordon-Schowalter convected derivative) which includes dependency on a slip parameter $a$ [5]. Although Eqn. 3.8 has the form of constitutive relation Eqn. 2.14 it is significantly more difficult to solve because of the complexity of the time derivative. Eqn. 3.8 includes only the contribution of the polymer stress to the total stress; the total stress contains a contribution from the solvent

$$
\underline{\underline{\sigma}}=\underline{\underline{\sigma}}_{p}+\underline{\underline{\sigma}}_{s}
$$

Eqn. 3.8 and 3.9 predict that the shear stress is non-monotonic in strain rate:

$$
\sigma=\frac{G \dot{\gamma} \tau}{1+\left(1-a^{2}\right) \dot{\gamma}^{2} \tau^{2}}+\eta_{s} \dot{\gamma}
$$

and represents the simplest tensorial model that gives a flow curve with a negative slope. In [52] Eqn. 3.8 was modified to include a diffusion term

$$
\underline{\underline{\sigma}}_{p}+\tau \underline{\underline{\underline{\sigma}}}_{p}+D \nabla^{2} \underline{\underline{\sigma}}_{p}=2 \mu \underline{\underline{D}}
$$

This has been called the diffusive Johnson-Segalman or dJS model. The diffusion term reflects stress relaxation by diffusion of differently strained strands. By solving Eqn. 3.11 numerically, it was demonstrated in 53 that the inclusion of the diffusion term produced unique and repeatable critical stress. It was subsequently demonstrated that this additional diffusion term could also stabilise the shear bands and broaden the interface between the bands.

\subsubsection{Coupling between flow and concentration}

Changes in the turbidity of complex fluids generally reflect changes in the microstructure. This property is routinely used by physical chemists to map out phase transitions 


\begin{tabular}{|l|l|l|}
\hline surfactant & salt/counterion & reference \\
\hline$[\mathrm{CPCl}]=0.04 \mathrm{M}$ & {$[\mathrm{NaSal}]=0.04 \mathrm{M}$} & {$[46][57][58][59]$} \\
{$[\mathrm{CPCl}]=0.3 \mathrm{M}$} & {$\left[\mathrm{NaNO}_{3}\right]=0.405 \mathrm{M}$} & {$[45]$} \\
{$[\mathrm{CTAB}]=0.003-0.25 \mathrm{wt} . \%$} & {$[\mathrm{NaSal}] /[\mathrm{CTAB}]=1$} & {$[60]$} \\
{$[\mathrm{TTAA}]=0.0075 \mathrm{M}$} & {$[\mathrm{NaSal}]=0.0075 \mathrm{M}$} & {$[61]$} \\
{$[\mathrm{CTAB}]=0.08 \mathrm{M}$} & {$[\mathrm{NaSal}]=0.08 \mathrm{M}$} & {$[62]$} \\
{$[\mathrm{CPCl}]=0.3 \mathrm{M}$} & {$[\mathrm{NaNO}]=1.79 \mathrm{M}$} & {$[63]$} \\
{$[\mathrm{CPCl}]=0.03 \mathrm{M}$} & {$[\mathrm{NaSal}]=0.23 \mathrm{M}$} & {$[64]$} \\
{$[\mathrm{CTAB}]=0.05 \mathrm{M}$} & {$[\mathrm{NaSal}]=0.05-0.2 \mathrm{M}$} & {$[65]$} \\
{$[\mathrm{EHAC}]=0.04 \mathrm{M}$} & {$[\mathrm{NaSal}]$ or $[\mathrm{NaCl}]$} & {$[66]$} \\
\hline
\end{tabular}

Table 3.2: Wormlike micelles solutions reported to exhibit shear induced turbidity (EHAC=erucyl bis(hydroxyethyl)methyl ammonium chloride, TTAA=tallowalkyl ammonium acetate).

in the phase diagram. Some entangled polymer solutions show shear induced turbidity. Historically, this increase in turbidity was interpreted as signaling a shear induced or 'non-equilibrium' phase transition. In 1989, Helfand and Fredrickson proposed instead that this shear induced turbidity might be associated with 'critical concentration fluctuations' [54]. According to the Helfand and Fredrickson model, flow increases a stretched polymer chain's tendency to diffuse from a less entangled region (less restricted, lower viscosity) to a more entangled (more restricted, higher viscosity) region enhancing local concentration fluctuations naturally present in polymer solutions. These concentration fluctuations do not spontaneously grow in size with time as would occur during equilibrium phase separation [55]. Experimental evidence for critical concentration fluctuations in polymer solutions is discussed in [55] [56].

Shear induced turbidity has been observed in many wormlike micelles solutions. An indicative list is given in Table 3.2 . Fig. 3.8 v-viii shows direct observations of shear induced turbidity in two wormlike micelles solutions in the cylindrical-Couette geometry. In Fig. 3.8 vi a turbid (lighter) band is observed to form near the inner wall (from [45]). This behaviour is reminiscent of the shear banding seen in Fig. 3.8 ii and suggests that a correlation between strain rate and turbidity may exist. In Fig. 3.8 viii turbid bands are stacked along the vorticity axis (from [46]). In both Fig. 3.8 vi and 3.8 viiii turbidity varies in space and time.

The relationship between flow-concentration coupling and shear-induced phase separation in complex fluids was considered in 1995 [22]. Using linear stability analysis, the authors predicted that concentration fluctuations like those predicted by HF critical concentration fluctuations could act as a feedback mechanism enhancing the tendency of a mechanically unstable fluid to form bands. These bands were expected to show concentration differences. Stress banding was also predicted for the first time in this paper. In shear (or gradient) banding, the viscosity bifurcates along the gradient velocity direction. In stress (or vorticity) banding, the viscosity bifurcates along the vorticity direction as 

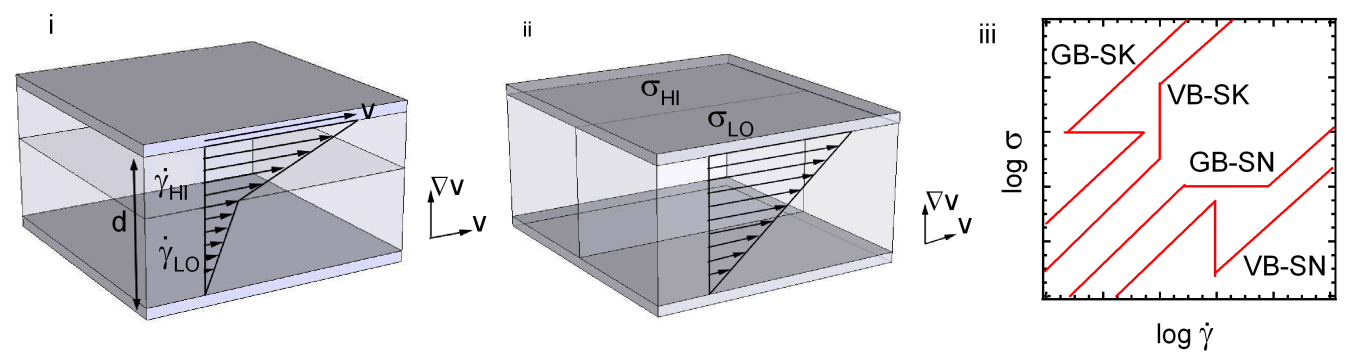

Figure 3.9: i) Gradient (or shear) banding and ii) vorticity (or stress) banding is predicted to occur when concentration is coupled to flow. iii) Universal flow curves for a two phase shear induced transition that results in either gradient banding (GB) or vorticity banding (VB) and shear thinning (SN) or shear thickening (ST) [67].

illustrated in Fig. 3.9 i and ii.

Shear-induced phase transitions in complex fluids were examined in [67]. Here, the universal rheological signatures of a complex fluid that could be "sheared into" a new flow stabilised, non-equilibrium phase that 1) could coexist with the original phase under either constant strain rate (vorticity banding - VB) or constant stress conditions (gradient banding - GB ) and 2) had either a higher (resulting in shear thickening - SK) or lower viscosity (resulting in shear thinning - SN) was developed. The rheological signatures of these fluids is shown in Fig. 3.9 iii. Gradient or vorticity bands coexist in the horizontal or vertical sections of the flow curve. As in 22 it was assumed that this partitioning into bands was associated with a partitioning of concentration. This work was subsequently extended in [68] where it was argued that even a non-conserved parameter such as ordering (as opposed to a conserved parameter such as concentration) could precipitate a banding transition.

In a series of papers in 2003 [69] [70] [71] Fielding and Olmsted extended the dJS model to include coupling to flow enhanced HF concentration fluctuations. They called this extended model dJS $\phi$. From their numerical study it was determined that coupling to concentration would broaden the stress plateau by reducing both critical stress and strain rate. The strain rate bands formed were expected to show concentration differences with the high strain rate band showing a lower concentration. This difference in concentration between the bands was expected to produce a slight upwards slope to the plateau.

\subsubsection{Stress fluctuations and flow instabilities}

The response of a wormlike micelles solution under constant stress or strain rate conditions shows temporal features not seen in entangled polymer solutions/melts including 1) sigmoidal dynamics, 2) rheo-chaos and 3) slip-induced flow instability.

The application of strain rate to a semi-dilute wormlike micelles solution may result 

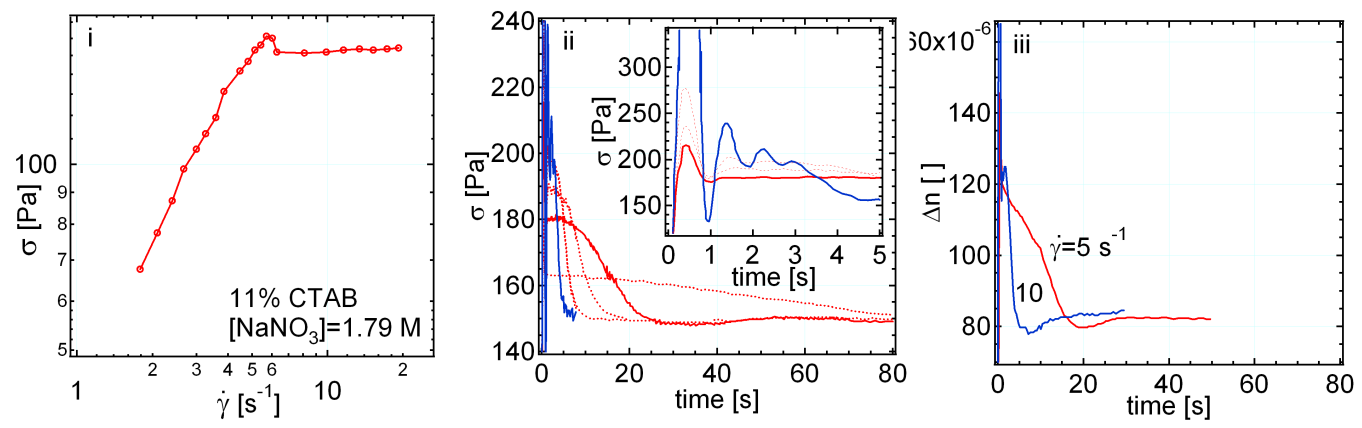

Figure 3.10: i) Flow curve with ii) transient stress showing a 'sigmoidal' stress relaxation after a strain rate step exceeds the critical strain rate. iii) This sigmoidal stress relaxation is correlated with changes in the birefringence $(\Delta n)[63$.

in a damped stress oscillation which periodically exceeds the steady-state value. This behaviour is called a stress overshoot and is observed in entangled polymer/melts and is predicted by Eqn. 2.61. A stress overshoot is shown in Fig. 3.10 ii (inset). For strain rates that exceed the critical stress a slow relaxation of the form [72]

$$
\sigma(t)=\sigma_{c}+\Delta \sigma \exp \left[-\left(t / \tau_{s}\right)^{2}\right]
$$

is observed in addition to the stress overshoot. The characteristic time $\tau_{s}$ of this 'sigmoidal' relaxation is inversely related to the difference between the critical strain rate $\dot{\gamma}_{c}$ and the apparent strain rate $\dot{\gamma}$ and is correlated with changes in the optical birefringence of the material (Fig. 3.10 iii) 63]. In [72] the authors argued that the sigmoidal decay was not readily explained by mechanical instabilities indicating that a non-equilibrium phase transition was occurring. It was subsequently demonstrated that the dJS model was capable of reproducing this behavior [52] 73].

In some semi-dilute wormlike micelles solutions stress may fluctuate chaotically under constant strain rate conditions. This phenomenon has been called rheo-chaos. Chaos is characterised by the extreme sensitivity of a dynamic system to the initial conditions - a perturbation to the initial conditions will result in a response that deviates exponentially from the response of the unperturbed initial case. Chaos can be established by calculating the Lyapanov coefficient of a time-varying signal using time-series analysis. A positive coefficient indicates exponential growth and chaos. Chaos was first identified in steady-state shear stress in [74. Fig. 3.11 ii illustrates chaotic stress fluctuations for the same system from a later paper [75]. These fluctuations are correlated with both the optical intensity of light transmitted down the vorticity axis and the birefringence. This phenomenon was investigated by Fielding and Olmsted in 2003 [76]. Here, Fielding and Olmsted considered the one-dimensional flow of a fluid with a mechanically unstable constitutive relation (which resembled that given by the dJS model) that was coupled to an external parameter with its own dynamics that itself was dependent on strain rate. This external parameter could represent the length of the micelles for example. The resulting coupled PDE were 

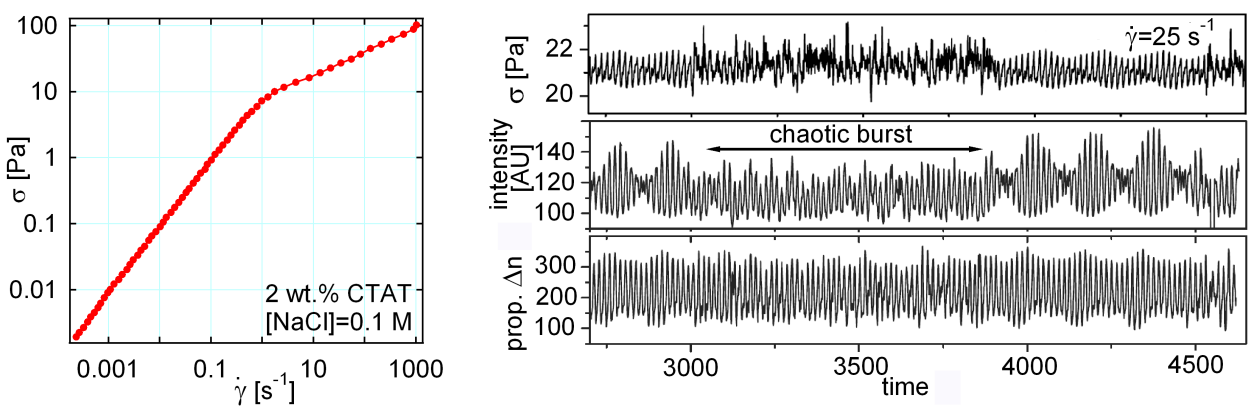

Figure 3.11: i) Flow-curve with ii) transient stress (top), transmissivity (middle) and birefringence (bottom) demonstrating rheo-chaos and the correlation between stress, transmissivity and birefringence in a wormlike micelles solution [75].

solved numerically. Strong coupling was found to destabilise the high strain rate branch of the constitutive relation producing chaotic stress fluctuations in constant strain rate conditions above the critical stress. These stress fluctuations were correlated with fluctuations in the location of the high strain rate band(s) in the gap.

The stability of the strain rate bands calculated according to the dJS model was reexamined by Fielding in 2005 [77]. Using linear analysis, it was determined that well developed one-dimensional strain rate bands were potentially unstable to perturbation in the flow $(\mathbf{v})$ or vorticity $(\nabla \times \mathbf{v})$ direction. This suggested that the dimensionality of the solution of the dJS model might influence its stability. Fielding investigated twodimensional solutions in the $\nabla \mathbf{v}-\mathbf{v}$ plane in 2006 [78] and in the $\nabla \mathbf{v}-\nabla \times \mathbf{v}$ plane in 2007 [79. In [78 perturbations were found to produce undulations at the interface between the bands. In some cases these undulations were found to ripple - this rippling was correlated with oscillation in stress. If multiple bands were present, the stress could fluctuate chaotically. These results indicated that coupling, described above, was not required to produce fluctuations in stress. In [79] Fielding showed that perturbations could develop into slow velocity rolls stacked along the vorticity direction. These rolls were accompanied by undulations in stress along the vorticity axis.

Wall slip, where the fluid fails to adhere to the working surfaces of the rheometry geometry, has been correlated with flow instabilities in some wormlike micelles solutions. This is illustrated in Fig. 3.12 (from [80]). Velocity in the flow direction is shown using greyscale in Fig. 3.12 ii at a constant apparent strain rate $\dot{\gamma}$. Clearly the velocity is oscillating in time. The velocity of the fluid at both the inner and outer wall is shown in Fig. 3.12 iii. Although the velocity of the moving inner wall of the cylindrical-Couette is fixed, the velocity of the fluid at the inner wall shows significant oscillations with time indicating that slip is occurring. Slip/flow instabilities are correlated with changes in the birefringence but are not correlated with measured stress as is shown in Fig. 3.12 iii. 

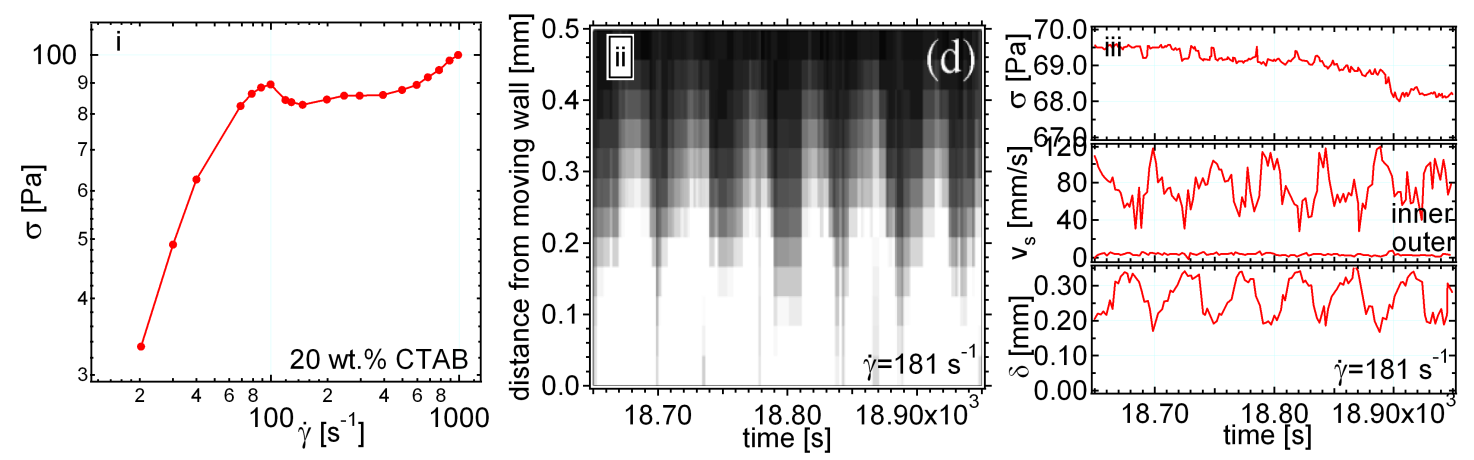

Figure 3.12: i) Flow curve with ii) time resolved velocimetry across the gap of a cylindrical-Couette geometry (lighter grey regions correspond to higher velocities) under constant strain rate conditions demonstrating a correlation between slip at the inner wall, iii)(middle) flow instabilities and iii)(bottom) and birefringence [80]. 


\section{Chapter 4}

\section{Rheo-optics}

\subsection{Introduction}

A mechanical rheometer reports spatially averaged or apparent stresses and strain rates. This spatial averaging can obscure the true flow behaviour of complex fluids that undergo some kind of spatial partitioning like that seen in wormlike micelles solutions. Furthermore, a mechanical rheometer is subject to mechanical limitations that restrict its operating range. To address these issues, the rheology of a complex fluid can be studied using other methods. The study of the rheology of complex fluids using light is called rheooptics. Although almost any optical technique can be used to study complex fluids, the most common optical techniques used are based around ellipsometry and static/dynamic light scattering. Ellipsometry and dynamic light scattering are the principle optical techniques used in this thesis to study wormlike micelles solutions.

Fundamentally, the optical properties of any material depend on the atomic polarisability $\underline{\underline{\alpha}}$ of the constitutive atoms. Electromagnetic radiation accelerates an atom's electrons which reradiate the incident light. The tensor $\underline{\underline{\alpha}}$ describes the relationship between incident and outgoing electromagnetic radiation. If the atomic polarisability is spatially uniform then the medium can be treated as homogeneous - ellipsometry can be useful if the medium is homogeneous. If the atomic polarisability is not uniform as is often the case with complex fluids, the medium is optically inhomogeneous - light scattering may be more useful.

In this chapter, the plane wave and Green function solutions to the Maxwell equations are introduced. Plane wave solutions are used to understand the propagation of light in optically homogeneous media. The Green function solutions are used to understand propagation or scattering in optically inhomogeneous media. The statistics of this scattered light are considered in two cases: the single scattering regime and the highly multiple scattering diffusion approximation. Finally, several rheo-optical properties of polymer

solutions are reviewed. These properties will be useful for understanding observations in 
later chapters.

\subsection{Solving Maxwell equations}

\subsubsection{Maxwell equations}

Light is electromagnetic radiation. The spatiotemporal relationship between the electric $\mathbf{E}$ and magnetic field $\mathbf{B}$ in linear media with permeability $\mu$ and permittivity $\varepsilon$ is described by Maxwell equations. In the absence of free current, charge and optical rotation, Maxwell equations are [81] 82] 12]:

$$
\begin{aligned}
\nabla \cdot \mathbf{E} & =0, \\
\nabla \cdot \mathbf{B} & =0, \\
\nabla \times \mathbf{E} & =-\frac{\partial \mathbf{B}}{\partial \mathbf{t}}, \\
\nabla \times \mathbf{B} & =\mu \varepsilon \frac{\partial \mathbf{E}}{\partial t} .
\end{aligned}
$$

The behaviour of light inside a linear dielectric can be determined by solving Eqn. 4.1 4.4 for $\mathbf{E}$ with the appropriate boundary conditions.

\subsubsection{Plane wave solution}

Eqn. 4.34 .4 can be combined to decouple magnetic and electric fields

$$
\underline{\underline{\varepsilon}} \mu \frac{\partial^{2} \mathbf{E}}{\partial t^{2}}+\nabla \times \nabla \times \mathbf{E}=0
$$

Because $\nabla \cdot \mathbf{E}=0$

$$
\underline{\underline{\varepsilon}} \mu \frac{\partial^{2} \mathbf{E}}{\partial t^{2}}-\nabla^{2} \mathbf{E}=0
$$

Eqn. 4.6 is the wave equation and admits plane wave solutions of the form

$$
\mathbf{E}(\mathbf{r}, t)=\exp [i(\mathbf{k} \cdot \mathbf{r}-\omega t+\delta)],
$$

which propagate with a velocity $v=1 / \sqrt{\mu \varepsilon}$. This velocity is slower than free space velocity of propagation $c$ by a factor $n$ called the refractive index

$$
n=\frac{c}{v}=\sqrt{\frac{\mu_{0} \varepsilon_{0}}{\mu \varepsilon}} .
$$

For magnetically inert media $\mu=\mu_{0}$ and $n^{2}=\varepsilon / \varepsilon_{0}=\epsilon$ where $\epsilon$ is the dielectric constant. In terms of $n,|\mathbf{k}|=k=2 \pi n / \lambda$ where $\lambda$ is the free space wavelength. The dielectric 
constant of the medium can be related to the average atomic polarisability $\bar{\alpha}$ of the medium by the Lorentz-Lorenz relation 81 ]

$$
\frac{\epsilon-1}{\epsilon+2}=\frac{n^{2}-1}{n^{2}+2}=N \bar{\alpha}
$$

where $N$ is the number density. In general $\varepsilon, \epsilon, n$ and $\alpha$ are second rank tensors. The plane wave solution details depend on the form of the dielectric constant tensor.

\subsubsection{Green function solution}

A Green function $G(\mathbf{R}, \mathbf{r})$ is a solution to an inhomogeneous differential equation of the form $L G(\mathbf{R}, \mathbf{r})=\delta(\mathbf{R}-\mathbf{r})$ where $L$ is a linear differential operator. If $G(\mathbf{R}, \mathbf{r})$ can be determined then this solution can be used to construct a solution to the more general equation $L \phi(\mathbf{R})=f(\mathbf{R})$ by integration $\phi(\mathbf{R})=\int f(\mathbf{r}) G(\mathbf{R}, \mathbf{r}) d \mathbf{r}$ [83]. In inhomogeneous media $\epsilon$ is a function of position. Assuming $\mathbf{E}(t) \propto \exp [i \omega t]$ and noting that $\left(\mu_{0} \varepsilon \omega\right)^{2}=$ $k^{2} m^{2}$ where $m$ is the ratio of the refractive index of the scattering center $n_{p}$ to the host medium $m=n_{p} / n$ Eqn. 4.5 can be rewritten [12.

$$
\boldsymbol{\nabla} \times \boldsymbol{\nabla} \times \mathbf{E}(\mathbf{R})-k^{2} \mathbf{E}(\mathbf{R})=k^{2}\left[\underline{\underline{m}}^{2}(\mathbf{R})-\underline{\underline{I}}\right] \cdot \mathbf{E}(\mathbf{R}) .
$$

The required Green function solution solves [12]

$$
\nabla \times \nabla \times \underline{\underline{G}}(\mathbf{R}, \mathbf{r})-k^{2} \underline{\underline{G}}(\mathbf{R}, \mathbf{r})=\underline{\underline{I}} \delta(\mathbf{R}-\mathbf{r}) .
$$

Note that because the Green function is a tensor the point source is a quadrupole. If $|\mathbf{R}| \gg$ $|\mathbf{r}|$ corresponding to the distant detection at $\mathbf{R}$ of a small source at $\mathbf{r}$ (Born approximation) then the Green function is approximately [12]

$$
\underline{\underline{G}}(\mathbf{R}, \mathbf{r})=[\underline{\underline{I}}-\hat{\mathbf{R}} \hat{\mathbf{R}}] \frac{\exp [i k R]}{4 \pi R} \exp \left[-i \mathbf{k}_{f} \cdot \mathbf{r}\right]
$$

where $\mathbf{k}_{f}=k \hat{\mathbf{R}}$. The field in this approximation is then [12]

$$
\begin{aligned}
\mathbf{E}(\mathbf{R}, t) & =\mathbf{E}_{u}(\mathbf{r}, t)+\mathbf{E}_{s}(\mathbf{r}, t) \\
& =\mathbf{E}_{u}(\mathbf{r}, t)+\frac{\exp [i k R]}{4 \pi R} k_{f}^{2} \int \exp \left[-i \mathbf{k}_{f} \cdot \mathbf{r}\right][\underline{\underline{I}}-\hat{\mathbf{R}} \hat{\mathbf{R}}] \cdot\left[\underline{\underline{m}}^{2}(\mathbf{r})-\underline{\underline{I}}\right] \cdot \mathbf{E}_{0}(\mathbf{r}, t) d \mathbf{r}
\end{aligned}
$$

$\mathbf{E}_{u}(\mathbf{r}, t)$ is the unscattered component, $\mathbf{E}_{s}(\mathbf{r}, t)$ is the scattered field and $\mathbf{E}_{0}(\mathbf{r}, t)$ is the incident field [11]. Note that Eqn. 4.14 implies that light is scattered by variations in the refractive index tensor $\underline{\underline{m}}^{2}(\mathbf{r})-\underline{\underline{I}} \neq 0$. As before, the solution details depend on the form of the (relative) refractive index/dielectric field tensor. 


\subsection{Propagation in homogeneous media}

\subsubsection{Propagation in isotropic materials}

In homogeneous optically isotropic media, the permittivity $\varepsilon$ is a scalar quantity. The plane wave solution in this medium can be determined by substituting Eqn. 4.7 into Eqn. 4.6 to give

$$
\mathbf{k} \times \mathbf{k} \times \mathbf{E}=\omega^{2} \mu_{0} \varepsilon \mathbf{E} .
$$

This equation admits two linearly independent solutions of the form:

$$
\begin{aligned}
& \mathbf{E}_{1}(\mathbf{r}, t)=E_{0 n} \exp \left[i\left(\mathbf{k} \cdot \mathbf{r}-\omega t-\delta_{n}\right)\right] \hat{\mathbf{n}}, \\
& \mathbf{E}_{2}(\mathbf{r}, t)=E_{0 m} \exp \left[i\left(\mathbf{k} \cdot \mathbf{r}-\omega t-\delta_{m}\right)\right] \hat{\mathbf{m}},
\end{aligned}
$$

where $\hat{\mathbf{n}}$ and $\hat{\mathbf{m}}$ are conventional unit vectors such that $\mathbf{k} \cdot \hat{\mathbf{n}}=0, \mathbf{k} \cdot \hat{\mathbf{m}}=0$ and $\hat{\mathbf{n}} \times \hat{\mathbf{m}}=0$. $\hat{\mathbf{n}}$ and $\hat{\mathbf{m}}$ fix the direction of the field. By superposition the general solution is

$$
\mathbf{E}(\mathbf{r}, t)=\mathbf{E}_{1}(\mathbf{r}, t)+\mathbf{E}_{2}(\mathbf{r}, t) .
$$

Because the direction of the field is fixed, each solution is said to be linearly polarised. The superposition of the two fields may also be linearly polarised (if $\delta_{n}=\delta_{m}$ ) or circularly polarised, where the field direction circulates about the direction of propagation (when $E_{0 n}=E_{0 m}$ and $\delta_{n}=i \delta_{m}$ ) but will generally be elliptically polarised, where the locus of the field follows an ellipse about the direction of propagation (when $E_{0 n} \neq E_{0 m} \neq 0$ and $\left.\delta_{n} \neq \delta_{m}\right)$.

\subsubsection{Propagation in anisotropic material}

In homogeneous optically anisotropic media, the permittivity $\underline{\underline{\varepsilon}}$ is a tensor quantity. Following Klein and Furtak [84, the plane wave solution in this medium can be determined by substituting Eqn. 4.7 into Eqn. 4.6

$$
\mathbf{k} \times \mathbf{k} \times \mathbf{E}=\omega^{2} \mu_{0} \underline{\underline{\varepsilon}} \cdot \mathbf{E},
$$

which implies that

$$
\left(\left[\begin{array}{ccc}
-k_{y}^{2}-k_{z}^{2} & k_{x} k_{y} & k_{x} k_{z} \\
k_{y} k_{x} & -k_{x}^{2}-k_{z}^{2} & k_{y} k_{z} \\
k_{z} k_{x} & k_{z} k_{y} & -k_{x}^{2}-k_{y}^{2}
\end{array}\right]+\mu_{0} \omega^{2}\left[\begin{array}{ccc}
\varepsilon_{x x} & \varepsilon_{x y} & \varepsilon_{x z} \\
\varepsilon_{y x} & \varepsilon_{y y} & \varepsilon_{y z} \\
\varepsilon_{x z} & \varepsilon_{y z} & \varepsilon_{z z}
\end{array}\right]\right) \cdot \mathbf{E}=0 .
$$

$\underline{\underline{\varepsilon}}$ is Hermitian and can be diagonalised by the rotation transform. In this rotated coordinate system, the permittivity tensor can have one of three forms:

$$
\underline{\underline{\varepsilon}}_{\text {isotropic }}=\left[\begin{array}{ccc}
\varepsilon_{x} & 0 & 0 \\
0 & \varepsilon_{x} & 0 \\
0 & 0 & \varepsilon_{x}
\end{array}\right], \quad \underline{\underline{\varepsilon}}_{\text {uniaxial }}=\left[\begin{array}{ccc}
\varepsilon_{x} & 0 & 0 \\
0 & \varepsilon_{y} & 0 \\
0 & 0 & \varepsilon_{y}
\end{array}\right], \quad \underline{\underline{\varepsilon}}_{\text {biaxial }}=\left[\begin{array}{ccc}
\varepsilon_{x} & 0 & 0 \\
0 & \varepsilon_{y} & 0 \\
0 & 0 & \varepsilon_{z}
\end{array}\right] .
$$


The orthogonal unit vectors in this rotated coordinate system correspond to the principle directions of the permittivity/dielectric/refractive index tensor.

Note that the dielectric constant or refractive index may be complex $n \rightarrow n^{\prime}+i n^{\prime \prime}$. The imaginary part results in attenuation. Uniaxial or biaxial materials are said to be birefringent if the real part of the principle refractive indices differs or dichroic if the imaginary parts of the refractive indices show differences.

Assuming that the anisotropic medium is oriented so that its principle direction is aligned with the lab frame, that the direction of propagation is $\boldsymbol{\kappa}=\frac{k_{x}}{k} \hat{\mathbf{i}}+\frac{k_{y}}{k} \hat{\mathbf{j}}+\frac{k_{z}}{k} \hat{\mathbf{k}}=$ $\kappa_{x} \hat{\mathbf{i}}+\kappa_{y} \hat{\mathbf{j}}+\kappa_{z} \hat{\mathbf{k}}$ and that the principle refractive indices are $n_{x}^{2}=\varepsilon_{x} / \varepsilon_{0}, n_{y}^{2}=\varepsilon_{y} / \varepsilon_{0}$, $n_{z}^{2}=\varepsilon_{z} / \varepsilon_{0}$, Eqn. 4.20 can be rewritten

$$
\left(n^{2}\left[\begin{array}{ccc}
\kappa_{x}^{2}-1 & \kappa_{x} \kappa_{y} & \kappa_{x} \kappa_{z} \\
\kappa_{y} \kappa_{x} & \kappa_{y}^{2}-1 & \kappa_{y} \kappa_{z} \\
\kappa_{z} k_{x} & \kappa_{z} \kappa_{y} & \kappa_{z}^{2}-1
\end{array}\right]+\left[\begin{array}{ccc}
n_{x}^{2} & 0 & 0 \\
0 & n_{y}^{2} & 0 \\
0 & 0 & n_{z}^{2}
\end{array}\right]\right) \cdot \mathbf{E}=0
$$

This equation has the non-trivial solution

$$
\frac{1}{n^{2}}=\frac{\kappa_{x}}{n^{2}-n_{x}^{2}}+\frac{\kappa_{y}}{n^{2}-n_{y}^{2}}+\frac{\kappa_{z}}{n^{2}-n_{z}^{2}} .
$$

For example, if the direction of propagation is $\boldsymbol{\kappa}=\kappa_{x} \hat{\mathbf{i}}+\kappa_{z} \hat{\mathbf{k}}=\sin \theta \hat{\mathbf{i}}+\cos \theta \hat{\mathbf{k}}$, through a biaxial material oriented with principle axis aligned with the lab frame:

$$
\begin{aligned}
{\left[n^{2} \kappa_{z}^{2}-n_{x}^{2}\right] E_{x}-\left[n^{2} \kappa_{x} \kappa_{z}\right] E_{z} } & =0 \\
{\left[n^{2}-n_{y}^{2}\right] E_{y} } & =0 \\
{\left[n^{2} \kappa_{x} \kappa_{z}\right] E_{x}+\left[n^{2} \kappa_{x}^{2}-n_{z}^{2}\right] E_{z} } & =0
\end{aligned}
$$

The two independent plane wave solutions to Eqn. 4.23 4.25 are:

$$
\begin{array}{r}
E_{1}(\mathbf{r}, t)=E_{0 y} \exp \left[i\left(\frac{2 \pi n_{y}}{\lambda} \boldsymbol{\kappa} \cdot \mathbf{r}-\omega t-\delta_{y}\right)\right] \hat{\mathbf{j}}, \quad\left(E_{x}=0, E_{z}=0, E_{y} \neq 0\right), \\
E_{2}(\mathbf{r}, t)=E_{0} \exp \left[i\left(\frac{2 \pi n_{a}}{\lambda} \boldsymbol{\kappa} \cdot \mathbf{r}-\omega t-\delta\right)\right] \hat{\mathbf{i}}+E_{0} \exp \left[i\left(\frac{2 \pi n_{b}}{\lambda} \boldsymbol{\kappa} \cdot \mathbf{r}-\omega t-\delta\right)\right] \hat{\mathbf{k}}, \\
\left(E_{x} \neq 0, E_{z} \neq 0, E_{y}=0\right),
\end{array}
$$

where $n_{a}^{2}=\frac{n_{x}^{4} \kappa_{x}^{2}}{\kappa_{x}^{2} n_{x}^{2}+\kappa_{x}^{2} n_{z}^{2}}$ and $n_{b}^{2}=\frac{n_{z}^{2} n_{x}^{2} \kappa_{x} \kappa_{z}}{\kappa_{x}^{2} n_{x}^{2}+\kappa_{x}^{2} n_{z}^{2}}$. Clearly if $\theta=0, \boldsymbol{\kappa}=\kappa_{z} \hat{\mathbf{k}}$ and

$$
\begin{aligned}
E(\mathbf{r}, t) & =E_{2}(\mathbf{r}, t)+E_{1}(\mathbf{r}, t) \\
& =E_{0 x} \exp \left[i\left(\frac{2 \pi n_{x}}{\lambda} z-\omega t-\delta_{x}\right)\right] \hat{\mathbf{i}}+E_{0 y} \exp \left[i\left(\frac{2 \pi n_{y}}{\lambda} z-\omega t-\delta_{y}\right)\right] \hat{\mathbf{j}}
\end{aligned}
$$

Transmission through an anisotropic medium will generally change the polarisation state of the incident light. Measurement of the change in polarisation state that occurs on 


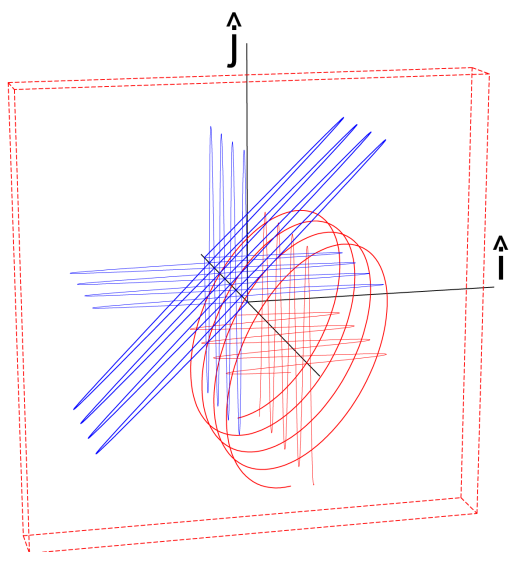

Figure 4.1: Transmission through an anisotropic medium may modify the incident light's polarisation state. The curves in the figure represent the amplitude of the field at an instant. Here, linearly polarised light (blue) is converted into elliptically polarised light (red) on transmission. In ellipsometry, this change in polarisation state is measured.

transmission (or on reflection or due to scattering) is called ellipsometry. When ellipsometry is used to measure the optical anisotropy of complex fluids it is often called 'flow-birefringence'.

\subsubsection{Matrix formulations}

A linear superposition of the solution to Maxwell equations are also solutions

$$
E(\mathbf{r}, t)=\tilde{C}_{1} E_{1}(\mathbf{r}, t)+\tilde{C}_{2} E_{2}(\mathbf{r}, t)
$$

The complex amplitude $\tilde{C}_{1}$ and $\tilde{C}_{2}$ of an actual solution will depend on the 'basis' solutions or polarisations chosen. It is often convenient to work with basis polarisations:

$$
\begin{aligned}
& \mathbf{E}_{x}=\exp [i(k z-\omega t)] \hat{\mathbf{i}}, \\
& \mathbf{E}_{y}=\exp [i(k z-\omega t)] \hat{\mathbf{j}},
\end{aligned}
$$

so that $E(\mathbf{r}, t)=\tilde{A}_{x} \mathbf{E}_{x}+\tilde{A}_{y} \mathbf{E}_{y}$. With this basis solution, the solution space can be represented as a column vector called a Jones vector [85]

$$
\mathbf{J}=\left[\begin{array}{c}
\tilde{A}_{x} \\
\tilde{A}_{y}
\end{array}\right]=\left[\begin{array}{c}
A_{x} \exp \left(i \delta_{x}\right) \\
A_{y} \exp \left(i \delta_{y}\right)
\end{array}\right]
$$

Note directions $\hat{\mathbf{i}}$ and $\hat{\mathbf{j}}$ are defined with respect to the incident $\mathbf{k}_{i}$ and outgoing $\mathbf{k}_{f}$ wave vector and some reference plane (typically the plane containing $\mathbf{k}_{i}$ and $\mathbf{k}_{f}$ ). If the light is 
completely polarised, the polarisation state can be described in terms of the ratio of the two polarisation states

$$
\tilde{r}=\frac{\tilde{A}_{x}}{\tilde{A}_{y}}=\frac{A_{x}}{A_{y}} \exp \left[i\left(\delta_{x}-\delta_{y}\right)\right]=\rho \exp (i \delta)=\tan \Psi \exp (i \delta),
$$

where $\rho=\tan \Psi$ is a commonly used convention.

Reflection and transmission may modify both the phase and amplitude of the field. In linear media, the modified field is a linear superposition of the incident field:

$$
\begin{aligned}
{\left[\begin{array}{l}
A_{x}^{\prime} \exp \left(i \delta_{x}^{\prime}\right) \\
A_{y}^{\prime} \exp \left(i \delta_{y}^{\prime}\right)
\end{array}\right] } & =\left[\begin{array}{ll}
T_{11} & T_{21} \\
T_{12} & T_{22}
\end{array}\right]\left[\begin{array}{c}
A_{x} \exp \left(i \delta_{x}\right) \\
A_{y} \exp \left(i \delta_{y}\right)
\end{array}\right], \\
\mathbf{J}^{\prime} & =\mathrm{T}_{J} \mathbf{J} .
\end{aligned}
$$

The linear transformation $\mathrm{T}_{J}$ is called a Jones matrix. For example, by inspection of Eqn. 4.29 , the Jones Matrix corresponding to the transmission through the biaxial material of thickness $d$ along a principle direction with $\mathbf{E}_{x}$ and $\mathbf{E}_{y}$ aligned with the remaining principle axes is

$$
\mathrm{T}_{J}=\left[\begin{array}{cc}
\exp \left[i 2 \pi\left(n_{x}^{\prime}+i n_{x}^{\prime \prime}\right) d / \lambda\right] & 0 \\
0 & \exp \left[i 2 \pi\left(n_{y}^{\prime}+i n_{y}^{\prime \prime}\right) d / \lambda\right]
\end{array}\right]
$$

The effect of a rotation of the biaxial material about the principle direction $\hat{\mathbf{k}}$ can be effected by applying a rotation transformation $\mathrm{T}_{J}^{\prime}(\phi)=T(\phi) \mathrm{T}_{J} \mathrm{~T}(\phi)^{-1}$.

The Jones calculus is a field representation and is used to describe the polarisation state of light. The Stokes vector/Mueller matrix calculus is an intensity representation of the polarisation state of light. The Stokes vector $\mathbf{I}$ is a $1 \times 4$ column vector and the Mueller matrices $\mathrm{T}_{M}$ are $4 \times 4$ matrices which transform the polarisation state represented by the Stokes vector by matrix multiplication. The Stokes vector and Mueller matrix have the form [85]

$\mathbf{I}=\left[\begin{array}{c}\left\langle\tilde{A}_{x}^{*} \tilde{A}_{x}+\tilde{A}_{y}^{*} \tilde{A}_{y}\right\rangle \\ \left\langle\tilde{A}_{x}^{*} \tilde{A}_{x}-\tilde{A}_{y}^{*} \tilde{A}_{y}\right\rangle \\ \left\langle 2 \operatorname{Re}\left[\tilde{A}_{x}^{*} \tilde{A}_{y}\right]\right\rangle \\ \left\langle 2 \operatorname{Im}\left[\tilde{A}_{x}^{*} \tilde{A}_{y}\right]\right\rangle\end{array}\right], \mathrm{T}_{M}=\left[\begin{array}{cccc}\frac{m_{1}+m_{2}+m_{3}+m_{4}}{2} & \frac{m_{1}-m_{2}-m_{3}+m_{4}}{2} & s_{13}+s_{42} & -d_{13}-d_{42} \\ \frac{m_{1}-m_{2}+m_{3}-m_{4}}{2} & \frac{m_{1}+m_{2}-m_{3}-m_{4}}{2} & s_{13}+s_{42} & d_{42}-d_{13} \\ s_{14}+s_{32} & s_{14}-s_{32} & s_{12}+s_{34} & d_{34}-d_{12} \\ d_{14}+d_{32} & d_{14}-d_{32} & d_{12}-d_{34} & s_{12}-s_{34}\end{array}\right]$.

The matrix element of the Mueller matrix can be derived from the Jones matrix if the element does not depolarise the light: $m_{i}=j_{i} j_{i}^{*}, s_{i k}=\operatorname{Re}\left(j_{i} j_{k}^{*}\right), d_{i k}=\operatorname{Im}\left(j_{i}^{*} j_{k}\right)$ and $j_{1}, j_{2}, j_{3}, j_{4}=T_{11}, T_{22}, T_{12}, T_{21}$. The component $I_{1}$ is the observed intensity. The other components of the Stokes vector can be mapped into the observable $I_{1}$ by multiplication with an appropriate Mueller matrix, which physically corresponds to the introduction of an appropriately orientated polarising optic. If the light is completely polarised $I_{1}^{2}=$ $I_{2}^{2}+I_{3}^{2}+I_{4}^{2}$. If the light is only partially polarised $I_{1}^{2}>I_{2}^{2}+I_{3}^{2}+I_{4}^{2}$. The major advantage of the intensity calculus is that it can be used to describe partial polarisation whereas the field calculus cannot. 


\subsection{Propagation in inhomogeneous media}

\subsubsection{Single scattering regime}

In the Rayleigh approximation, it is assumed that the incident field induces a dipole so that [12]

$$
\left[\underline{\underline{m}}^{2}(\mathbf{r})-\underline{\underline{I}}\right] \cdot \mathbf{E}_{0}(\mathbf{r}, t)=\mathbf{p}(t) \delta\left(\mathbf{r}-\mathbf{r}_{0}(t)\right)
$$

in vacuo. The scattered field in the Born approximation is

$$
\begin{aligned}
\mathbf{E}_{s}(t) & =E_{0} k^{2} \frac{\exp [i k R]}{4 \pi R}[\underline{\underline{I}}-\hat{\mathbf{R}} \hat{\mathbf{R}}] \cdot \mathbf{p}(t) \exp \left[-i \mathbf{k}_{f} \cdot \mathbf{r}_{0}(t)\right] \\
& =\frac{\exp [i k R]}{4 \pi R} \mathbf{k}_{f} \times\left(\mathbf{k}_{f} \times \mathbf{p}(t)\right) \exp \left[-i \mathbf{k}_{f} \cdot \mathbf{r}_{0}(t)\right]
\end{aligned}
$$

The dipole induced by a plane wave linearly polarised in the $\mathbf{n}_{i}$ direction is

$$
\mathbf{p}(t)=\underline{\underline{\alpha}} \cdot \mathbf{E}_{i}=\underline{\underline{\alpha}} \cdot E_{0}\left[i\left(\mathbf{k}_{i} \cdot \mathbf{r}_{0}(t)-\omega t\right)\right] \mathbf{n}_{i},
$$

where $\mathbf{k}_{i}=k \mathbf{n}_{i}$ leads to a scattered field

$$
\mathbf{E}_{s}(t)=E_{0} \frac{\exp [i(k R-\omega t)]}{4 \pi R} \mathbf{k}_{f} \times\left(\mathbf{k}_{f} \times \underline{\underline{\alpha}} \cdot \mathbf{n}_{i}\right) \exp \left[-i \mathbf{q} \cdot \mathbf{r}_{0}(t)\right] .
$$

Here $\mathbf{q}=\mathbf{k}_{f}-\mathbf{k}_{i}$ is called the scattering vector. With an analyser present which selects only a component linearly polarised in the $\mathbf{n}_{f}$ direction the radiated field is

$$
E(t)=\mathbf{n}_{f} \cdot \mathbf{E}_{s}(, t)=E_{0} \frac{\exp [i(k R-\omega t)]}{4 \pi R} k_{f}^{2} \alpha_{i f}(t) \exp \left[-i \mathbf{q} \cdot \mathbf{r}_{0}(t)\right]
$$

where $\alpha_{i f}=\mathbf{n}_{f} \cdot \underline{\underline{\alpha}} \cdot \mathbf{n}_{i}$. For an ensemble of $N$ dipoles with isotropic polarisability [11]

$$
\begin{aligned}
E(t) & =E_{0} \frac{\exp [i(k R-\omega t)]}{4 \pi R} k_{f}^{2} \sum_{n=1}^{N} \alpha_{i f}^{n} \exp \left[-i \mathbf{q} \cdot \mathbf{r}_{n}(t)\right] \\
& =E_{0} \frac{\exp [i(k R-\omega t)]}{4 \pi R} k_{f}^{2} \int \rho(\mathbf{r}, t) \exp [-i \mathbf{q} \cdot \mathbf{r}],
\end{aligned}
$$

where $\rho(\mathbf{r}, t)=\sum \alpha_{i f}^{n} \delta\left(\mathbf{r}-\mathbf{r}_{n}(t)\right)$. If the dipoles are identical, the field $E(t)$ is proportional to the spatial Fourier transform of the dipole density.

In static light scattering, a time averaged intensity $\langle I\rangle=\left\langle E(t) E^{*}(t)\right\rangle$ is measured

$$
\begin{aligned}
\left\langle E(t) E^{*}(t)\right\rangle & =\frac{E_{0}^{2} k^{4}}{16 \pi R^{2}}\left\langle\sum_{m, n} \exp \left[-i \mathbf{q} \cdot\left(\mathbf{r}_{n}(t)-\mathbf{r}_{m}(t)\right)\right]\right\rangle \\
& =\frac{E_{0}^{2} k^{4}}{16 \pi R^{2}} \int\langle\rho(0) \rho(\mathbf{r})\rangle \exp [-i \mathbf{q} \cdot \mathbf{r}] d \mathbf{r} \\
& =\frac{E_{0}^{2} k^{4}}{16 \pi^{2} R^{2}} S(\mathbf{q}) .
\end{aligned}
$$


Here it is assumed that $k_{f}^{2}=k^{2}$. If the dipoles are identical $\langle\rho(0) \rho(\mathbf{r})\rangle$ is the dipole-dipole density autocorrelation function. $S(\mathbf{q})$ is called the static structure factor [86].

In dynamic light scattering, the time dependence of the field correlation $\left\langle E^{*}(0) E(t)\right\rangle$ is measured through an intensity autocorrelation $g_{2}(t)$ [1]

$$
\begin{aligned}
g_{2}(t)=\frac{\langle I(0) I(t)\rangle}{\langle I\rangle^{2}} & =\frac{\left\langle E(0) E^{*}(0) E(t) E^{*}(t)\right\rangle}{\left\langle E E^{*}\right\rangle^{2}} \\
& =\frac{\left\langle E E^{*}\right\rangle^{2}}{\left\langle E E^{*}\right\rangle^{2}}+\frac{\left\langle E(0) E^{*}(t)\right\rangle^{2}}{\left\langle E E^{*}\right\rangle^{2}} \\
& =1+\left|g_{1}(t)\right|^{2} .
\end{aligned}
$$

$g_{1}(t)$ is called the normalised field correlation function. The partitioning in Eqn. 4.50 is only appropriate if the number of scattering centres is significant and their positions are statistically independent - these conditions are "probably satisfied by the vast majority of applications" [1]. From Eqn. 4.45] it is seen that

$$
\begin{aligned}
g_{1}(t) & =\exp [i \omega t] \frac{\left\langle\sum_{m, n} \exp \left[-i \mathbf{q} \cdot\left(\mathbf{r}_{n}(0)-\mathbf{r}_{m}(t)\right)\right]\right\rangle}{\left\langle\sum_{m, n} \exp \left[-i \mathbf{q} \cdot\left(\mathbf{r}_{n}(t)-\mathbf{r}_{m}(t)\right)\right]\right\rangle} \\
& =\exp [i \omega t] \frac{F(\mathbf{q}, t)}{S(\mathbf{q})} .
\end{aligned}
$$

The fraction in Eqn. 4.53 is called the Siegert relation [87]. $F(\mathbf{q}, t)$ is called the intermediate scattering function. The time Fourier transform of $F(\mathbf{q}, t)$ is

$$
\begin{aligned}
\frac{1}{2 \pi} \int F(\mathbf{q}, t) \exp [i \omega t] d t & =\frac{1}{2 \pi} \int\left\langle\sum_{m, n} \exp \left[-i\left(\mathbf{q} \cdot\left(\mathbf{r}_{n}(0)-\mathbf{r}_{m}(t)\right)-\omega t\right)\right]\right\rangle d t \\
& =\frac{1}{2 \pi} \iint\langle\rho(0,0) \rho(\mathbf{r}, t)\rangle \exp [-i(\mathbf{q} \cdot \mathbf{r}-\omega t)] d \mathbf{r} d t \\
& =S(\mathbf{q}, \omega) .
\end{aligned}
$$

If the dipoles are identical $\langle\rho(0,0) \rho(\mathbf{r}, t)\rangle$ is the space-time dipole-dipole density correlation function. $S(\mathbf{q}, \omega)$ is called the dynamic structure factor [86]. The static and dynamic structure factors relate spatiotemporal fluctuations in the field to spatiotemporal fluctuations in the density. Loosely, the static structure factor probes structure on length scales $q^{-1}$ whereas the dynamic structure factor reports on temporal fluctuations at length scales $q^{-1}$.

If the dipoles are identical, isotropic and the positions are uncorrelated, the cross terms in Eqn. $4.53(n \neq m)$ average to zero leaving

$$
\left|g_{1}(t)\right|=\left\langle\exp \left[-i \mathbf{q} \cdot\left(r_{n}(0)-r_{n}(t)\right)\right]\right\rangle .
$$

If a phase factor $\Delta \phi(t)$ is a Gaussian random variable with a zero mean then by definition

$$
\langle\exp [-i \Delta \phi(t)]\rangle=\exp \left[-\left\langle\Delta \phi^{2}(t)\right\rangle / 2\right] .
$$



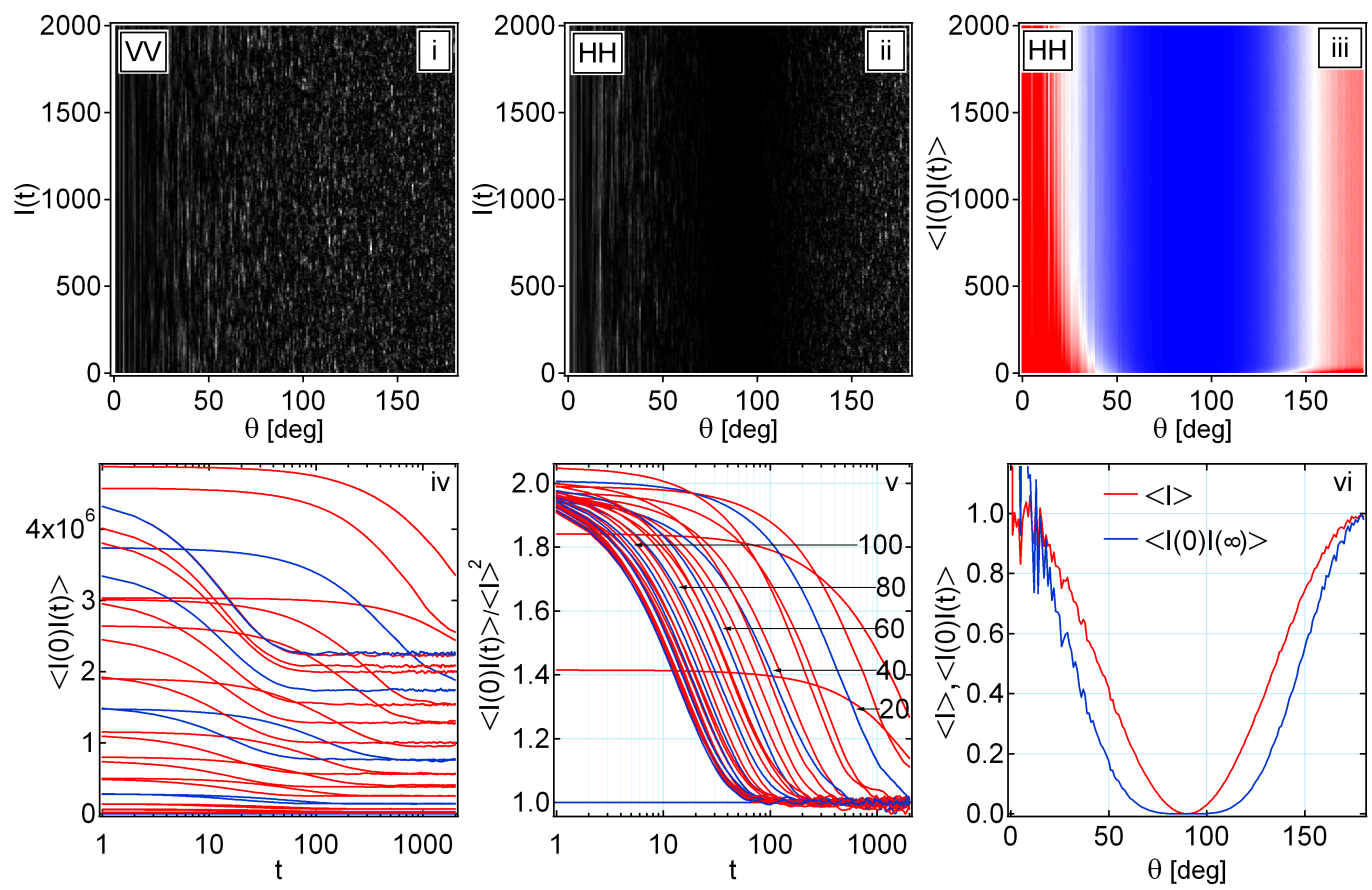

Figure 4.2: Simulated scattered intensity with time and angle in the i) VV and ii) $\mathrm{HH}$ optical configurations. iii-iv) Numerically calculated intensity correlations $\langle I(0) I(t)\rangle$ with angle for the simulated $\mathrm{HH}$ data. v) Numerically calculated normalised intensity correlations $\langle I(0) I(t)\rangle /\langle I\rangle^{2}$ with angle for the simulated HH data. vi) Angle dependence of $\langle I\rangle$ and $\langle I(0) I(\infty)\rangle$.

For free diffusion $\Delta \mathbf{r}_{n}(t)=\mathbf{r}_{n}(0)-\mathbf{r}_{n}(t)$ is a Gaussian random variable and

$$
\left|g_{1}(t)\right|=\left\langle\exp \left[-i \mathbf{q} \cdot \Delta r_{n}(t)\right]\right\rangle=\exp \left[-q^{2}\left\langle\Delta r^{2}(t)\right\rangle / 6\right]
$$

implying that for free diffusion [11]

$$
\frac{\langle I(0) I(t)\rangle}{\langle I\rangle^{2}}=\left[1+\exp \left[-2 q^{2} D t\right] .\right.
$$

The factor of $1 / 3$ comes from averaging $\mathbf{q} \cdot \Delta \mathbf{r}$ over a sphere. As indicated by Eqn. 4.60 dynamic light scattering can be used to determine the MSD of the diffusing dipoles (if they are identical, isotropic and their positions are uncorrelated).

The basic principles of static and dynamic light scattering are illustrated using simulation in Fig. 4.2. Here, light scattered from an ensemble of 100 dipoles with isotropic polarisability within a $1 \mathrm{~mm}^{3}$ scattering volume and undergoing free diffusion is simulated as a function of both time and scattering angle $\theta$. It was assumed that $\mathbf{k}_{i}=k \hat{\mathbf{k}}$ and that $\mathbf{k}_{f}=k(\sin \theta \hat{\mathbf{i}}+\cos \theta \hat{\mathbf{k}})$ - the plane containing $\mathbf{k}_{i}$ and $\mathbf{k}_{f}$ is called the scattering plane. 
The polariser and analyser were aligned so that either $\mathbf{n}_{i}=[0,1,0]^{T}$ and $\mathbf{n}_{f}=[0,1,0]^{T}$ or $\mathbf{n}_{i}=[1,0,0]^{T}$ and $\mathbf{n}_{f}=[\sin \theta, 0, \cos \theta]^{T}$. In the first configuration, both the polariser and analyser are perpendicular to the scattering plane. Because the scattering plane is often horizontal, this is referred to as the vertical-vertical or VV configuration. The second configuration is referred to as the horizontal-horizontal or $\mathrm{HH}$ configuration. Physical values of $k=1.3 \times 10^{7} \mathrm{~m}^{-1}, \alpha=1$ and $R=0.1 \mathrm{~m}$ were assumed. As seen in Fig. 4.2 i-ii in both the $\mathrm{VV}$ and $\mathrm{HH}$ configurations, the fluctuations in intensity increase with scattering angle. This is expected as a consequence of the $q$ dependence in the exponent of Eqn. 4.60. A decrease average intensity is observed around $\theta=90 \mathrm{deg}$ in $\mathrm{HH}$ average. This too is expected for dipole radiation - longitudinal electromagnetic waves are prohibited in free space. To quantify these observations a time dependent intensity correlation $\langle I(0) I(t)\rangle$ is calculated numerically using circular correlation and is illustrated in Fig. 4.2 iii for the $\mathrm{HH}$ data. This calculation is normally done in hardware experimentally. Fig. 4.2 iv shows vertical line profiles through Fig. 4.2 iii at $\theta=5 \mathrm{deg}$ increments. The correlation is observed to decay exponentially to a baseline value. The correlation is normalised with respect to the long time behaviour of the correlation $\langle I(0) I(\infty)\rangle=\langle I\rangle^{2}$. Here, it is seen that the resulting correlation has the form predicted by Eqn. 4.61. The average intensity $\langle I\rangle$ calculated from Fig. 4.2 ii and $\langle I(0) I(\infty)\rangle$ is compared in Fig. 4.2 vi. $\langle I\rangle$ and $\langle I(0) I(\infty)\rangle$ have been normalised to equal 1 at $\theta=0 \mathrm{deg}$. Not surprisingly $\langle I(0) I(\infty)\rangle \propto \cos ^{4} \theta$ and $\langle I\rangle \propto \cos ^{2} \theta$ as is expected for dipole radiation.

In the more general case, the scattered field will depend on the internal fields generated inside the scattering centres and refraction at the surface. In the Rayleigh-Gans approximation, valid when $|m-1|<<1$ and $2 \pi a|m-1|<<\lambda$ [88], where $a$ is a characteristic length of the scattering centre, these effects are neglected. In this approximation, the field scattered by isotropic fluctuations in refractive index $\underline{\underline{m}}^{2}(\mathbf{r})-\underline{\underline{I}}=\underline{\underline{\Delta}}(\mathbf{r}, t)$ is [11]

$$
E_{s}(t)=E_{0} \frac{\exp [i(k R-\omega t)]}{4 \pi R} k_{f}^{2} \int \exp [-i \mathbf{q} \cdot \mathbf{r}] \Delta \epsilon_{i, f}(\mathbf{r}, t) d \mathbf{r} .
$$

If the fluctuations are isotropic, the static structure factor and dynamic structure factor can be calculated from Eqn. 4.48 and 4.56 with $\rho(\mathbf{r}, t) \rightarrow \Delta \epsilon_{i f}(\mathbf{r}, t)$. The scattered intensity at small angles in the Rayleigh-Gans approximation is illustrated for three cases in Fig. 4.3 . Fig. 4.3 i shows the time averaged scattered intensity distribution for a solid sphere $r=0.5 \mu \mathrm{m}$, calculated numerically by summing up the contribution of scattering elements/dipoles arranged on a grid within a sphere and analytically (coloured contours, from [88]). Intensity is evaluated at $R=0.1 \mathrm{~m}$ and $k=1.3 \times 10^{7} \mathrm{~m}^{-1}$. The intensity is sharply peaked in the forward direction. Fig. 4.3 ii shows the averaged scattered intensity distribution for a random ensemble of solid ellipsoids $r_{x} \times r_{y} \times r_{z}=1 \times 0.5 \times 0.5 \mu \mathrm{m}$ with the long axis orientated along the $\hat{\mathbf{i}}$ direction. Not surprisingly, because of the Fourier relationship between the polarisability and scattered intensity, the scattered intensity becomes elongated along the $\hat{\mathbf{j}}$ axis. The butterfly pattern shown in Fig. 4.3 iii is sometimes observed in sheared polymer solutions and has been associated with 'critical concentration fluctuations' 12 . The inset in Fig. 4.3 iii shows the inverse Fourier transform of the scattered intensity which is proportional to $\langle\rho(0) \rho(r)\rangle$. The spatial variation in the dielectric 

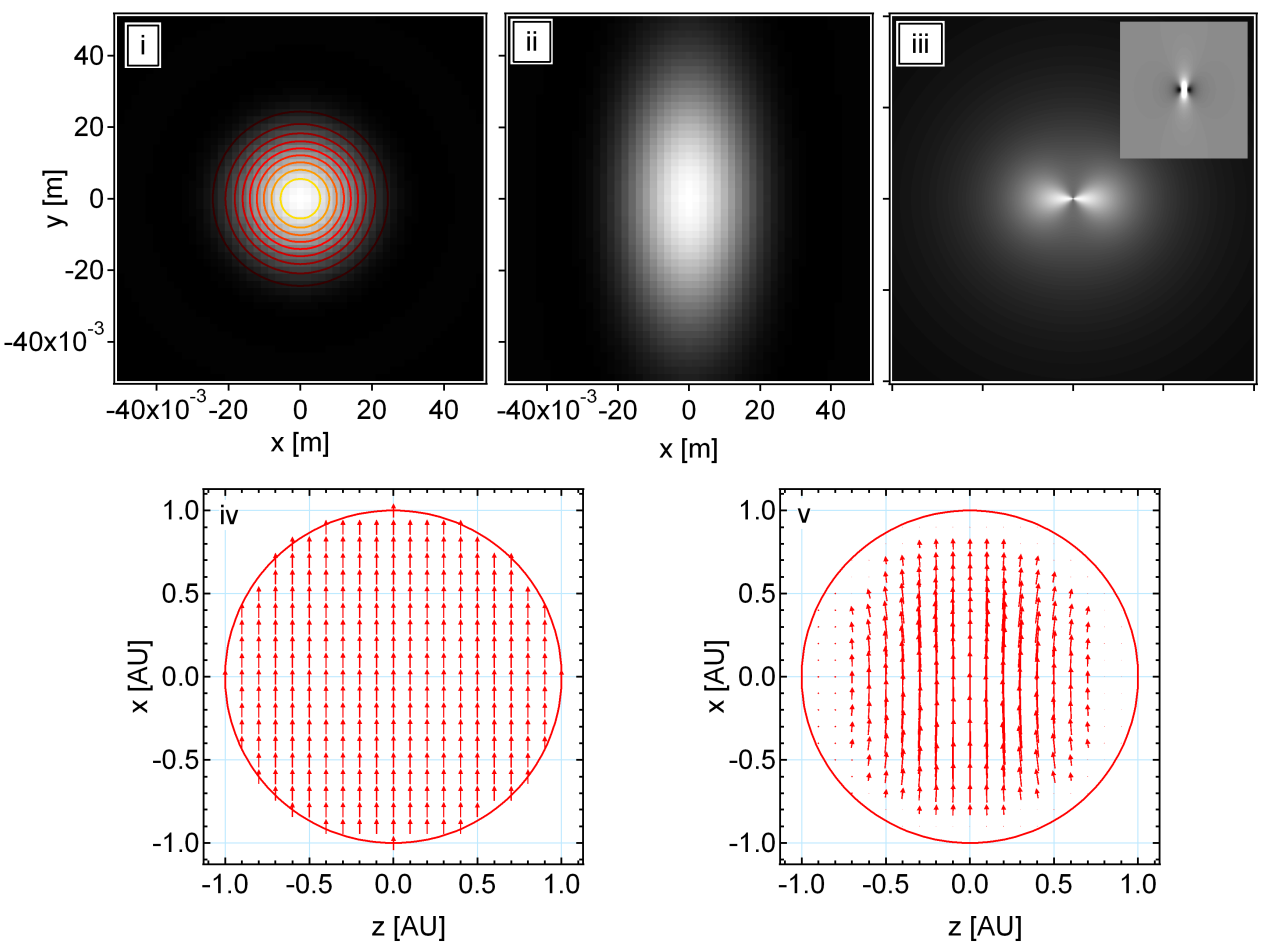

Figure 4.3: Small angle light scattered from a i) solid sphere, ii) solid ellipsoid and from iii) 'critical concentration fluctuations'. Near field dipole-dipole interaction in an infinite rod with iv) weak and v) strong interaction.

constant shows both positive and negative lobes suggesting regions of higher and lower polymer concentration. The study of the scattering patterns at small angles is called small angle light scattering (SALS).

The Rayleigh-Gans approximation is only valid when the interaction fields between scattering elements can be neglected. As noted above, this is only reasonable if $\mid m-$ $1 \mid<<1$ and $2 \pi a|m-1|<<\lambda$. Copic's model, which was developed to describe near field interactions in a polymer chain but is introduced here because it would appear (to the Author, at least) to be quite general, includes these near field interactions between dipoles/scattering elements extending the range of validity of the Rayleigh and RayleighGans models [12]. Here, the field between the dipole is mediated by an interaction tensor $\underline{\underline{T}}_{i j}$

$$
\underline{\underline{T}}_{i j}=\frac{1}{n} \frac{1}{r_{i j}^{3}}\left[3 \frac{\mathbf{r}_{i j} \mathbf{r}_{i j}}{r_{i j}^{3}}-\underline{\underline{I}}\right],
$$

where $\mathbf{r}_{i}$ is the location of the $i$ th dipole, $\mathbf{r}_{i j}=\mathbf{r}_{i}-\mathbf{r}_{j}$, and $n$ is the refractive index of the medium which controls the strength of the interaction. The dipole moment of the $i$ th 
dipole is given by

$$
\mathbf{p}_{i}=\underline{\underline{\alpha}}_{i} \cdot \mathbf{E}+\sum_{j} \underline{\underline{T}}_{i j} \cdot \mathbf{p}_{i}
$$

which is a generalisation of Eqn. 4.42 to include near field interactions. The system of linear equations described by 4.64 can be solved for $\mathbf{p}_{i}$. The dipole moment induced by light polarised in the $\hat{\mathbf{i}}$ direction in an infinite rod aligned in the $\hat{\mathbf{j}}$ direction with weak and strong interactions is shown in Fig. 4.3 iv and 4.3 v. In Fig. 4.3 iv dipole moments are aligned with the field and are spatially uniform as would be expected in the Rayleigh-Gans approximation. In Fig. $4.3 \mathrm{v}$ the dipole moment is strongest in the centre of the rod and is no longer directed along the incident field. The field, due to such a distribution of dipoles, could be calculated by summing the fields radiated by the scattering elements as was done above.

Mie solved Maxwell equations for isotropic spheres of arbitrary size and refractive index exactly. The solution is expressed in terms of the complex scattering amplitudes $S_{1}(\theta, m)$ and $S_{2}(\theta, m)$, where $\theta$ is the angle between the incident and the outgoing wave vectors. For a polarised incident field $\mathbf{E}=\tilde{A}_{x} \mathbf{E}_{x}+\tilde{A}_{y} \mathbf{E}_{y}$ the field scattered from an isolated sphere at a distant observation point $R \cos \theta \hat{\mathbf{k}}+R \sin \theta \hat{\mathbf{i}}$ is 88 ]

$$
\mathbf{E}_{s}(t)=E_{0} \frac{\exp [i k R]}{i k R}\left[\tilde{A}_{x} S_{2}(\theta, m) \mathbf{E}_{x}+\tilde{A}_{y} S_{1}(\theta, m) \mathbf{E}_{y}\right] .
$$

\subsubsection{Multiple scattering in the diffusion approximation}

In the previous section, it was assumed that light only interacted with the scattering centres once before detection. Dynamic light scattering in this regime is called photocorrelation spectroscopy (PCS) or quasi-elastic light scattering (it is also known as 'dynamic light scattering'). This assumption is only appropriate when the density of scattering centres is low. At higher densities, light may interact with scattering centres multiple times before detection. If multiple scattering is significant, the propagation of light can be treated as a diffusive process. In this diffusion approximation, the path of light through the highly multiple scattering medium can be represented by a random walk. Diffusing wave spectroscopy (DWS) is a dynamic light scattering technique which is based on this diffusion approximation.

Two length scales are important in highly multiple scattering media: the mean free path $l$ and the transport mean free path $l^{*} . l$ is the average distance between scattering events and is given by [87]

$$
l=\frac{1}{\rho \sigma_{s}} .
$$

$\rho$ is the number density of the scattering centres. $\sigma_{s}=\int R(\theta, \phi) \sin \theta d \theta d \phi$ is the total scattering cross-section. $R(\theta, \phi)$ is called the form factor. For isotropic spheres $R(\theta, \phi)=$ $\left|S_{1}(\theta)\right|^{2} \cos ^{2} \phi+\left|S_{2}(\theta)\right|^{2} \sin ^{2} \phi$ [88] (scattering amplitudes $S_{1}(\theta)$ and $S_{2}(\theta)$ are calculated 
according to Mie theory). $l^{*}$ is the average distance light travels before its direction is randomised. $l^{*}$ is related to $l$ by

$$
l^{*}=\frac{l}{\langle 1-\cos \theta\rangle},
$$

where the average in the denominator is weighted by the form factor of the scattering centres. The relation between $l$ and $l^{*}$ is illustrated using simulation in Fig. 4.4. Here, light propagating in the $\hat{\mathbf{k}}$ direction and polarised in the $\hat{\mathbf{j}}$ direction is scattering by a sphere at the origin in a direction $\sin \theta \cos \phi \hat{\mathbf{i}}+\sin \theta \sin \phi \hat{\mathbf{j}}+\cos \theta \hat{\mathbf{k}}$ with a probability determined by the form factor. The typical distribution of directions is shown in Fig. 4.4 i-iv for spheres of different radius $a$. Note that scattering is preferentially in the forward direction for the larger spheres. The weighted average $\langle 1-\cos \theta\rangle$ is calculated directly from the simulation. The ratio $l / l^{*}$ is shown in Fig. $4.4 \mathrm{v}$. As scattering anisotropy increases with $a$ the ratio $l^{*} / l$ increases - light must undergo more scattering events before its direction is randomised. $c l$ was calculated analytically and used to calculate $c l^{*}$ for fixed volume fraction scattering centre suspension. The constant of proportionality $c$ is related to the actual concentration of scattering centres present. This was not determined directly but can be inferred by comparing the Author's $l$ to the $l$ extracted data [87. With this scaling factor determined (once) $l^{*}$ can be determined. The simulated and analytic $l^{*}$ are in close agreement. On a length scale $l$ the direction of propagation is influenced by previous scattering events and the direction of propagation is not random. However, on a longer length scale $l^{*}$, which includes multiple scattering events, the direction of propagation is random and the path of light is that of a random walk.

Following Weitz and Pine [87, a typical light path through a highly scattering medium is illustrated in Fig. 4.5. The total path length $s$ between source and detector is

$$
s=\sum_{i=0}^{n}\left|\mathbf{r}_{i+1}+\mathbf{r}_{i}\right|=\frac{\mathbf{k}_{i}}{|\mathbf{k}|} \cdot\left[\mathbf{r}_{i+1}+\mathbf{r}_{i}\right]=N l .
$$

In DWS it is assumed that the time dependent multiple scattered field is of the form

$$
E(t)=\sum_{p} E_{p} \exp \left[i \phi_{p}(t)\right]
$$

$E_{p}$ represents the field amplitude associated with some path $p$ and time dependent phase shift $\phi_{p}(t)$ is introduced as the paths distort with time due to the motion of the scattering centres. The total field is a sum over all paths detected. By analogy to the single scattering case, the field correlation function when multiple scattering is present is given by

$$
\begin{aligned}
g_{1}(t)=\frac{\langle E(0) E(t)\rangle}{\left\langle|E|^{2}\right\rangle} & =\frac{1}{\langle I\rangle} \sum_{p} E_{p} \exp \left[i \phi_{p}(0)\right] \sum_{p} E_{p^{\prime}}^{*} \exp \left[-i \phi_{p^{\prime}}(t)\right] \\
& \approx \sum_{p} \frac{\left\langle I_{p}\right\rangle}{\langle I\rangle}\left\langle\exp \left[i \Delta \phi_{p}(t)\right]\right\rangle .
\end{aligned}
$$



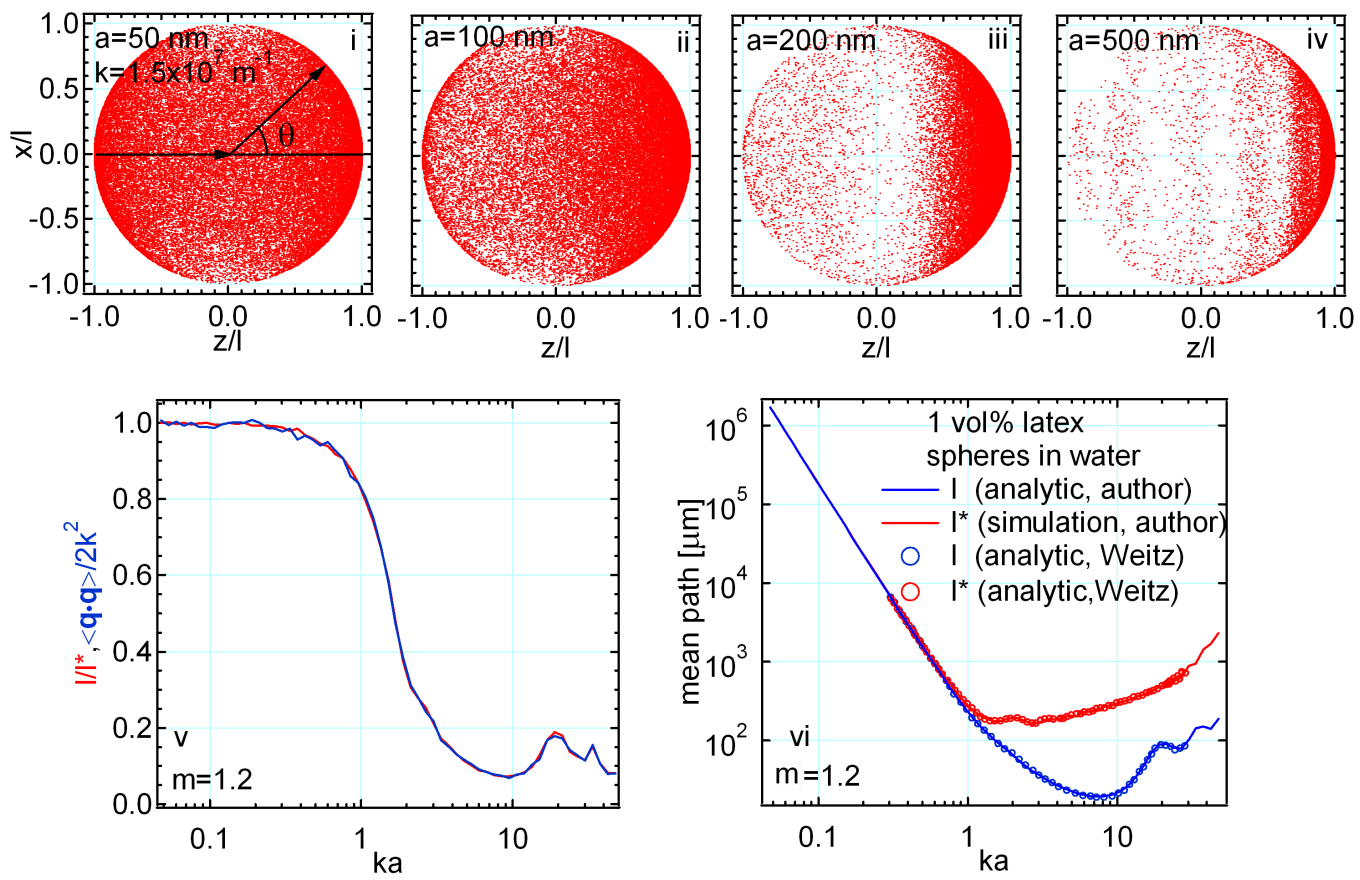

Figure 4.4: i-iv) Simulated distribution of scattering angles for scattering centres of radii $\mathrm{a}=50-500 \mathrm{~nm}$. v-vi) Comparison between simulated and analytically calculated $l$ and $l^{*}$ values for a $1 \%$ suspension of $m=1.2$ spheres. Analytic data were extracted from [87].

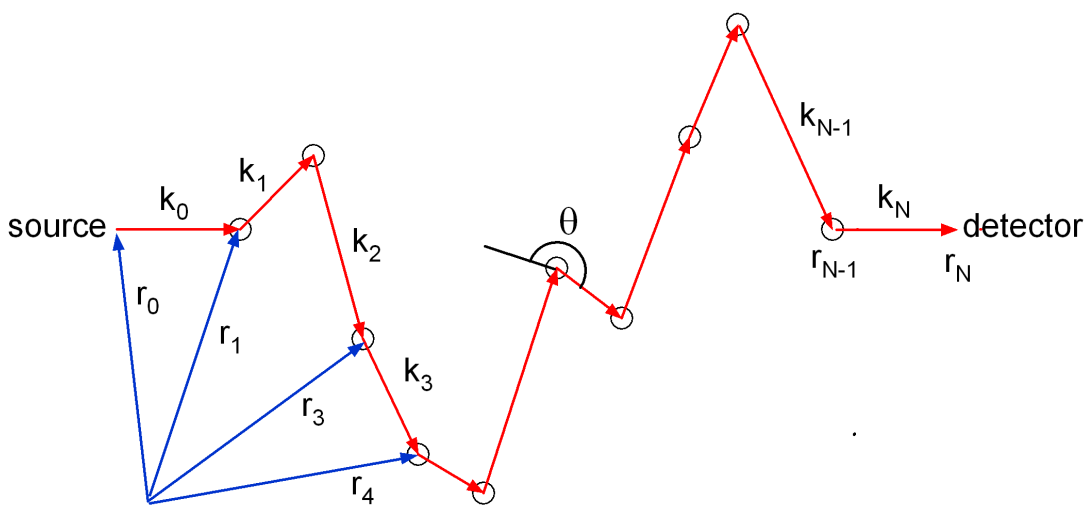

Figure 4.5: Multiply scattered light path through some turbid medium. The wave vectors $\mathbf{k}$ have the same magnitude and position vector $\mathbf{r}$ describe the instantaneous position of the scattering centres (adapted from [87]). 
It has been assumed that the fields from different light paths are uncorrelated. The absolute phase of the light scattering down the path like that indicated in Fig. 4.5 depends on the path length. If the length of the path changes the absolute phase will also change. $g_{1}(t)$ is expected to be sensitive to this change in phase. Using geometric arguments it can be shown that

$$
\begin{aligned}
\Delta \phi_{p}(t) & =\phi_{p}(t)-\phi_{p}(0)=\sum_{i=0}^{n} \mathbf{k}_{i}(t) \cdot\left[\mathbf{r}_{i+1}(t)-\mathbf{r}_{i}(t)\right]-\sum_{i=0}^{n} \mathbf{k}_{i}(0) \cdot\left[\mathbf{r}_{i+1}(0)-\mathbf{r}_{i}(0)\right] \\
& \approx \sum_{i=1}^{n} \mathbf{q}_{i}(t) \cdot \Delta \mathbf{r}_{i}(t) .
\end{aligned}
$$

If $\Delta \phi_{p}(t)$ is a Gaussian random variable with a zero mean then by definition $\left\langle\exp \left[i \Delta \phi_{p}(t)\right]\right\rangle=$ $\exp \left[\left\langle\Delta \phi_{p}^{2}(t)\right\rangle / 2\right]$ and the average phase shift is then

$$
\begin{aligned}
\left\langle\Delta \phi_{p}^{2}(t)\right\rangle & =\sum_{i=1}^{N} \mathbf{q}_{N}(t) \cdot \Delta \mathbf{r}_{i}(t) \sum_{j=1}^{N} \mathbf{q}_{j}(t) \cdot \Delta \mathbf{r}_{j}(t) \\
& \approx \frac{1}{3} N\left\langle q^{2}\right\rangle\left\langle\Delta r^{2}(t)\right\rangle .
\end{aligned}
$$

The quantity $\left\langle q^{2}\right\rangle=\langle\mathbf{q} \cdot \mathbf{q}\rangle$ depends on the form factor of the scattering centres. As illustrated in Fig. $4.4\left\langle q^{2}\right\rangle /\left(2 k^{2}\right)=l / l^{*}$. Recalling that $N=s / l$ [87]

$$
\left\langle\Delta \phi_{p}^{2}(t)\right\rangle=\frac{1}{3} \frac{s}{l} 2 k^{2} \frac{l}{l^{*}}=\frac{2}{3} n k^{2}\left\langle\Delta r^{2}(t)\right\rangle,
$$

where $n=s / l^{*} . n(<N)$ is the number of steps of stepsize $l^{*}$ in the path. Note that $l, l^{*}, N$ and $n$ are analogues of quantities $l, l_{K}, N$ and $N_{K}$ used to describe the conformation of a linear polymer. This equation relates the temporal phase statistics to the time dependent distortion of a random walk associated with the relative motion of the scattering centres.

The ratio $\left\langle I_{p}\right\rangle /\langle I\rangle$ can be viewed as a probability that light will scatter down a particular path from source to detection point. In the diffusion approximation, it is assumed that this path can be replaced by an equivalent random walk. The probability that light will diffuse from a source point to the detection point depends only on the number of steps $n$ in the path. This probability can be determined by simulation or analytically by solving a diffusion equation of the form [87]

$$
\frac{\partial G}{\partial n}-\frac{1}{3} l^{*} \nabla^{2} G=\delta\left(\mathbf{r}-\mathbf{r}_{0}\right) \delta n,
$$

with initial conditions $G=1$ at $n=0$ and $\mathbf{r}=\mathbf{r}_{0}$ and with absorbing boundary conditions. $G$ is a Green function solution to the diffusion equation. $G=G_{n}\left(\mathbf{r}_{i}, \mathbf{r}_{o}, l^{*}\right)$ describes the probability that light will diffuse from a source point $\mathbf{r}_{i}$ to a detection point $\mathbf{r}_{o}$ in $n$ steps. Note that the source point for diffusion is usually taken to be $\left|\mathbf{r}_{i}\right|=l^{*}$ inside the scattering medium reflecting the fact that light must propagate a distance $l^{*}$ before diffusion begins to occur and the detection point is taken at a distance $\left|\mathbf{r}_{o}\right|=\gamma_{0} l^{*}$ inside the scattering 
medium, where $\gamma_{0}$ is a factor of order unity that is related to diffusion of light near an interface. Simulation based on one-dimensional particle diffusion is used to build up a $G_{n}\left(x_{i}, x_{o}, l^{*}\right)$ in Fig. 4.5 and illustrates the essential physics. A particle is introduced at $x_{i}=0.1 n=0$ into a one-dimensional slab of width $L=1$. The particle diffuses a random distance drawn from a Gaussian distribution of width $l^{*}=0.1$ and mean zero in each $n$-step adding 1 to each point on the two-dimensional $x \times n$ grid where the particle settles. Eventually the particle is absorbed at the boundaries. This is repeated and the grids are co-added $100,10^{4}$ and $10^{6}$ times in Fig. 4.5 ii-iv and converge to the analytic path distribution in Fig. $4.5 \mathrm{v}$, vii-viii. The probability $G_{n}\left(x_{o}, x_{i}, l^{*}\right)$ that the particle will diffuse from $x_{i}$ to $x_{o}$ in $n$ steps is given by a vertical line at $x=x_{o}$ through the path distribution. The analytic solution for the one-dimensional slab was calculated according to 89 ]

$$
G_{n}\left(x_{i}, x_{o}, l^{*}\right)=\frac{2}{L} \sum_{m=1}^{\infty} \sin \left[\frac{m \pi x_{i}}{L}\right] \sin \left[\frac{m \pi x_{o}}{L}\right] \exp \left[-m^{2} \frac{n}{n_{0}}\right],
$$

where $n_{0}=3 L^{2} / \pi^{2} l^{* 2}$ which is a solution to Eqn. 4.77. In this diffusion approximation, the field correlation function can be rewritten

$$
g_{1}(t)=\sum_{n=1}^{\infty} G_{n}\left(\mathbf{r}_{i}, \mathbf{r}_{o}, l^{*}\right) \exp \left[\frac{1}{3} k^{2} n\left\langle\Delta r^{2}(t)\right\rangle\right] .
$$

Two geometries are commonly used in DWS: 1) backscattering and 2) transmission. In the backscattering geometry, light is collected from the incident face of the slab and it is assumed that $x_{o}=\gamma_{0} l^{*}$. In the transmission geometry, light is collected from the opposing face of the slab - here it is assumed that $x_{o}=L-\gamma_{0} l^{*}$ [90] [89]. In the backscattering geometry, the correlation is dominated by short paths that preferentially 'samples' the diffuse medium near the incident face. In the transmission geometry, light must diffuse entirely through the slab before it is detected - more uniformly sampling the medium.

\subsubsection{Matrix formulations}

The Jones and Mueller/Stoke calculi can be used to represent changes of polarisation that can occur during scattering. The general form of the scattering matrix for an isolated scattering centre is [88] 12

$$
\mathrm{T}_{J}=\frac{\exp [i k R]}{i k R}\left[\begin{array}{ll}
S_{2}(\theta, \phi) & S_{3}(\theta, \phi) \\
S_{4}(\theta, \phi) & S_{1}(\theta, \phi)
\end{array}\right] .
$$

This scattering matrix can be calculated from the spatial polarisability/dielectric constant variation. If the particle is rotationally symmetric, the off axis components $S_{3}(\theta, \phi)=$ $S_{4}(\theta, \phi)=0$. If the scattering centre is not rotationally symmetric, the off axis components are non zero [88]. Because of this orientation dependence, these terms may differ from centre to centre in an ensemble. There is no Jones matrix representation for such an 


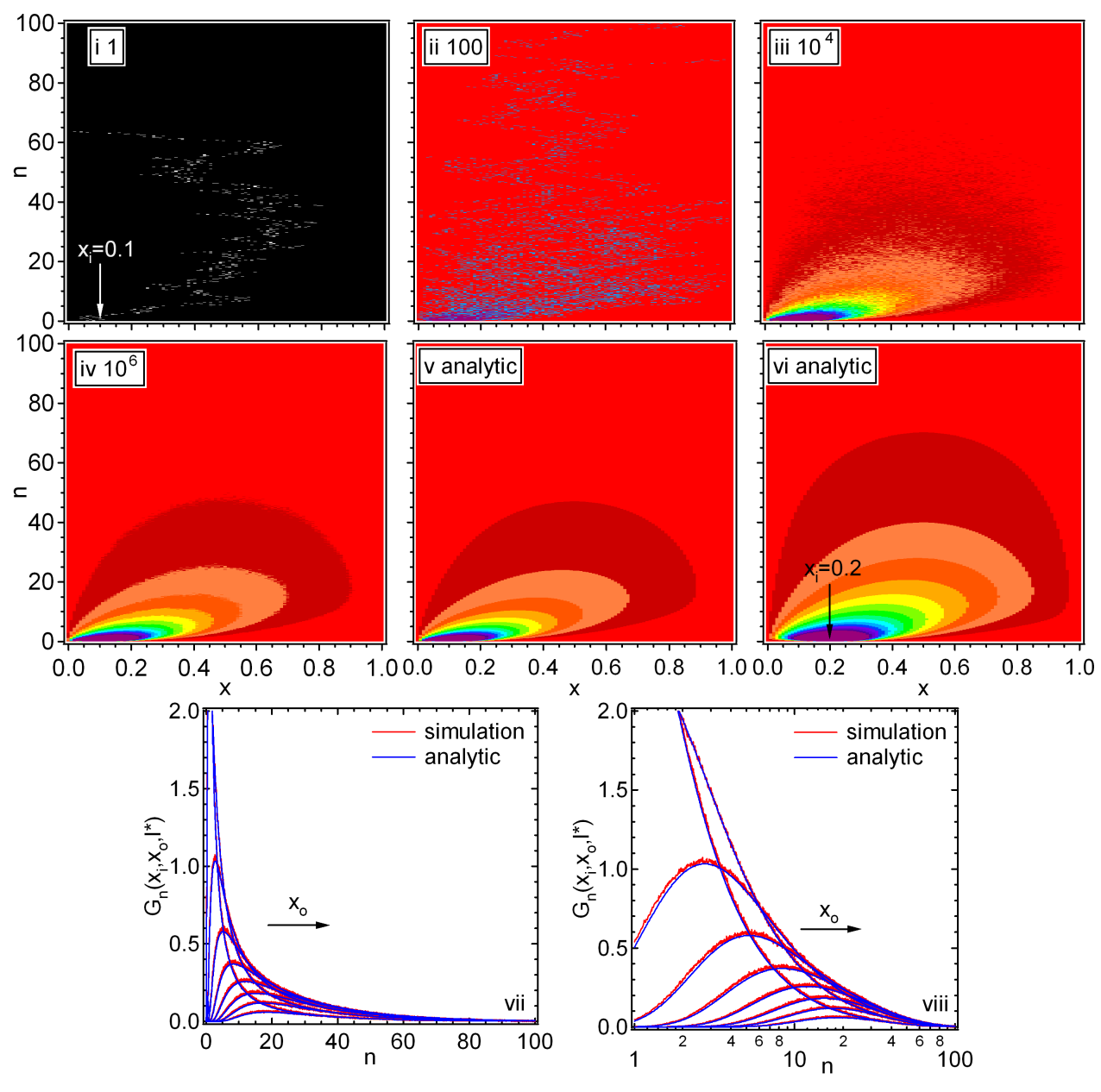

Figure 4.6: i-iv) Simulation showing that the diffusion of test particles on a grid, with appropriate boundary conditions, converges to the propagator $G_{n}\left(\mathbf{r}_{i}, \mathbf{r}_{o}, l^{*}\right)$ calculated analytically in v). vi) Analytic propagator with a different choice of parameters for comparison. vii-viii) Direct comparison between the simulated and analytic propagator calculated according to Eqn. 4.78 . 


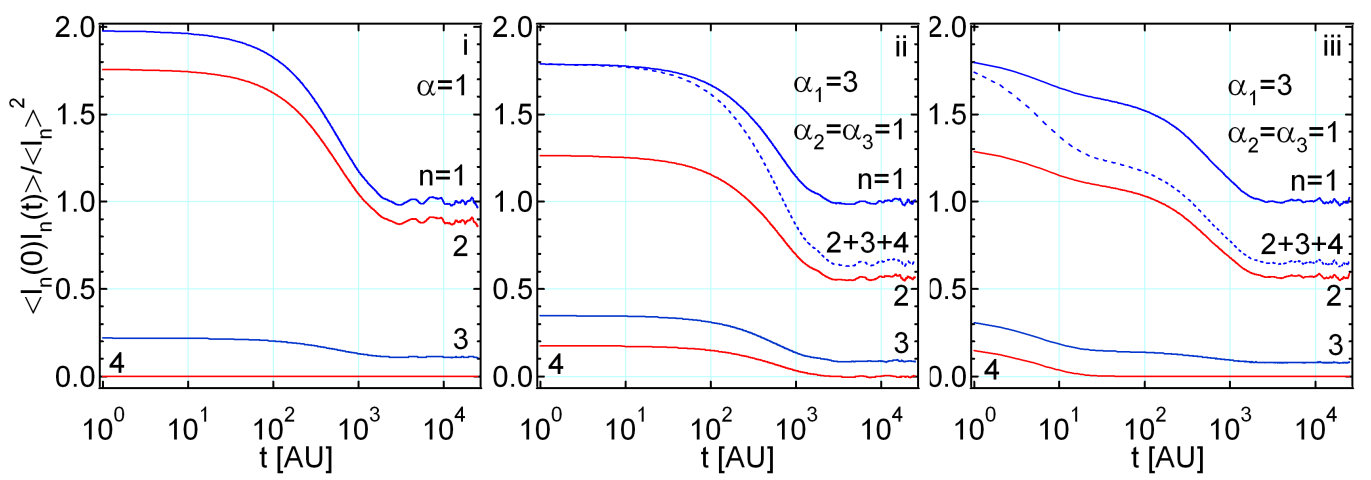

Figure 4.7: Simulated intensity correlation for each of the four components of the Stokes vector for i) isotropic Rayleigh scattering centres undergoing diffusion, ii) anisotropic Rayleigh scattering centres undergoing slow translational diffusion and iii) anisotropic Rayleigh scattering centres undergoing slow translational diffusion and fast rotational diffusion.

ensemble. The Mueller calculus must be used. The effects of orientation are examined in Fig. 4.7 using simulation. Here, the field from 100 scattering centres in a $1 \mathrm{~mm}{ }^{3}$ scattering volume is summed at a distant observation point and the correlation $\left\langle I_{n}(0) I_{n}(t)\right\rangle$ is calculated for each element $(n)$ of the Stokes vector for the scattering centres undergoing translational (and in the Fig. 4.7 iii rotational) diffusion in the Rayleigh approximation. The scattering matrix was calculated from the polarisability using methods outlined in [88. It was assumed that incident light was propagating in $\hat{\mathbf{k}}$ direction with a polarisation $\mathbf{E}=E_{0} \hat{\mathbf{i}}+E_{0} \hat{\mathbf{j}}$ and was detected in the $\hat{\mathbf{i}}-\hat{\mathbf{k}}$ plane at a scattering angle of $\theta=\pi / 4$. The correlations show time dependence - this is not surprising as this is the time dependence that is measured in PCS. The long time behaviour of the correlation is $\left\langle I_{n}\right\rangle^{2}$. In Fig. 4.7 i the scattering centres are isotropic and $\left\langle I_{1}\right\rangle^{2}=\left\langle I_{2}\right\rangle^{2}+\left\langle I_{3}\right\rangle^{2}+\left\langle I_{4}\right\rangle^{2}$ - the scattered light is completely polarised. In Fig. 4.7 ii the scattering centres are isotropic and randomly orientated. Here $\left\langle I_{1}\right\rangle^{2}>\left\langle I_{2}\right\rangle^{2}+\left\langle I_{3}\right\rangle^{2}+\left\langle I_{4}\right\rangle^{2}$. The scattered light is partially polarised randomly orientated anisotropic scattering centres depolarise the initially polarised light. Finally, in Fig. 4.7 iii the scattering centres are allowed to undergo 'fast' rotation diffusion in addition to the 'slow' translational diffusion. This modifies the time dependence of the correlation.

\subsubsection{Scattering birefringence and dichroism}

For spherical isotropic particles at a scattering angle $\theta=0, S_{1}(0)=S_{2}(0)=S(0)$. Expanding the Mie theory $S(0)$ in terms of dimensionless parameter $k a$ gives 88

$$
S(0)=i \frac{2}{3}(k a)^{3} \bar{\alpha}-\frac{2}{3}(k a)^{6} \bar{\alpha}^{2}+i O(k a)^{5}+O(k a)^{7} .
$$


The real part of the resulting scattering matrix produces an absolute phase shift in the incident light while the imaginary part, which is only significant when $k a$ is large, produces attenuation. Because a differential phase shift or differential attenuation is required for birefringence or dichroism, these spherical isotropic particles are neither. Fuller [12] notes that Eqn. 4.81 can be reexpressed

$$
S_{i j}(0)=i \frac{2}{3}(k a)^{3} \alpha_{i j}-\frac{2}{3}(k a)^{6} \alpha_{i k} \alpha_{i j}
$$

Consequently, anisotropies in the polarisability tensor of the scattering centres can lead to birefringence and dichroism.

\subsection{Selected rheo-optical properties of polymer like systems}

Following Fuller [12] in the model of Kuhn-Grun, each of the Kuhn monomers in the polymer strand are assumed to be rod-like non-interacting (in the near field sense) dipoles with uniaxial polarisability

$$
\underline{\underline{\alpha}}=\alpha_{2} \underline{\underline{I}}+\left(\alpha_{1}-\alpha_{2}\right) \mathbf{u u},
$$

where $\mathbf{u}$ is a director which describes the orientation of the dipole. The average polarisability of a macromolecule of $N_{K}$ Kuhn segments of length $l_{K}$ is

$$
\underline{\underline{\bar{\alpha}}}=N_{K} \alpha_{2} \underline{\underline{I}}+N_{K}\left(\alpha_{1}-\alpha_{2}\right)\langle\mathbf{u u}\rangle \text {. }
$$

Using the Lorentz-Lorenz equation (Eqn. 4.9) the refractive index of an ensemble of these macromolecules is

$$
\underline{\underline{n}}=\frac{4 \pi \nu}{18} \frac{\left(\bar{n}^{2}+2\right)^{2}}{\bar{n}}\left[\alpha_{2} \underline{\underline{I}}+\left(\alpha_{1}-\alpha_{2}\right)\langle\mathbf{u u}\rangle\right],
$$

where $\nu$ is the number density of monomers and $\bar{n}$ is the average refractive index . At equilibrium there is no preferred direction for the director $\mathbf{u}$ and $\langle\mathbf{u u}\rangle=\underline{\underline{I}}$. Under deformation or flow conditions this is no longer the case. The Kuhn-Grun distribution function $P(\mathbf{u}, \mathbf{R})$ gives the probability of an orientation $\mathbf{u}$ for an end-to-end vector $\mathbf{R}$. This relation links the tensor $\langle\mathbf{u u}\rangle$ to the tensor $\langle\mathbf{R R}\rangle$ and is of the form $\langle\mathbf{u u}\rangle=A+B\left\langle R^{2}\right\rangle+C\langle\mathbf{R R}\rangle$ and leads to a refractive index

$$
\underline{\underline{n}}=\frac{2 \pi \nu}{15} \frac{\left(n^{2}+2\right)^{2}}{\bar{n}}\left[\alpha_{1}-\alpha_{2}\right] \frac{1}{N_{K} l_{K}^{2}}\langle\mathbf{R R}\rangle+\text { isotropic terms. }
$$

According to the Rubbery Network model $\underline{\underline{\sigma}}=3 \nu k_{B} T /\left(N_{K} l_{K}^{2}\right)\langle\mathbf{R R}\rangle$. This can be combined with Eqn. 4.86 to give

$$
\begin{aligned}
\underline{\underline{n}} & =\frac{2 \pi}{45 k_{B} T} \frac{\left(\bar{n}^{2}+2\right)^{2}}{\bar{n}}\left[\alpha_{1}+\alpha_{2}\right] \times \underline{\underline{\sigma}}+\text { isotropic terms } \\
& =C \underline{\underline{\sigma}}+\text { isotropic terms. }
\end{aligned}
$$


Eqn. 4.88 relates the refractive index to stress. This relation is called the stress-optic rule and has been demonstrated to show wide validity for polymer melts and concentrated polymer solutions. The constant of proportionality is called the stress-optic coefficient and is typically of the order of $C \approx 10^{-9}-10^{-7} \mathrm{~Pa}^{-1}$.

The stress-optic rule implies that the refractive index tensor $\underline{\underline{n}}$, for simple shear flow $\mathbf{v}=v_{0} y \hat{\mathbf{i}}$, is of the form Eqn. 4.89 (LHS). Flow induces a biaxial anisotropy in the refractive index tensor. The refractive index tensor $\underline{\underline{n}}$ (and $\underline{\underline{\sigma}},\langle\mathbf{R R}\rangle$ and $\langle\mathbf{u u}\rangle$ ) can be diagonalised by the rotation transformation

$$
\left[\begin{array}{ccc}
n_{11} & n_{21} & 0 \\
n_{21} & n_{22} & 0 \\
0 & 0 & n_{33}
\end{array}\right]=\left[\begin{array}{ccc}
\cos \chi & \sin \chi & 0 \\
-\sin \chi & \cos \chi & 0 \\
0 & 0 & 1
\end{array}\right]\left[\begin{array}{ccc}
n_{11}^{\prime} & 0 & 0 \\
0 & n_{22}^{\prime} & 0 \\
0 & 0 & n_{33}^{\prime}
\end{array}\right]\left[\begin{array}{ccc}
\cos \chi & -\sin \chi & 0 \\
\sin \chi & \cos \chi & 0 \\
0 & 0 & 1
\end{array}\right] .
$$

That is, the primed 'principle' reference frame in which the refractive index tensor is diagonal, is rotated with respect to the unprimed lab frame by an angle $\chi$ about the vorticity axis $\nabla \times \mathbf{v}$ in simple shear flow conditions. This implies that the shear stress $\sigma$ and first $N_{1}$ and second $N_{2}$ normal force differences can be expressed:

$$
\begin{aligned}
\sigma & =\frac{1}{2 C}\left(n_{22}^{\prime}-n_{11}^{\prime}\right) \sin 2 \chi, \\
N_{1} & =\frac{1}{C}\left(n_{22}^{\prime}-n_{11}^{\prime}\right) \cos 2 \chi, \\
N_{2} & =\frac{1}{C}\left[\left(n_{33}^{\prime}-n_{11}^{\prime}\right)-\left(n_{22}^{\prime}-n_{11}^{\prime}\right) \cos ^{2} \chi\right] .
\end{aligned}
$$

As noted earlier, an optical anisotropy can be measured using ellipsometry - these optical measurements can be used to infer the mechanical stress if the stress-optic law is valid and the stress-optic coefficient is known. The effect of this optical anisotropy on the polarisation state depends on the direction of propagation through the medium. The vorticity axis is always a principle direction of the refractive index tensor in simple shear flow conditions. The polarisation state of light directed down this axis is only influenced by $n_{11}^{\prime}$ and $n_{22}^{\prime}$. The Jones matrix describing the effect of this anisotropy on the polarisation state of light can be determined by applying a rotation transformation to matrix

$$
T_{J}=\left[\begin{array}{cc}
\cos \chi & \sin \chi \\
-\sin \chi & \cos \chi
\end{array}\right]\left[\begin{array}{cc}
1 & 0 \\
0 & \exp [i \delta]
\end{array}\right]\left[\begin{array}{cc}
\cos \chi & -\sin \chi \\
\sin \chi & \cos \chi
\end{array}\right]
$$

where

$$
\delta=\delta_{y^{\prime}}-\delta_{x^{\prime}}=\frac{2 \pi\left(n_{22}^{\prime}-n_{11}^{\prime}\right) d}{\lambda}=\frac{2 \pi \Delta n d}{\lambda} .
$$

The parameters $\delta$ and $\chi$ can be measured using ellipsometry. If the stress optic rule is valid and the stress optic coefficient $C$ is known, an optical measurement of $\chi$ and $\delta$ can be used to infer $\sigma$ and $N_{1}$. In order to measure $N_{2}$ optically, an additional measurement 
of $\left(n_{33}^{\prime}-n_{11}^{\prime}\right)$ is required. Optical determination of $\left(n_{33}^{\prime}-n_{11}^{\prime}\right)$ is less straightforward as a component of the direction of propagation must be collinear with the $n_{11}^{\prime}$ axis which is not fixed with respect to the lab frame.

Again following Fuller [12], according to the Kuhn-Grun model birefringence $\Delta n=$ $n_{22}^{\prime}-n_{11}^{\prime}$ is

$$
\Delta n=\frac{3}{5}\left[\alpha_{1}-\alpha_{2}\right] \frac{R^{2}}{N_{K} l_{K}^{2}}
$$

Copic's model, which includes near field interactions between the monomers which are excluded in the Kuhn-Grun model, adds a correction that depends on the difference between the refractive index of the polymer and solvent $n_{s}$

$$
\Delta n=\underbrace{\frac{3}{5}\left[\alpha_{1}-\alpha_{2}\right]\left[\frac{R}{\sqrt{N_{K}} l_{K}}\right]^{2}}_{\text {intrinsic }}+\underbrace{\left[\frac{9 M}{4 \pi \rho N_{A}}\right] \frac{1}{\left(\sqrt{N_{K}} l_{K}\right)^{3}}\left[n_{s} \frac{\bar{n}-n_{s}}{\bar{n}^{2}-2 n_{s}^{2}}\right]\left[\frac{R}{\sqrt{N_{K}} l_{K}}\right]}_{\text {form }}+\ldots
$$

where $M$ and $\rho$ are the polymer molecular weight and density and $N_{A}$ is Avogadro's number. The intrinsic term is that given by the Kuhn-Grun model and may be positive or negative depending on the sign of the stress-optic constant. The form contribution to $\Delta n$ is always positive. The presence of form birefringence can complicate the interpretation of the measured $\delta$.

The static structure factor can be calculated by considering the chain to be a collection of connected dipoles of isotropic polarisability. In general, the structure factor has the form 91 ]

$$
S(q) \simeq \frac{S(0)}{1+q^{2} \xi^{2}}, \quad \mathrm{q} \xi<1,
$$

where $\xi$ is a characteristic correlation length. In dilute solutions $\xi=R_{G} / \sqrt{3}$ where $R_{G}$ is the radius of gyration of the polymer strand. For an ideal polymer $R_{G}^{2}=N l / 6$ [ $]$. Measurements of $S(\mathbf{q})$ can be used to determine the size of the polymer. In semi-dilute solutions the polymer chains overlap. Excluded volume interactions prevent complete interpenetration. In this case $\xi$ is a measurement of the degree of overlap. The structure factor for dilute polymer solutions for all $q$ is 91 ]

$$
S(q)=\frac{1}{q^{4} R_{G}^{4}}\left[\exp \left[-q^{2} R_{g}\right]-1+q^{2} R_{g}\right]
$$

and is shown for various $N\left(\propto \sqrt{R_{G}}\right)$ in Fig. 4.8. This result is simulated using two methods. In the first case, the field produced at a distant detection point by an ensemble of dipoles connected by a random walk is summed to calculate an intensity (red curves). In the second case, the dipole-dipole correlation function $\langle\rho(0) \rho(\mathbf{r})\rangle$ is simulated for an ensemble of random walks and the Fourier transform calculated from the simulated $\langle\rho(0) \rho(\mathbf{r})\rangle$ 

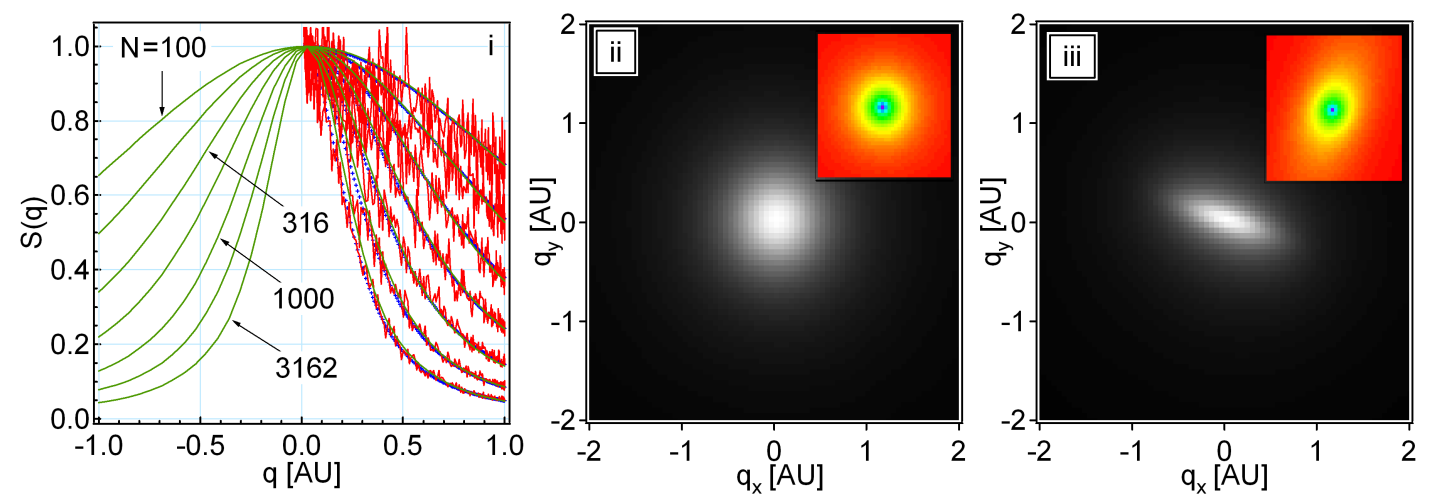

Figure 4.8: i) Simulated structure factor for an ensemble of isotropic dipoles, connected by a random walk, of length $N$ was calculated by summing the radiated field (red) or by considering the Fourier transform of the dipole-dipole correlation function (blue) and is compared to the analytic structure factor given by Eqn. 4.98. Two-dimensional representation of the scattered intensity (structure factor) a dilute polymer solution was calculated from the Fourier transform of the dipole-dipole density correlation function (shown inset) for ii) isotropic and iii) anisotropic distribution of dipoles in a random walk.

(green curves). A two-dimensional representation of $S(\mathbf{q})$ calculated from $\langle\rho(0) \rho(\mathbf{r})\rangle$ for chains of length $N_{K}=1000$ is shown in Fig. 4.8 ii and Fig. 4.8 iii. In Fig. 4.8 iii the chains have been sheared slightly in the $q_{y}$ direction. This introduces an anisotropy into the $\langle\rho(0) \rho(\mathbf{r})\rangle$ and $S(\mathbf{q})$. This anisotropy can be measured using SALS.

In dilute solutions, the polymer strands undergo thermal diffusion with an effective diffusion constant $D=k_{B} T /\left(6 \pi \eta_{s} R_{h}\right)$ where $R_{h}$ is the hydrodynamic radius of the polymer and $\eta_{s}$ is the solvent viscosity [91. This diffusion constant can be measured using DLS using the relation $\left|g_{1}(t)\right|=\exp \left[-q^{2} D t\right]$ and $R_{h}$ estimated from $D$. In semi-dilute solutions, the chains are constrained to diffuse within a tube produced by the surrounding chains. The confined snake model introduced in Chapter 1 suggests that the motion of the confined chain might loosely be characterised by two diffusion constants associated with the fast 'thrashing' mode and slow reptation modes. This would lead to a field correlation of the form

$$
\left|g_{1}(t)\right|=\underbrace{A \exp \left[-t / \tau_{f}\right]}_{\text {fast mode }}+\underbrace{B \exp \left[-t / \tau_{s}\right]}_{\text {slow mode }} .
$$

This model is too simplistic in that it treats the motion of the monomer dipole as being independent and excludes viscoelastic effects. To calculate $g_{1}(t)$ the dipole-dipole timespace correlation must be calculated 92 ]

$$
\left|g_{1}(t)\right|=\frac{\langle\rho(0,0) \rho(\mathbf{r}, t)\rangle}{\left\langle|\rho(\mathbf{r}, t)|^{2}\right\rangle}=\underbrace{A \exp \left[q^{2} D_{c} t\right]}_{\text {fast mode }}+\underbrace{\sum B_{i} \exp \left[-t / \tau_{i}\right]}_{\text {slow mode }} .
$$




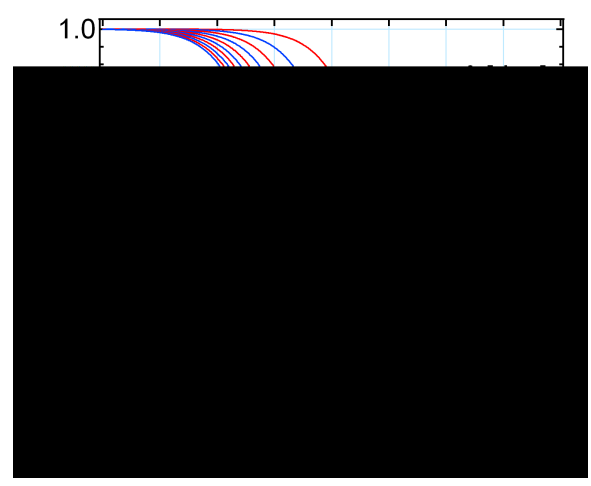

Figure 4.9: Angle dependence of $\left|g_{1}(t)\right|^{2}$ for an entangled polymer solution characterised by a single relaxation mode.

The slow mode depends on the longitudinal relaxation modulus $M(t)=\sum_{i} M_{i} \exp \left[-t / \tau_{i}\right]$ of the solution. In shear rheology, the relation between shear stress and shear strain (or strain rate) is studied. In extensional rheology, the relationship between stress and extensional strain is studied (extensional rheometry is difficult to implement experimentally). In the linear regime, a shear stress relaxation modulus $G(t)$ or extensional stress relaxation modulus $K(t)$ can be measured. The longitudinal modulus is related to both the shear and extensional modulus by $M(t)=K(t)+4 / 3 G(t)$ [92]. The fast mode depends on $D_{c}$ which is called the cooperative diffusion constant. The prefactors $A=a+b t$ and $B_{i}$ (time independent) are functions of $q, D_{c} M_{i}, \tau_{i}$, osmotic pressure and intermolecular potential. If $M(t)$ is characterised by a single relaxation mode, the ratio of the prefactor $A / B$ is a constant. Like the correlation predicted by the confined snake model, the correlation described by Eqn. 4.100 is bimodal. The characteristic time of the fast mode shows $q$ dependence whereas the characteristic time of the slow mode does not. Correlations predicted according to this model with scattering angle, for $A=B=0.5$ is shown in Fig. 4.9. The effects of shear flow on these characteristic times are investigated in Chapter 8. 


\section{Chapter 5}

\section{Examining the flow behaviour of wormlike micelles using rheometry and visualisation}

\subsection{Introduction}

In this first experimental chapter, three wormlike micelles solution systems are introduced and their rheology is examined using both conventional mechanical rheometry and direct visualisation. These observations are compared to predictions and observations discussed in Chapter 3. Some of these solutions will be re-examined in later chapters using other methods. In this chapter, the linear and nonlinear rheometry of the three systems is contrasted and compared to the predictions of the reptation-reaction model. The transient nonlinear rheometry of selected samples are examined for evidence of stress fluctuations/rheo-chaos and a possible dependence on surfactant supplier and flow geometry is considered. This rheometry work is followed with direct observations of the equilibrium low temperature behaviour and shear induced turbidity in three experimental flow geometries.

\subsection{Experimental}

The rheology of three wormlike micelles systems is examined in this chapter in detail. These systems were selected for their stability at room temperature $\left(T=22{ }^{\circ} \mathrm{C}\right)$. All three are composed of the cationic surfactant cetylpyridinium chloride $(\mathrm{CPCl})$ and counterion sodium salicylate ( $\mathrm{NaSal})$ in water with or without the salt sodium chloride $(\mathrm{NaCl})$. The $\mathrm{CPCl}$ and $\mathrm{NaSal}$ in water system has been studied extensively by Rehage and Hoffman [93. The primary system used in this thesis, RHB, is composed of $[\mathrm{CPCl}] /[\mathrm{NaSal}]=2$ at variable weight fraction $\phi=\phi_{\mathrm{CPCl}+\mathrm{NaSal}}$ in a stock $[\mathrm{NaCl}]=0.5 \mathrm{M}$ brine solution. This 


\begin{tabular}{|c|c|c|c|c|}
\hline system & conditions & variable(s) & chapter & references \\
\hline RHB & $\begin{array}{l}{[\mathrm{CPCl}] /[\mathrm{NaSal}]=2} \\
\text { in aqueous }[\mathrm{NaCl}]=0.5 \mathrm{M}\end{array}$ & $\begin{array}{l}\phi_{\mathrm{CPCl}+\mathrm{NaSal}} \\
{[\mathrm{wt} . \%]}\end{array}$ & $5,6,7,8$ & 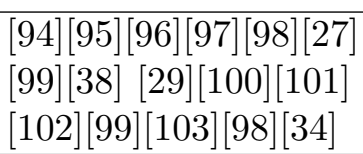 \\
\hline RHA & $\begin{array}{l}{[\mathrm{CPCl}]=0.1 \mathrm{M}+[\mathrm{NaSal}]} \\
\text { in water }\end{array}$ & $\begin{array}{l}{[\mathrm{NaSal}]} \\
\text { temperature }\end{array}$ & 5,6 & $\begin{array}{l}[28][104][26] 105][106] \\
[93][13] 33] 32] 107]\end{array}$ \\
\hline $\mathrm{RHC}$ & $\begin{array}{l}{[\mathrm{CPCl}]=0.1 \mathrm{M}+[\mathrm{NaSal}]=0.05 \mathrm{M}} \\
\text { in aqueous }[\mathrm{NaCl}]\end{array}$ & {$[\mathrm{NaCl}]$} & 5,6 & \\
\hline
\end{tabular}

Table 5.1: Composition of the three wormlike micelles systems studied. The cetylpyridinium chloride monohydrate used was provided by Sigma-Aldrich.

system is well studied. Often this system is described in terms of the surfactant weight fraction $\phi_{\mathrm{CPCl}}$. This weight fraction is related to the 'dry solid' weight fraction $\phi_{\mathrm{CPCl}+\mathrm{NaSal}}$ used here by $\phi_{\mathrm{CPCl}}=0.78 \times \phi_{\mathrm{CPCl}+\mathrm{NaSal}}$. The second system examined, RHA, is composed of $[\mathrm{CPCl}]=0.1 \mathrm{M}+[\mathrm{NaSal}]=0.030-0.631 \mathrm{M}$ in water. The final system, $\mathrm{RHC}$, is composed of $[\mathrm{CPCl}]=0.1 \mathrm{M}+[\mathrm{NaSal}]=0.05 \mathrm{M}$ in a salt solution of variable concentration. This system interpolates between the RHB and RHA systems and does not appear to have been studied before. The composition of the samples is summarised in table 5.1.

All rheometry measurements described here were carried out on either a stress controlled Thermal Analysis (TA) AR2000 rheometer (operated in strain controlled mode using feedback) using a 2 deg $40 \mathrm{~mm}$ diameter acrylic cone and steel plate or strain controlled Rheometric ARES rheometer using a $2.3 \mathrm{deg} 50 \mathrm{~mm}$ diameter polysulphide cone and steel plate at $T=22^{\circ} \mathrm{C}$ unless noted otherwise. Ideally, to avoid possible feedback issues, the strain controlled rheometer would have been used for all work here - unfortunately, the instrument was broken most of the time. All samples were stored at $T=22^{\circ} \mathrm{C}$ and were measured within 3-14 days of preparation.

\subsection{Measurements and analysis}

\subsubsection{Linear rheology}

The strain amplitude dependence of $G^{\prime}(\omega)$ and $G^{\prime \prime}(\omega)$ was established for selected samples. Typical results are shown in Fig. 5.1. Above a strain amplitude of $\gamma_{0} \approx 1$ the output stress is no longer independent of the input strain. This regime must be avoided in linear rheology. On the basis of these measurements, a strain amplitude of $\gamma_{0}=0.02=2 \%$ was conservatively chosen for all measurements described here.

The measured frequency response of the RHB, RHA and RHC samples is shown in 

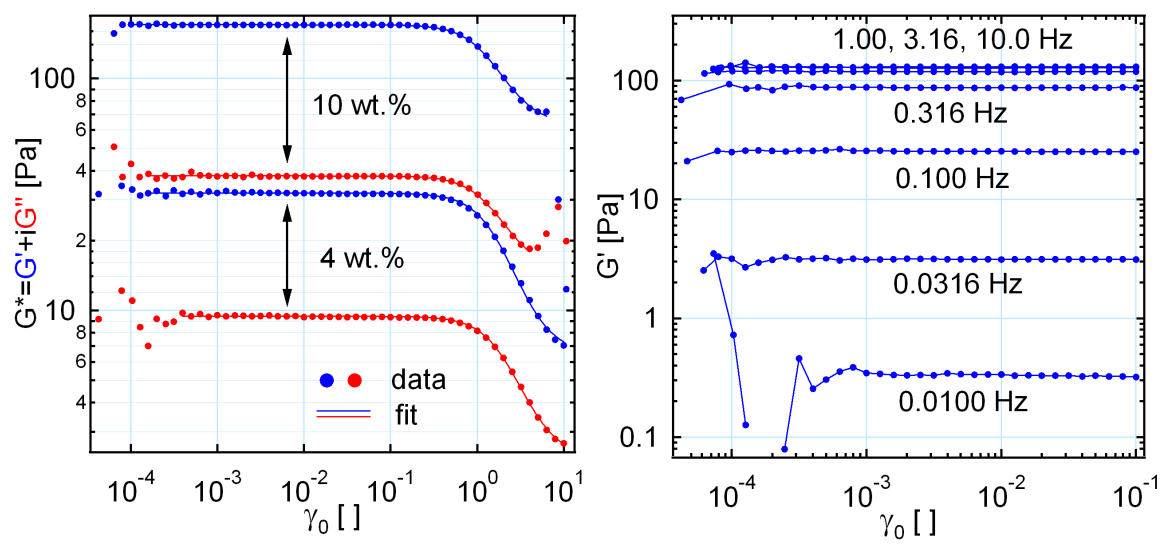

Figure 5.1: Strain amplitude dependence of $\phi=4$ and 10 wt.\% RHB samples.

Fig. 5.2 i, iii and v. The data were fitted to the frequency response of a Maxwell fluid

$$
\begin{aligned}
G^{\prime}(\omega) & =G_{M} \frac{\omega^{2} \tau_{M}^{2}}{1+\omega^{2} \tau_{M}^{2}}, \\
G^{\prime \prime}(\omega) & =G_{M} \frac{\omega \tau_{M}}{1+\omega^{2} \tau_{M}^{2}},
\end{aligned}
$$

up to the $G^{\prime}(\omega)=G^{\prime \prime}(\omega)$ crossover to determine the characteristic Maxwell time $\tau_{M}$ and amplitude $G_{M}$. Fitted parameters are shown in Fig. 5.2 ii, iv and vi. $\tau_{M}$ and $G_{M}$ are correlated with the weight fraction or salt concentration in the RHB and RHC samples. This correlation is not observed in the RHA sample. In this sample, $G_{M}$ is approximately constant at intermediate NaSal concentrations whereas $\tau_{M}$ shows two peaks - one centred on $[\mathrm{NaSal}]=0.065 \mathrm{M}$ and a second centred on $[\mathrm{NaSal}]=0.3 \mathrm{M}$.

The experimental $G^{*}(\omega)$ data were divided by $G_{M}$. These normalised data are shown in Fig. 5.2 on a parametric Cole-Cole plot along with the behavior of a Maxwell fluid calculated according to Eqn. 5.1 and 5.2 (in blue) with $G_{M}=1$. For clarity, the high frequency turn-up, associated with the increase in $G^{\prime \prime}(\omega)$ beyond its local minimum, has been excluded from this plot. For a Maxwell fluid, the trajectory of the $G^{\prime \prime}(t) / G_{M}$ and $G^{\prime}(t) / G_{M}$ on a Cole-Cole plot is described by a semicircle of parametric form $G^{\prime}(t) / G_{M}=$ $(\cos t+1) / 2$ and $G^{\prime \prime}(t) / G_{M}=(\sin t) / 2$ with $0<t<\pi$. The RHB sample becomes increasingly Maxwell-like with increasing concentration. Above $\phi=5 \mathrm{wt} . \%$ the normalised data are indistinguishable within experimental error. The data do not, however, converge to the semicircle predicted for a Maxwell fluid. The red data in Fig. 5.3 i were measured with the 2 deg $40 \mathrm{~mm}$ acrylic cone-plate geometry on the AR2000 rheometer. Additional measurements of the $\phi=10 \mathrm{wt} . \%$ RHB sample were made with a $1 \mathrm{deg} 40 \mathrm{~mm}$ acrylic coneplate on the AR2000 rheometer (Fig. 5.3 i 10B) and a 2.3 deg $50 \mathrm{~mm}$ polysulphide coneplate on the ARES rheometer (Fig. 5.3 i 10C). These measurements are quite comparable to the measurements made with the $2 \mathrm{deg} 40 \mathrm{~mm}$ cone-plate (Fig. 5.3 i 10A) and would 

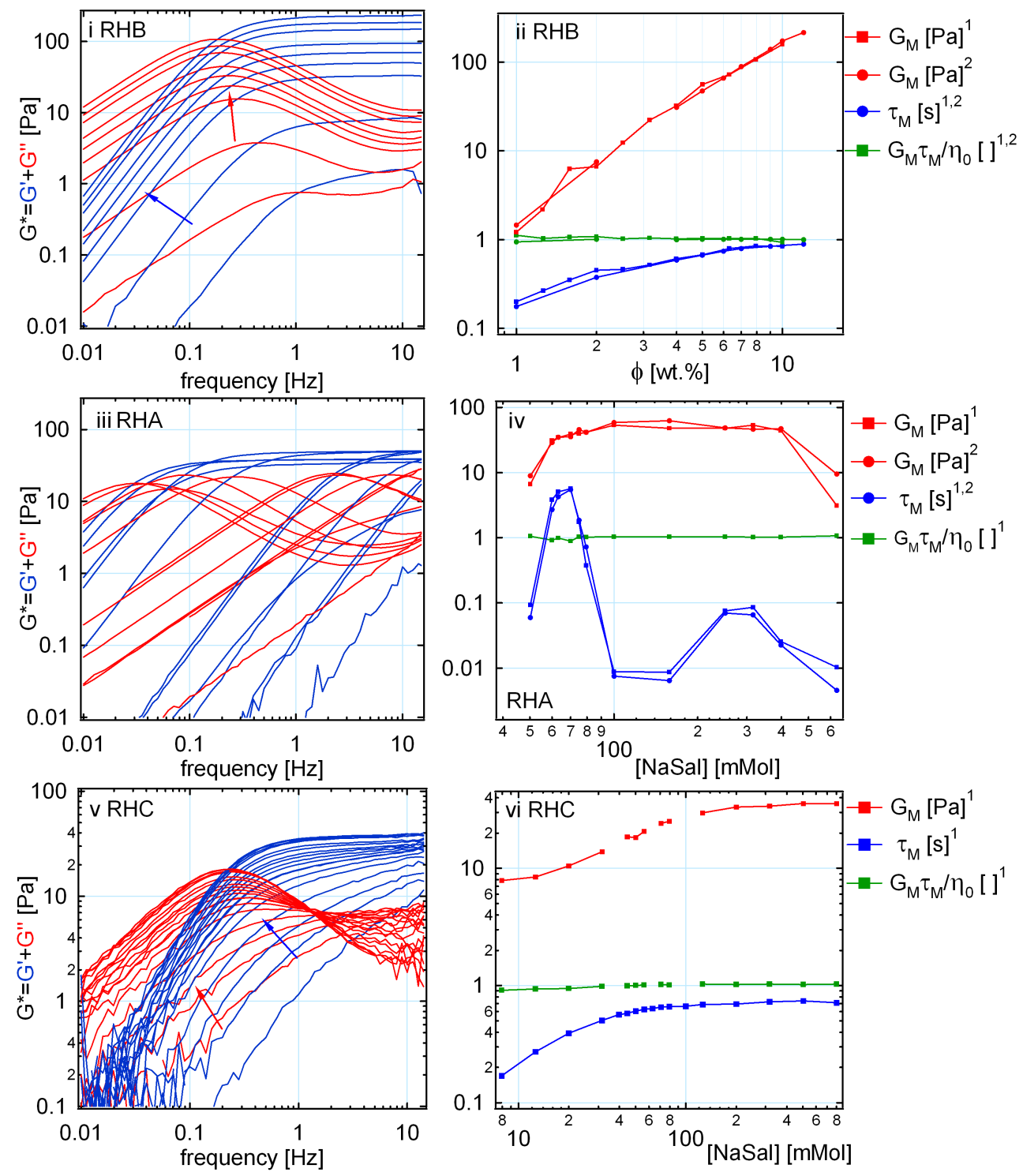

Figure 5.2: i,iii and v) Measured $G^{*}(\omega)$ for selected RHB, RHA and RHC samples with arrows indicating increasing i) $\phi$ and v) $[\mathrm{NaCl}]$. ii,iv and vi) Fitted parameters $G_{M}$ and $\tau_{M}$. The ratio $G_{M} \tau_{M} / \eta_{0} \approx 1$ was calculated from measurements of the zero shear viscosity in section 5.3.2. ${ }^{1}$ ARES $2.3 \mathrm{deg} 50 \mathrm{~mm}$ cone-plate. ${ }^{2} \mathrm{AR} 20002 \mathrm{deg} 40 \mathrm{~mm}$ cone-plate. 
suggest that the deviation from a semicircle is not an artefact of the instrumentation or associated with sample inertia. Maxwell-like behaviour is observed in some of the RHA samples; in particular, in those samples that exhibit a large $\tau_{M}$. However, a similar deviation from a semicircle is observed. The RHC sample shows a systematic change in the high frequency behavior with concentration. The normalised RHC data are superimposed on the behavior predicted by the reptation-reaction model for log-spaced value of ratio $\tau_{b} / \tau_{\text {rep }}$. These analytic data were calculated according to the Poisson-renewal model for $G_{e}=1$, fitted up to the $G^{\prime}(\omega)=G^{\prime \prime}(\omega)$ crossover and divided by the fitted $G_{M}$ to replicate the normalisation of the experimental data. Note that the normalised analytic data converge to a semicircle predicted by the Eqn. 5.1 and 5.2 when $\tau_{b} \ll \tau_{r e p}$. The experimental data loosely map onto the behaviour predicted by the reptation-reaction model. As before, the experimental data do not converge to a semicircle.

If a fluid is Maxwell-like $G_{e}=G_{M}$ and $\tau_{r}=\tau_{M}$. The terminal relaxation time $\tau_{r}$ is defined by

$$
\tau_{r}=\eta_{0} / G_{e}=\frac{1}{G_{e}} \int_{0}^{\infty} G(t) d t
$$

In the limit $\tau_{b} \ll \tau_{r e p}$ Maxwell-like behavior is predicted for wormlike micelles solutions and it is expected that $\tau_{r} \simeq \tau_{b}^{\alpha} \tau_{\text {rep }}^{\beta}$ where $\alpha$ and $\beta$ depend on the details of the reaction scheme. For example, if only breakage and recombination occurs $\alpha=\beta=1 / 2$. In this limit, $\tau_{b}$ and $\tau_{\text {rep }}$ cannot be determined separately using the reptation-reaction model. If the fluid is not Maxwell-like $G_{e} \neq G_{M}$ and $\tau_{r} \neq \tau_{M}$. In this case, it may be possible to determine $G_{e} / G_{M}$ and the ratio $\tau_{b} / \tau_{r e p}$ by comparing the measured data to analytic predictions shown in Fig. 5.3 iii and using a measurement of the zero shear viscosity to determine $\tau_{r}$. The relationship between $\tau_{b} / \tau_{\text {rep }}$ and $\tau_{b} / \tau_{r}$ is shown in Fig. $5.3 \mathrm{v}$. These data are taken directly from [108]. Using this relation and comparison above $G_{e}$, $\tau_{r}, \tau_{b}$ and $\tau_{r e p}$ can be determined separately. This analysis relies on the sample being not Maxwell-like and that the high frequency behaviour is not significantly perturbed by Rouse/breathing modes. These relaxation modes produce a turn-up at high frequencies and can obscure the behavior predicted according to the reptation-reaction model.

The deviation from the semicircle predicted for a Maxwell fluid makes the analysis above difficult. In all three cases, the data appear to converge to an ellipse of the parametric form $G^{\prime \prime}(t) / G_{M}=(\sin t) / 2$ and $G^{\prime}(t) / G_{M}=f(\cos t+1) / 2$ where the scaling factor is $f \approx 1.05$. To facilitate analysis, the experimental $G^{\prime}(t) / G_{M}$ data were rescaled by a factor $f$. The RHB and RHA measurements are still difficult to analyse using the reptation-reaction model because the Rouse/breathing mode turn-up occurs at relatively low frequencies. The RHC sample is amenable to the analysis described above. Fig. 5.3 iv shows the rescaled RHC data. The ratios $\tau_{b} / \tau_{\text {rep }}$ and $G_{e} / G_{M}$ were estimated by comparing the normalised measurements to the normalised analytic data shown in this figure. Estimated $\tau_{b} / \tau_{r e p}$ and $G_{e} / G_{M}$ are shown in Fig. 5.3 vi. Measurements of $\eta_{0}$ (described in the next section) and $G_{e}$ were used to determine $\tau_{r} . \tau_{b}$ and $\tau_{r e p}$ were determined separately using the relation described in Fig. 5.3 iv. Notably, the variation of the $G_{e}$ is smaller than that observed in $G_{M}$ - the elastic modulus is approximately constant with 

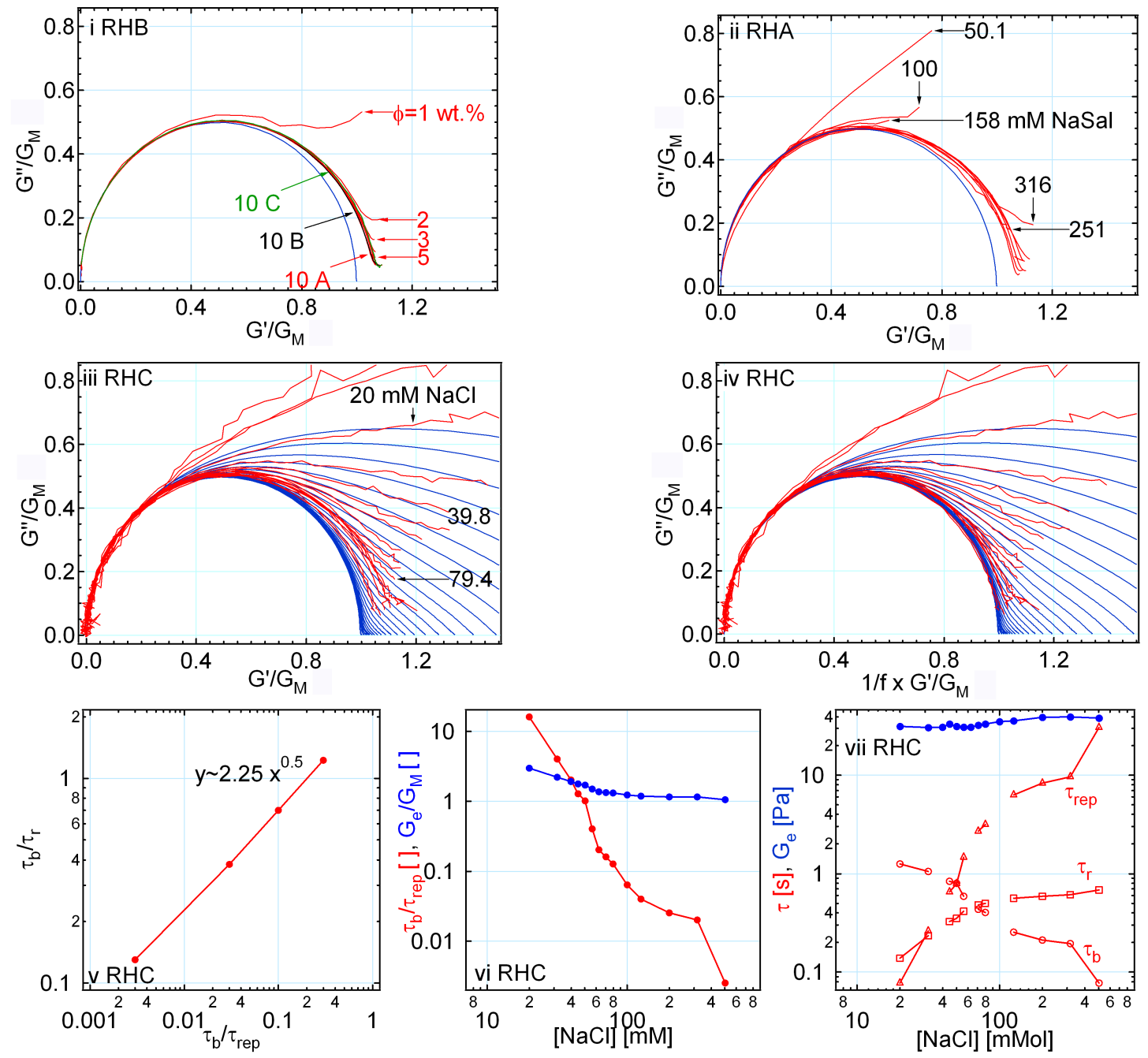

Figure 5.3: Cole-Cole plot comparing the normalised linear response of i) RHB, ii) RHA and iii) RHC samples. Convergence to a semicircle is not observed. Blue curves in iii show the behaviour predicted by the reptation-reaction model. Data in iii are rescaled in iv). The relationship between the ratio of the characteristic times $\tau_{b} / \tau_{r}$ and $\tau_{b} / \tau_{r e p}$ (from [108]) is used along with a graphical fit to $\tau_{b} / \tau_{r e p}$ from iv) to determine $\tau_{r e p}, \tau_{r}$ and $\tau_{b}$ for the RHC samples. 
changes in $\mathrm{NaCl}$ concentration. The ratio $\tau_{b} / \tau_{r e p} \propto[\mathrm{NaCl}]^{-3}$ is due to $\tau_{b}$ decreasing with $\tau_{b} \propto[\mathrm{NaCl}]^{-0.5}$ and $\tau_{r e p}$ increasing with $\tau_{r e p} \propto[\mathrm{NaCl}]^{-2.5}$

\subsubsection{Nonlinear rheology}

Measurements of the steady-state flow curves showing a time averaged 'steady-state' stress as the strain rate was stepped for the RHB, RHA and RHC wormlike micelles solutions are shown in Fig. 5.4 i, iii, v. The strain rate steps were log-spaced. The dwell time at each strain rate step for each sample was at least 2 minutes or if longer, $30 \times \tau_{M}$. The time average steady-state stress was calculated over the last $30 \mathrm{~s}$ at each strain rate step.

All samples show Newtonian behaviour with $\sigma \propto \dot{\gamma}$ at low strain rates. At intermediate strain rates, shear thinning with $\sigma \propto \dot{\gamma}^{p}, 0 \leq p<1$ is observed. A stress plateau $\sigma=$ constant is observed for a number of the samples. At high strain rates, a turn-up in stress is sometimes observed.

The zero shear viscosity $\eta_{0}$ was determined from measurements at low strain rates. If the frequency spectrum is dominated by a single relaxation mode, it is expected that $\eta_{0} \approx$ $\tau_{r} G_{e} \approx \tau_{M} G_{M}$. As a check on the fitted $\tau_{M}$ and $G_{M}$ and measured $\eta_{0}$, the ratio $G_{M} \tau_{M} / \eta_{0}$ is calculated for the data. To a good approximation it is found that $G_{M} \tau_{M} / \eta_{0} \simeq 1$ for all samples as is seen in Fig. 5.2 ii, iv and vi.

The critical stress and critical strain rate was estimated from the flow curve of samples that showed evidence of a stress plateau. This is shown in Fig. 5.4 ii, iv and vi. In [21] it was argued that the onset of the stress plateau could be expected to occur at a critical strain rate $\dot{\gamma}_{c}=2.6 / \tau_{r}$ and at critical stress $\sigma_{c}=0.67 G_{e}$ in a Maxwell-like wormlike micelles solution. $\dot{\gamma}_{c}, \tau_{c}, \sigma_{c} / G_{M}$ and $\tau_{M} \dot{\gamma}_{c}$ are shown in Fig. 5.4 ii, iv and vi in instances that a stress plateau is observed and are tabulated in Table 5.2. A stress plateau was only correlated with Maxwell-like behaviour in the RHB and RHA samples. For these samples it is found that that $\sigma_{c} / G_{M}=0.69 \pm 0.11$ and $\tau_{M} \dot{\gamma}_{c}=1.7 \pm 0.6$.

Fig. 5.5 shows the normal stress $N_{1}$ measured concurrently with the shear stress for the RHB data in Fig. 5.4 i. At low strain rates $N_{1} \propto \dot{\gamma}^{2}$ this dependence is predicted by some simple constitutive models for entangled polymer solutions and melts [5]. The normal stress continues to increase above critical stress showing approximately $N_{1} \propto \dot{\gamma}$ dependence. Perhaps not surprisingly, the dependence of the ratio $N_{1} / \dot{\gamma}$ on $\dot{\gamma}$ is very similar to the dependence of $\sigma$ on $\dot{\gamma}$.

Fig. 5.6 i and 5.6 ii show the time dependence or transient behaviour of the shear and normal stress as strain rate is stepped for an RHB and RHA sample. The flow curve is loosely divided into 6 regions. In region $\mathrm{A}$, the stress is proportional to strain rate here, the behaviour is Newtonian. In region B, shear thinning begins to occur. A stress overshoot is observed at the beginning of each step. This overshoot is observed in semidilute polymer solutions and is predicted by Eqn. 2.61. Region $\mathrm{C}$ marks the onset of the stress plateau. After an initial stress overshoot, the stress slowly drops to its equilibrium plateau value with a characteristic time that may greatly exceed $\tau_{M}$. This sigmoidal 

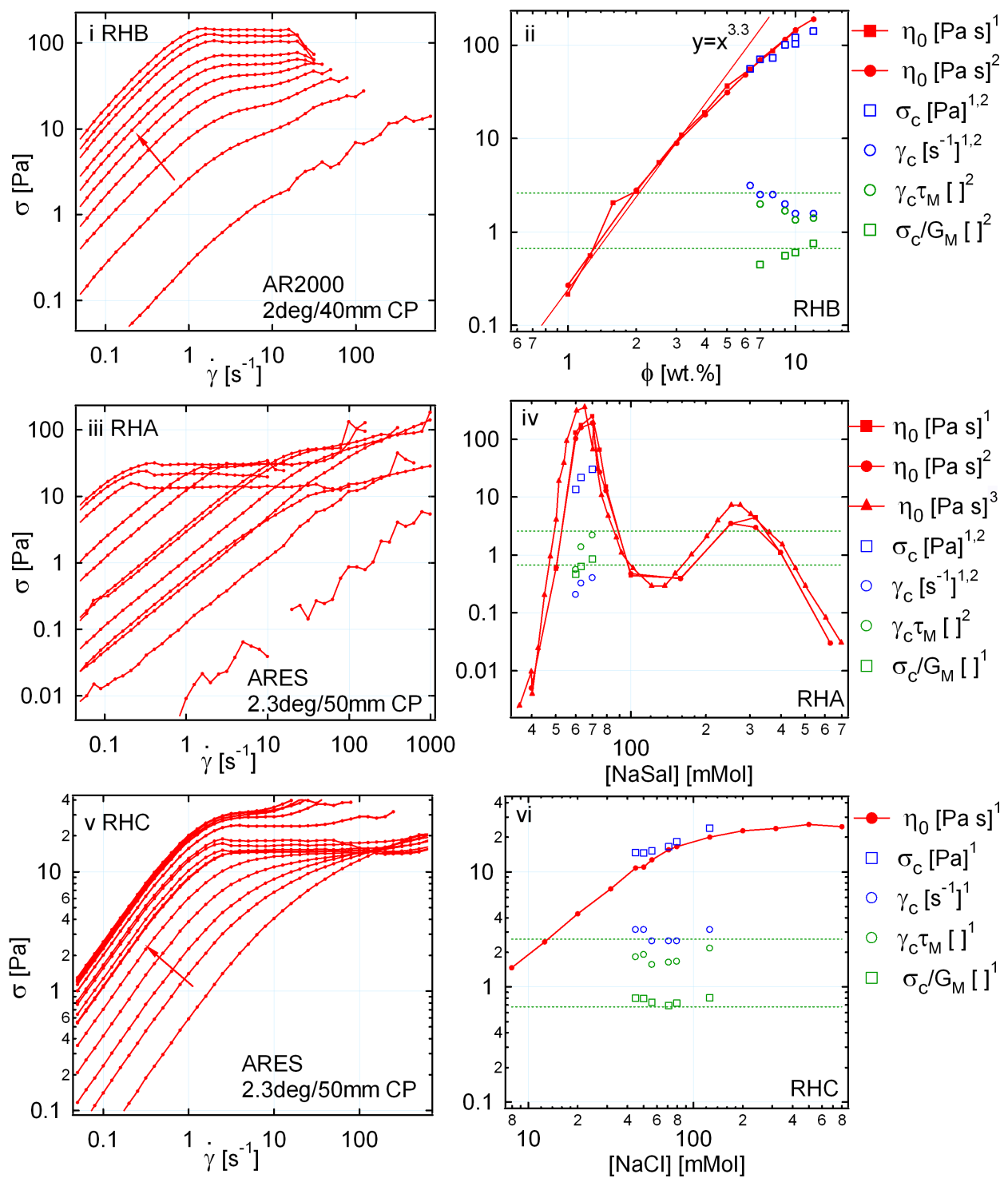

Figure 5.4: i,iii and v) Pseudo steady-state flow curves of the RHB, RHA and RHB samples. Arrows indicating increasing i) $\phi$ and v) $[\mathrm{NaCl}]$. ii, iv and vi) Fitted parameters. ${ }^{1}$ ARES 2.3deg $50 \mathrm{~mm}$ cone-plate. ${ }^{2}$ ARES 2 deg $40 \mathrm{~mm}$ cone-plate. ${ }^{3}$ Extracted from [93]. 


\begin{tabular}{|l|l|l|l|l|l|l|l|}
\hline system & conditions & $G_{M}[\mathrm{~Pa}]$ & $\tau_{M}\left[\mathrm{~s}^{-1}\right]$ & $\sigma_{c}[\mathrm{~Pa}]$ & $\dot{\gamma}_{c}\left[\mathrm{~s}^{-1}\right]$ & $\sigma_{c} / G_{M}$ & $\tau_{M} \dot{\gamma}_{c}$ \\
\hline RHB & 6.31 wt. $\%$ & 79.6 & 0.786 & 56 & 3.16 & 0.7 & 2.5 \\
& 7.94 wt. $\%$ & 107 & 0.847 & 73 & 2.51 & 0.68 & 2.1 \\
& 10 wt.\% & 158 & 0.856 & 104 & 1.58 & 0.66 & 1.4 \\
& 7 wt.\% & 88.7 & 0.792 & 71 & 2.51 & 0.8 & 2.0 \\
& 9 wt. $\%$ & 138 & 0.837 & 101 & 2.00 & 0.73 & 1.7 \\
& 10 wt. $\%$ & 172 & 0.838 & 121 & 1.58 & 0.7 & 1.3 \\
& 12 wt.\% & 213 & 0.89 & 141 & 1.58 & 0.66 & 1.4 \\
\hline RHA & $60 \mathrm{mM}$ & 29.1 & 2.71 & 13.7 & 0.208 & 0.47 & 0.56 \\
& $63.1 \mathrm{mM}$ & 34.7 & 4.25 & 22 & 0.323 & 0.63 & 1.4 \\
& $70 \mathrm{mM}$ & 35.4 & 5.44 & 30.3 & 0.405 & 0.86 & 2.2 \\
\hline RHC & $44.6 \mathrm{mM}$ & $18.6(33)$ & $0.578(0.33)$ & 18.8 & 3.16 & $1.0(0.57)$ & $1.8(1.0)$ \\
& $50.1 \mathrm{mM}$ & $18.4(31)$ & $0.605(0.35)$ & 14.6 & 3.16 & $0.79(0.47)$ & $1.9(1.1)$ \\
& $56.2 \mathrm{mM}$ & $20.7(31)$ & $0.624(0.41)$ & 15.2 & 2.51 & $0.73(0.49)$ & $1.6(1.0)$ \\
& $70.8 \mathrm{mM}$ & $24.1(32)$ & $0.653(0.48)$ & 16.6 & 2.51 & $0.69(0.51)$ & $1.6(1.2)$ \\
& $79.4 \mathrm{mM}$ & $25.3(33)$ & $0.662(0.50)$ & 18.3 & 2.51 & $0.72(0.55)$ & $1.7(1.3)$ \\
& $126 \mathrm{mM}$ & $29.9(36)$ & $0.689(0.52)$ & 24.1 & 3.16 & $0.81(0.68)$ & $2.2(1.6)$ \\
\hline P105 & $25^{\circ} \mathrm{C}$ & 4 & 400 & 2 & 0.004 & 0.5 & 1.6 \\
\hline
\end{tabular}

Table 5.2: Critical parameters for samples showing a stress plateau. The critical stress was calculated from the average steady state stress on the plateau. The critical strain rate was assigned to the first strain rate at which a sigmoidal relaxation in stress was evident. For a given sample, the uncertainty in the critical stress and critical strain rate is conservatively estimated to be $2 \%$ and $25 \%$ respectively. Bracketed values are for $G_{M}=G_{e}$ and $\tau_{M}=\tau_{r}$. The P105 system is discussed in Chapter 6.
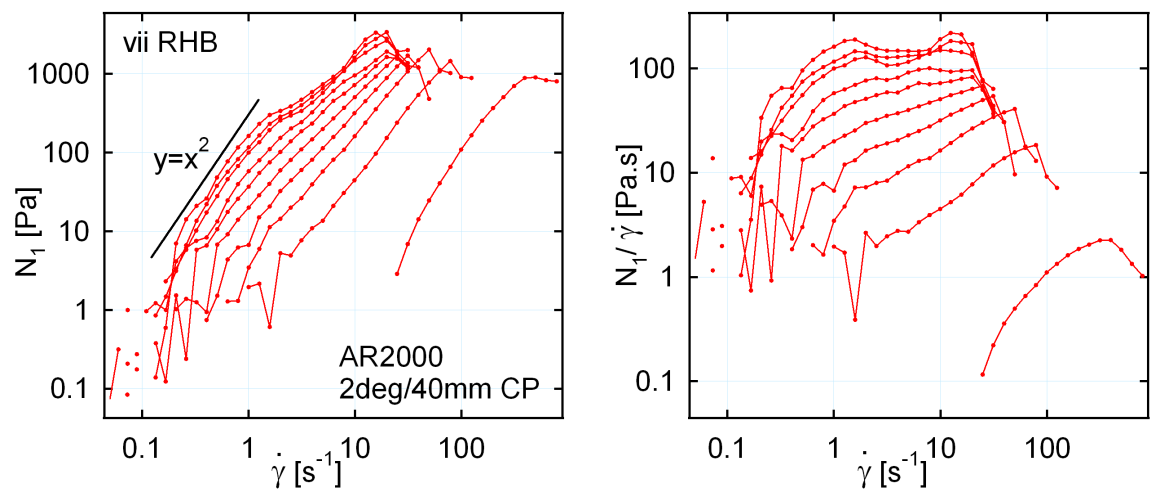

Figure 5.5: i) Normal stress difference $N_{1}$ and ii) the ratio $N_{1} / \dot{\gamma}$ for the RHB samples. 
relaxation observed was described in Chapter 3. Region D is the stress plateau. At the beginning of each strain rate step in this region, the stress overshoot is followed by a damped oscillation. A fit to the damped oscillation

$$
\sigma=\sigma_{0}+A \sin \left[\left(t-t_{0}\right) / \tau_{o}+\phi_{0}\right] \exp \left[-\left[\left(t-t_{0}\right) / \tau_{d}\right]^{p}\right]
$$

with $p=0.5$ is shown (in blue) on the right of Fig. 5.6 in several cases. Note that the damping is not monoexponential. The characteristic oscillation time $\tau_{o}$ is clearly different between the samples and decreases with increasing strain rate. This damped oscillation appears to be superimposed on low frequency fluctuations. The amplitude of these fluctuations also increases with strain rate. A slight upturn in the flow curve is observed in region E. This could mark the onset of the high strain rate branch of the flow curve. The sample is partially expelled in region F. This partial expulsion is characterised by a drop in both the shear and normal stress. Some care must be taken with the interpretation of the data in this region as significant low frequency fluctuations are often present which might be mistaken for rheo-chaotic behaviour in the sample. Pseudoperiodic fluctuations in stress in this region often appear to be correlated with the expelled sample being dragged by the edge of the geometry. Notably absent in these data on the plateau are significant stress fluctuations that might indicate the presence of rheo-chaos.

The behavior observed in the RHB and RHA samples is contrasted with that of two samples that are known to exhibit significant stress fluctuations. The response of an aqueous $[\mathrm{CTAB}]=0.05 \mathrm{M}$ and $[\mathrm{NaSal}]=0.07 \mathrm{M}$ wormlike micelles sample shown in Fig. 5.6 iii shows many similarities to the data shown in Fig. 5.6 i and ii. Towards the end of the plateau the characteristic damping time $\tau_{d}$ approaches the dwell time. In region $\mathrm{E}$ the stress begins to show oscillation of an apparently random amplitude. The normal stress does not decrease and it was confirmed by direct observation that the sample was not expelled - it appears to 'vibrate' in the gap. Large stress fluctuations are also observed in an aqueous $[\mathrm{CPCl}]=0.04 \mathrm{M}$ and $[\mathrm{NaSal}]=0.04 \mathrm{M}$ sample shown in Fig. 5.6 iv. This sample does not exhibit a clear stress plateau and unlike the sample shown in Fig. 5.6 iv oscillations are not evident. It was notable that gently rolling a volumetric flask containing the aqueous $[\mathrm{CTAB}]=0.05 \mathrm{M}$ and $[\mathrm{NaSal}]=0.07$ and the aqueous $[\mathrm{CTAB}]=0.05 \mathrm{M}$ and $[\mathrm{NaSal}]=0.07 \mathrm{M}$ between the hands induces significant turbidity in the solution. This was not observed in the RHB, RHA and RHC samples.

The measurements shown in Fig. 5.6 were made on a stress controlled rheometer operated in strain controlled mode. Strain (rate) control on a stress controlled rheometer is implemented via a feedback circuit - here the stress is dynamically adjusted to maintain a constant strain rate. Potentially, the oscillations in stress observed in Fig. 5.6 are an artefact of the feedback process between stress and strain rate. If this were the case, an oscillation in strain rate would be expected. The green curve in Fig. 5.6 iii shows the ratio $\dot{\gamma}(t) / \dot{\gamma} \times 20 . \dot{\gamma}(t)$ is the 'instantaneous' strain rate reported by the instrument at each time step and $\dot{\gamma}$ average or apparent strain at each strain rate step. Very small fluctuations are observed in this ratio. These fluctuations are approximately 300 times smaller than the ratio of the instantaneous stress to average stress around $t=5400 \mathrm{~s}$. Furthermore, there is no obvious correlation between these small fluctuations and large fluctuations in 

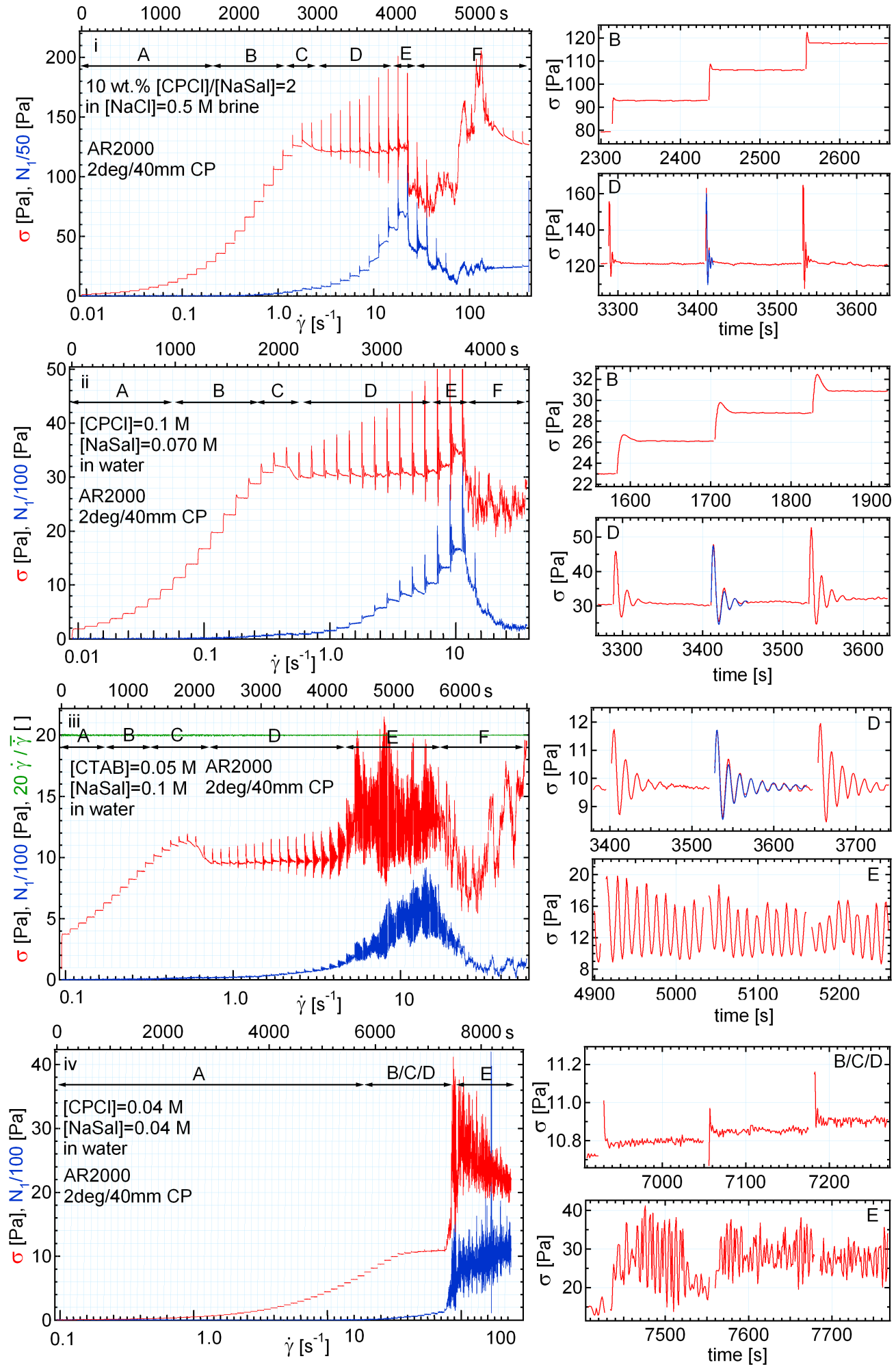

Figure 5.6: Time resolved stress during a stepped strain rate test for four wormlike micelles solutions (left). Expanded view showing the short time variation at selected points (right). 
stress. These observations seem to suggest that the feedback circuit is not significantly influencing the measured stress.

The measurements shown in Fig. 5.6 were made with a 2 minute dwell time at each strain rate step. In Fig. 5.7 i the dwell time is extended to 10 minutes to better resolve the low frequency fluctuations. Fig. 5.7 i shows time resolved log-spaced stepped strain rate measurements for a series of RHB samples of different concentrations. These data show more clearly the sigmoidal relaxation in stress at the onset of the stress plateau. This sigmoidal relaxation appears in all samples that exhibited a stress plateau. To quantify the low frequency fluctuations, the ratio of the standard deviation of the stress to average stress $\sigma_{S D} / \sigma_{\text {avg }}$ was calculated for the last 5 minutes of data acquired at each strain rate step. This ratio is shown in Fig. 5.7 ii. The ratio increases at the onset of thinning in all samples and increases with weight fraction $\phi$. Fig. 5.7 iii shows an expanded view of the stress plateau around a step in strain rate. The oscillations in stress are clearly more pronounced in the higher concentration samples. These data were fitted to Eqn. 5.4. Fitted $\tau_{o}$ is shown in Fig. 5.7 iv. In contrast to $\tau_{M}$ which increases with concentration $\tau_{o}$ decreases with increasing concentration. The green curve in Fig. 5.7 iii (and v) shows the offset stress for a $\phi=2$ wt.\% RHB sample multiplied by 20 . The cyclic variation in the stress is coherent with rotation of the experimental geometry. Notably, the period of this oscillation is significantly larger than that of the damped oscillation. If rheo-chaos was present, it would be expected that the stress would exhibit pseudo-periodic fluctuations on the plateau. Pseudo-periodicity is hinted at in Fig. 5.7 vi. A double peaked structure is observed three times (at A, B and C) in the stress measurements of the $\phi=10 \mathrm{wt} . \%$ RHB sample. Clearly this might have occurred by chance and there is no evidence of this pseudo-periodicity in the higher concentration samples, suggesting that rheo-chaos is not occurring in these samples.

Differences have been observed in the rheology of ostensibly identical samples prepared with $\mathrm{CPCl}$ from different suppliers suggesting a sensitivity to preparation and purification methods used in the production of $\mathrm{CPCl} 33$. Sensitivity to $\mathrm{CPCl}$ is investigated in Fig. 5.8. Here $\phi=8.3$ wt.\% RHB samples were prepared using $\mathrm{CPCl}$ purchased from Sigma, Arcos, Fluka and Amresco. Monohydrate and anhydrous $\mathrm{CPCl}$ are available commercially. All samples tested here were prepared with monohydrate CPCl. Samples Sigma1 and Sigma2 are two separate CPCl samples from Sigma. Sigma1 was used in the preparation of most samples used in this thesis. Sigma2a and Sigma2b were prepared with the same $\mathrm{CPCl}$ - the second sample was included to check on repeatability. Time resolved flow curves are shown in Fig. 5.8 i. The data has been offset by constant amount for clarity. The temporal fluctuations in stress are very comparable between the samples. The zero shear viscosity and critical stress for the six samples is evaluated in Fig. 5.8 ii. The critical stress for the six samples is very comparable. The zero shear viscosity is less so.

Finally, the effect of experimental flow geometry on the measured stress is investigated in Fig. 5.9 and compared to measurements made with the reference 2 deg 40 $\mathrm{mm}$ acrylic cone - steel plate geometry. Multiple samples from different suppliers were 

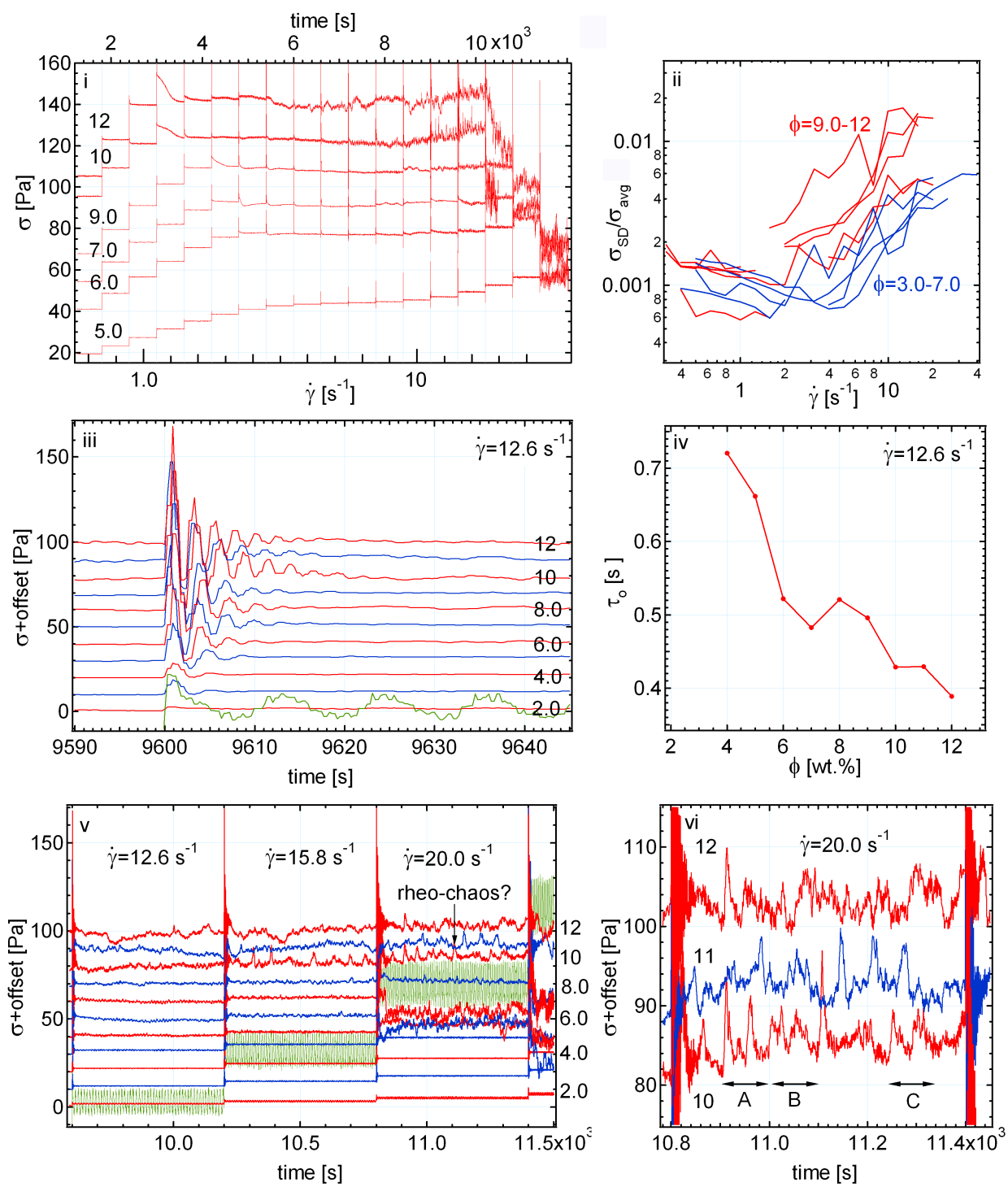

Figure 5.7: i) Time resolved stress during a stepped strain rate test with a 10 minute dwell time at each strain rate step for selected RHB samples. ii) Ratio of the standard deviation to average stress over the last 5 minutes at each strain rate step. iii) Time resolved stress following a step in strain rate showing a damped oscillation associated with a stress overshoot. iv) Characteristic oscillation time $\tau_{o}$ of the stress overshoot with concentration. Time resolved stress between v) $t=9600-11500 \mathrm{~s}$ and between vi) $t=10800-11400 \mathrm{~s}$ for selected samples. Green curves show a cyclic variation in the stress which is coherent with the rotation of the geometry. 

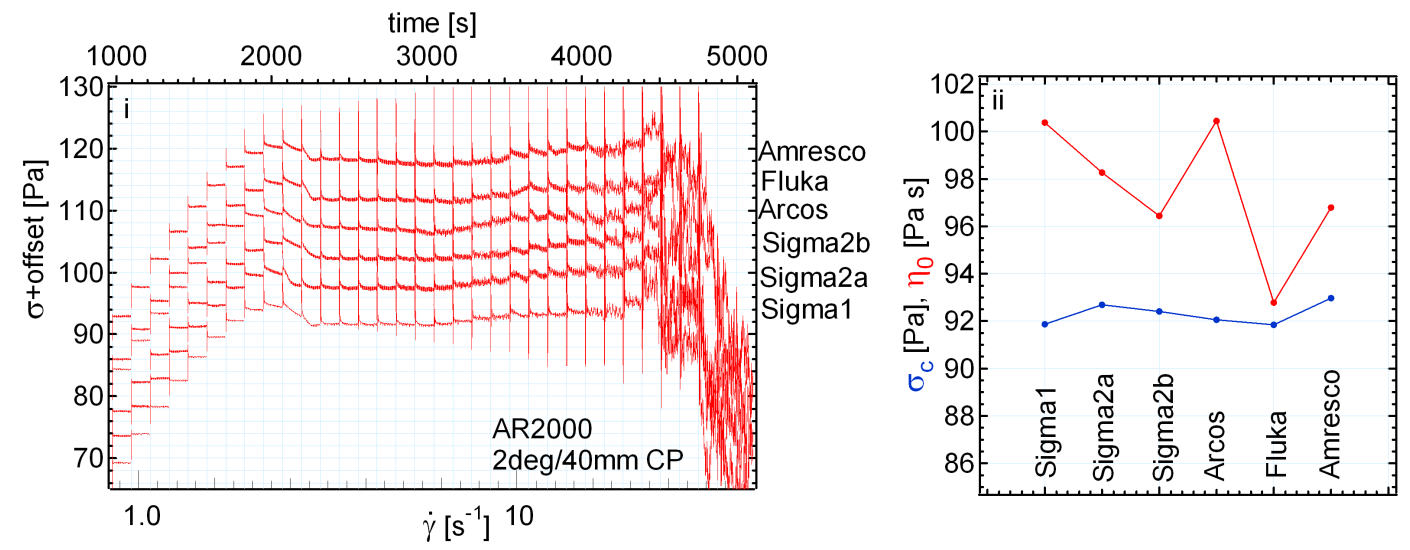

Figure 5.8: i) Time resolved stress during a stepped strain rate test comparing $\phi=8.3$ wt.\% RHB samples prepared with $\mathrm{CPCl}$ from different suppliers. Samples Sigma2a and Sigma2b are prepared from the same batch of $\mathrm{CPCl}$. ii) A comparison of the critical shear stress and the zero shear viscosity measured for the six samples.

measured to check repeatability. In Fig. 5.9 i the $\phi=8.3$ wt.\% RHB samples were measured using a $1 \mathrm{deg} 40 \mathrm{~mm}$ acrylic cone. The stress variation in this geometry is closer to that of the idealised planar-Couette geometry. Quite remarkably, at around the strain rate that the sample is expelled/fractured in the reference geometry, the samples exhibit pronounced stress fluctuations which are reminiscent of those seen in Fig. 5.6 iii. Measurements using a $2 \mathrm{deg} 60 \mathrm{~mm}$ diameter steel cone are quite similar to those made with the reference geometry. Notably, the sample is expelled/fractured at a slightly lower strain rate and a slight step in stress observed with the reference geometry on the plateau is exaggerated. This can be contrasted with the behaviour observed with a 4 deg $60 \mathrm{~mm}$ steel cone. Here, the plateau is poorly resolved and the slight step in stress observed on the plateau in the reference geometry is more pronounced. Together these measurements clearly demonstrate that cone-angle and to a lesser extent cone size is influencing flow behaviour. Measurements made with the $40 \mathrm{~mm}$ steel parallel-plate geometry are shown in Fig. 5.9 iv. Interpretation of these data is less straightforward because of the inhomogeneous strain rate expected in this geometry. It is notable that the strain rate at which the sample is expelled/fractured decreases with increasing gap size.

\subsubsection{Direct observation}

Many of the solutions examined here show a dramatic change in turbidity as temperature is lowered. This increase in turbidity can be seen in Fig. 5.10 i which shows photographs of a sample at temperatures of $T=22$ and $16{ }^{\circ} \mathrm{C}$. The sample is on a Peltier element which is painted half black to enhance contrast. This turbidity rapidly disappears on reheating to $T=22^{\circ} \mathrm{C}$. A change in turbidity is often associated with a phase change in complex fluids. 

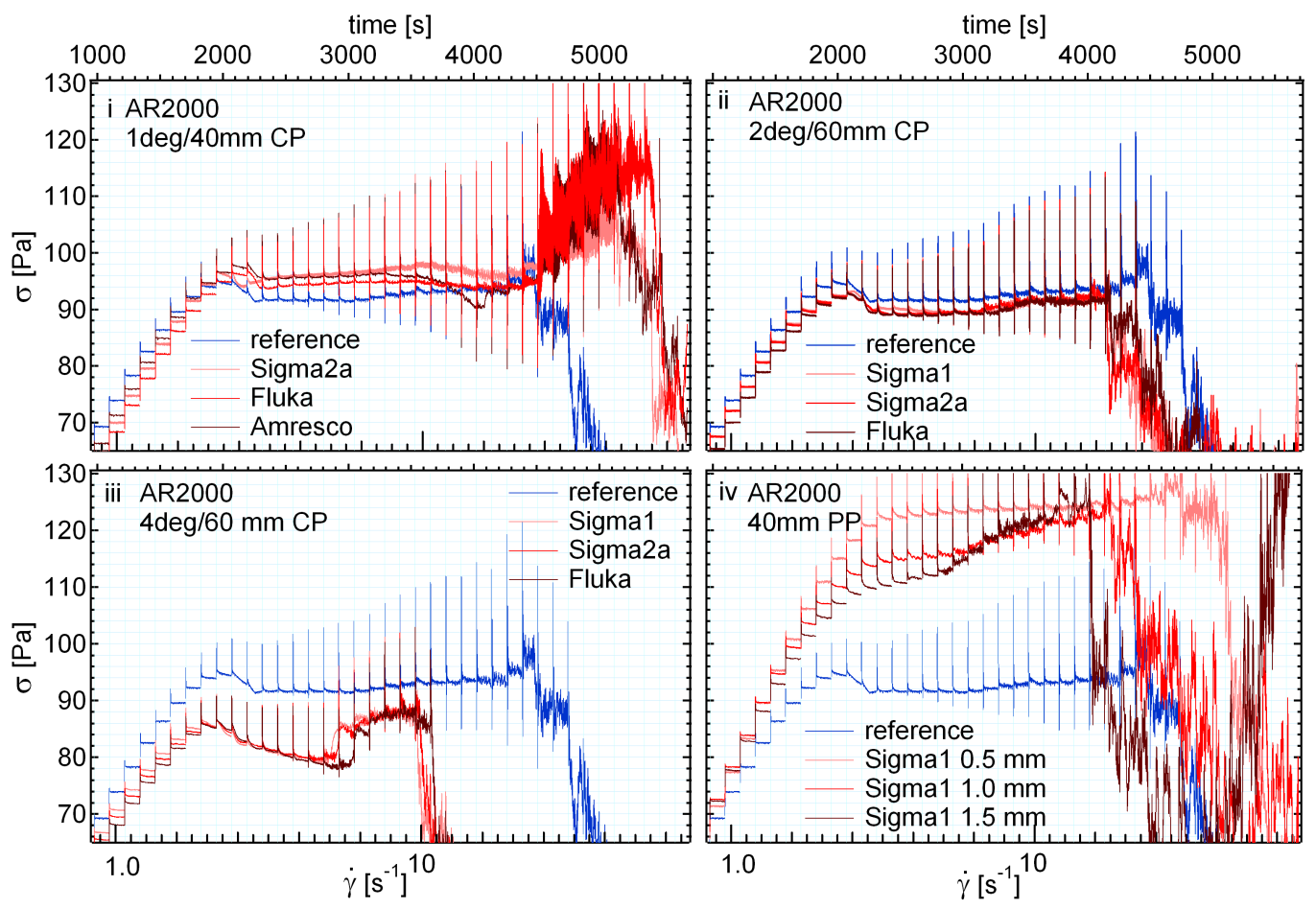

Figure 5.9: Time resolved stress during a stepped strain rate test comparing $\phi=8.3$ wt.\% RHB samples in different factory i) $1 \mathrm{deg} 40 \mathrm{~mm}$ acrylic cone - steel plate, ii) 2 deg 60 $\mathrm{mm}$ steel cone - steel plate, iii) 4 deg 60 steel cone - steel plate and iv) $40 \mathrm{~mm}$ steel plate - steel plate flow geometries. The blue curve shows measurements made with a 2 deg 40 mm acrylic cone - steel plate 'reference' geometry. 

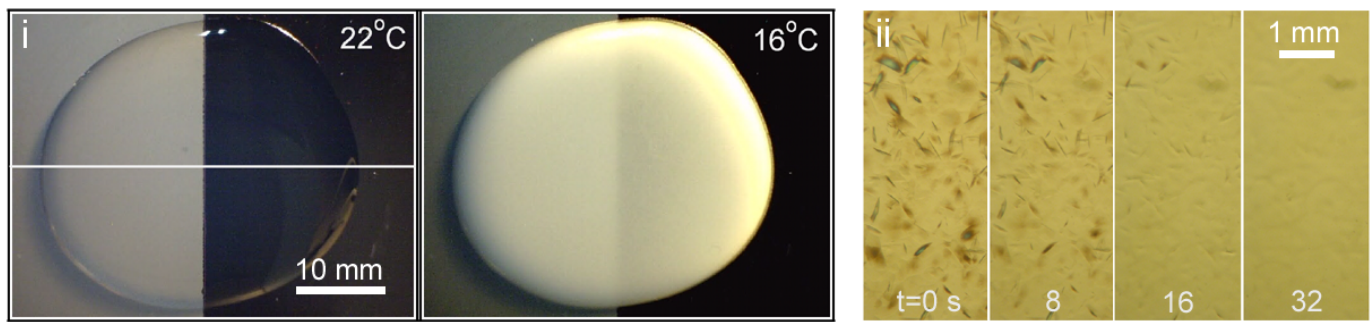

Figure 5.10: i) Photographs of a $\phi=8.3$ wt.\% RHB sample on a partly blackened Peltier plate at $T=22$ and $16{ }^{\circ} \mathrm{C}$. ii) Transmission light microscope images of a refrigerated $\phi=11$ wt.\%. RHB sample on heating to room temperature.

The light microscope study shown in Fig. 5.10 ii indicates that a crystalline precipitate forms on cooling - a phase change is indeed occurring.

Not surprisingly, this phase change is correlated with significant changes in the mechanical properties of the solution. This is demonstrated in Fig. 5.11 i-ii where both the linear response and visual appearance of the $\phi=8.3 \mathrm{wt} \%$ sample is observed as temperature is stepped between $T=22$ and 18,22 and 16 or 22 and $14{ }^{\circ} \mathrm{C}$ over $400 \mathrm{~s}$. $G^{*}(\omega)$ was monitored at a frequency of $f=1 \mathrm{~Hz}$ and the sample was photographed at $1 \mathrm{~s}$ intervals. Fig. 5.11 ii shows the image intensity over the line indicated in 5.10 (left) with time. Clouding correlates with changes in $G^{*}(\omega)$. Note that the sample rapidly recovers its initial rheology on reheating to $T=22{ }^{\circ} \mathrm{C}$. Changes in the mechanical properties of the fluid were used to determine the temperature at which crystallisation occurs in the RHB, RHA and RHC samples. This phase transition is shown in Fig. 5.11 iii-v and was determined from measurements of $G^{\prime}(\omega)$ and $G^{\prime \prime}(\omega)$ at $1 \mathrm{~Hz}$ as the temperature was ramped down at $d T / d t=-1{ }^{\circ} \mathrm{C} /$ minute from $T=25{ }^{\circ} \mathrm{C}$. The transition temperature appears to be fairly well correlated with $\tau_{M}$. This correlation is not perfect however - the second peak present in $\tau_{M}$ at high NaSal concentration is not reflected in the measurements of the transition temperature.

The visual behaviour of $\phi=5.0$ and 8.3 wt.\% RHB solutions under shear flow conditions were examined in homemade optical cone-plate, parallel-plate and cylindricalCouette flow geometries. The rheo-optical geometries used in this thesis were not directly temperature controlled. Instead, the sample temperature was regulated by an airconditioner/inverter located in the laboratory. This air-conditioner was able to maintain the room temperature at $22^{\circ} \mathrm{C}$ to within $0.5^{\circ} \mathrm{C}$.

In the cone-plate and parallel-plate geometries, the sample was mounted on a static transparent glass plate and illuminated from below. Shear was applied to the sample using either a transparent $4 \mathrm{deg} 60 \mathrm{~mm}$ acrylic cone or a homemade $60 \mathrm{~mm}$ glass disc with a 1.5 mm gap. The strain rate was stepped linearly in $\dot{\gamma}=0.5 \mathrm{~s}^{-1}$ steps with a dwell time of 120 $\mathrm{s}$ at each step. The shear stress was recorded continuously. The sample was photographed at $5 \mathrm{~s}$ intervals from above at a scattering angle of $\theta \approx 30 \mathrm{deg}$ with a fixed exposure time 

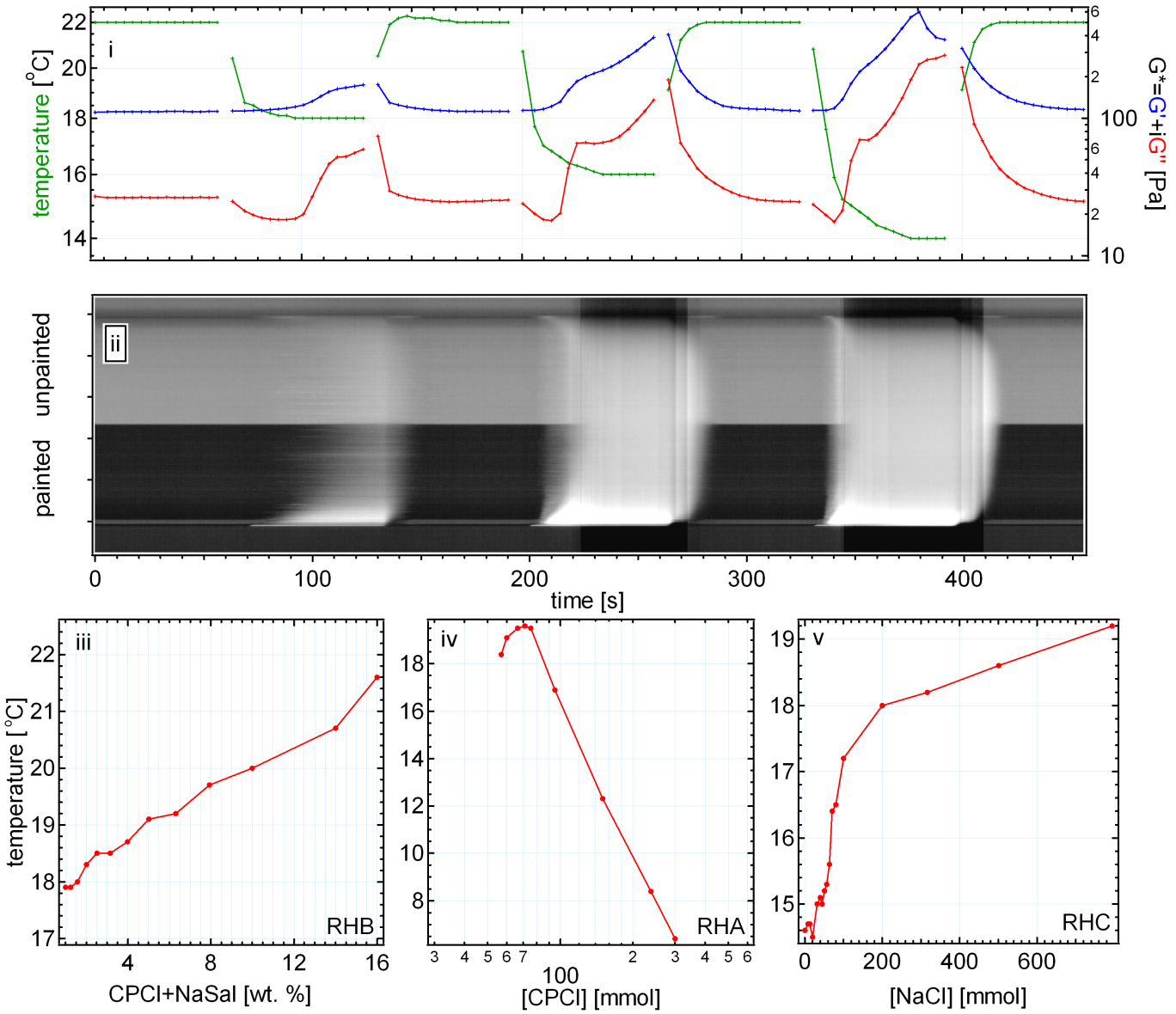

Figure 5.11: A comparison between i) mechanical and ii) visual behaviour of a 8.3 wt.\% RHB sample with temperature. Phase transition temperatures of selected iii) RHB, iv) RHA and v) RHC samples. 
of approximately $1 \mathrm{~s}$.

The measured stress with photographs at indicated strain rates are shown in Fig. 5.12 and 5.13 . As the strain rate is increased, both samples exhibit shear induced turbidity. For a 'well behaved', fluid the local strain rate is expected to be approximately constant in the cone-plate geometry and to vary radially in the parallel-plate geometry. If turbidity was correlated with strain rate, it would be expected that scattering would vary radially in both the cone-plate and parallel-plates geometries. Radial dependence in the coneplate geometry could be expected because of the radial variation in the gap size. Radial dependence in the scattered intensity is observed in the $\phi=5.0 \mathrm{wt} \% \mathrm{RHB}$ wormlike micelles solution at low strain rate in both cone-plate and parallel-plates geometries. The turbidity appears to saturate at higher strain rates. Similar behaviour is observed for the higher concentration $\phi=8.3$ wt. $\%$ RHB sample at lower strain rates. Above $\dot{\gamma}>2$ $\mathrm{s}^{-1}$ turbid rings or spirals are observed in the sample. These rings/spirals slowly migrate radially through the fluid. This shear induced turbidity almost immediately disappears on the cessation of shear. Fig. 5.12 v-vi and 5.13 v-vi show the image intensity with time over the radial line indicated in the middle photographs. Fig. $5.12 \mathrm{v}$-vi clearly demonstrates that turbidity varies smoothly with strain rate in the $\phi=5.0 \mathrm{wt} . \%$ RHB sample in both the cone-plate and parallel-plate geometries. This can be contrasted with the behaviour of the $\phi=8.3$ wt.\% RHB sample in both the cone-plate and parallel-plate geometries. Significant time-space variations in the turbidity are observed beyond $\dot{\gamma}=2 \mathrm{~s}^{-1}$. 


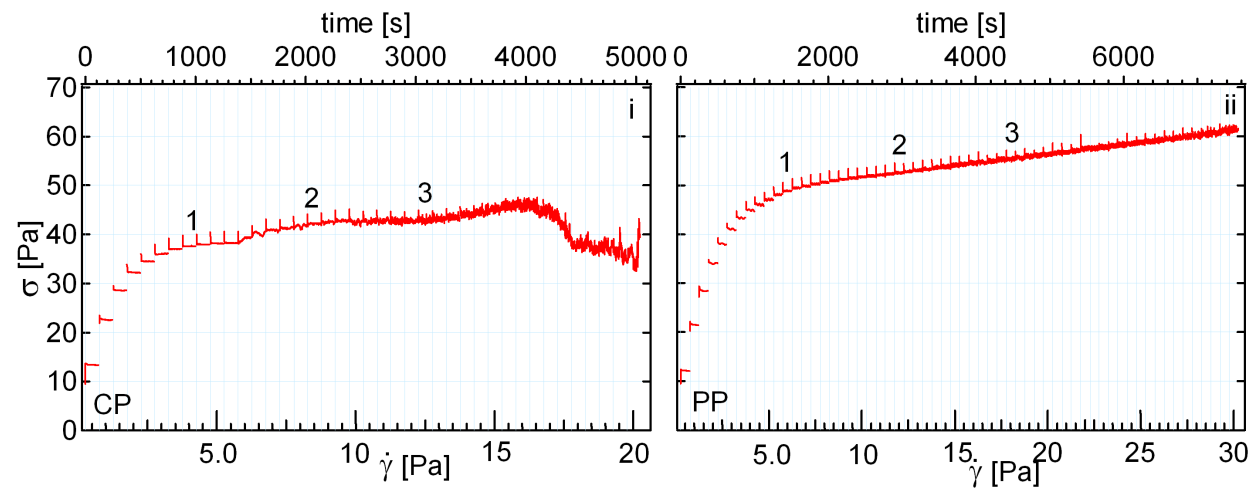

iii

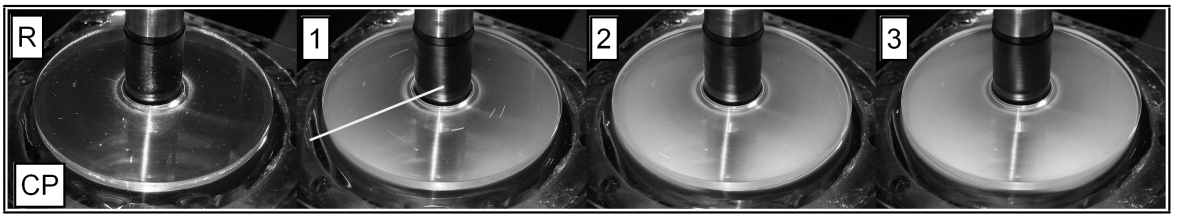

iv
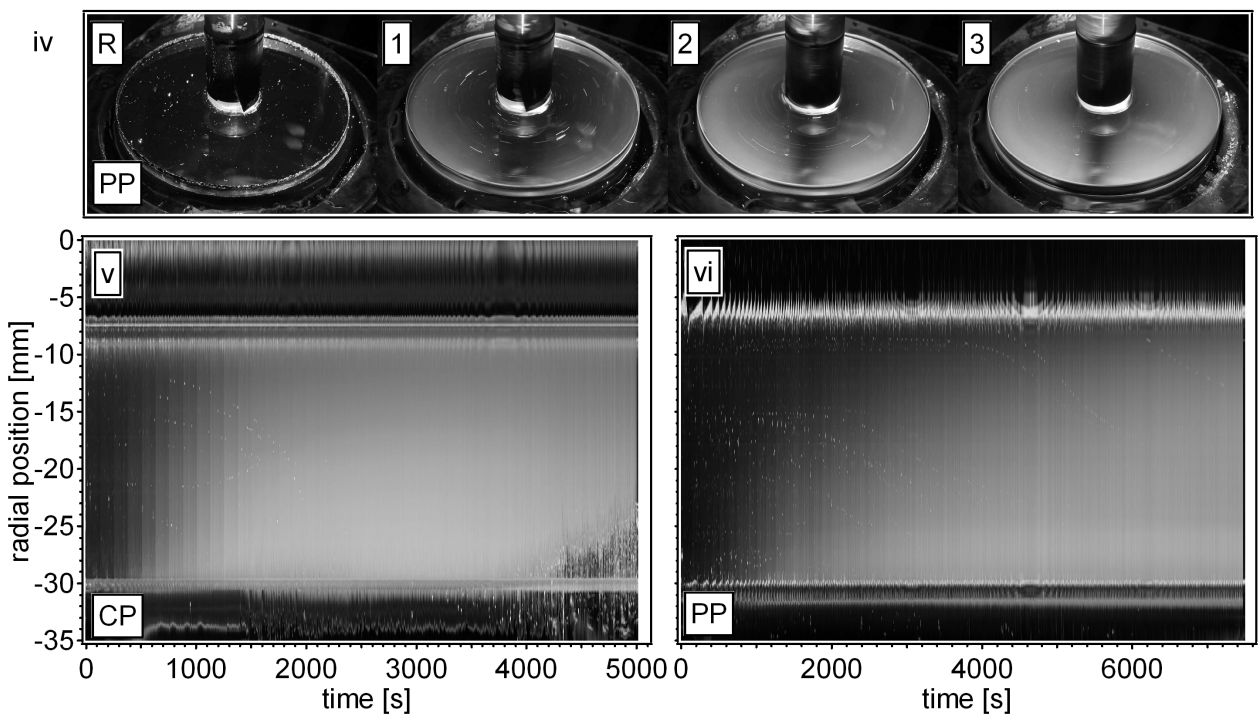

Figure 5.12: Mechanical and visual behavior of a $\phi=5.0$ wt.\% RHB sample in optical cone-plate $(\mathrm{CP})$ and optical parallel-plate $(\mathrm{PP})$ geometries during a stepped strain rate test. i-ii) Measured stress with stepped strain rate. iii-iv) Photographs of the sample at points indicated on the flow curve. v-vi) Average image intensity across the line shown with time/strain rate. 

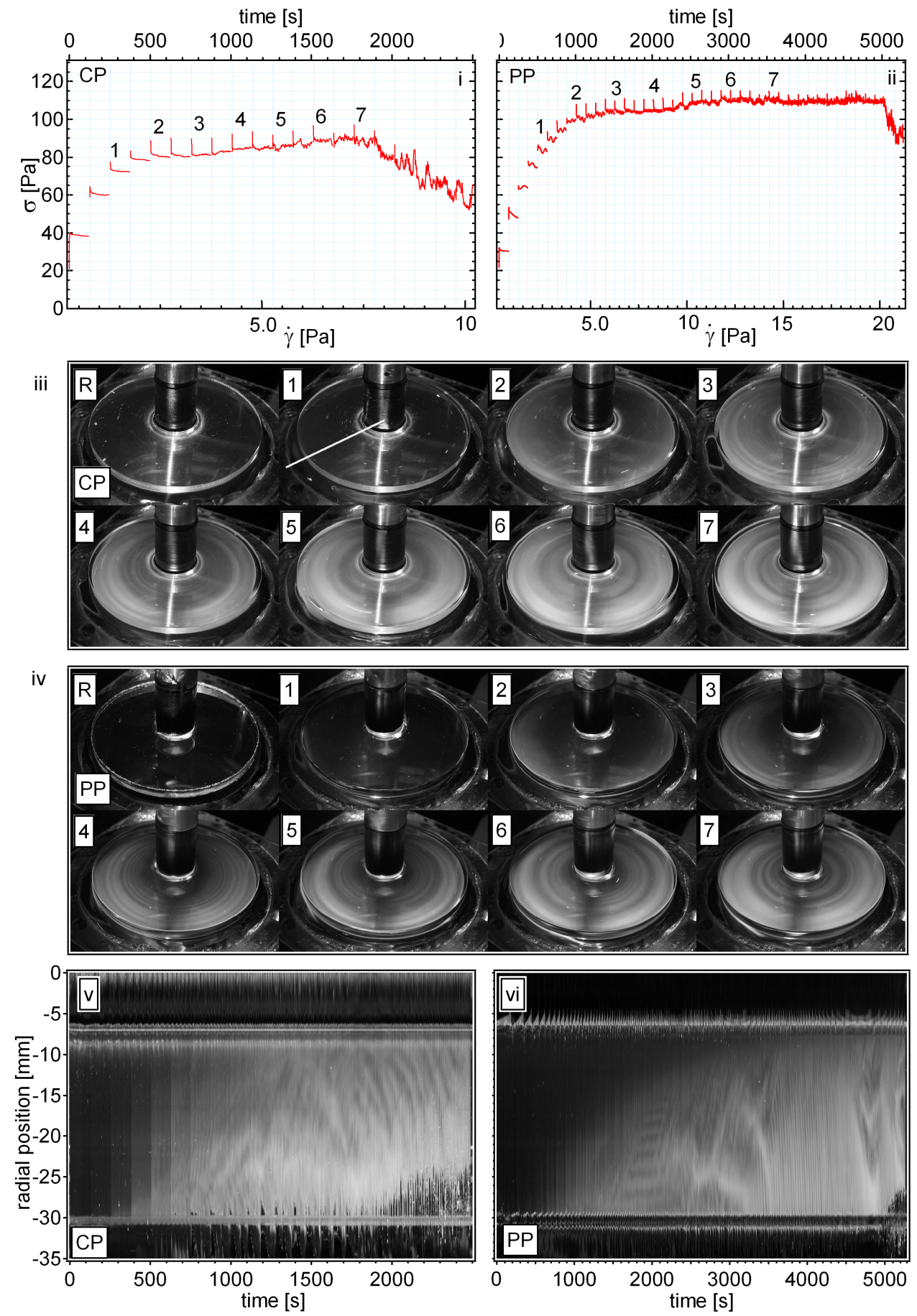

Figure 5.13: Mechanical and visual behavior of a $\phi=8.3$ wt.\% RHB sample in optical cone-plate $(\mathrm{CP})$ and optical parallel-plate $(\mathrm{PP})$ geometries during a stepped strain rate test. i-ii) Measured stress with stepped strain rate. iii-iv) Photographs of the sample at points indicated on the flow curve. v-vi) Average image intensity across the line shown with time/strain rate. 


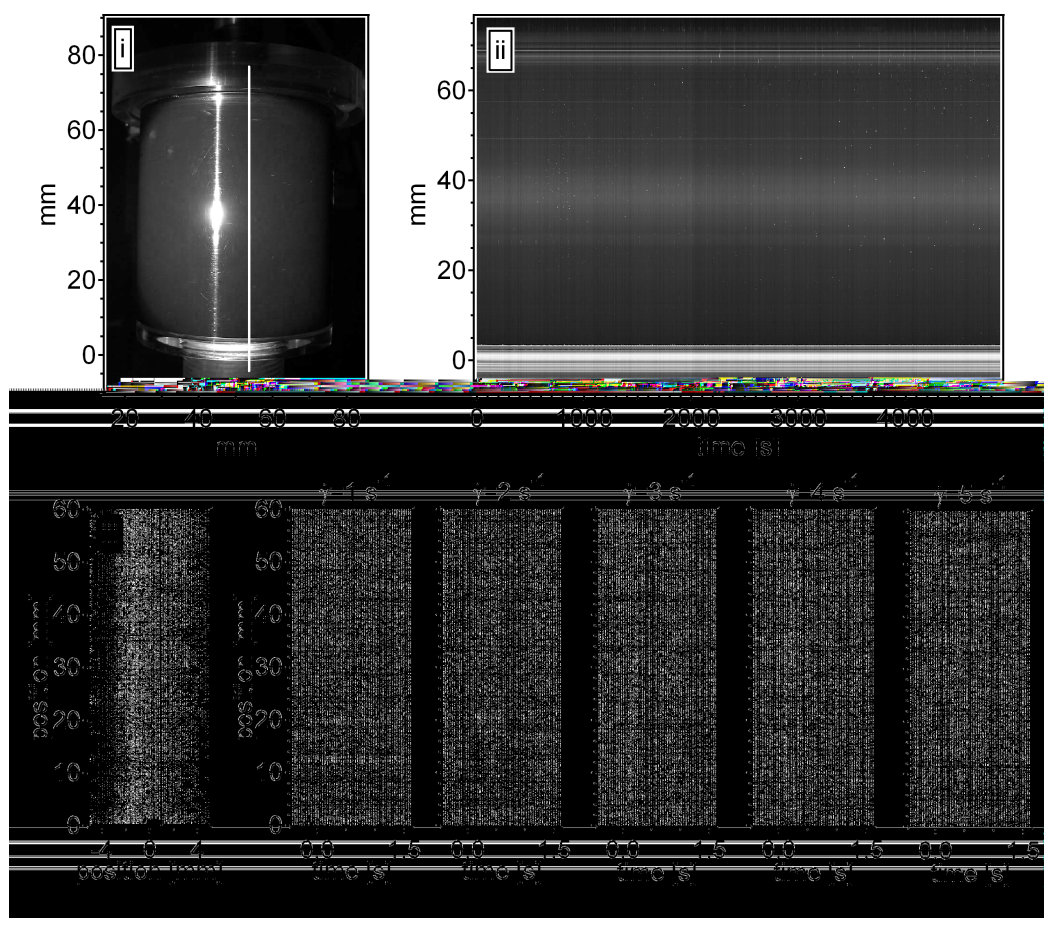

Figure 5.14: i-ii) Visual behavior of a sheared $\phi=8.3$ wt.\% RHB sample in an optical cylindrical-Couette geometry during a stepped shear rate test. iii) Image intensity over the vertical line shown on the left with time showing the formation of turbid rings along the vorticity axis when the strain rate is stepped from $\dot{\gamma}=0$ to the strain rate indicated. 
In the optical cylindrical-Couette geometry, the sample was trapped between the surfaces of a $r_{o}=25 \mathrm{~mm}$ Perspex cup and a blackened aluminium $r_{i}=24 \mathrm{~mm}$ bob. The sample was illuminated through the side wall of the cup and photographed at $5 \mathrm{~s}$ intervals as the strain rate was stepped as above. Typical results are shown in Fig. 5.14 i for an 8.3 wt.\% sample. No evidence of shear induced turbidity is seen here. Fig. 5.14 i and ii are included to demonstrate that careful illumination is required to observe this induced turbidity. In Fig. 5.14 ii an expanded laser beam was directed up the vorticity axis and the sample was filmed through the side wall of the cylindrical-Couette using a highdefinition camera. The strain rate was stepped between $\dot{\gamma}=0-1-0-2-0-3-0-4-0-5 \mathrm{~s}^{-1}$ with a dwell time of 1 minute at each strain rate step. Directly after the step to $\dot{\gamma}=2,3,4$ and 5 $\mathrm{s}^{-1}$ a series of turbid rings formed along the vorticity axis. These rings rapidly propagated up the vorticity axis before dissipating. The time evolution of the rings is shown in Fig. 5.14 iii (right). These rings may be connected with the transient oscillation seen in stress following a step in strain rate discussed in the last section. As seen in Fig. 3.8 vi [45] a turbid band of variable width was seen to form at the inner wall in a $\mathrm{CTAB} / \mathrm{NaNO}_{3}$ wormlike micelles solution sheared in a cylindrical-Couette geometry. In contrast to the rings observed here, this variable width turbid band [45] did not appear to dissipate with continued shearing.

\subsection{Discussion}

The slight deviation from the behaviour predicted by the reptation-reaction model in the fast break limit appears to be quite common although not widely acknowledged in the literature. This deviation is easy to overlook if the entire ellipse is fitted to a semicircle, suggesting that the reptation-reaction model needs slight modification.

$\tau_{b}$ and $\tau_{r e p}$ were not determined separately for the RHB and RHA using the reptationreaction model. In both the RHB and RHA samples the Rouse/breathing modes obscure the high frequency behaviour predicted by the reptation-reaction model. The effect of these modes on the rheology of the RHB and RHA samples will be examined in the next chapter.

This experimental $\sigma_{c} / G_{M}$ is very comparable to the value $\sigma_{c} / G_{e}=0.67$ expected according to the reptation-reaction model. The experimental $\dot{\gamma}_{c} \tau_{M}$ are consistently lower than the predicted values. $\dot{\gamma}_{c}$ is difficult to estimate from the data - this difference may be due to a systematic underestimation of $\dot{\gamma}_{c}$. On the basis of the RHB and RHA measurements, it might be concluded that a stress plateau is inevitable if the sample is sufficiently Maxwell-like. The RHC measurements demonstrate that this is not the case. A stress plateau is observed at intermediate concentrations. At higher concentrations, the system is more Maxwell-like but does not show a stress plateau.

The fluctuations in shear stress observed in the RHB, RHA samples in Fig. 5.6 samples show qualitative differences to the fluctuations seen in the aqueous $[\mathrm{CTAB}]=0.05$ $\mathrm{M}$ and $[\mathrm{NaSal}]=0.07$ suggesting qualitative differences in the underlying mechanical prop- 
erties of these fluids. The fluctuations seen in Fig. 5.6 iii are similar to those seen in Fig. 5.9 i indicating a sensitivity to experimental flow geometry and that some care must be taken with inferring underlying mechanical behaviour from the measured flow curve. That the samples measured in the parallel-plate geometry fractured at a lower strain rate with larger gap sizes suggests that edge effects play a role in determining flow stability of the fluid.

Turbid rings were observed in an aqueous $[\mathrm{CPCl}]=[\mathrm{NaSal}]=40 \mathrm{mMol}$ solution in both the cone-plate and parallel-plate geometries in [109]. As demonstrated by Fig. 5.6 iv this system is unlike the RHB, RHA and RHC systems in that it does not show a clear stress plateau and shear thickens. It has been demonstrated here that turbid rings also form in systems that exhibit a stress plateau.

The correlation between shear thinning and turbidity here suggests that the two are in some way connected. Turbid rings were observed in the $\phi=8.3 \mathrm{wt} . \%$ RHB sample in both the cone-plate and parallel-plate geometries. Rings were not observed in the $\phi=5.0$ wt.\% sample. From measurements made in the reference geometry the $\phi=8.3$ wt. $\%$ showed a stress plateau whereas the $\phi=5.0 \mathrm{wt} . \%$ did not. Turbid rings were observed to stack along the vorticity axis of the cylindrical-Couette geometry with the region of highest turbidity nearest the moving inner wall. The vorticity axis is directed radially in both the cone-plate and parallel-plate geometry - this space-time variation is reminiscent of the behavior illustrated in Fig. 5.13 v-vi. 


\section{Chapter 6}

\section{An experimental test of the Poisson-renewal model}

\subsection{Introduction}

The validity of the reptation-reaction model, which describes the low frequency linear rheology of wormlike micelles solutions, was examined using mechanical rheometry in Chapter 5. The reptation-reaction model describes the low frequency linear rheology of wormlike micelles solutions. The Poisson-renewal model extends the reptation-reaction model to include the influence of high frequency relaxation modes on the linear rheology. Historically, this model has been difficult to test because of the mechanical limitations of conventional rheometry. In recent years, a new technique called microrheology has been developed in which bulk rheological properties are inferred from the motion of microscopic probe particles embedded in the fluid. The upper frequency limit of this technique typically exceeds that of conventional rheometry by 3-4 decades making it a potentially useful method for testing the Poisson-renewal model.

In this chapter microrheology is introduced. Dynamic light scattering based microrheology along with conventional mechanical rheometry is used to examine the linear rheology of selected wormlike micelles solutions. These measurements are compared to the behaviour predicted by the Poisson-renewal model. Finally, the feasibility of a diffusion NMR based microrheology technique is considered.

\subsection{Microrheology}

According to the Fluctuation-Dissipation theorem, the mean square displacement $\left\langle\Delta r^{2}(t)\right\rangle$ can be expressed in terms of the inverse Fourier transform of the mechanical impedance $Z(\omega)$. Alternatively, the impedance can be expressed in terms of the Fourier transform $(\mathcal{F})$ of the mean square displacement (MSD). As noted earlier $\eta^{*}(\omega)$ is the mechanical 
impedance of a complex fluid implying that

$$
\eta^{*}(\omega)=\left[\frac{k_{B} T}{\pi a \omega^{2} \mathcal{F}\left[\left\langle\Delta r^{2}(t)\right\rangle\right]}\right]
$$

or

$$
G^{*}(\omega)=\left[\frac{k_{B} T}{i \pi a \omega \mathcal{F}\left[\left\langle\Delta r^{2}(t)\right\rangle\right]}\right]
$$

Eqn. 6.1 or 6.2 are known as the Generalised Stokes-Einstein relation [110].

In order to determine $G^{*}(\omega)$ the Fourier transform of $\left\langle\Delta r^{2}(t)\right\rangle$ must be calculated. Dasgupta and co-workers outlined a method for inverting the MSD to determine $G^{*}(\omega)$ in reference [110]. The method assumes that the MSD exhibits power law behaviour locally. With this assumption $G^{*}(\omega)$ can be written

$$
G^{*}(\omega)=G(\omega) \exp [i \pi \alpha(\omega) / 2]
$$

where

$$
G(\omega)=\frac{k_{B} T}{\left\langle\Delta r^{2}(1 / \omega)\right\rangle \Gamma[1-\alpha(\omega)]}
$$

and

$$
\alpha(\omega)=\frac{\partial \ln \left\langle\Delta r^{2}(\tau)\right\rangle}{\partial \ln \tau}
$$

In Eqn. 6.4. $\left\langle\Delta r^{2}(1 / \omega)\right\rangle$ is the magnitude of $\left\langle\Delta r^{2}(\tau)\right\rangle$ evaluated at $\tau=1 / \omega$

Eqn. 6.2 relates the bulk rheological parameter $G^{*}(\omega)$ of a host medium to the MSD of an immersed probe. A measurement of the MSD of the probe can therefore be used to determine $G^{*}(\omega)$. In Eqn. 6.2 it is implicitly assumed that the host medium is continuous. However, complex fluids exhibit heterogeneity or granularity on small length scales. Eqn. 6.2 is only valid when the dimensions of the probe are much larger than the granularity of the host medium.

Because Brownian motion is most readily observed in microscopic particles, microscope probes are commonly used. Determination of the bulk rheological parameters from the Brownian motion of microscopic probes is called microrheology (some authors make a distinction between 'passive' microrheology where the probes are perturbed by Brownian forces as above and 'active' microrheology where a known force is actively applied to the microscopic probe and the resulting motion measured). As with conventional mechanical rheometry, sensitivity to $G^{*}(\omega)$ is limited at high frequency by inertia. Because the probes used in microrheology are microscopic, inertia effects only become significant at very high frequencies. As noted in Chapter 4, both photo-correlation spectroscopy (PCS) and diffusing wave spectroscopy (DWS) are dependent on a probe particle's MSD and can potentially be used for microrheology. In general, only DWS is used for reasons that will become evident. 


\subsection{Measurements and analysis}

\subsubsection{Microrheology}

RHB wormlike micelles solutions at weight fractions of $\phi_{\mathrm{CPCl}+\mathrm{NaSal}}=2.51,3.16,3.98$ and $5.01 \mathrm{wt} . \%$ doped with approximately $1.0 \mathrm{wt} . \% 1.0 \mu \mathrm{m}$ diameter latex sphere (Polyscience) were examined using DWS microrheology. The latex spheres were provided as an aqueous suspension which itself contains a surfactant to prevent aggregation. The spheres were 'washed' before use to remove these dissolved additives by repeatedly centrifuging, decanting and diluting the commercial suspension. RHB solutions at weight fractions of $\phi_{\mathrm{CPCl}+\mathrm{NaSal}}=1.25,1.58,2.00$ and $2.51 \mathrm{wt} . \%$ doped with approximately $2 \times 10^{-5}$ wt.\% 1.0 $\mu \mathrm{m}$ latex sphere were examined using PCS microrheology.

The optical set-up for the DWS microrheology experiment is shown schematically in Fig. 6.1 i. Light from a polarised $15 \mathrm{~mW}, \lambda=633 \mathrm{~nm}$, HeNe laser (Melles Griot) was expanded using a beam expander (Edmund Optics) directed through a variable neutral density filter (Edmund Optics, not shown in illustration) and through a hole in a black acrylic stop into a $2 \mathrm{~mm}$ path length cuvette (Stana) containing the doped sample. Diffuse light transmitted through the cuvette and scattered through a $1 \mathrm{~mm}$ hole in the acrylic stop was filtered by a polariser, collected by a GRIN objective and directed by a fibre optic to a photon counting photomultiplier (PMT) (correlator.com). The hole in the acrylic stop was sighted on the centre of the back surface of the cuvette to ensure that only light from this central region was collected. If scattering is insufficient, the diffusion approximation breaks down. Light scattered through these 'sub-diffusive' paths will have a residual polarisation of that of the incident beam whereas diffuse light is unpolarised. The polariser, which is crossed with the incident beam, is used to exclude these sub-diffusive paths. The output of the PMT was autocorrelated at a Flex02OEM-12C programmable hardware correlator (correlator.com). The correlator was provided as a PCB and was supplied with c source code and a .dll library - some work was required to render it operational (rather mysteriously it only worked with some host PCs). The correlator timing was verified using a precision optical chopper. The correlation resulting from modulating the intensity with a chopper is shown in Fig. 6.2. The correlation shows a triangular waveform at intermediate delay times with a period of the chopping period aliasing is present at longer times.

The optical set-up for the PCS microrheology experiment is shown in Fig. 6.1 ii. This experiment was set up on a Malvern 4700 Autosizer. Light from a polarised 500 $\mathrm{mW}, \lambda=532 \mathrm{~nm}, \mathrm{Nd}$ :Yag laser (JDS uniphase) was focused and directed into a $7 \mathrm{~mm}$ diameter cylindrical cuvette containing the sample mounted in a water bath. The water bath was used to minimise both scattering and focusing by the exterior surface of the cuvette and for temperature control. Scattered light was spatially filtered by a beam stop used to localise the scattering volume, and collected at an avalanche photo diode (APD) supplied with the Autosizer. Correlation was performed on the Flex02OEM-12C. The spatial filter/APD were goniometer mounted. A scattering angle of $\theta=20 \mathrm{deg}$ was chosen to minimise the contribution of scattering from the wormlike micelles to the correlation 

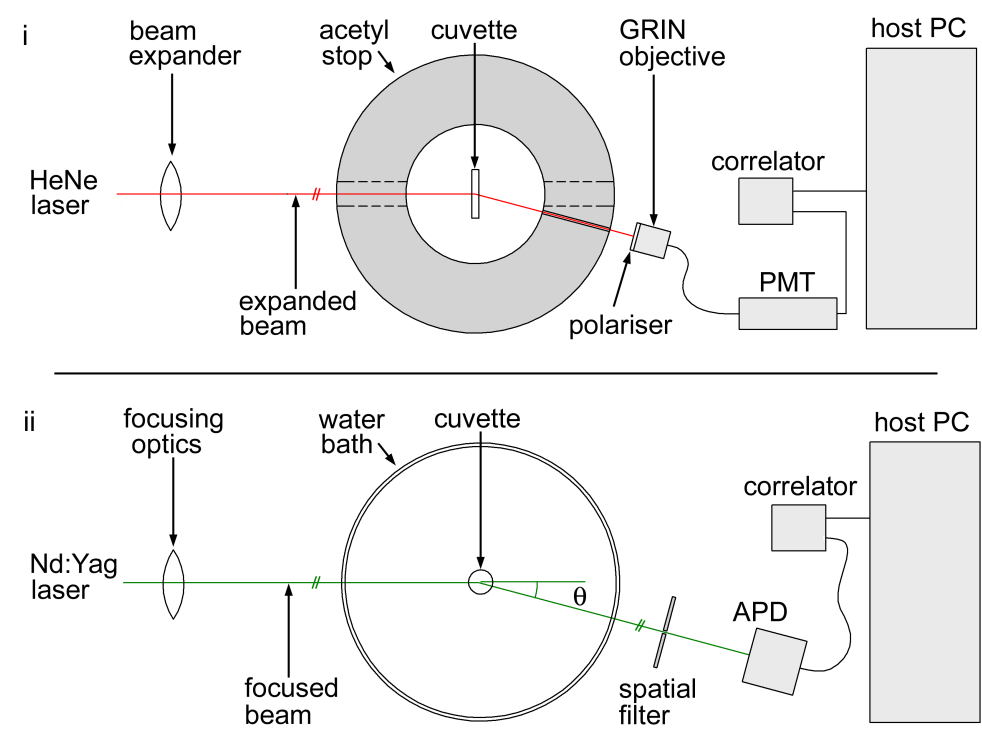

Figure 6.1: Experimental set-up for the i) DWS and ii) PCS microrheology measurements.
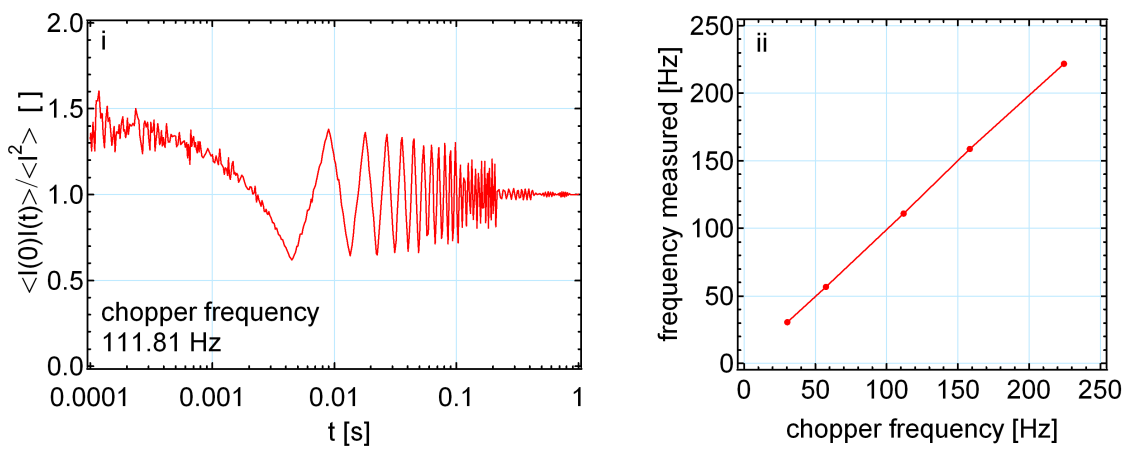

Figure 6.2: Confirming timing of the correlator with a precision optical chopper. 
while keeping the total measurement time practical.

In both PCS and DWS the measured intensity correlation is generally of the form

$$
\frac{\langle I(0) I(t)\rangle}{\langle I\rangle^{2}}=1+\beta\left|g_{1}(t)\right|^{2} .
$$

$\beta$ is a coherence factor that depends on characteristic speckle size, collection area and depolarisation effects (Fig. 4.7 demonstrates that depolarisation affects $\beta$ ). If the collection area is smaller than the characteristic speckle size and depolarisation effects can be neglected $\beta \approx 1$. $\beta$ can be determined experimentally by extrapolating the correlation back to a delay time $t=0$. In all cases here $\beta \approx 1$. The measured DWS and PCS correlation $\left|g_{1}(t)\right|^{2}$ are shown in Fig. 6.3 i-ii. The peak emerging at delay time $t \approx<1 \times 10^{-6}$ is a PMT artefact called after-pulsing. This feature can make the determination of the coherence factor difficult if the characteristic times of the correlation are small. This was not a problem here however. It can be eliminated by cross-correlating the output of two matched PMTs as will be demonstrated in chapters 7 and 8 .

The measured $\left|g_{1}(t)\right|^{2}$ correlation in the DWS experiment (Fig. 6.3 i) was numerically inverted assuming values of $l^{*}=0.25 \mathrm{~mm}$ (Fig. 4.4 ii) and $\gamma_{0}=1.7$ [11] using Eqn. 4.79 to give the probe's MSD (Fig. 6.3 iii). From the data, it is seen that $\left\langle\Delta r^{2}(t)\right\rangle \propto t$ between $t \approx 10^{-6}-10^{-5} \mathrm{~s}$ and $t \approx 3-30 \mathrm{~s}$ indicating free diffusion of the probes. On intermediate time scales $\left\langle\Delta r^{2}(t)\right\rangle \propto t^{p}$ with $p<1$, the probe diffusion is sub-diffusive. The measured MSD was smoothed using an interpolating spline and $G^{*}(\omega)$ calculated using Eqn. 6.3, 6.4 and 6.5. The mechanical and optical rheological spectra are compared in Fig. 6.3. The optical spectrum shows many similarities to the mechanical spectrum and qualitatively shows the behaviour predicted by the Poisson-renewal model. However, there is a clear difference in the amplitude $G^{*}(\omega)$ between the optical and mechanical measurements. The amplitude of $G^{*}(\omega)$ is sensitive to the choice of $l^{*}$. Even in cases where $l^{*}$ has been measured independently using static light scattering, the optically determined $G^{*}(\omega)$ has been found to differ from mechanical measurements by factors of up to 1.5 in wormlike micelles solutions [112], suggesting that the mismatch in amplitude observed may not be unexpected.

The measured $\left|g_{1}(t)\right|^{2}$ correlation in the PCS experiment (Fig. 6.3 ii) was numerically inverted assuming values of $q=(4 \pi \times 1.33) /\left(532 \times 10^{-9}\right) \times \sin [20 \pi / 360]=5.46 \times 10^{6}$ $\mathrm{m}^{-1}$ using Eqn. 4.60 to determine the MSD (Fig. $6.3 \mathrm{iv}$ ) and the MSD inverted using Eqn. 6.3, 6.4 and 6.5 (shown in Fig. 6.3 iii). As was indicated by the MSD inferred from the DWS measurements at both short and long times, the probe particles undergo free diffusion. At intermediate times, the probe's diffusion is restricted. In this case, the mismatch between the optical and mechanical $G^{*}(\omega)$ measurements is more dramatic.

The characteristic time of $g_{1}(t)$ for free diffusion in PCS is $\tau_{\mathrm{PCS}}=\left[q^{2} D\right]^{-1}$, whereas in a DWS measurement it is $\tau_{\text {DWS }} \approx\left[2 k^{2} D / n_{p}\right]^{-1}$ where $n_{p}$ is the most probable number of steps in the light path which occurs at the maximum of $G_{n}\left(r_{i}, r_{o}, l^{*}\right)$. In a transmission DWS measurement $n_{p}$ is typically around $n_{p} \approx 10$. For smaller angles $\sin ^{2} \theta \approx 0.01-0.1$ leading to a ratio of characteristic times $\tau_{\mathrm{PCS}} / \tau_{\mathrm{DWS}} \sim 100-1000$. As a consequence, 

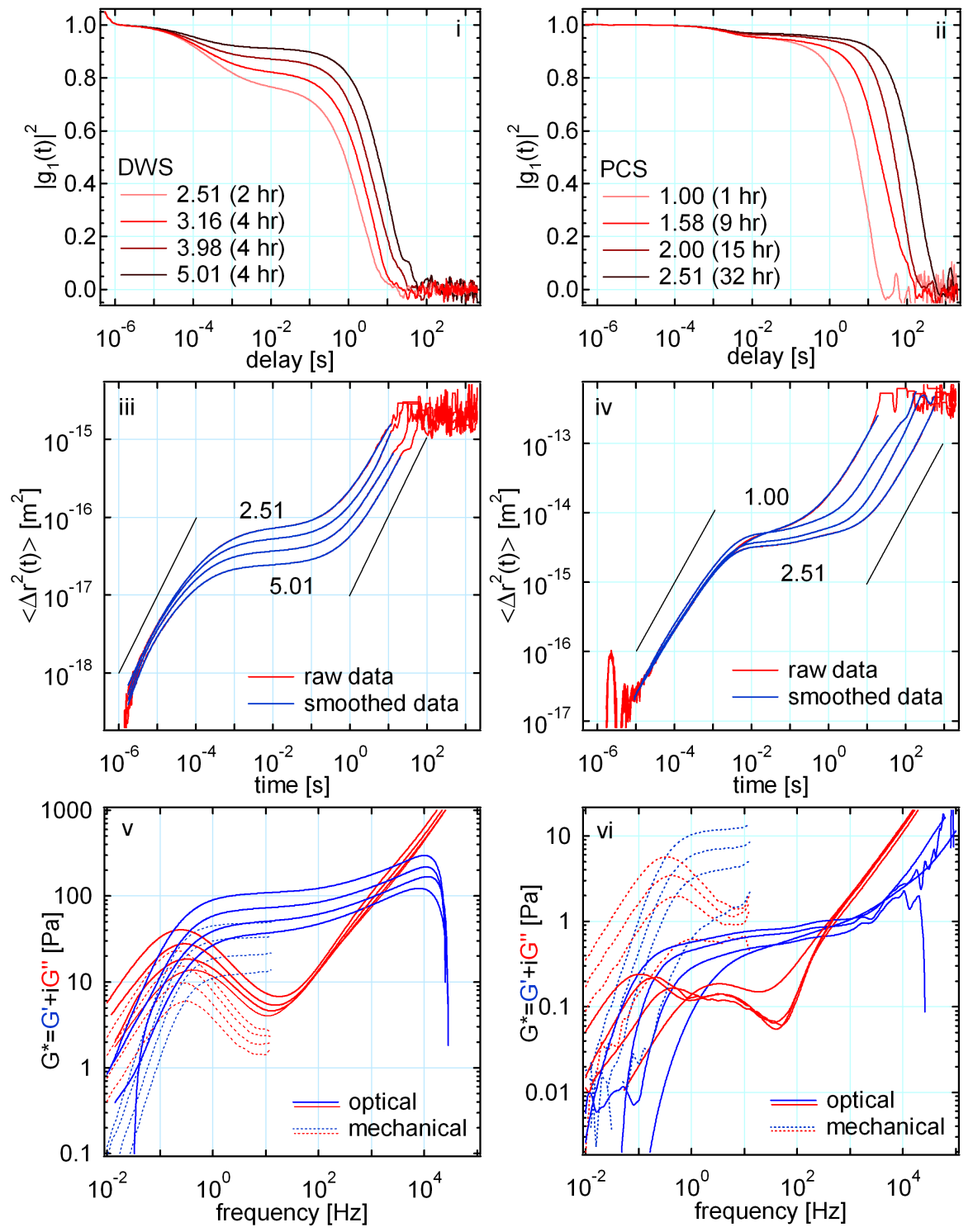

Figure 6.3: DWS and PCS microrheological measurements of the RHB samples at the weight fraction indicated. i-ii) Normalised DWS and PCS correlations were used to determine iii-iv) the MSD of the probes and v-vi) $G^{*}(\omega)$ of the host medium. 
PCS measurements take far longer and are much more likely to be contaminated by the diffusion of impurities (dust) through the beam in the water bath. The undoped wormlike micelles solutions examined showed weak isotropic scattering whereas the larger probes preferentially scatter light in the forward direction. The time dependence of these two scattering processes will almost certainly be different - scattering from the micelles effectively contaminates the measurements of $G^{*}(\omega)$. To minimise this contamination, measurements can be made at smaller angles where scattering from the probes dominates. However, this increases the characteristic time $\tau_{\mathrm{PCS}}$ and sensitivity of the measurement to dust. The concentration of probes can be increased but this may lead to multiple scattering. The intensity of the light scattered in PCS is generally much smaller than that observed in DWS. To compensate, a more powerful laser can be used. However, this can lead to beam heating of the sample (the $500 \mathrm{~mW}$ laser used for the PCS measurements was sufficiently powerful to make black tape smoke at the beam waist). The difference between the optical and mechanical spectra may be due to contamination by dust, contamination by scattering from the micelles, or beam heating.

\subsubsection{Mechanical rheometry}

As noted earlier, inertia influences the measured rheology at high frequencies, potentially obscuring the effect of high frequency modes. In the last section the low inertia technique, microrheology was used to reveal these modes. In this section the influence of these modes on the linear rheology is revealed using conventional rheometry with samples carefully selected for their slow dynamics. Here, wormlike micelles solutions composed of: 1) 42.5 $\mathrm{g} / \mathrm{L}$ of phenylethanol (Alfa Aesar) in an aqueous 5 wt.\% P105 pluronic (BASF) solution and 2) an RHA wormlike micelles solution with $[\mathrm{CPCl}]=0.100 \mathrm{M}$ and $[\mathrm{NaSal}]=0.065 \mathrm{M}$ (Sigma-Aldrich) in water were examined as a function of temperature using conventional mechanical rheometry.

P105 pluronic is a three unit block copolymer PEO-PPO-PEO composed of polyethylene oxide and polypropylene oxide. Because of differences in the hydrobicity of the PEO and PPO structural units, it behaves like a 'gemini' surfactant with a hydrophobic PPO head and two hydrophillic PEO tails. In this solution, it forms inverted (or hairy) wormlike micelles with the PPO head at the core and PEO tails directed out into solution (see [113] and references therein).

Measurements of the linear rheology with temperature are shown in Fig. 6.4. The measurements of the P105 sample were challenging because of the system's extremely slow dynamics. Because of the long time (1-2 days) required to resolve the low frequency behaviour, special care was taken to avoid evaporation.

The P105 wormlike micelles solution showed an increase in turbidity on heating - an increase in turbidity on heating was not observed in the other wormlike micelles solutions studied here. This is illustrated in Fig. 6.4 v which shows a series of photographs of the sample in a glass cuvette equilibrated at the temperature indicated for an hour. At around $T=27^{\circ} \mathrm{C}$ the turbidity of the sample begins to sharply increase - this increase 

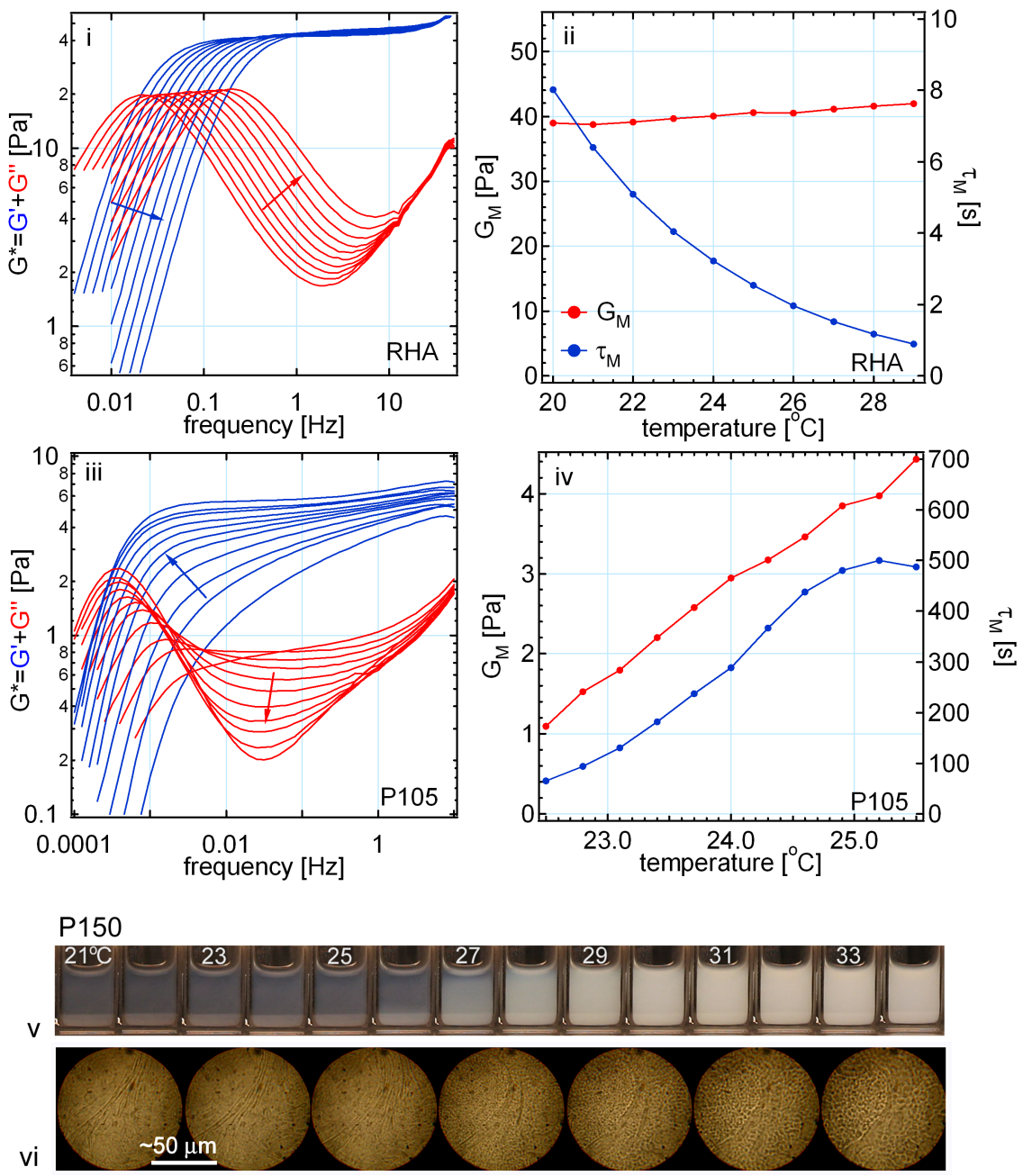

Figure 6.4: Mechanical measurements of the linear rheology of a i-ii) RHA and iii-iv) P105 wormlike micelles solution with temperature. Arrows indicate increasing temperature. v) Direct observation of the P105 wormlike micelles solution sample with increasing temperature. vi) Optical micrographs of the P105 sample on heating. 
in turbidity may signal that a phase transition is occurring. Fig. 6.4 vi shows optical transmission micrographs of the same sample between coverslips with gentle heating from ambient with a hairdryer. A dramatic change in texture is observed on heating, again suggesting a phase transition. Turbidity was not observed when the sample was cooled down to temperatures of $T=4{ }^{\circ} \mathrm{C}$.

\subsubsection{Analysis}

The Poisson-renewal model depends on five parameters: 1) $G_{0}$, the initial shear modulus which scales the amplitude of the complex shear modulus $\left.\left(G^{*}(\omega) \propto G_{0}\right) ; 2\right)$ the ratio $l_{e} / \bar{l}$, where $l_{e}$ is the entanglement length and $\bar{l}$ is the average length of the wormlike micelles; 3 ) the ratio $\tau_{D 0} / \tau_{b}$, where $\tau_{D 0}$ is a scaled reptation time $\left(\tau_{D 0}=\tau_{r e p} / \pi^{2}\right)$ and $\tau_{b}$ is the characteristic breakage and recombination time; 4) $\alpha$, which relates the amplitude of the initial shear modulus $G_{0}$ to the elastic shear modulus $G_{e}=G_{0}\left(1-\alpha \times l_{e} / \bar{l}\right)-$ in [15] a constant value of $\alpha=1$ was assumed and 5) $\omega / \tau_{D 0}$ which is scaled angular frequency. Typical behaviour predicted by the Poisson-renewal model is shown in Fig. 6.5 ii-iii. The parameters chosen for the curves are listed in Table 6.1.

$G_{0}$ and $\tau_{D 0}$ are scaling factors which scale the amplitude and frequency offsetting $\log G^{*}(\omega)$ vertically or horizontally. The dependence on the remaining parameters $l_{e} / \bar{l}$, $\tau_{D 0} / \tau_{b}$ and $\alpha$ is illustrated in Fig. 6.5 iii-iv as a function of $\omega / \tau_{D 0}$. As the ratio $\tau_{D 0} / \tau_{b}$ increases, the local maximum in $G^{\prime}$ (at $\left[\omega_{L O}, G_{L O}\right]$ - see Fig. 6.5 i) is shifted to higher frequencies producing a more Maxwell-like response. As the ratio $l_{e} / \bar{l}$ is reduced, the high frequency intercept (at $\left.\left[\omega_{H I}, G_{L O}\right]\right)$ along with the local minimum in $G^{\prime \prime}$ (at $\left[\omega_{\text {min }}, G_{\text {min }}^{\prime \prime}\right]$ ) is shifted to higher frequencies while the depth of the $G^{\prime \prime}$ minimum increases $\left(G_{\min }^{\prime \prime}\right.$ decreases). Increasing $\alpha$ reduces the depth of the $G^{\prime \prime}$ minimum $\left(G_{\min }^{\prime \prime}\right.$ increases) having little effect on the high frequency intercept $\left(\left[\omega_{H I}, G_{L O}\right]\right)$.

Granek and Cates [15] noted that the Poisson-renewal model predicted that

$$
\frac{G_{\min }^{\prime \prime}}{G_{e x t}^{\prime}} \geq \frac{l_{e}}{\bar{l}}
$$

where $G_{\text {ext }}^{\prime}$ is an estimate of $G_{e}$ obtained by extrapolation of a tangent to the parametric $G^{\prime}(\omega)$ versus $G^{\prime \prime}(\omega)$ Cole-Cole plot to a point $G^{\prime \prime}(\omega)=0$. The quantity $2 G_{L O} \approx G_{e}$ (roughly) and requires no extrapolation. The dependence of the ratio $G_{m i n}^{\prime \prime} /\left(2 G_{L O}\right)$ on both $l_{e} / \bar{l}$ and $\tau_{D 0} / \tau_{b}$ is illustrated in Fig. $6.6 \mathrm{i}$ for $\alpha=1$. For smaller values of $\tau_{D 0} / \tau_{b}$ the ratio $G_{\min }^{\prime \prime} /\left(2 G_{L O}\right)$ is indeed close to the ratio $l_{e} / \bar{l}$. For larger values of $\tau_{D 0} / \tau_{b}$ the ratio is larger than $l_{e} / \bar{l}$. It is noted here that the ratio $\omega_{\min } / \omega_{L O}$ also depends on $l_{e} / \bar{l}$ and $\tau_{D 0} / \tau_{b}$ - this dependence is illustrated in Fig. 6.6 ii. Fig. 6.6 i and 6.6 ii can be combined to give the graphical solution shown in Fig. 6.6 iv. This graphical solution can be used to determine $l_{e} / \bar{l}$ and $\tau_{D 0} / \tau_{b}$ from measurements of $G_{\min }^{\prime \prime} /\left(2 G_{L O}\right)$ and $\omega_{\min } / \omega_{L O}$ (for $\alpha=1$ ). The dependence of the ratio $\kappa=\tau_{L O} / \tau_{D 0}$, where $\tau_{L O}=1 / \omega_{L O}$ on $l_{e} / \bar{l}$ and $\tau_{D 0} / \tau_{b}$, is illustrated in Fig. 6.6 iii. Clearly, $\kappa$ shows a strong dependence on $\tau_{D 0} / \tau_{b}$ but only weak dependence on $l_{e} / l$. These data can be used to construct the second graphical 

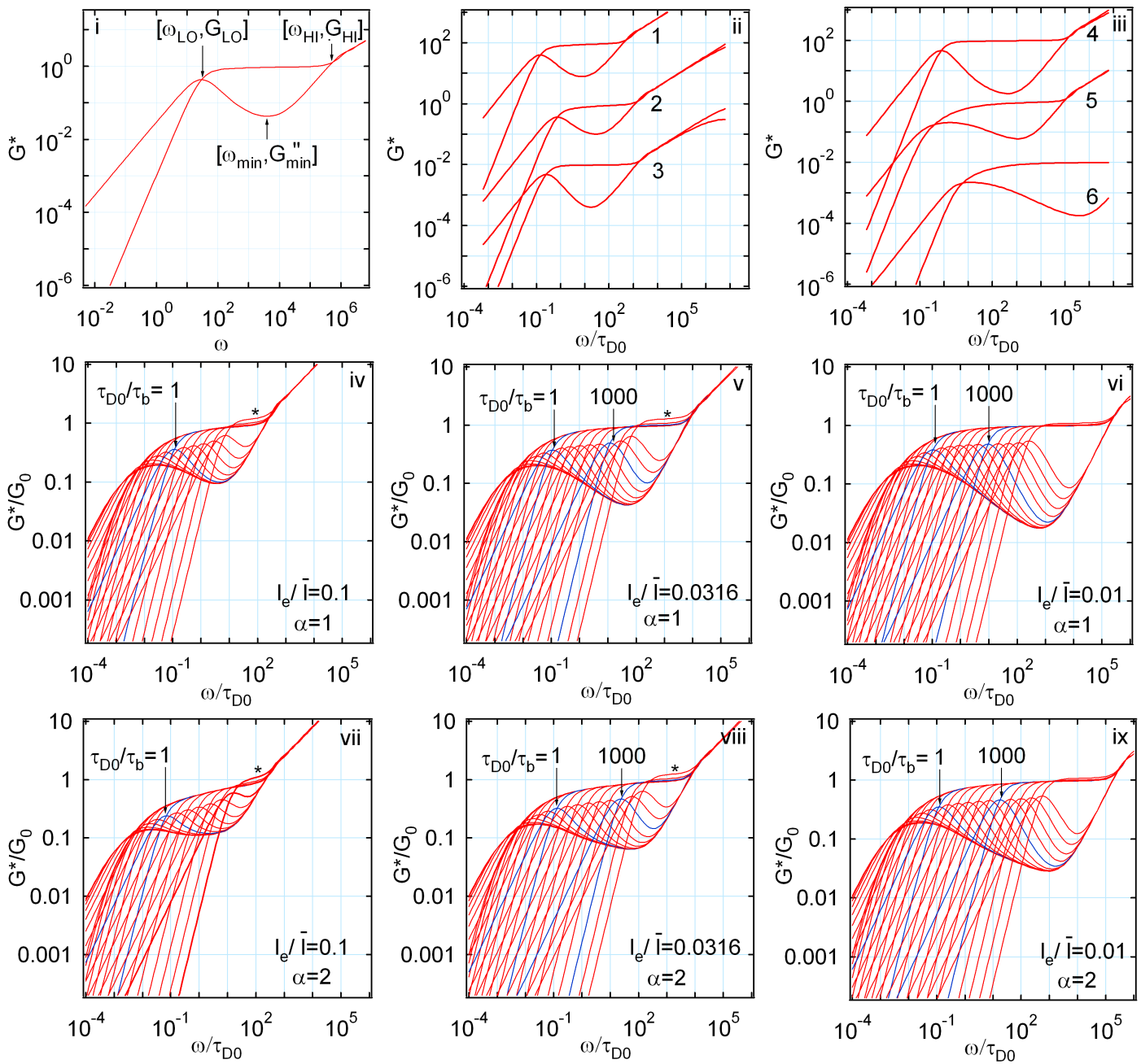

Figure 6.5: Dependence of the Poisson-renewal model on adjustable parameters. i) Model $G^{*}(\omega)$ data with points of interest. ii-iii) Model $G^{*}(\omega)$ for parameters listed in table 6.1. Dependence of $G^{*}(\omega)$ on the parameters $\tau_{D 0} / \tau_{b}, l_{e} / \bar{l}$ and for iv-vi) $\alpha=1$ or for vi-ix) $\alpha=2$. 

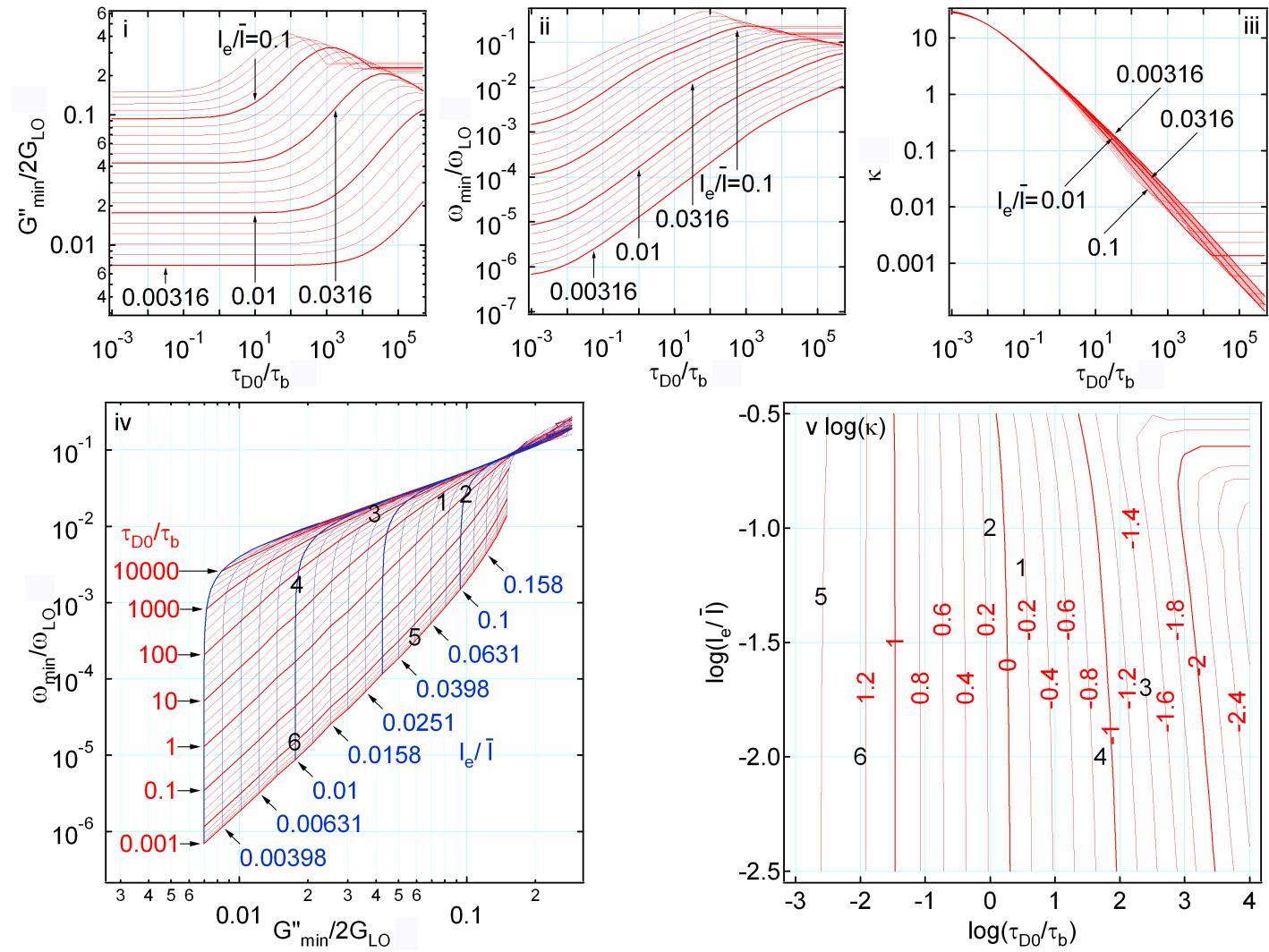

Figure 6.6: i) Dependence on $l_{e} / \bar{l}$ and $\tau_{D 0} / \tau_{b}$ of i) $G_{m i n}^{\prime \prime} /\left(2 G_{L O}\right)$, ii) $\omega_{L O} / \omega_{\min }$ and iii) $\kappa=\tau_{L O} / \tau_{D 0}$. i-ii can be used to construct the graphical solution iv) which can be used to determine $\tau_{D 0} / \tau_{b}$ and $l_{e} / \bar{l}$ from measurements of $\omega_{L O} / \omega_{\min }$ and $G_{\min }^{\prime \prime} /\left(2 G_{L O}\right)$. iii can be used to construct the graphical solution v) which can be used to determine the ratio $\kappa=\tau_{L O} / \tau_{D 0}$ from $\tau_{D 0} / \tau_{b}$ and $l_{e} / \bar{l}$.

solution shown in Fig. 6.6 v. Using values of $l_{e} / \bar{l}$ and $\tau_{D 0} / \tau_{b}$ from the first graphical solution, $\kappa$ and $\tau_{D 0}=\kappa \tau_{L O}$ can be determined. $G_{0}$ can be determined using the relation $G_{0}=G_{e x t} /\left(1-l_{e} / \bar{l}\right)$. The graphical solution is demonstrated using data shown in Fig. 6.5 ii-iii. $G_{e}$ is compared to $G_{e x t}$ and $2 G_{L O}$ in table 6.2. As illustrated in this table, $G_{e x t}$ is generally a much better estimate of $G_{e}$ than $2 G_{L O}$ and should be used to estimate $G_{e}$ and $G_{0}=G_{e} /\left(1-l_{e} / \bar{l}\right)$.

The preceding analysis presupposes that the Poisson-renewal model is an accurate description of the linear rheology of wormlike micelles solutions. To demonstrate that the model is accurate, it must be demonstrated that the model can reproduce experimental data (within experimental error). To verify that the model is an accurate description of the rheology of wormlike micelles, experimental data were fitted to the Poisson-renewal 


\begin{tabular}{|l|l|l|l|l|l|l|l|l|}
\hline No. & $G_{0}$ & $\tau_{D 0}$ & $l_{e} / \bar{l}$ & $\tau_{D 0} / \tau_{b}$ & $\tau_{L O}$ & $\omega_{L O} / \omega_{\min }$ & $G_{\min }^{\prime \prime} /\left(2 G_{L O}\right)$ & $\log \kappa$ \\
1 & 100 & 10 & 0.067 & 3 & 7 & 0.021 & 0.078 & -0.16 \\
2 & 1 & 1 & 0.10 & 1 & 1.3 & 0.026 & 0.10 & 0.14 \\
3 & 0.01 & 100 & 0.02 & 250 & 4.0 & 0.014 & 0.039 & -1.4 \\
4 & 100 & 10 & 0.01 & 50 & 1.3 & 0.0017 & 0.018 & -0.88 \\
5 & 1 & 0.1 & 0.05 & 0.0025 & 2.6 & 0.00035 & 0.059 & 1.4 \\
6 & 0.01 & 1 & 0.01 & 0.01 & 0.17 & 0.000015 & 0.018 & 1.2 \\
\hline
\end{tabular}

Table 6.1: Parameters used in the calculation of the model data presented in Fig. 6.5 i-ii). $\tau_{L O}, \omega_{L O} / \omega_{\min }$ and $G_{\min }^{\prime \prime} /\left(2 G_{L O}\right)$ were estimated from the data shown in Fig. 6.5. These estimates are plotted in the graphical solution shown in Fig. 6.6 iv-v) and are identified by the numbers 1-6. Note that the locations of the estimates in the graphical solutions are consistent with assumed $l_{e} / \bar{l}, \tau_{D 0} / \tau_{b}$ and $\tau_{D 0}$ values.

\begin{tabular}{|l|l|l|l|l|}
\hline No. & $G_{0}$ & $G_{e}$ & $G_{\text {ext }}$ & $2 G_{L O}$ \\
1 & 100 & 93 & 90 & 79 \\
2 & 1 & 0.90 & 0.90 & 0.72 \\
3 & 0.01 & 0.0095 & 0.0095 & 0.0094 \\
4 & 100 & 99 & 97 & 92 \\
5 & 1 & 0.95 & 1.0 & 0.34 \\
6 & 0.01 & 0.0099 & 0.01 & 0.0040 \\
\hline
\end{tabular}

Table 6.2: Model $G_{e}$ values for the data presented in Fig. 6.5 i-ii) with a comparison to an estimate based on extrapolated $G_{\text {ext }}$ and $2 G_{L O}$. 
model. That is, the function

$$
\chi^{2}=\sum_{i=1}^{N} \frac{\left[\log G_{i, \exp }^{\prime}-\log G_{i, m o d}^{\prime}\right]^{2}}{\sigma_{i}^{\prime 2}}+\frac{\left[\log G_{i, \text { exp }}^{\prime \prime}-\log G_{i, \text { mod }}^{\prime \prime}\right]^{2}}{\sigma_{i}^{\prime \prime 2}},
$$

where $G_{i, \text { exp }}^{\prime}$ and $G_{i, \text { exp }}^{\prime \prime}$ are the experimental data and $G_{i, m o d}^{\prime}\left(G_{0}, \tau_{D 0}, l_{e} / \bar{l}, \tau_{D 0} / \tau_{b}, \alpha\right)$ and $G_{i, \text { mod }}^{\prime \prime}\left(G_{0}, \tau_{D 0}, l_{e} / \bar{l}, \tau_{D 0} / \tau_{b}, \alpha\right)$ are model data points, was optimised to minimise $\chi^{2} . \sigma_{i}^{\prime 2}$ and $\sigma_{i}^{\prime \prime 2}$ are weighting factors describing the experimental variance in the (log scaled) data due to random and systematic errors. These variances were not measured. For higher viscosity samples, the 'scatter' in the $\log G^{*}(\omega)$ measurements appeared to be roughly uniform - it was assumed here that the variance in the log scaled data was constant for all measurements, that is, $\sigma_{i}^{\prime 2}=\sigma_{i}^{\prime \prime 2}=$ constant. If these weighting factors are known, the minimum $\chi^{2}$ value can be interpreted quantitatively. Because these variances were not known, a quantitative interpretation is not possible and the fit will be interpreted qualitatively here.

Because the calculation of $G^{*}(\omega)$ is computationally intensive, a method based on both grid searching and conventional fitting was employed to minimise the total number of $G^{*}(\omega)$ evaluations. Here $G_{\text {grid }}^{*}$ was evaluated on a four dimensional, $l_{e} / \bar{l} \times \tau_{D 0} / \tau_{b} \times \alpha \times \omega$, $40 \times 90 \times 12 \times 51$ grid for $l_{e} / \bar{l}=0.01-0.891, \tau_{D 0} / \tau_{b}=10^{-3}-10^{6}, \alpha=0.25-3.00$, $f=10^{-4}-10^{6} \mathrm{~Hz},\left(l_{e} / \bar{l}, \tau_{D 0} / \tau_{B}\right.$ and $f$ were log spaced, $\alpha$ was linearly spaced in 0.25 increments) for $G_{0}=1$ and $\tau_{D 0}=1$. $G_{\text {grid }}^{*}$ took about a month to evaluate on a $2 \mathrm{GHz}$ $\mathrm{PC}$. The experimental data were fitted using a conventional least squares fitting technique (Levenberg-Marquart) at each of $40 \times 90 \times 12 l_{e} / \bar{l} \times \tau_{D 0} / \tau_{b} \times \alpha$ values to determine $40 \times 90 \times 12$ best fitting $G_{0}$ and $\tau_{D 0}$ values along with $40 \times 90 \times 12 \chi^{2}$ values. The overall best fitting combination $G_{0}, \tau_{D 0}, l_{e} / \bar{l}, \tau_{D 0} / \tau_{b}$ and $\alpha$ corresponds to the lowest $\chi^{2}$ value. The fit procedure is illustrated in Fig. 6.7. Here $G_{\text {grid }}^{*}$, calculated for particular $l_{e} / \bar{l}, \tau_{D 0} / \tau_{b}$ and $\alpha$, is scaled by test values of $G_{0}$ and $\tau_{D 0}$ to give $G_{\text {mod }}^{*} . G_{0}$ and $\tau_{D 0}$ are optimised to minimise the difference between the model and experimental data $G_{\text {exp }}^{*}$ (Fig. 6.7 i). The minimum $\chi^{2}$ value associated with the best fitting $G_{0}$ and $\tau_{D 0}$ at each $l_{e} / \bar{l}, \tau_{D 0} / \tau_{b}$ and $\alpha$ is used to construct three dimensional $\chi^{2}$ surface in $l_{e} / \bar{l}, \tau_{D 0} / \tau_{b}$ and $\alpha$. A slice through this surface is shown for $\alpha=1$ in Fig. 6.7 ii. Note that the surface has a well defined minimum. For $\left(l_{e} / \bar{l}\right)^{3}>0.1 \times \tau_{D 0} / \tau_{b}$ the apparent elastic modulus increases above one. This behaviour is evident in Fig. 6.5 iv-ix for the curves marked with a star $\star$. Values of $\left(l_{e} / \bar{l}\right)$ and $\tau_{D 0} / \tau_{b}$ such that $\left(l_{e} / \bar{l}\right)^{3}>0.1 \times \tau_{D 0} / \tau_{b}$ were excluded from the fit. The excluded region is shown in white in Fig. 6.7 ii.

Seven sets of experimental data were fitted to the Poisson-renewal model. The data, over the frequency domain fitted, along with the best fit are shown in Fig. 6.8. The 'RHBDWS', 'P105-mechanical' and 'RHA-mechanical' measurements were described earlier in this chapter. The 'RHB-mechanical' 'RHC-mechanical' measurements were described in Chapter 5. The 'RHA-literature' data are for a $[\mathrm{CPCl}]=100 \mathrm{mM}$ and $[\mathrm{NaSal}]=60 \mathrm{mM}$ RHA system measured at $T=20-40{ }^{\circ} \mathrm{C}$ using conventional mechanical rheology, DWS microrheology and a recently developed high frequency mechanical rheological technique. These data were extracted from [106]. 

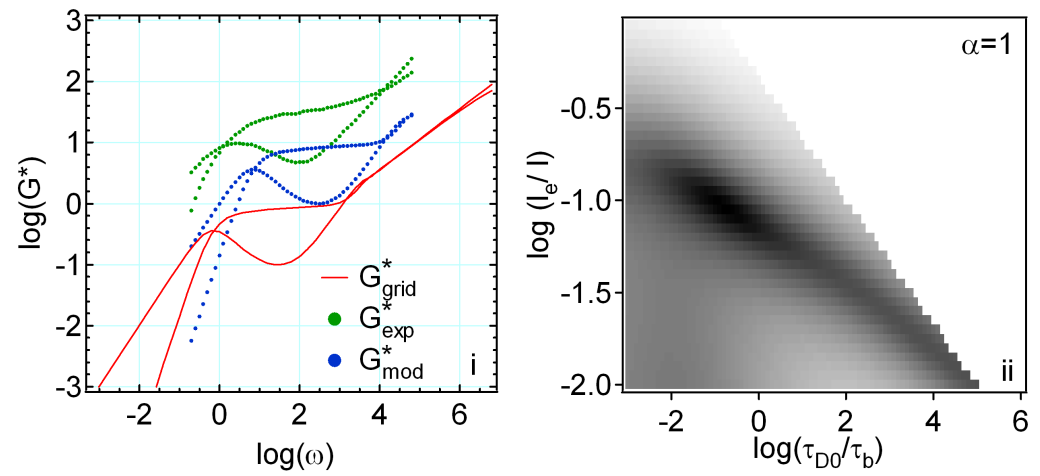

Figure 6.7: i) Fitting the experimental data for particular $l_{e} / \bar{l}, \tau_{D 0} / \tau_{b}$ values. ii) $\log \chi^{2}$ surface as a function of $l_{e} / \bar{l}$ and $\tau_{D 0} / \tau_{b}$ for $\alpha=1$ (darker greys correspond to smaller $\chi^{2}$ values).

The agreement between both the 'RHB-DWS' measurements (Fig. 6.8 i) and the 'P105-mechanical' measurements (Fig. 6.8 iii) and fitted curves is 'poor'. Possible explanations for these differences are discussed later. The agreement between the 'RHAliterature' (Fig. 6.8 ii), 'RHA-mechanical' (Fig. 6.8 iv) and 'RHB-mechanical' (Fig. 6.8 $\mathrm{v}$ and vi) and fitted curves is better. Fig. 6.8 viii-ix compares $l_{e} / \bar{l}, \tau_{D 0} / \tau_{b}, \tau_{D 0}$ and $G_{0}$ determined using the graphical solution with values determined from the fit with $\alpha=1$ for the 'RHA-mechanical' and 'RHB-mechanical' measurements. The graphical solution values and fitted values show reasonable agreement indicating that the two methods are self-consistent.

Fitted parameters are shown in Fig. 6.9 assuming a constant value of $\alpha=1$ in Fig. 6.9 i-vi and allowing the $\alpha$ value to vary in Fig. 6.9 vii-xii. To probe the sensitivity of the fit to the fitted parameters and to get an estimate of the errors, $2 \%$ Gaussian noise was injected into the measured data and this synthetic data set was fitted using the same fitting algorithm six times to give six separate estimates of the fitted parameters (this approach to error estimation is discussed in [114]). Error bars in Fig. 6.9 indicate the standard deviation of each fit parameter over the six fits. Not surprisingly, the inclusion of $\alpha$ as a fitted parameter generally increases the error in the remaining fitted parameters, eliminating the systematic variation seen Fig. 6.9 ii and vi. The ratio $\tau_{D 0} / \tau_{b}$ was calculated from the $\tau_{b} / \tau_{r e p}$ values shown in Fig. 5.3 vi and are included in Fig. 6.9 vi (indicated by square symbols) to demonstrate consistency.

The fitted parameters $l_{e} / \bar{l}, \alpha$ and $G_{e}=G_{0}\left(1-\alpha l_{e} / \bar{l}\right)$ were used to estimate the average length $\bar{l}$ of the wormlike micelles using Eqn. 3.4 and 3.5 . The average length was estimated assuming persistence lengths $l_{p}$ of either 10, 15 or $20 \mathrm{~nm}$ (Granek and Cates assume that $l_{p}=15 \mathrm{~nm}$ in [15]) and all three estimates are shown for $\alpha=1$ and for $\alpha$ unconstrained. Because $\bar{l} \simeq\left(\bar{l} / l_{e}\right) \times\left(\xi^{5 / 3} / l_{p}^{2 / 3}\right)$ increasing $l_{p}$ for a given fitted $\bar{l} / l_{e}$ reduces the estimate of the average length $\bar{l}$. Estimates of the average micelle length are shown 

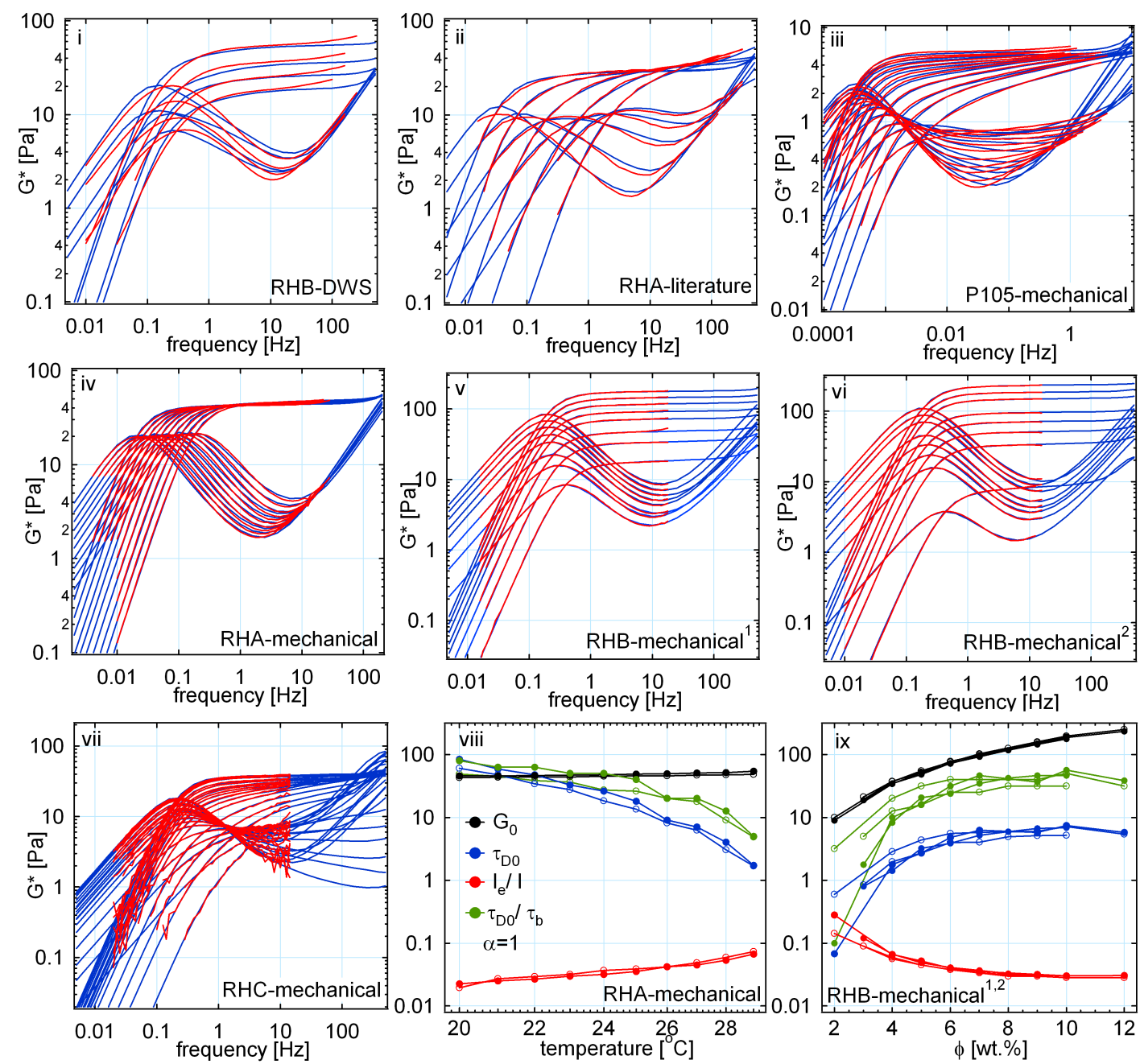

Figure 6.8: i-vii) Experimental data (red) with a five parameter fit (blue) to the Poissonrenewal model. viii-ix) A comparison between parameters determined using the graphical solution (closed circles) and fitted parameters (open circles) for $\alpha=1$ for two systems. ${ }^{1}$ ARES 2.3deg $50 \mathrm{~mm}$ cone-plate. ${ }^{2}$ AR2000 2 deg $40 \mathrm{~mm}$ cone-plate. 

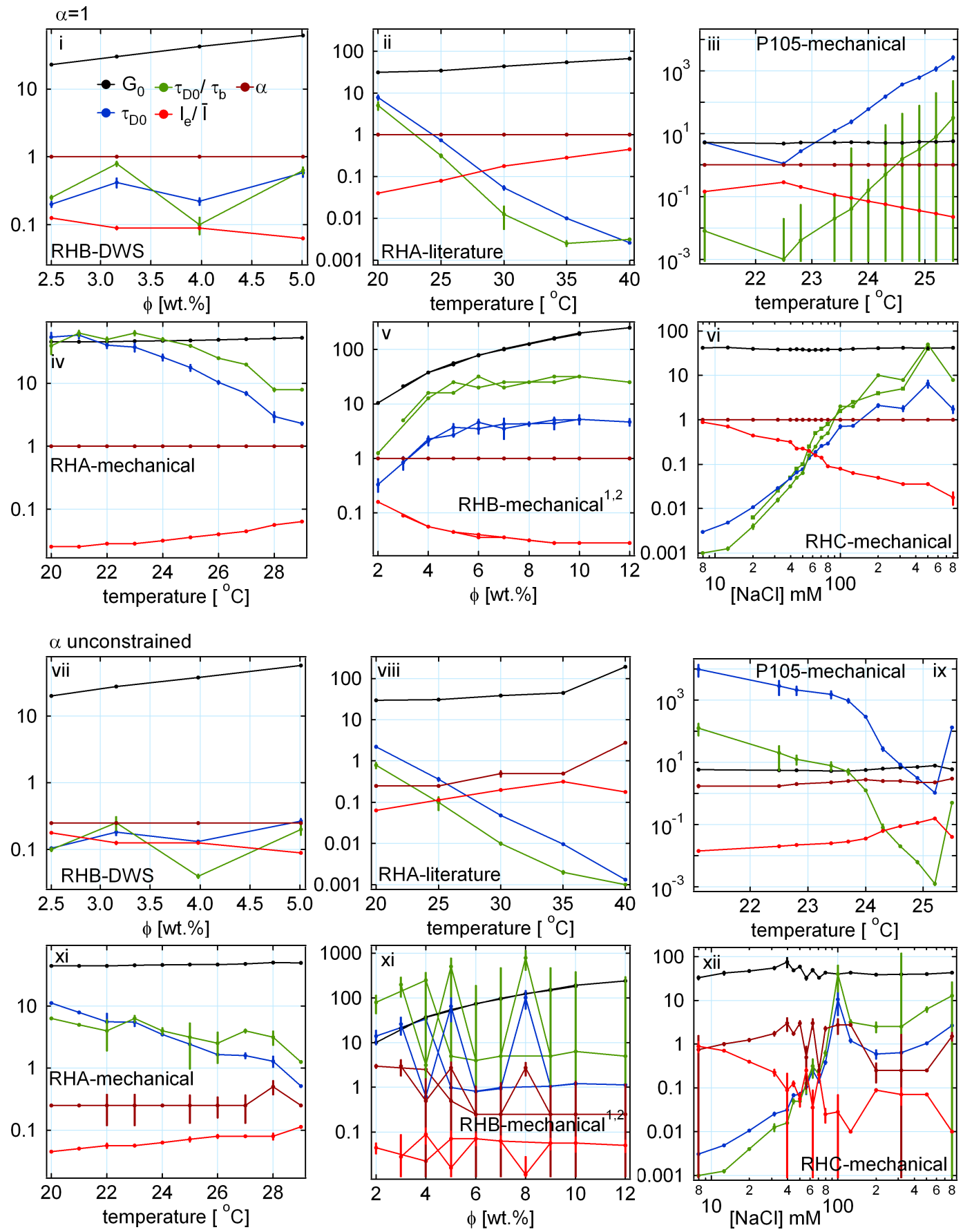

Figure 6.9: Fitted parameters for a i-vi) four $(\alpha=1)$ and vii-xii) five ( $\alpha$ unconstrained) parameter fit to the Poisson-renewal model. 

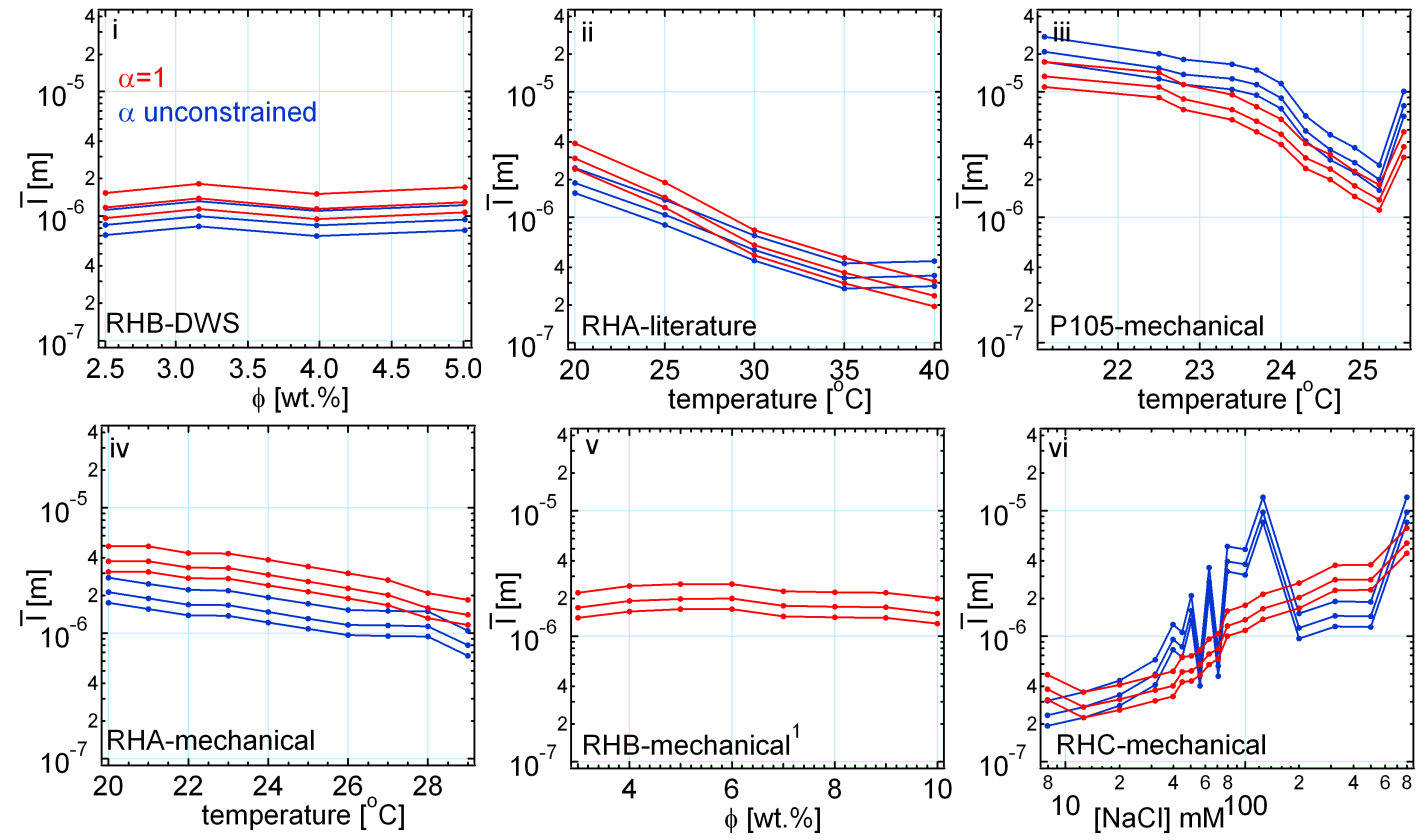

Figure 6.10: Estimate of the average length $\bar{l}$ of the wormlike micelles studied for assumed persistence length $l_{p}=10,15$ and $20 \mathrm{~nm}$.

in Fig. 6.10, The average length of $\mathrm{CPCl}$ wormlike micelles are in the range 0.2 to 4 $\mu \mathrm{m}$. This is consistent with $\bar{l} \simeq 0.61-3.1 \mu \mathrm{m}$ values given for selected CTAC $|\mathrm{NaSal}| \mathrm{NaCl}$ wormlike micelles in [15]. In [15], Granek and Cates noted that "...an anomalous trend of decreasing micelle size with surfactant concentration under conditions of high added salt" in $\mathrm{CTAC}|\mathrm{NaSal}| \mathrm{NaCl}$ system studied. As illustrated by Fig. 6.10 v the average length of the RHB wormlike micelles appears to be roughly constant with increasing surfactant concentration. As shown in Fig. 6.9, the ratio $l_{e} / \bar{l}$ decreases with increasing concentration indicating increased entanglement. This might suggest that increasing the surfactant concentration simply increases the concentration of wormlike micelles in solution. If this were the case, it would be expected that $G_{e} \approx G_{M} \propto \phi_{V}^{2.3}$ and $\eta_{0} \propto \phi_{V}^{3.9-4.7}$ as discussed in chapter 2. This dependence was not observed in chapter 4 however.

\subsection{Diffusion NMR microrheology}

Diffusion NMR is a nuclear magnetic resonance technique which is sensitive to the relative displacement of probe nucleii. It is often used to measure the translational diffusion of hydrogen nucleii in liquids. Because diffusion can be influenced by restrictions, structure can be inferred from these measurements. If the probe molecules were trapped or contained within a much larger structure, for example in the interior of a core-shell particle, it may 


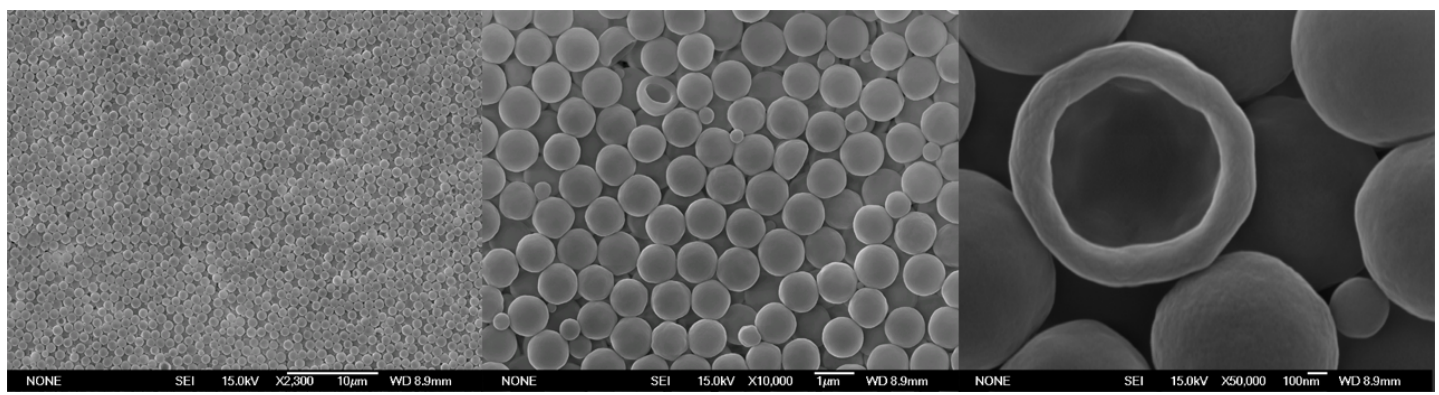

Figure 6.11: Author's SEM micrographs showing commercially available (Polyscience Inc.) core-shell particles at three magnifications. Note that these core-shell particles are monodisperse and have an inner radius $r_{i} \approx 400 \mathrm{~nm}$ and an outer radius $r_{o} \approx 500 \mathrm{~nm}$. The core-shell particles are described as semi-porous.

be possible to infer the diffusion of this larger structure from measurements of the diffusion of the trapped probe molecules. If spherical core-shell particles (like the commercially available core-shell particles shown in Fig. 6.11) are used with an outer diameter much greater than the mesh size, the bulk linear rheology could be inferred from the measured MSD using the technique outlined in previous sections. Because NMR is not an optical technique, the intrinsic turbidity of the host medium is unimportant. As discussed below, diffusion NMR is only sensitive to the Brownian motion along the axis on which the magnetic gradient is applied. Consequently, the technique could potentially be used to probe anisotropy in the linear rheology. The feasibility of a diffusion NMR based microrheology is considered in this section.

In the narrow pulse approximation, the normalised attenuation of the diffusion NMR echo signal $E$ is given by [115]

$$
E(\mathbf{q}, \Delta)=\langle\exp [i 2 \pi \mathbf{q} \cdot \mathbf{r}(\Delta)]\rangle
$$

$\mathbf{r}$ is the displacement of a probe nucleus (often the nucleus of the hydrogen atom) in time $\Delta$ and depends on the specifics of the diffusion process. $\mathbf{q}=\frac{1}{2 \pi} \gamma \delta \mathbf{g}$ is a 'scattering vector' and is dependent on a magnetic gradient pulse direction and strength $\mathbf{g}(\sim 10 \mathrm{~T} / \mathrm{m})$, the magnetic gradient pulse time $\delta(\sim \mathrm{ms})$ and the gyromagnetic ratio of the nucleus $\left(\cong 2.675 \times 10^{-8} \mathrm{rad} \mathrm{s}^{-1} \mathrm{~T}^{-1}\right.$ for hydrogen nucleus). In the narrow pulse approximation, it is assumed that $\delta<<\Delta$. On the NMR diffusion probe, the direction of the magnetic gradient is fixed and only one component of the motion can be observed

$$
E(\mathbf{q}, \Delta)=\langle\exp [i 2 \pi q z(\Delta)]\rangle
$$

If $z(\Delta)$ is a Gaussian variable with zero mean by the central limit theorem

$$
E(\mathbf{q}, \Delta)=\exp \left[-2 \pi^{2} q^{2}\left\langle z^{2}(\Delta)\right\rangle\right]
$$


where $\left\langle z^{2}(\Delta)\right\rangle$ is the $z$ component of the mean square displacement. Eqn. 6.11 describes a Gaussian function. The $z$ component of the $\operatorname{MSD}\left\langle z^{2}(t)\right\rangle=\left\langle z^{2}(\Delta)\right\rangle$ can be determined from $E(\mathbf{q}, \Delta)$ by differentiation $\left.\frac{d E}{d q^{2}}\right|_{q=0}=-2 \pi^{2}\left\langle z^{2}(\Delta)\right\rangle$ or by fitting the data.

The effects of a core-shell restriction on the diffusion of a probe nucleus with diffusion constant $D_{p}$, and on $E(\mathbf{q}, \Delta)$ are demonstrated using simulation in Fig. 6.12. Note that the diffusion constant of water $D_{p}=2 \times 10^{-9} \mathrm{~m}^{2} / \mathrm{s}$. Fig. 6.12 i shows the $E(\mathbf{q}, \Delta)$ when diffusion of the probe nucleii is unrestricted for time $\Delta=1 \times 10^{-6}$ to $10 \mathrm{~s}$ for log space $q$. The corresponding $\left\langle z^{2}(\Delta)\right\rangle$ shown in 6.12 iii can be evaluated by fitting the $E(\mathbf{q}, \Delta)$ to a Gaussian function or directly from the simulated positions of the probe nucleus $-\left\langle z^{2}(\Delta)\right\rangle=$ $2 D_{p} t$ as expected. The effects of a core-shell restriction are illustrated in Fig. 6.12 ii. Here the probe nucleus with $D_{p}=2 \times 10^{-9} \mathrm{~m}^{2} / \mathrm{s}$ is trapped in a cavity of radius $r_{i}=400$ $\mathrm{nm}$. The cavity itself is assumed to diffuse with a diffusion constant $D_{s}=4.4 \times 10^{-13}$ $\mathrm{m}^{2} / \mathrm{s}$. This is the diffusion constant of a sphere of radius $r_{o}=500 \mathrm{~nm}$ in water calculated according to the Stokes-Einstein relation ( $\mathrm{T}=300 \mathrm{~K}, \eta=0.001$ Pa.s) - the water outside the cavity is ignored in this particular simulation. The $\left\langle z^{2}(\Delta)\right\rangle$ corresponding to $E(\mathbf{q}, \Delta)$ is illustrated in Fig. 6.12 iv. For times $\Delta<10^{-5} \mathrm{~s}\left\langle z^{2}(\Delta)\right\rangle=2 D_{p} \Delta$. Here diffusion of the probe is largely unaffected by the restriction. For $10^{-4}<\Delta<10^{-2} \mathrm{~s}\left\langle z^{2}(\Delta)\right\rangle$ plateaus at a value $\left\langle z^{2}(\Delta)\right\rangle=0.39 r_{i}^{2}$. This dependency of the plateau MSD on radius is quite comparable to $\left\langle z^{2}(\Delta)\right\rangle=\frac{2}{5} r_{i}^{2}$ calculated analytically in [115]. For $\Delta>10^{-2} \mathrm{~s}$ $\left\langle z^{2}(\Delta)\right\rangle$ begins to increase according to $\left\langle z^{2}(\Delta)\right\rangle=2 D_{s} \Delta+0.39 r_{i}^{2}$. If the dimensions of the cavity are known, the mean square displacement of the sphere in the host medium can be deduced by subtracting the contribution of the restricted diffusion of the probe nucleii from the total measured $\left\langle z^{2}(\Delta)\right\rangle$. The core-shell particles shown in Fig. 6.11 are described as being semi-porous. It could be expected that the concentration of a small probe molecule would come to equilibrium with the concentration outside the cavity (it is assumed here that this exchange process is very slow and can neglected during an NMR measurement). Consequently, probes outside the cavities will also contribute to the measured $E(\mathbf{q}, \Delta)$. Assuming that the spheres themselves do not influence the free diffusion of the probes, $E(\mathbf{q}, \Delta)$ is the sum of the contribution of the probes outside the cavity $E_{F}$ and inside the cavity $E_{R}$ weighted by the volume fraction: $E(\mathbf{q}, \Delta)=(1-\phi) E_{F}(\mathbf{q}, \Delta)+\phi E_{R}(\mathbf{q}, \Delta)$ where $\phi$ is the volume fraction trapped in the interior of the core-shell particle. The resulting $E(\mathbf{q}, \Delta)$ is the sum of two Gaussian functions as shown in Fig. 6.12 iii. If the characteristic times of the two Gaussian functions are sufficiently different $\left\langle z^{2}(\Delta)\right\rangle$ of the cavity can be determined. Alternatively, an impermeable oil-filled core-shell particle could be used. The gyromagnetic ratio depends on the local nuclear environment - the diffusion of a nucleus in a different local environment can be distinguished on the basis of chemical shift.

The window over which diffusion can be measured in an NMR experiment is shown in black in Fig. 6.12 vii-ix. The lower limit on the MSD is set by the mechanical stability of the system $-\left\langle z^{2}(t)\right\rangle=1 \times 10^{-16} \mathrm{~m}^{2}$ is assumed. The minimum $\Delta_{\min }$ is effectively set by the maximum gradient that can be applied - a value of $\Delta_{\min }=1 \mathrm{~ms}$ is assumed here. The maximum useful $\Delta_{\max }$ is dependent on the NMR $T_{1}$ relaxation of the sample which influences signal strength. $T_{1}$ for water is $T_{1} \approx 1 \mathrm{~s} \mathrm{setting} \Delta_{\max } \approx 1 \mathrm{~s}$. The Poisson-renewal 
model was used to calculate $G^{*}(\omega)$ for $\tau_{D 0}=100 \mathrm{~s}, \tau_{D 0} / \tau_{b}=0.1 l_{e} / \bar{l}=0.01, \alpha=1$ and $G_{0}=1,0.316$ or $0.1 \mathrm{~Pa}$ (the corresponding viscosity of these samples is $\eta_{0}=G_{0} \tau_{M} \approx 5,1.2$ and 0.5 Pa.s). The calculated $G^{*}(\omega)$ was inverted using Eqn. 6.2 for $r_{o}=500 \mathrm{~nm}$ sphere to give $\left\langle z^{2}(t)\right\rangle=\left\langle\Delta r^{2}(t)\right\rangle / 3$. The restricted diffusion of water in an $r_{i}=400 \mathrm{~nm}$ cavity is shown in red - it is assumed that this water can be distinguished from water external to the cavity. Based on the simulations, it is expected that the total $\left\langle z^{2}(t)\right\rangle$ of the water is the sum of the restricted diffusion of the water inside the cavity and the diffusion of the cavity itself. This sum is shown in green. Clearly $\left\langle z^{2}(t)\right\rangle$ within the experimental window is dominated by the restricted diffusion of the probe nucleus. Accurately determining $\left\langle z^{2}(t)\right\rangle$ for the cavity will be difficult. In Fig. 6.12 viii a second smaller core-shell particle with $r_{i}=175 \mathrm{~nm}$ and $r_{o}=275 \mathrm{~nm}$ is considered (also available from Polyscience Inc.). This both increases the restriction of the probe and increases diffusion of the cavity. In this instance it seems possible that the diffusion of the cavity could be determined from a measurement of the total $\left\langle z^{2}(t)\right\rangle$ of the restricted water in the diffusing cavity. If the $\left\langle z^{2}(t)\right\rangle$ of the cavity is known, Eqn. 6.2 can be used to determine $G^{*}(\omega)$. Unfortunately, for $G_{0}=0.1 \mathrm{~Pa}$ and $G_{0}=0.316 \mathrm{~Pa}$, the mesh size or granularity of the entangled network is $\xi=350 \mathrm{~nm}$ and $240 \mathrm{~nm}$ and is comparable to the outer diameter of the sphere.

Sensitivity to the diffusion of the cavity can (in principle) be improved by significantly reducing the diffusion constant $D_{p}$ of the probe. This is illustrated in Fig. 6.12 ix. where a hypothetical oil-filled core-shell particle with a probe diffusion constant $D_{p}=2 \times 10^{-13} \mathrm{~m}^{2} / \mathrm{s}$ is considered. Alternatively, a hypothetical core-shell particle honeycombed with restriction might be useful.

\subsection{Discussion}

As noted in the previous section, the fit to the 'DWS-RHB' data was poor. This may be partly an artefact of the DWS microrheology technique used. Fig. 6.13 illustrates the issue. There is a mismatch between the mechanical and optical measurements at low frequency. As shown in Fig. 6.13 ii extracted from [112, this mismatch has been seen elsewhere. The high frequency mechanical rheometry and DWS microrheology measurements discussed in [106] are quite comparable at higher frequencies suggesting that the mismatch may only be a problem at lower frequencies. It is unclear why this mismatch is present. It may be associated with scattering from the micelles.

While the agreement between the mechanical and fitted curves is 'good' for both the RHB and RHC samples, the fitted parameters do not vary smoothly with concentration when $\alpha$ is unconstrained. It seems physically reasonable to expect that these parameters should vary smoothly suggesting that fit is unable to distinguish between these parameters over the particular frequency domain used. This is consistent with the larger error observed.

The fit to the 'RHA-literature' and 'RHA-mechanical' is 'fair' to 'good' - the fitted parameters do vary smoothly and the errors are smaller. These observations tend to vali- 

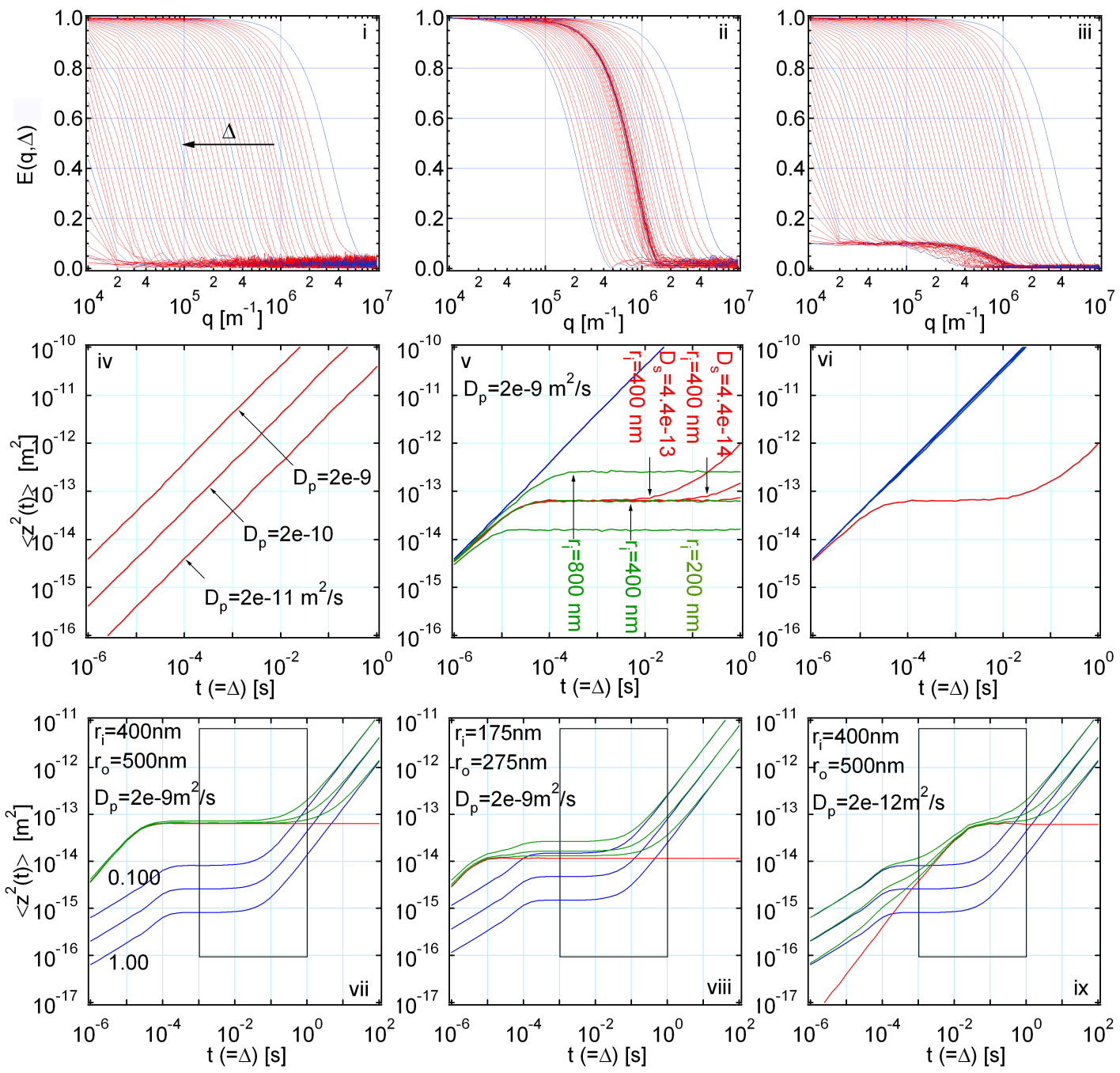

Figure 6.12: Simulated echo attenuation for i) water undergoing free diffusion, ii) water undergoing restricted diffusion in the interior of a core-shell particle and iii) water undergoing both free diffusion outside the core-shell particle and restricted diffusion in the interior of the core-shell particle, with $10 \% \mathrm{~V} / \mathrm{V}$ of the water trapped in the interior of the core-shell particle. iv-vi) MSD derived from the simulated echo attenuation described in i-iii. Calculated MSD of vii) larger and viii) smaller core-shell particles in three wormlike micelles solution matrices with a comparison to the simulated MSD of water trapped in the interior of the (diffusing) core-shell particle, illustrating a possible sensitivity problem with the technique. ix) Simulated MSD of oil trapped in the interior of the (diffusing) core-shell particle. 

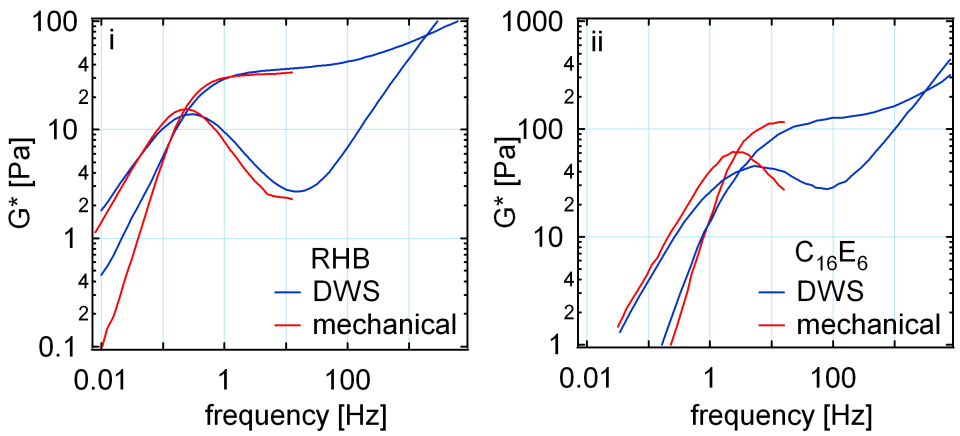

Figure 6.13: Comparison between the mechanical and DWS measurements of the linear rheology of a wormlike micelles solution i) in this chapter and ii) from [106], illustrating a mismatch at low frequencies.

date the Poisson-renewal model. To verify that the Poisson-renewal model is an accurate description of the underlying physical behaviour of wormlike micelles in solution, at least some of the fitted parameters need to be verified as physical using some other method. An estimate of the average length was provided here. While this estimate appears consistent with values given by Granek and Cates in [15, the Granek and Cates estimate was also based on the Poisson-renewal model. As illustrated in Fig. 2.1 micelles can be imaged using SEM (this typically involves 'freeze-fracture' therefore some care may be required as cooling could be expected to influence the micelles length) and the quantities $l_{e}, \bar{l}$ and $l_{p}$ could be determined directly from the micrographs and compared to the values estimated from rheology.

The NMR microrheology technique described here suffers from low sensitivity and a narrow bandwidth and would appear to be difficult to implement with commercially available probes, making the technique of academic interest only. 


\section{Chapter 7}

\section{Studying the flow behaviour of wormlike micelles using diffusing wave spectroscopy}

\subsection{Introduction}

Diffusing wave spectroscopy is sensitive to the relative motion of the scattering centres. As was demonstrated in Chapter 6, the technique's sensitivity to diffusion can be exploited to study the linear rheology of complex fluids. The technique's sensitivity to shear induced relative motion has largely been ignored in rheology. In this chapter, DWS's sensitivity to strain rate is used to investigate the unusual flow behaviour predicted, in Chapter 3, to occur in some wormlike micelles solutions. Here the flow behaviour of four wormlike micelles solutions is studied in the cylindrical-Couette, cone-plate and parallel-plate geometries using DWS. Because of the apparent novelty of the technique discussed here, several other fluids are also studied including a Newtonian fluid (milk), a weakly shear thinning fluid (PEO in water) and a fluid that shows a yield stress (yoghurt) in selected flow geometries.

\subsection{DWS and flow}

In the diffusion approximation, the path of light through a highly multiple scattering medium is treated as a random walk. The scattered field is characterised by a field correlation function which decorrelates with time because of the relative motion of the scattering centres. Fig. 7.1 i illustrates the effects of shear on a typical diffuse path. The red line shows the initial path and the blue line shows the path after the application of shear. This path can be decomposed into a set of path vectors (or bond vectors in the language of polymer physics) $\Delta \mathbf{r}=\mathbf{r}_{n}-\mathbf{r}_{n-1}$, shown in red in Fig. 7.1 ii. The effect of 

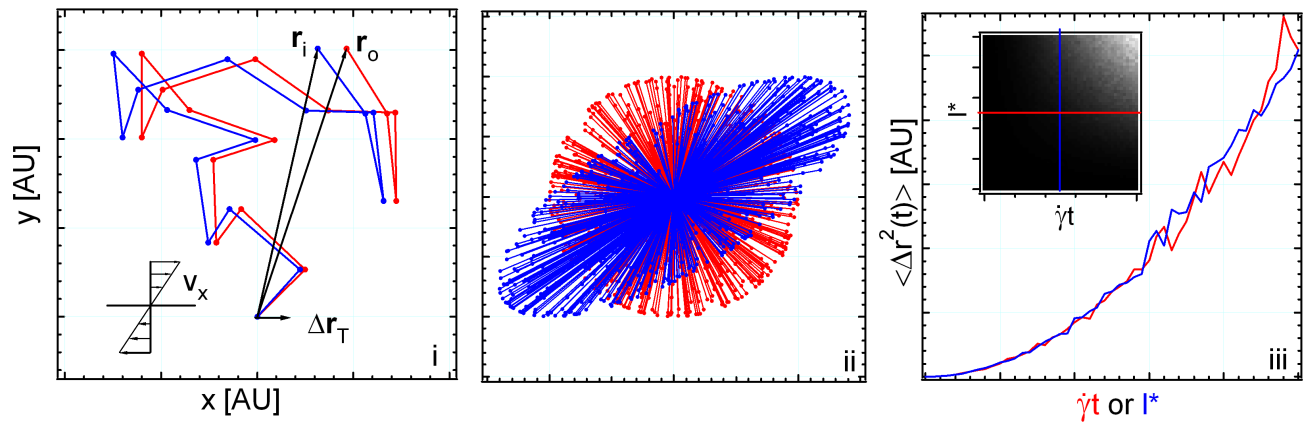

Figure 7.1: i) Shear induced distortion of the light path ii) redistributes the path vectors resulting in iii) a quadratic increase in path lengths with time.

uniform shear on this set of path vectors is shown in blue. In the diffusion approximation, it is expected that the average change in phase of the associated field correlation function depends on the MSD of the scattering centres $\left\langle\Delta \phi_{n}^{2}(t)\right\rangle=2 k^{2}\left\langle\Delta^{2} r(t)\right\rangle$. In Fig. 7.1 iii the MSD of the path vectors as functions of both $\dot{\gamma} t$ and $l^{*}$ is shown. Clearly, the MSD shows quadratic dependence on both parameters, indicating that $\left\langle\Delta r^{2}(t)\right\rangle \propto n \dot{\gamma}^{2} l^{* 2} t^{2}$ which implies that the dephasing of the field correlation function for uniform shear flow is of the form $\left\langle\phi_{n}^{2}(t)\right\rangle \propto n k^{2} \dot{\gamma}^{2} l^{* 2} t^{2}$. Uniform shear flow was examined by Xu et and co-workers in [116]. Here (it is implied that) the dephasing is given by $\left\langle\phi_{n}^{2}(t)\right\rangle=\frac{2}{15} n k^{2} \dot{\gamma}^{2} l^{* 2} t^{2}$ where the prefactor is associated with averaging the $\sin ^{2} \theta \cos ^{2} \phi$ over a unit sphere. This leads to field correlation function for uniform shear flow (neglecting diffusion) [116]

$$
g_{1}(t)=\sum_{n=1}^{\infty} G_{n}\left(x_{i}, x_{o}, l^{*}\right) \exp \left[-\frac{1}{15} n k^{2} l^{* 2} \dot{\gamma}^{2} t^{2}\right] .
$$

For spatially uniform shear flow, the distortion of the path vectors introduced by relative motion is independent of the light path. When relative motion is not spatially homogeneous, the distortion of the path vectors acquires a dependence on the light path through the medium. In particular, the dephasing of the field correlation function will acquire a dependence on the probability that light paths transit particular heterogeneities. In a series of papers, Bicout and co-workers [90, 89] [111 considered the effects of a onedimensional heterogeneity in the strain rate profile $\dot{\gamma}(x)=\partial v_{y}(x) / \partial x$ in an infinite planar slab of thickness $L$ with surface normal $\hat{\mathbf{i}}$ and showed that dephasing expected was given by

$$
\left\langle\Delta \phi_{n}^{2}(t)\right\rangle=\frac{2}{15} n k^{2} l^{* 2} t^{2} \int \dot{\gamma}^{2}(x) \rho_{n}\left(x_{o}, x_{i}, x, l^{*}\right) d x
$$

where $\rho_{n}\left(x_{o}, x_{i}, x, l^{*}\right)$ is a propagator that describes the probability that light diffusing 
from $x_{i}$ to $x_{o}$ will pass through some intermediate point $x$. Explicitly [89]

$$
\begin{gathered}
\rho_{n}\left(x_{i}, x, x_{o}, l^{*}\right)=\frac{2}{L} \frac{\sum_{m=1}^{\infty} \sin \left[\frac{m \pi x_{i}}{L}\right] \sin \left[\frac{m \pi x_{o}}{L}\right] \sin ^{2}\left[\frac{m \pi x}{L}\right] \exp \left[-m^{2} \frac{n}{n_{0}}\right]}{\sum_{p=1}^{\infty} \sin \left[\frac{p \pi x_{i}}{L}\right] \sin \left[\frac{p \pi x_{o}}{L}\right] \exp \left[-p^{2} \frac{n}{n_{0}}\right]} \\
+\frac{2}{L} \frac{\sum_{m=1}^{\infty} \sum_{s=1 \neq m}^{\infty} \sin \left[\frac{m \pi x_{i}}{L}\right] \sin \left[\frac{s \pi x_{o}}{L}\right] \sin \left[\frac{m \pi x}{L}\right] \sin \left[\frac{s \pi x}{L}\right] \frac{\exp \left[-m^{2} n / n_{0}\right]-\exp \left[-s^{2} n / n_{0}\right]}{s^{2}-m^{2}}}{\sum_{p=1}^{\infty} \sin \left[\frac{p \pi x_{i}}{L}\right] \sin \left[\frac{p \pi x_{o}}{L}\right] \exp \left[-p^{2} \frac{n}{n_{0}}\right]}
\end{gathered}
$$

which leads to a field correlation for one-dimensional non-uniform shear flow (neglecting diffusion)

$$
g_{1}(t)=\sum_{n=1}^{\infty} G_{n}\left(x_{i}, x_{o}, l^{*}\right) \exp \left[-\frac{1}{15} n k^{2} l^{* 2} \int \dot{\gamma}^{2}(x) \rho_{n}\left(x_{o}, x_{i}, x\right) d x t^{2}\right] .
$$

This reduces to Eqn. 7.1 when strain rate is uniform.

The behaviour of the propagator $\rho_{n}\left(x_{i}, x, x_{o}, l^{*}\right)$ is examined using simulation in Fig. 7.2. Here, test particles representing the light path in a turbid medium diffuse on a grid from an incident point at $x_{i}$ to a detection point at $x_{o}$. In contrast to the earlier simulation, only diffusion steps that settle at $x_{o}$ are co-added. The simulated behaviours shown in Fig. $7.2 \mathrm{i}$ and iii are very comparable to the analytic results calculated according to Eqn. 7.3 shown in Fig. $7.2 \mathrm{v}$ and vi. Once the propagators $G_{n}$ and $\rho_{n}$ have been determined, $g_{1}(t)$ can be calculated for an arbitrary strain rate profile by numerical integration.

In both the cylindrical-Couette and the cone-plate geometries, the shear stress varies in a known way across the gap. If the flow curve describing the constitutive relation between stress and strain rate in steady-state flow conditions is known, the local shear $\dot{\gamma}(r)$ rate can be determined across the gap from the known local shear stress variation. The empirical Cross model [7] is commonly used to describe the constitutive relation between shear stress and strain rate in shear thinning polymer solutions. This model gives the stress as [7]

$$
\sigma=\dot{\gamma}\left[\frac{\eta_{0}-\eta_{\infty}}{1+(c \dot{\gamma})^{n}}+\eta_{\infty}\right]
$$

Here $\eta_{0}$ and $\eta_{\infty}$ are asymptotic viscosities at low and high strain rate, $n$ is a dimensionless constant and $c$ is a constant with dimensions of time. For certain combinations of these parameters, the Cross model predicts non-monotonic behaviour. It is commonly assumed that the 'underlying' constitutive relation in shear banding wormlike micelles solutions is non-monotonic but that this constitutive behavior is mechanically unstable. For apparent strain rates in this unstable region of the flow curve, the stress plateaus and the fluid partitions into shear bands [73. Motivated by this observation, it is assumed here that this non-monotonic behaviour is not physical in steady-state flow conditions and the Cross model is modified by tying the upper and lower branches of the flow curve together at a 

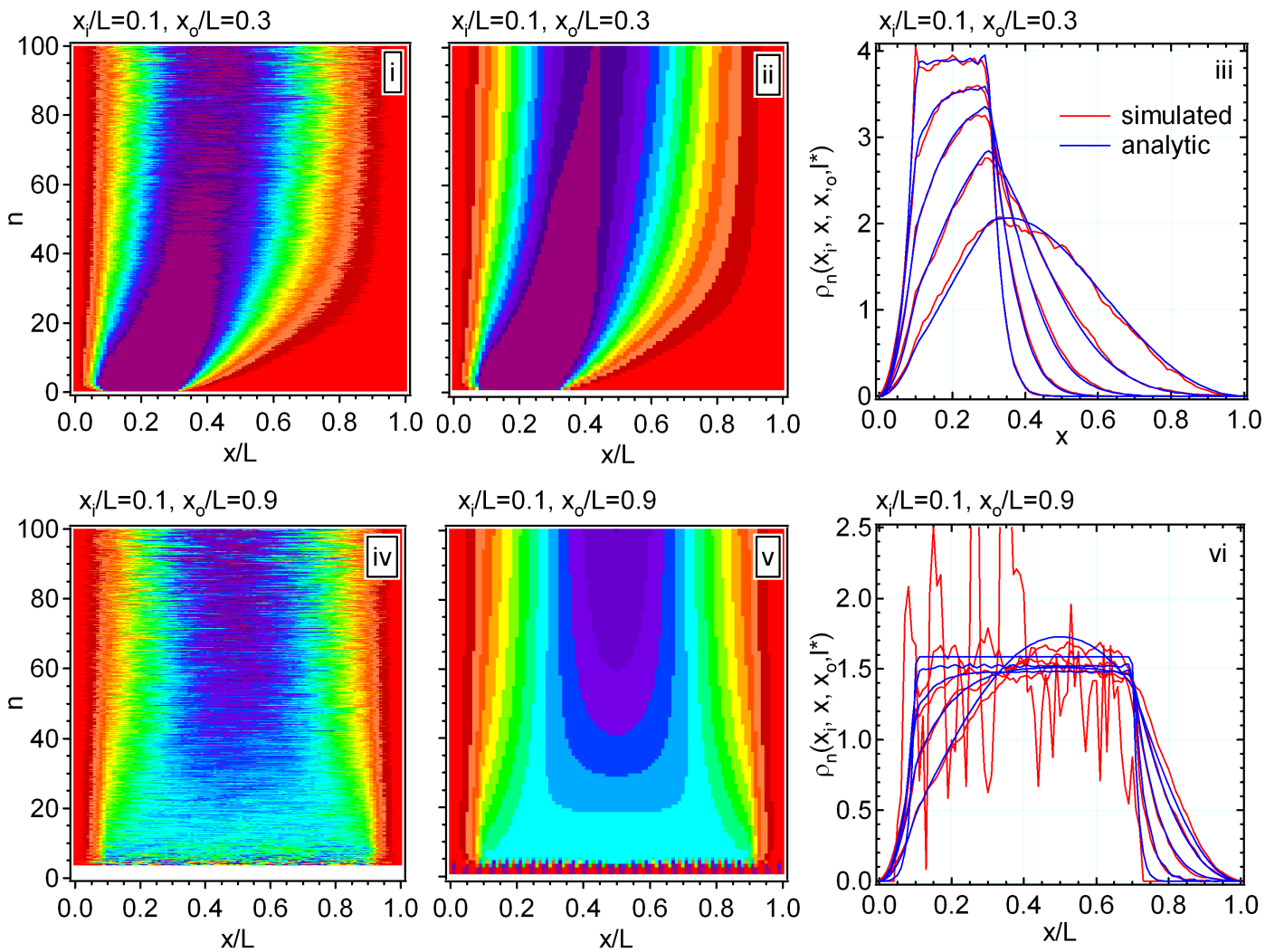

Figure 7.2: i and iii) Simulated and ii and iv) analytic propagators $\rho_{n}\left(x_{i}, x, x_{o}, l^{*}\right)$ with $\mathrm{v}$-vi) direct comparison. 
constant stress. The constant stress is chosen to be an average of the local minimum and maximum of the non-monotonic flow curve.

To investigate the effect of spatial heterogeneities in strain rate on the DWS correlation, the local strain rate variation predicted by the modified Cross model was calculated for a cylindrical-Couette geometry. This strain rate variation is used to calculate the correlation according to Eqn. 7.4. In the calculations, it was assumed that the cylindricalCouette has dimensions $r_{i}=24 \mathrm{~mm}$ and $r_{o}=25 \mathrm{~mm}$ for a gap $L=1 \mathrm{~mm}$. The local curvature of the geometry is small and is treated as an infinite slab in the optical calculations. Physical values of $k=2 \pi /\left(633 \times 10^{-9} / 1.33\right) \mathrm{m}^{-1}, l^{*}=0.2 \mathrm{~mm}$ and $\gamma_{0}=1.7$ were used. $\gamma_{0}$ was measured experimentally as $\gamma_{0} \approx 1.7$ for a glass-water interface in [11]. $\left|g_{1}(t)\right|^{2}$ is calculated for log-spaced increments in apparent strain rate $\dot{\gamma}$ defined

$$
\dot{\gamma}=\frac{r_{i}}{r_{o}-r_{i}} \int_{r_{i}}^{r_{o}} \frac{\dot{\gamma}(r)}{r} d r \approx \frac{v}{d}
$$

where $v$ is the linear velocity of the inner wall and $d=L$ is the gap size for three flow curves $(\mathrm{F} 1, \mathrm{~F} 2, \mathrm{~F} 3)$ and for three optical configurations: backscattering from the outer wall of the cylindrical-Couette (C1-C3), backscattering from the inner wall of the Couette (C4-C6) and transmission through the outer wall of the Couette $(\mathrm{C} 7-\mathrm{C} 9)$. These calculations are shown in Fig. 7.3 C1-C9. As $\dot{\gamma}$ is increased, the correlation advances leftwards on the loglinear plot. The local strain rate variation produced by the 'weakly' shear thinning fluid (F1) does not significantly perturb the correlation $(\mathrm{C} 1, \mathrm{C} 4, \mathrm{C} 7)$. For the 'strongly' shear thinning fluid (F2) the effect on the correlation is more significant (C2,C5,C8). Flow curve (F3) contains a stress plateau. When shear stress in the gap straddles the stress plateau, the fluid partitions into bands of low strain rate $\left(\dot{\gamma}_{L O}\right)$ near the outer wall and high strain rate $\left(\dot{\gamma}_{H I}\right)$ near the inner wall according to the lever rule $\dot{\gamma}=x \dot{\gamma}_{L O}+(1-x) \dot{\gamma}_{H I}$. The local strain rate variation produced during shear banding significantly changes the correlation (C3,C6,C9). This effect should be observable.

\subsection{Experimental}

\subsubsection{Set-up}

Optical measurements were made in cylindrical-Couette, cone-plate and parallel-plate flow geometries. Fig. 7.4 i shows the optical set-up for measurements made using cylindricalCouette geometry. Light from a polarised $15 \mathrm{~mW}$ HeNe laser (L, Melles-Griot) is directed through a beam expander (BE) and though a variable neutral density filter (F) into a homemade optical Couette geometry $\left(\mathrm{CC}, r_{i}=24 \mathrm{~mm}, r_{o}=25 \mathrm{~mm}\right.$, length=60 mm) mounted on an TA AR2000 stress controlled rheometer (R, TA Instruments) used in strain controlled mode. The surface of the cylindrical-Couette bob was blackened to prevent back reflections. After filtering by a polariser $(\mathrm{P})$ which was crossed with the laser, the backscattered light was collected by a GRIN objective and directed through a fibre optic beam splitter (BS, FONT) to 2 photomultipliers (PM, correlator.com PMT-120-OP). The signal from 

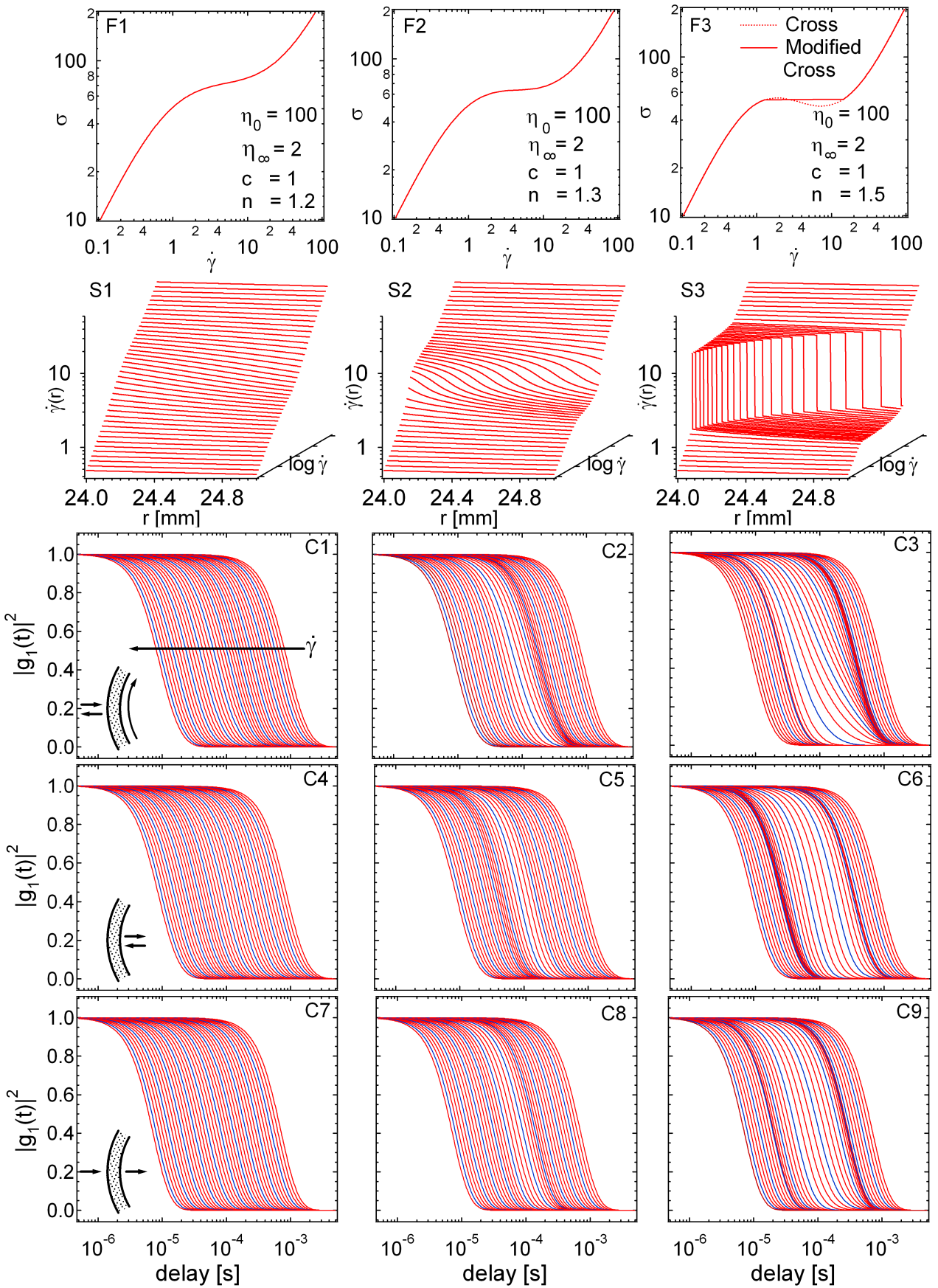

Figure 7.3: Flow curve according to the modified Cross model in three cases (F1, F2, F3). Associated strain rate variation for the flow curves above for a cylindrical-Couette geometry described in text (S1, S2, S3). Corresponding DWS correlations for the optical configuration shown inset and optical conditions discussed in text (C1-C9). 

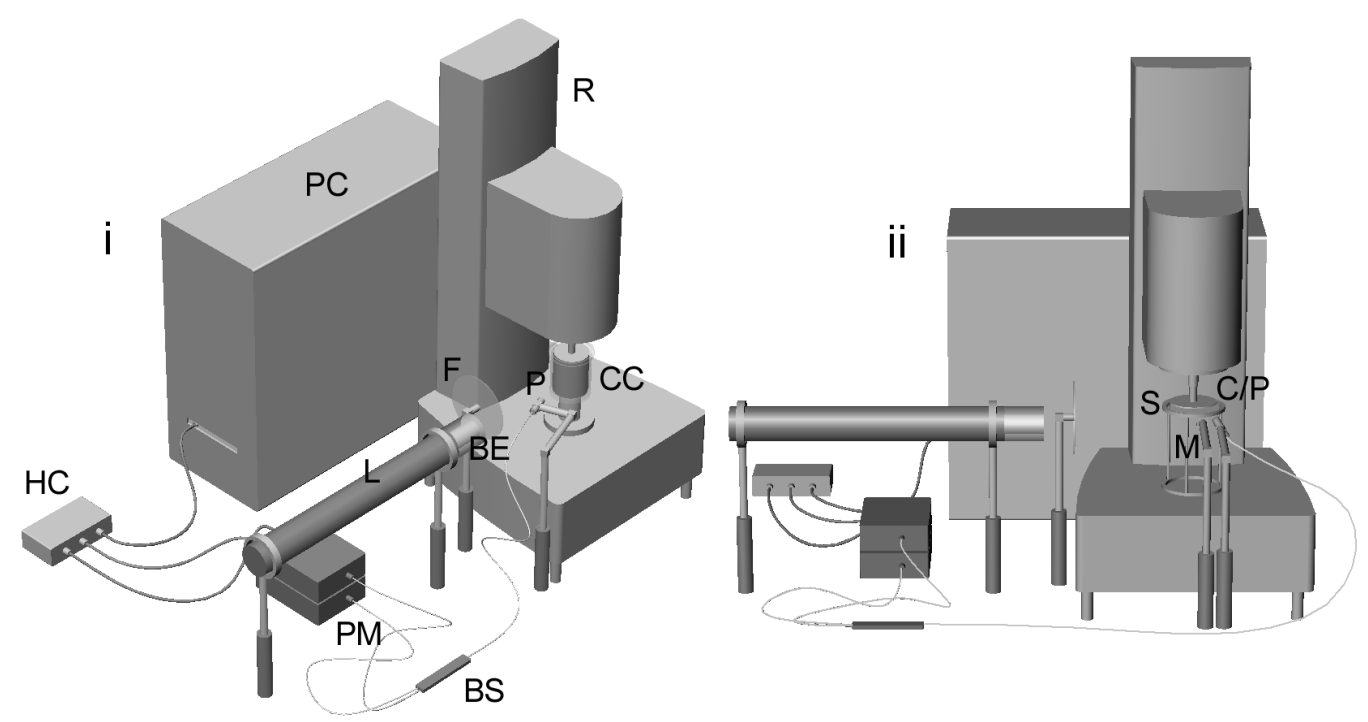

Figure 7.4: Experimental set-up for the i) cylindrical-Couette geometry and an alternative configuration used for the ii) cone-plate and parallel-plate geometries.

the photomultipliers was cross-correlated at a hardware correlator ( $\mathrm{HC}$, correlator.com Flex-02-12) and data were transmitted to the host computer (PC) for analysis. The dimensions of the cylindrical-Couette geometry were chosen to match the dimension of geometry used in [27].

Fig. 7.4 ii shows the optical set-up for measurements made using cone-plate and parallel-plate geometries in the backscattering configuration. Here a transparent, stage mounted $(\mathrm{S})$ pane of glass forms the bottom plate of the cone-plate or parallel-plate geometry $(\mathrm{C} / \mathrm{P})$. Light is reflected by a front reflecting gold mirror $(\mathrm{M})$ through this pane into the sample. The plane of polarisation of the laser is aligned with the plane of incidence of the mirror to ensure that the reflected light is linearly polarised. Backscattered light is collected after filtering with a polariser crossed with the laser from below the plate. By repositioning the laser, mirror and collection optics, backscattering measurements from the top surface of the sample and transmission measurements were possible. For the cone-plate measurements, a blackened, factory, $60 \mathrm{~mm}$ diameter $\theta=4$ deg cone or a transparent, homemade $60 \mathrm{~mm}$ diameter $\theta=4$ deg factory cone was used. Backscattered (or transmitted) light was collected from the bottom (or top) surface at a distance of 18 $\mathrm{mm}$ from the axis of rotation. In the parallel-plate measurements, a blackened, factory, 40 $\mathrm{mm}$ diameter disc was used with a plate-plate separation of $d=1.5 \mathrm{~mm}$. Both collection optics and mirror were mounted on a translation stage - this allowed measurement of the backscattered light as a function of radial position. All optical measurements were made at $T=22{ }^{\circ} \mathrm{C}$ in a temperature regulated room. 

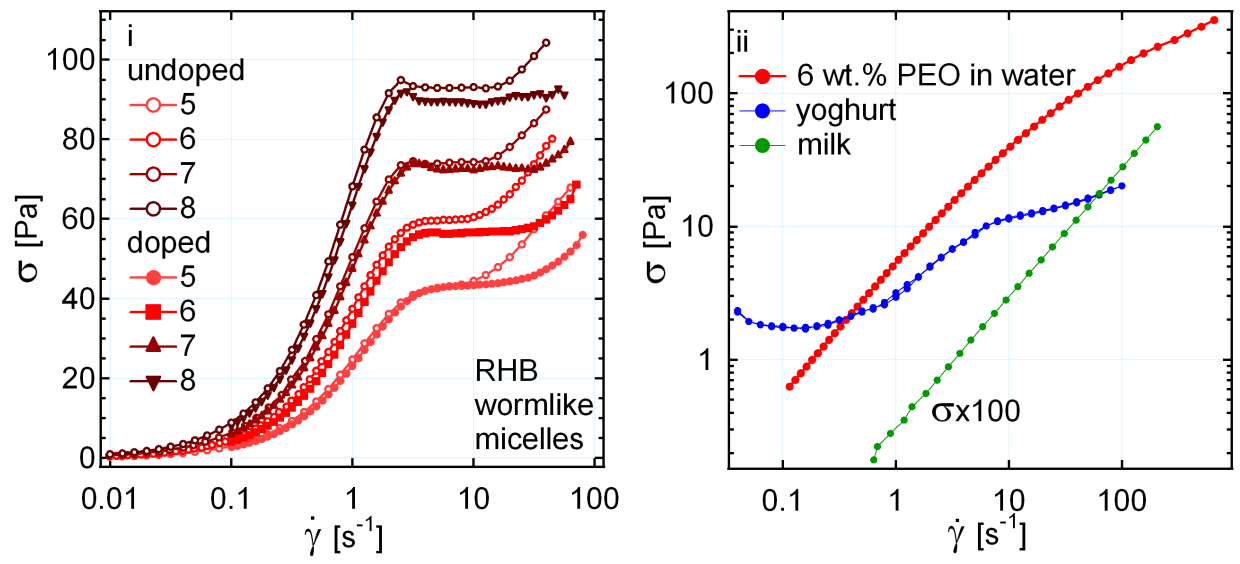

Figure 7.5: i) Nonlinear rheometry of the doped and undoped RHB samples and ii) PEO, milk and yoghurt samples.

\subsubsection{Samples}

RHB wormlike micelles solutions with concentration $\phi_{\mathrm{CPCl}+\mathrm{NaSal}}=5,6,7$ and 8 wt. $\%$ were examined using this 'flow-DWS' technique. A stress plateau was not observed in the flow curve of the $\phi=5$ and $6 \mathrm{wt} \%$ samples in Chapter 5 - these samples were expected to shear thin. A stress plateau was observed in the $\phi=7$ and $8 \mathrm{wt} \%$ samples - these samples were expected to shear band. To produce multiple scattering in these transparent solutions, the samples were doped to approximately $1 \mathrm{wt} . \%$ with washed monodisperse 1 $\mu \mathrm{m}$ diameter latex spheres (Polyscience Inc.). Fig. 7.4 i compares the steady-state flow curve of undoped wormlike micelles solutions with solutions doped with latex spheres. Both measurements were made with a $1 \mathrm{deg} 40 \mathrm{~mm}$ cone-plate. In the undoped case the $\phi=5$ and 6 wt.\% samples showed shear thinning. A stress plateau was observed in $\phi=7$ and 8 wt.\% samples. The rheology of the doped samples showed differences. Most obviously, the thinning/plateau region of the samples was extended. The doped $\phi=6$ wt.\% sample appeared to show some evidence of a stress plateau. At a concentration of approximately $1 \mathrm{wt} . \%$ latex spheres, it would not be expected that hard sphere interactions would significantly affect the rheology. This difference is therefore attributed to a reduction in concentration of surfactant (and change in the relative concentration of surfactant to counter-ion) in solution due to adsorption of surfactant onto the surface of the latex spheres.

A semi-dilute polymer solution composed of $6 \mathrm{wt} . \%$ 600k polyethylene oxide (PEO) in water, a homogenised milk sample (Pams, $3.3 \%$ total fat) and a stirred yoghurt sample (Biofarm, 4.3\% total fat) were also examined using flow-DWS. The PEO solution was doped to approximately $1 \mathrm{wt} . \%$ with washed monodisperse $1 \mu \mathrm{m}$ diameter latex spheres (Polyscience). The milk and yoghurt were not doped - here the intrinsic turbidity of the 
medium was exploited. As illustrated in Fig. 7.5 ii the PEO solution shows weak shear thinning and milk is a low viscosity Newtonian fluid. The yoghurt sample shows a stress plateau at low strain rates. Controlled stress measurements indicated that this plateau extended back to $\dot{\gamma}=0$. This indicates that there is a minimum stress or yield stress that must be applied before the fluid will flow and that the yoghurt is a yield stress fluid.

\subsection{Measurements and analysis}

\subsubsection{Cylindrical-Couette geometry}

Backscattering flow-DWS was used to examine the four RHB wormlike micelles samples in the cylindrical-Couette flow geometry. The strain rate was stepped in intervals of 20 steps per decade until fracture occurred, with a dwell time of $60 \mathrm{~s}$ at each strain rate. Correlations were recorded every $5 \mathrm{~s}$. A single correlation measured in the last $10 \mathrm{~s}$ at each strain rate is reported in Fig. 7.6. Temporal stability of the correlation is illustrated in two cases in Fig. 7.6 v-vi. This figure shows the individual correlation acquired over the $5 \times 12=60 \mathrm{~s}$ interval at each strain rate. The first and last correlation in the interval, which may overlap the step in apparent strain rate, has been excluded. No systematic fluctuation of the correlation was observed suggesting that stable shear flow is established in the fluid in less than $5 \mathrm{~s}$.

The correlations observed here show the behaviour predicted in Fig. 7.3 C2 and C3. To begin quantifying the observations, the data shown in Fig. 7.7 were fitted to the uniform shear flow model described by Eqn. 7.1. The fitted strain rate $\dot{\gamma}_{f i t}$ is shown in Fig. 7.7. It was assumed that $L=1 \mathrm{~mm}, l^{*}=0.18 \mathrm{~mm}$ (this $l^{*}$ was chosen as a best fit for all data presented here prepared using a stock 1 wt.\% suspension of $1 \mu \mathrm{m}$ spheres) and $\gamma_{0}=1.7$. At low strain rates, the fluid is Newtonian and the fitted strain rate is proportional to the strain rate. Experimentally, it is found that $\dot{\gamma}_{f i t}=0.95 \dot{\gamma}$. A slightly smaller value is expected. The instrument applies a strain rate $\dot{\gamma}=v / d$ where $v$ is the linear velocity of the moving inner wall. For a Newtonian fluid, the local strain rate is lower nearest the outer wall because of the slight decrease in stress across the gap. Backscattering measurements preferentially sample motion nearest the incident surface, which is the outer wall in this case. It is observed that the fitted strain rate at higher strain rate is again proportional to the strain rate with a similar constant of proportionality to that observed at low strain rates. Shear induced turbidity was observed in Chapter 4. It could be expected that this turbidity would reduce the $l^{*}$ value. This would reduce the apparent (fitted) strain rate $\dot{\gamma}_{f i t}$ if not corrected for. A $6 \%$ reduction in the constant of proportionality is observed - this change in $l^{*}$ is small and will be neglected here.

In Fig. 7.3 the correlations are calculated from the flow curve - it seems possible that the flow curve (up to a constant of proportionality) could be determined from the correlation by fitting the data. Fig. 7.7 ii shows a fit to the parameters $\eta_{\infty}, c$ and $n$ of the modified Cross model. The final parameter $\eta_{0}$ was determined separately from mechanical measurements. As seen in Fig. 7.7 i-iv the fitted correlation fairly successfully reproduces 

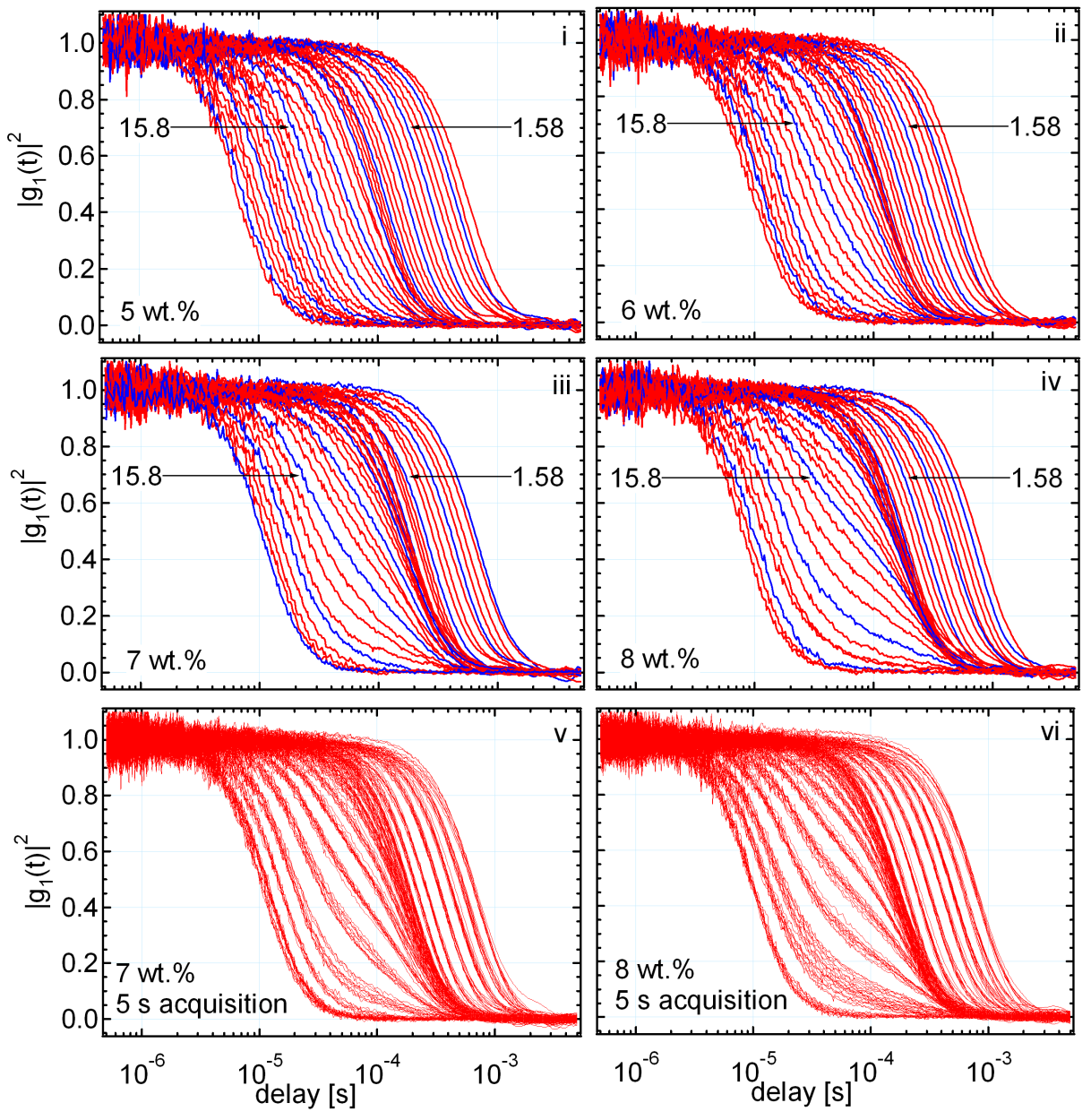

Figure 7.6: i-iv) Backscattering flow-DWS measurements of $\phi=5,6,7,8$ wt.\% RHB wormlike micelles during a stepped strain rate test. Correlations measured at $\dot{\gamma}=10^{-0.2}$, $10^{0}, 10^{0.2}, 10^{0.4} \ldots \mathrm{s}^{-1}$ are shown with a blue line. $\mathrm{v}$-vi) Temporal stability of the flow-DWS correlations of selected samples. 
the measurements. In Fig. $7.7 \mathrm{v}$ the optically determined flow curve is compared to the mechanical one. The optical and mechanical flow curves match very well for the $\phi=6,7$ and 8 wt. $\%$ samples. The optical flow curve for the $\phi=5 \mathrm{wt} . \%$ sample is less comparable.

The data in Fig. 7.6 was reanalysed using a different approach. Instead of trying to relate the measured correlations to the rheological behaviour, an attempt was made to extract the strain rate profile by fitting each correlation separately. It was assumed that the velocity profile at each strain rate could be described by second order rational Bézier spline [117] defined as

$$
\left[\begin{array}{c}
V(t) \\
X(t)
\end{array}\right]=\frac{1}{\sum_{i=0}^{n} W_{i} B_{n, i}(t)} \sum_{i=0}^{n} W_{i} B_{n, i}(t) \mathbf{P}_{i}
$$

where $n=2$,

$$
P_{0}=\left[\begin{array}{l}
0 \\
0
\end{array}\right], \quad P_{1}=\left[\begin{array}{l}
V_{1} \\
X_{1}
\end{array}\right], \quad P_{2}=\left[\begin{array}{l}
1 \\
1
\end{array}\right]
$$

and $B_{n, i}$ are the Bernstein polynomials defined

$$
B_{n, i}(t)=\frac{n !}{i !(n-i) !} t^{i}(1-t)^{n-i} .
$$

$V=v /\left(v_{o}-v_{i}\right)$ is the reduced velocity and $X=x /\left(x_{o}-x_{i}\right)$ is the reduced position in the gap. With the weighting parameters $W_{0}=1$ and $W_{2}=1$ fixed and the parameters $v_{o}$ and $v_{i}$ known (which is only true if no-slip boundary conditions are assumed), Eqn. 7.7 is a function of the parameters $W_{1}, V_{1}$ and $X_{1}$. Fig. 7.8 i shows $V(t)$ versus $X(t)$ for three combinations of $X_{1}, V_{1}$ and for various $W_{1}$ to give a reduced velocity profile. These data are interpolated and differentiated to give the associated reduced strain rate profile shown in Fig. 7.9 ii. The individual correlations were fitted to determine the strain rate profile in the gap according to the three parameter Bézier spline model. Typical fits are shown in Fig. 7.9 iii-iv. The agreement is far better than that observed in Fig. 7.7. This is not at all surprising as here there are three free parameters per correlation.

The strain rate profiles calculated from the modified Cross model and Bézier spline fit are compared in Fig. 7.9. The strain rate predicted by the Bézier spline representation is reasonably comparable justifying the approach.

The strain rate dependence of the correlation function depends only on the size, concentration, and polydispersity of the scattering centres through the mean free transport path $l^{*}$. This is demonstrated in Fig. 7.10. Fig. 7.10 i shows flow-DWS measurements for an $8 \mathrm{wt} \%$ wormlike micelles solution doped to $2.5 \mathrm{wt} \%$ with a polydisperse $\mathrm{SiO}_{2}$ powder (Sigma, particle size 0.5-10 $\mu \mathrm{m}, 80 \%$ between 1-5 $\mu \mathrm{m}$ ) measured in the cylindrical-Couette geometry using the protocol discussed earlier. The correlations show similar behaviour to that observed for the suspension of monodisperse latex spheres. Fig. 7.10 ii shows flowDWS measurements for a homogenised milk sample in the same geometry. Milk is a 

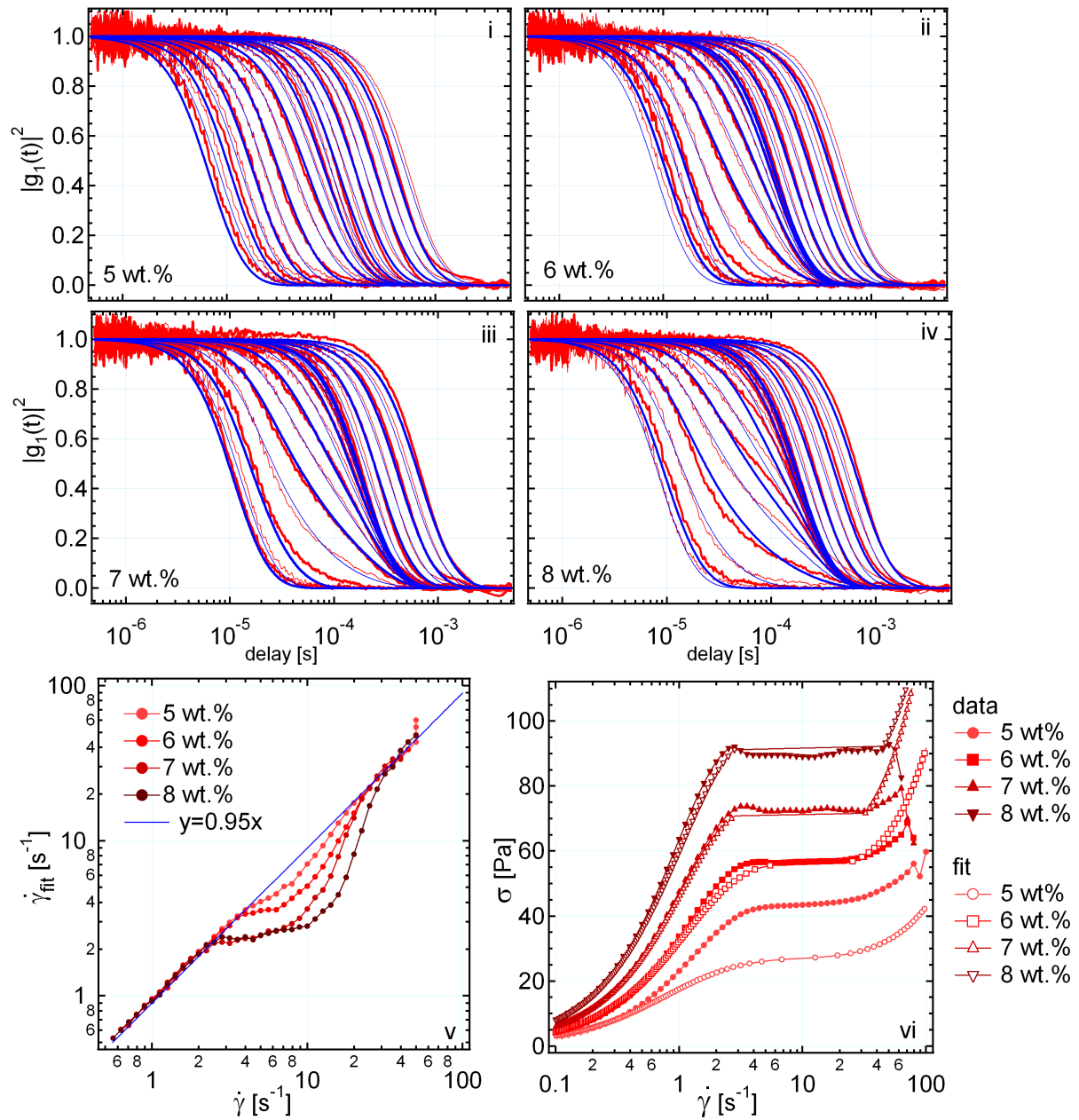

Figure 7.7: i-iv) Fit to the modified Cross model. iv) Fitted strain rate from a fit to the data shown in Fig. 7.6 to the uniform shear flow model given by Eqn. 7.1. vi) Flow curves determined optically using a fit to a three parameter modified Cross model with comparison to the mechanically determined flow curve. 

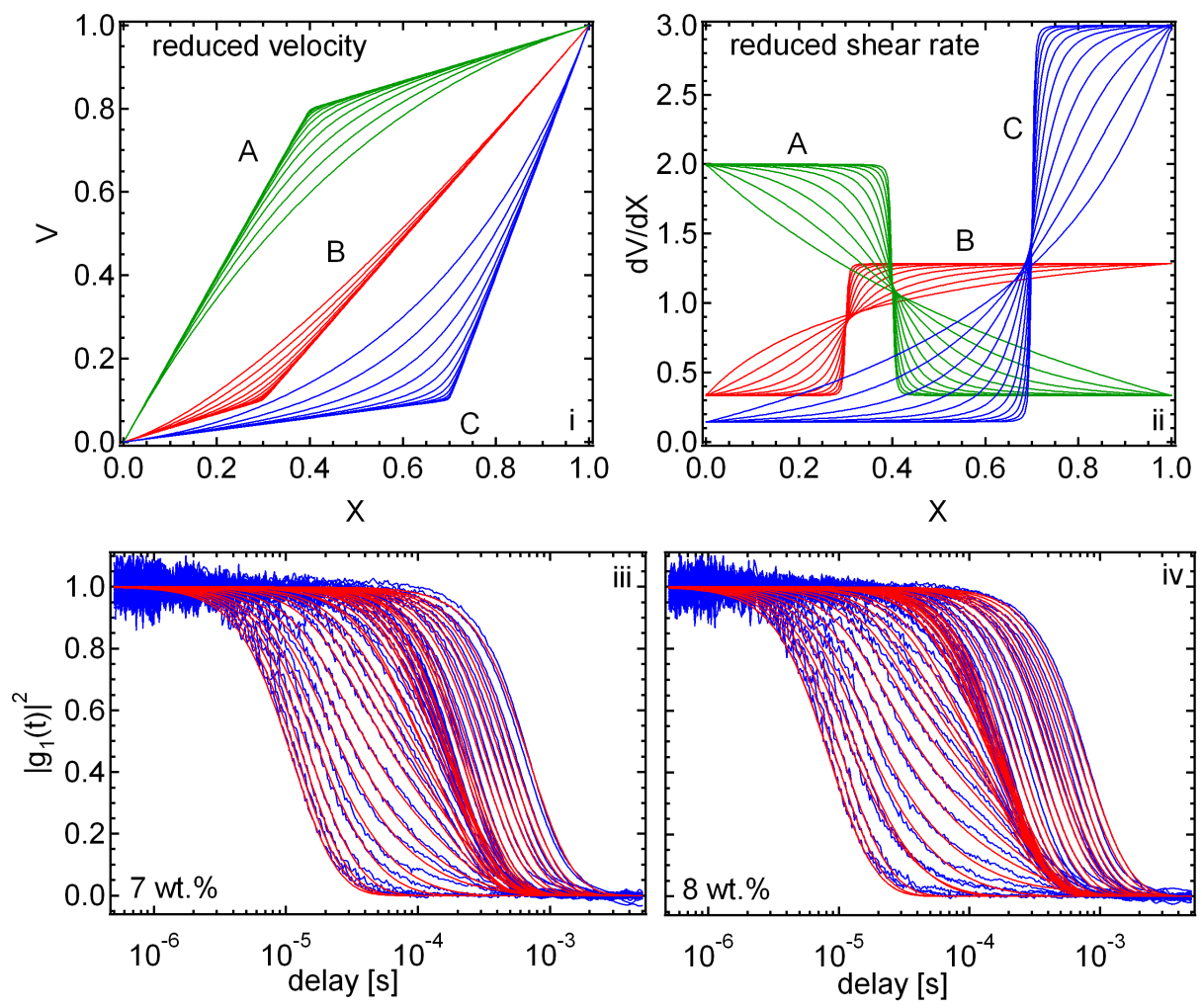

Figure 7.8: i) Three parameter rational Bézier spline representations of the reduced velocity profile in the gap for three combinations of $X_{1}$ and $V_{1}$ (corresponding to families A,B and $\mathrm{C}$ ) for various $W_{1}$. ii) The associated strain rate profile calculated by interpolating and differentiating the data in i. ii-iv) Fit to data shown in Fig. 7.6 to the Bézier spline model (experimental data in blue, fitted data in red). 

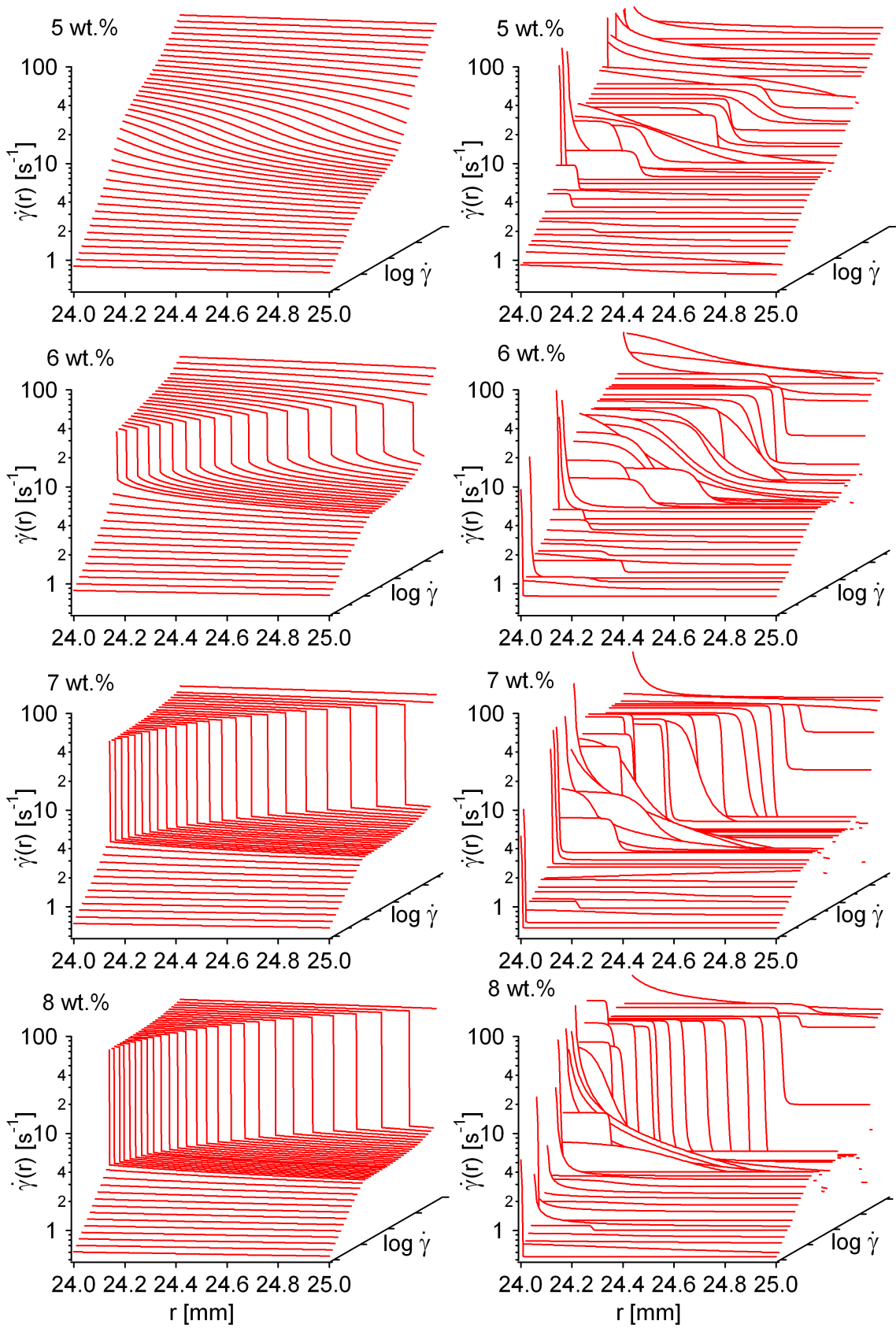

Figure 7.9: A comparison between the strain rate profiles over a range of apparent strain rates for wormlike micelles solutions at different concentrations. The profiles on the left hand side were derived from the fitted optical flow curves, while those on the right hand side were inferred from the Bézier spline fit. Note that the flow curve calculation incorporates the entire set of data from the full range of apparent strain rates at each concentration, and this, of necessity, produces a self consistent and smoothly varying set of profiles. By contrast, the Bézier spline method allows for an independent strain rate profile fit to each individual apparent strain rate. 

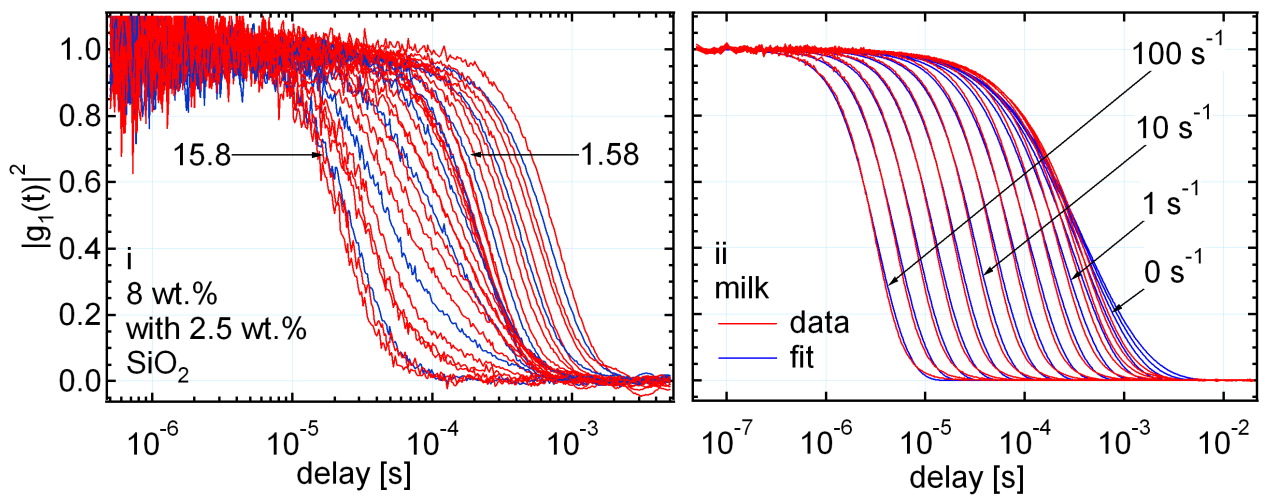

Figure 7.10: Flow-DWS measurement of a i) 2.5 wt.\% suspension of a polydisperse silica powder in an 8 wt.\% RHB wormlike micelles solution and ii) milk during a stepped strain rate test demonstrating that monodispersity is not required for flow-DWS.

polydisperse dispersion of fat droplets and casein micelles in water - probe particles are not required in this instance as the fat droplets present in the milk produce the necessary turbidity. Here the data are fitted to

$$
g_{1}(t)=\sum_{n=1}^{\infty} G_{n}\left(x_{i}, x_{o}, l^{*}\right) \exp \left[-\frac{1}{15} n k^{2} l^{* 2} \dot{\gamma}^{2} t^{2}\right] \exp \left[-2 k^{2} D^{*} t\right],
$$

where $D^{*}$ is an effective diffusion constant. At low strain rates the diffusion of the droplets dominates. At higher strain rates the correlations recovers the familiar strain rate dependence.

\subsubsection{Cone-plate geometry}

Backscattering flow-DWS was used to examine the doped 6 wt.\% 600k PEO solution in the cone-plate geometry. The optical configuration is shown schematically in Fig. 7.11 i (inset). The direction of illumination is illustrated with the arrow and $d$ indicates the position of the collection optics. The stress was stepped in intervals of 20 steps per decade until fracture occurred, with a dwell time of $60 \mathrm{~s}$ at each strain rate. Fig. 7.11 i shows data with a fit to the uniform shear flow (Eqn. 7.1$)$ model assuming $L=18 \times \tan (4 \pi / 180) \approx 1.25$ $\mathrm{mm}, l^{*}=0.18 \mathrm{~mm}$ and $\gamma_{0}=1.7$ to determine $\dot{\gamma}_{f i t}$. In the cone-plate geometry, the stress varies through the gap by a factor $\sigma_{\phi, \theta}(90 \times \pi / 180) / \sigma_{\phi, \theta}([90-4] \times \pi / 180) \approx 1.005$. Because the stress variation through the gap is far smaller than in the cylindrical-Couette geometry and because the sample shows weak shear thinning, it is expected that the strain rate in the gap will be nearly constant and that $\dot{\gamma}_{f i t}$ is to a good approximation equal to $\dot{\gamma}$. The mechanically applied stress with respect to both the optically and mechanically measured strain rates are plotted with respect to the measured mechanical stress in Fig. 7.11 ii. These two measurements show good agreement. 
The flow curve of the yoghurt sample indicated that it is a yield stress fluid. Like some of the wormlike micelles solutions examined, a yield stress fluid has a stress plateau. However, unlike the wormlike micelles it has no lower Newtonian branch - the plateau continues back to zero strain rate. By the lever rule, it could be expected that the yield stress fluid would divide into bands of strain rate $\dot{\gamma}_{L O}$ and $\dot{\gamma}_{H I}$ with $\dot{\gamma}_{L O}=0$ and with the high shear band forming the high stress region of the flow geometry and expanding out as the apparent strain rate is increased. Fig. 7.11 shows both backscattering and transmission flow-DWS measurements. The transmission measurements through the top and bottom surfaces are very similar whereas the backscattering correlations show marked differences. As already noted, in the backscattering geometry short paths are favoured. Consequently, motion nearest the incident surface is sampled. The difference between the sequence of correlations shown in Fig. 7.11 $\mathrm{i}$ and iii suggests the relative motion is different near the two surfaces of the geometry. Motion is dominated by diffusion near the plate, suggesting that the local strain rate at this surface is small or zero, and by shear near the cone. This immediately suggests shear banding of the form expected for a yield stress fluid. These data are discussed along with NMR velocimetry in [118].

Fig. 7.12 i-iv shows flow-DWS measurements of the doped 5, 6, 7 and 8 wt.\% RHB wormlike micelles in the backscattering configuration and $7.12 \mathrm{v}$-vi and doped 7 and 8 wt.\% samples in the transmission geometry. The strain rate was stepped in logspaced intervals. Note that the correlations measured for the $5 \mathrm{wt} \%$ sample do not show the inflection seen in Fig. 7.6 i. This is to be expected and is a consequence of the difference in the shear stress variation across the gap in the two geometries. The sequence of correlations observed in Fig. 7.12 i-iv resembles the backscattering correlations observed in the cylindrical-Couette geometry. The transmission correlations in Fig. $7.12 \mathrm{v}$-vi are similar to the model correlations calculated in Fig. 7.3 C3 and C9 for a shear banding fluid. These observations suggest that as in the cylindrical-Couette geometry, the high strain rate band expands from the moving surface of the geometry with partitioning given by the lever rule.

Following the procedure outlined in the last section, the four backscattering correlations were fitted to the modified Cross model. The fitted correlations did not satisfactorily match the measured correlations (fit not shown). To understand why the fit was unsatisfactory, the data were fitted to the uniform shear flow model and are compared to a fit to model correlations, shown in Fig. 7.13. The model correlations were calculated assuming that the fluid shear bands according to the lever rule, $\dot{\gamma}=x \dot{\gamma}_{L O}+(1-x) \dot{\gamma}_{H I}$, with a high strain rate band expanding from the moving wall of the geometry for model values $\dot{\gamma}_{L O}=2 \mathrm{~s}^{-1}, \dot{\gamma}_{H I}=20 \mathrm{~s}^{-1}, l^{*}=0.18 \mathrm{~mm}, L=1.25 \mathrm{~mm}$ and $\gamma_{0}=1.7$ in the two optical configuration presented in Fig. 7.12, Fig. 7.13 i shows the fitted strain rate $\dot{\gamma}_{f i t}$ to the model backscattering and transmission correlations. The 400-500 measured correlations for the 5 and $8 \mathrm{wt} . \%$ were fitted to the uniform shear flow model (again assuming $l^{*}=0.18$ $\mathrm{mm} L=1.25 \mathrm{~mm}$ and $\left.\gamma_{0}=1.7\right)$ to determine $\dot{\gamma}_{f i t}$. As seen in Fig. 7.13 iii, for the 5 wt. $\%$ sample $\dot{\gamma}_{f i t}=1.15 \dot{\gamma}$ at lower strain rates increasing to $\dot{\gamma}_{f i t}=1.30 \dot{\gamma}$ at higher strain rates. This is the opposite of the effect expected if shear induced turbidity was reducing $l^{*}$. The constant of proportionality between $\dot{\gamma}_{f i t}$ and $\dot{\gamma}$ at low strain rates was found to vary 

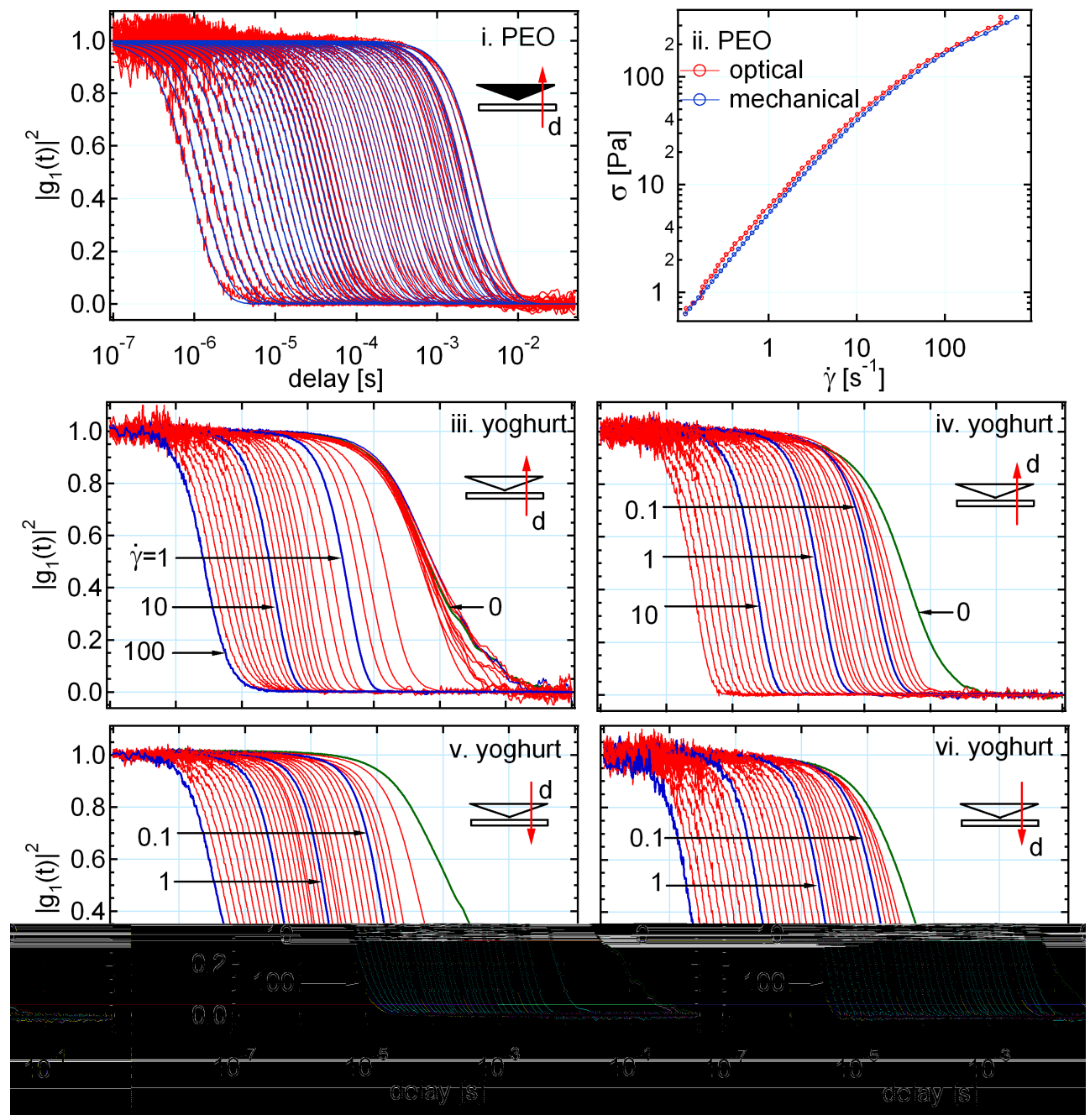

Figure 7.11: i) Backscattering flow-DWS measurements of the 600k 6 wt.\% PEO sample in the cone-plate geometry during a stepped stress test. ii) Optical and mechanical flow curves. iii-vi) Backscattering and transmission flow-DWS measurements of the yoghurt sample during a stepped strain rate test. 

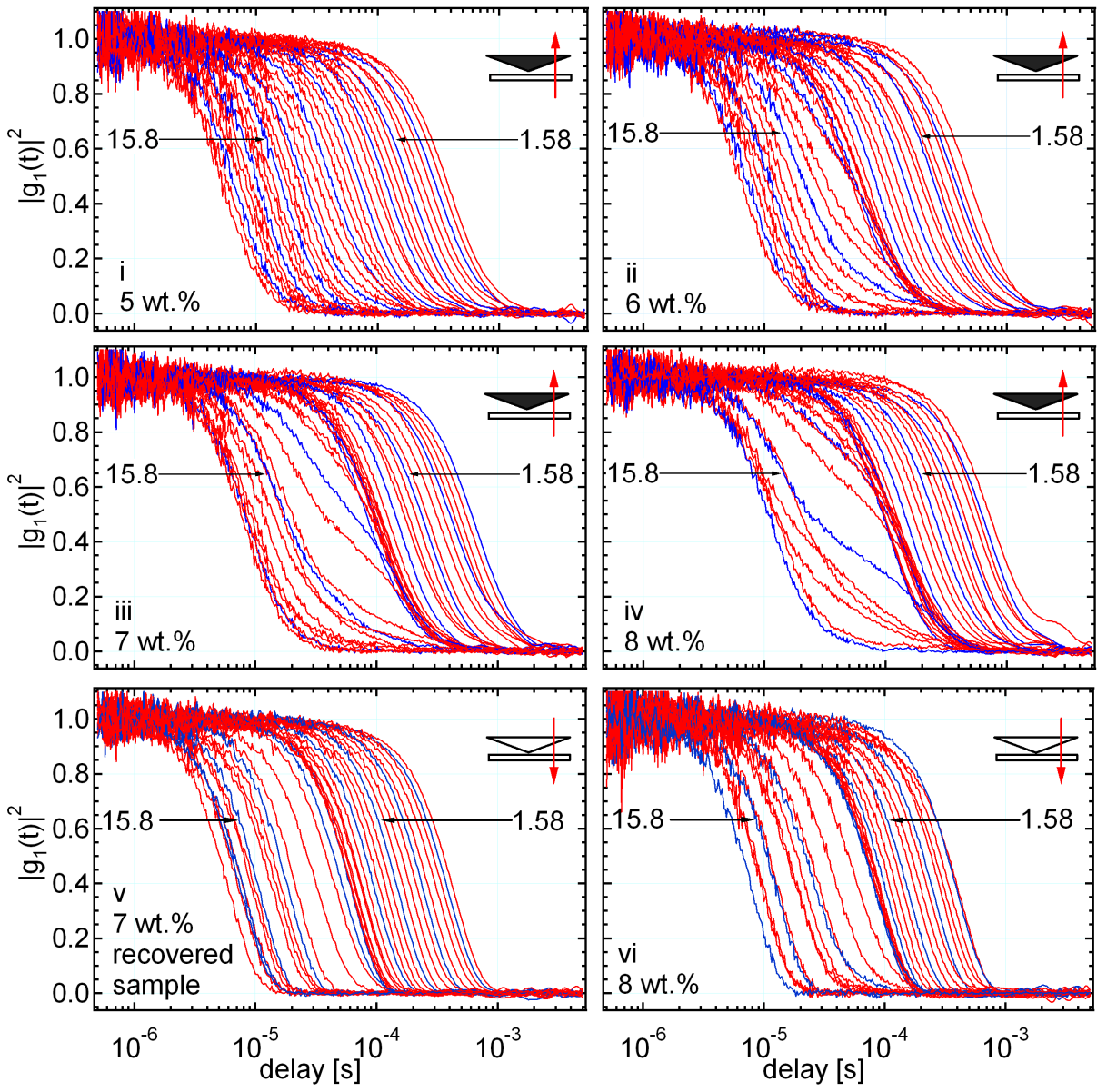

Figure 7.12: i-iv) Backscattering flow-DWS measurements of the 5-8 wt.\% RHB samples and v-vi) transmission measurements of the $7-8$ wt.\% RHB samples in the cone-plate geometry during a stepped strain rate test. Correlations measured at $\dot{\gamma}=10^{-0.2}, 10^{0}$, $10^{0.2}, 10^{0.4} \ldots \mathrm{s}^{-1}$ are shown with a blue line. 
between measurements by about $20 \%$ for the cone-plate geometry. This may be due to slight changes in the alignment of the optics between measurements. The propagator $G_{n}$ and $\rho_{n}$ described by Eqn. 4.78 and Eqn. 7.3 assumes uniform illumination. The intensity distribution of an expanded Gaussian laser beam is Gaussian. The illumination is only (approximately) uniform at the centre of the beam spot. If, because of an alignment error, light was not collected from the centre of the beam spot, illumination is no longer (approximately) uniform. This would modify $G_{n}$ and $\rho_{n}$ affecting the measured correlation.As seen in Fig. 7.13 iv, the fit to the backscattering and transmission correlations suggest that uniform shear flow is present in 8 wt.\% sample up to $\dot{\gamma} \approx 4 \mathrm{~s}^{-1}$. Between $4-5 \mathrm{~s}^{-1}$ $\dot{\gamma}_{f i t}$ is observed to drop by $25 \%$ (A in Fig. 7.13 iv). A similar trend was seen in the 6 and 7 wt.\% samples. From the model fit, it is predicted that $\dot{\gamma}_{f i t}$ is a monotonically increasing function of $\dot{\gamma}$ - the disagreement at A indicates a problem with the model in this instance. One speculative possibility is that a narrow, high strain rate slip band is developing at one or both of the walls. The sensitivity of DWS to relative motion decreases significantly within a distance of $l^{*}$ of the walls of the geometry. $G_{n}$ and $\rho_{n}$ increase quadratically from zero at the walls. If the slip band was sufficiently narrow it could not be directly observed using flow-DWS. However, the presence of slip could be inferred because it would reduce the apparent strain rate in the rest of the gap. The reduction in $\dot{\gamma}_{f i t}$ at A could indicate the development of a slip band. Region B in Fig. 7.13 iv is similar to that shown in region B in Fig. 7.13 i suggesting that following the development of the slip band, a macroscopic high strain rate band develops at the moving wall of the geometry and expands into the gap with increasing $\dot{\gamma}$ according to the lever rule. Fig. 7.13 ii shows the steady-state flow curve measured concurrently with flow-DWS measurements with either a blackened steel (backscattering) or acrylic (transmission) cone. The behaviour is similar to that observed in Chapter 4. An increase in $\sigma$ is observed at $\dot{\gamma}=7.94 \mathrm{~s}^{-1}$. An increase in $\dot{\gamma}_{f i t}$ is seen in the backscattering flow-DWS measurements around $\dot{\gamma}=12 \mathrm{~s}^{-1}$. It is notable that this increase does not directly correlate with the onset of increasing stress.

NMR velocimetry was used previously to examine the flow behaviour of a shear banding wormlike micelles solution in a $\theta_{0}=4 \mathrm{deg}$ cone-plate geometry [31]. This geometry was sealed at the perimeter and measurements were time averaged over several hours. The NMR velocimetry measurements indicated that a high strain rate band formed in the centre of the gap. In a subsequent theoretical study of shear banding in viscoelastic fluids in the cone-plate geometry, Kumar and Larson [119] suggested that the banding observed in [31] may be associated with secondary flow. In Fig. 7.13 v-vi a series of correlations is calculated in the case where a high strain rate band forms mid-gap and expands symmetrically with increasing strain rate with partitioning given by the lever rule. It was assumed that apparent strain rate was stepped in log-spaced intervals and that the optical configuration matched those used experimentally. The measured correlation sequence appears to be inconsistent with the correlation sequence predicted by this model. 

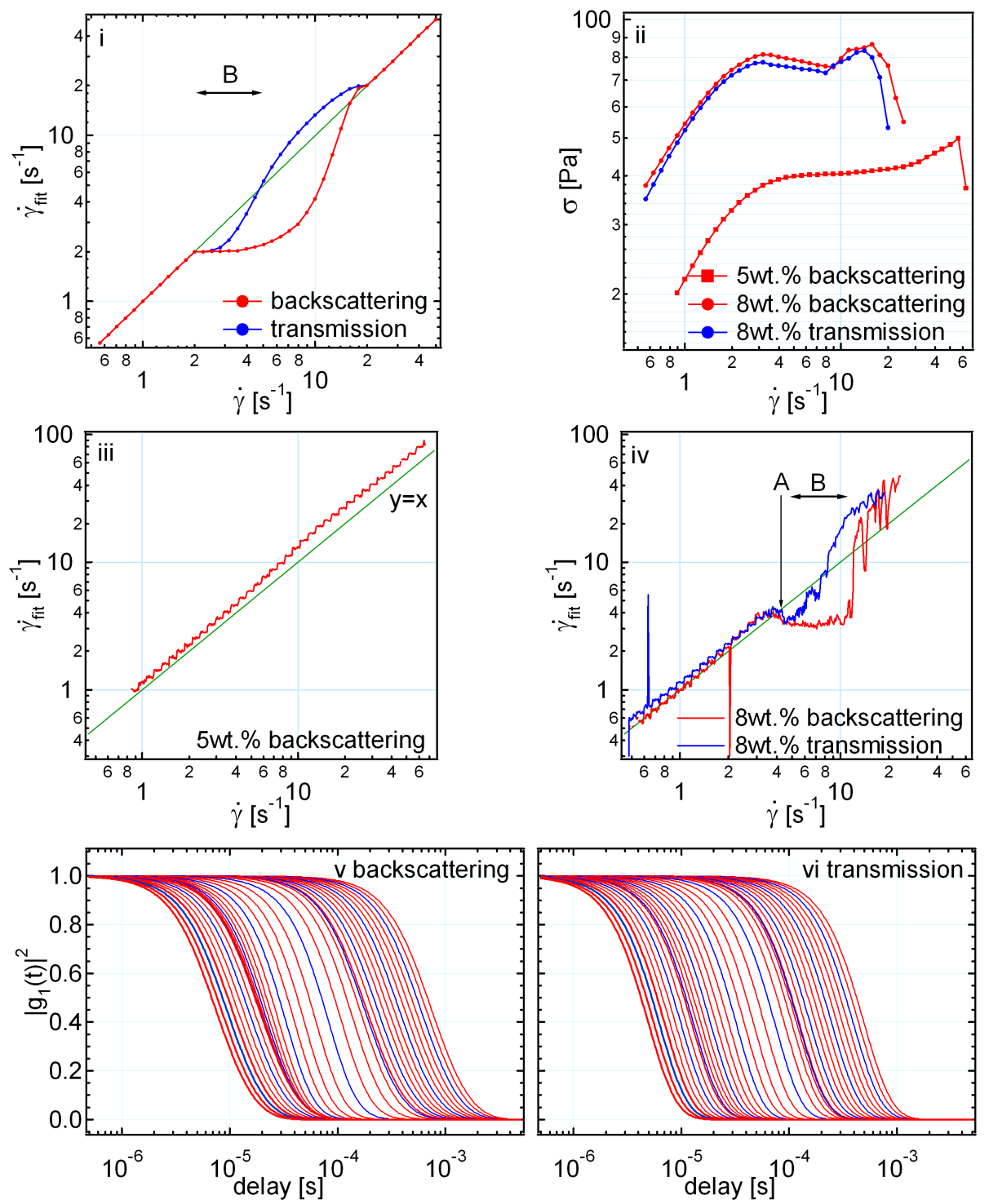

Figure 7.13: i) Fit to the uniform shear flow model of flow-DWS correlations calculated for a model shear banding fluid in both the transmission and backscattering configurations. ii) Flow curve of the $\phi=5$ and 8 wt. $\%$ RHB samples measured during the cone-plate flow-DWS experiment. Fit to the uniform shear flow model of flow-DWS correlations measured in the cone-plate geometry in the iii) backscattering and iv) backscattering and transmission configurations. v-vi) Model flow-DWS correlations in the case where the high strain rate band expands from the centre of the gap. 


\subsubsection{Parallel-plate geometry}

Backscattering flow-DWS was used to examine the doped 6 wt.\% 600k PEO and 5 wt.\% RHB wormlike micelles solution in a $40 \mathrm{~mm}$ parallel-plate geometry with a gapsize $d=1.5$ mm. Fig. 7.14 i shows measured correlations as a function of both radial position, at 0.5 or $1 \mathrm{~mm}$ increments, and angular velocity of the top plate for the $5 \mathrm{wt} . \%$ RHB sample. The correlations at each angular velocity step have been offset for clarity.

As before, data were fitted to the uniform shear flow model with $L=1.5 \mathrm{~mm}$, $\gamma_{0}=1.7$ and $l^{*}=0.18 \mathrm{~mm}$. The fitted strain rate is proportional to the angular velocity and the radius, several $\mathrm{mm}$ out from the centre of the geometry, as is shown in Fig. 7.14 ii. This is demonstrated more clearly in Fig. 7.14 iii. In the parallel-plate geometry $\dot{\gamma}(r)=v / d=r \omega / d$. It might therefore be expected that the characteristic time of the correlation for $\mathrm{r} \approx 0$ would be diffusion limited and unaffected by the applied shear - this was not observed. The diffuse 'scattering volume' probed in DWS depends on optical configuration. The backscattering correlation is dominated by $n=1$ term in Eqn. 4.78 indicating that the radius of the scattering volume is around $l^{*}$. Light collected at the centre rotation at $r=0$ contains a contribution from near the centre $r \neq 0$ where the strain rate is not zero. This radial variation in strain rate could be expected to reduce the characteristic time of the correlation. Outside the central region of the geometry, the fitted strain rate is quite comparable to the apparent strain rate $\dot{\gamma}(r)=r \omega / d$ indicating that the shear flow is uniform.

Fig. 7.15 i shows measured correlations as a function of both radial position, measured at 0.5 or $1 \mathrm{~mm}$ increments, and angular velocity of the top plate for the $8 \mathrm{wt} . \%$ RHB sample. As before, the correlations at each angular velocity step have been offset for clarity. The flow protocol, along with the measured stress, is illustrated in Fig. 7.15 iii. Note that the dwell time at each perimeter strain rate $\dot{\gamma}_{0}$ was either 1200 or $2400 \mathrm{~s}$. The total acquisition time at each of the 20 radial positions measured was either 60 or 120 $\mathrm{s}$. The correlations presented in Fig. $7.15 \mathrm{i}$ are time averaged over the last $50 \mathrm{~s}$ or $110 \mathrm{~s}$ at each radial position - $10 \mathrm{~s}$ was allowed to manually translate the mirror and collection optics. The correlations shown in Fig. 7.15 i are mapped to the surface shown in Fig. 7.15 ii. The black line represents the time required for the correlation to decay $\left|g_{1}(t)\right|^{2}=0.5$ assuming uniform shear flow. These correlations were also fitted to the uniform shear flow model; fitted $\dot{\gamma}_{f i t}$ is shown in Fig. 7.15 v. At $\dot{\gamma}_{0}=3 \mathrm{~s}^{-1}$ the fitted strain rate is directly proportional to applied stain rate. At $\dot{\gamma}_{0}=6 \mathrm{~s}^{-1}$ at a position that corresponds to a local stain rate $\dot{\gamma}(r)=v / d=2.4 \mathrm{~s}^{-1}$ (point A in Fig. 7.15 ii) the correlations show essentially no change with radial position. Similar behaviour is seen at the onset of shear banding in the cylindrical-Couette geometry, suggesting that a high strain rate band may be forming at the moving plate. If a high strain rate band was expanding from the upper surface, it would be expected that the correlation near the perimeter would show reduced $\dot{\gamma}_{f i t}$ because of the intrinsic radial strain rate variation present in this geometry as the high strain rate band comes into the 'field of view' of the diffuse scattering volume. This is not observed in the measurements at $\dot{\gamma}_{0}=9$ and $12 \mathrm{~s}^{-1}$. Loosely, correlations measured at $\dot{\gamma}_{0}=6,9$ and $12 \mathrm{~s}^{-1}$ can be divided into three radial bands. In the central band/disc 

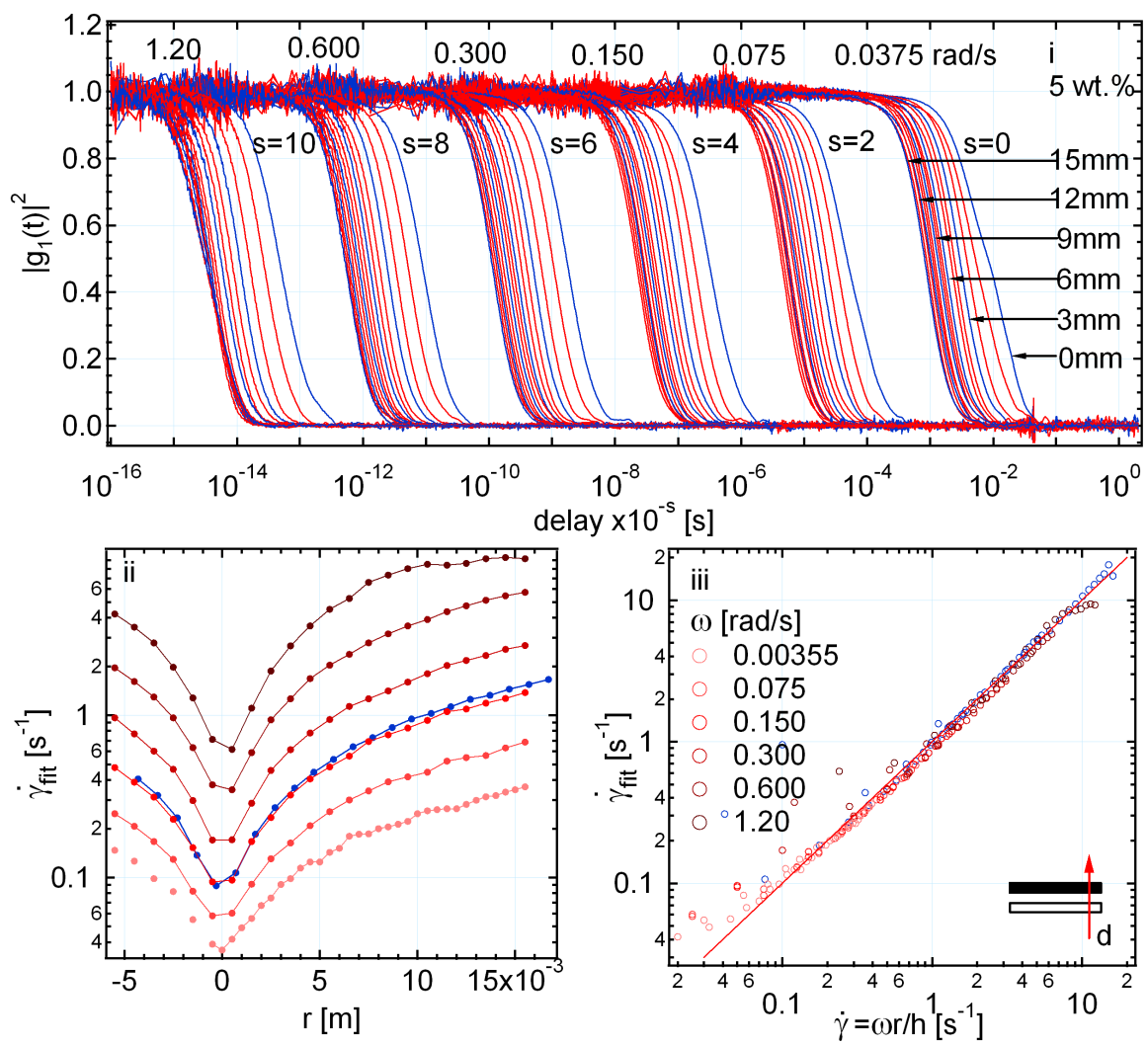

Figure 7.14: i) Backscattering flow-DWS measurements from the 5 wt.\% RHB sample with radial position and angular velocity in the parallel-plate geometry. ii) Fit to the uniform shear flow model and iii) a comparison with the expected local strain rate. Red/blue markers in ii-iii are for the $5 \mathrm{wt} . \% \mathrm{RHB} / 600 \mathrm{k}$ PEO samples. 
the shear flow is 'apparently' uniform. This band/disc expands radially with increasing $\dot{\gamma}_{0}$. Beyond the edge of this disc is a transition band. Correlations in the transition band show the greatest deviation from correlations expected for uniform shear flow and are reminiscent of the correlations observed at higher strain rates in the shear banding wormlike micelles in the cylindrical-Couette geometry. Near the edge of the geometry is a perimeter band which has a roughly constant fitted strain rate $\dot{\gamma}_{f i t}=2-2.5 \mathrm{~s}^{-1}$.

Directly after the experiment above, the sample was remeasured at $\dot{\gamma}_{0}=9 \mathrm{~s}^{-1}$. Fig. 7.15 iii shows the correlations measured at $10 \mathrm{~s}$ intervals. These correlations are quite similar to the correlations observed at $\dot{\gamma}_{0}=9 \mathrm{~s}^{-1}$ in Fig. 7.15 ii. The central (C), transition $(\mathrm{T})$ and perimeter band $(\mathrm{P})$ are indicated. The absolute variation of the fitted strain rate is largest in the transition band. These observations are difficult to reconcile with a simple application of the lever rule.

\subsection{Discussion}

Backscattering flow-DWS measurements made in the cylindrical-Couette geometry of the wormlike micelles solutions were largely consistent with the behaviour predicted on the basis of the lever rule. The optical flow curve matches the mechanical flow curve well for the three shear banding wormlike micelles solutions examined here. As demonstrated experimentally, shear banding produces a dramatic effect on the DWS correlation functions. It is therefore not surprising that it was possible to readily extract rheological information from these measurements. The comparison between the optical and mechanical flow curves of the shear thinning sample is less satisfactory. Here the correlation deviates less from that expected for uniform shear flow. Systematic errors may be influencing the fit. The Bézier spline fit to the correlations is quite comparable to that produced by the Cross model fit, justifying this novel approach to analysing the data. As illustrated by Fig. 7.6 v-vi the DWS correlation can be acquired rapidly. This temporal resolution may be useful in cases where the flow behaviour shows time dependence as has been observed in a related shear banding wormlike micelles system [36].

The backscattering and transmission flow-DWS measurements made in the coneplate geometry suggest that a high strain rate band expands from the surface of the moving cone and that this shear banding may be accompanied by slip. This interpretation is not entirely unambiguous. The cone-plate measurements of the shear thinning PEO sample clearly indicate that it is possible to extract strain rate in stress controlled conditions. This approach might be useful in situations where it is not possible or practical to control strain rate; for example, in a pressure driven capillary rheometer. The backscattering flow-DWS measurements strongly suggested that the yoghurt sample is shear banding. NMR velocimetry, discussed in [118, demonstrates that this yoghurt does indeed shear band.

The backscattering flow-DWS measurements made in the parallel-plate geometry are difficult to interpret in the sample that shows a stress plateau. Qualitatively, the sample 

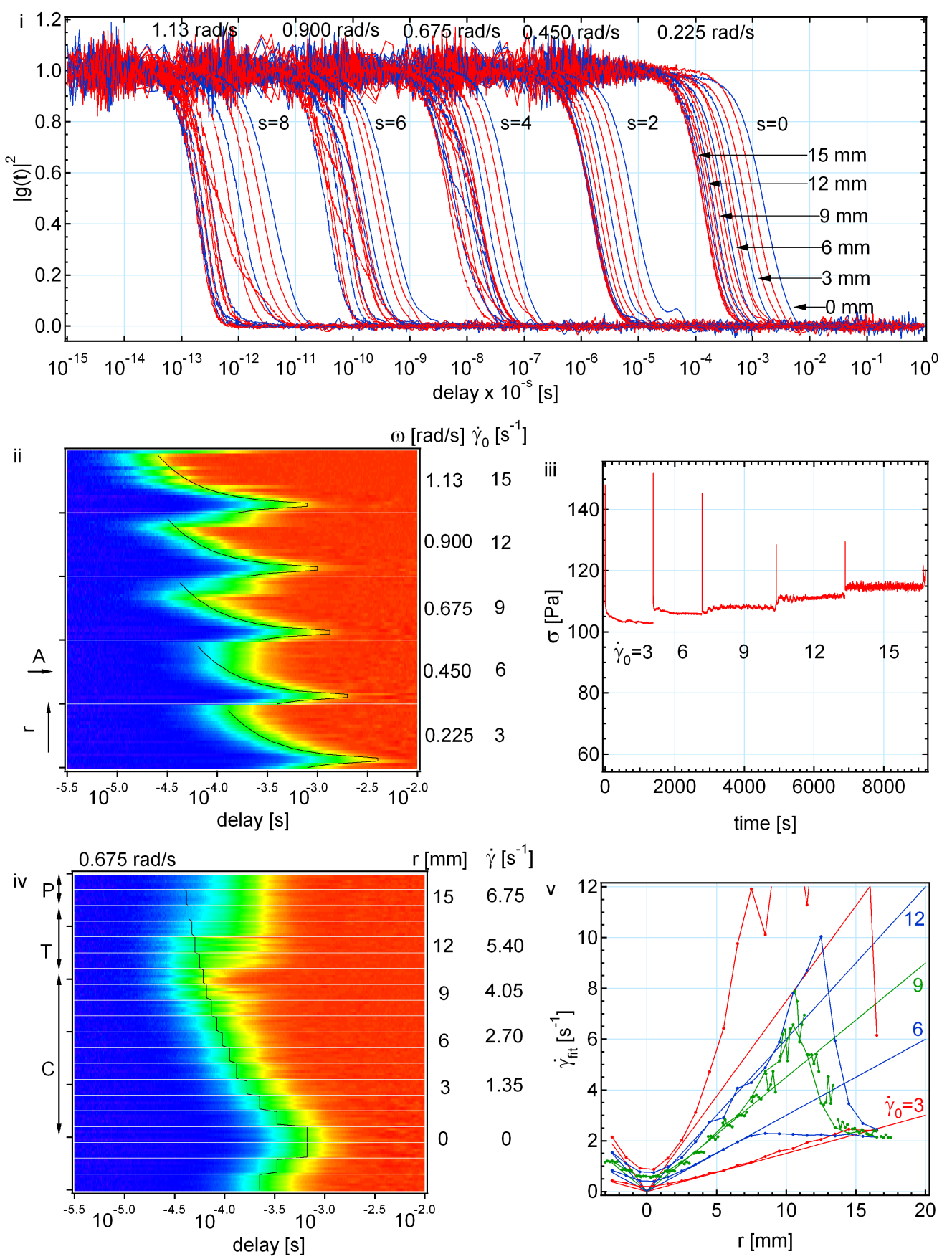

Figure 7.15: i) Backscattering flow-DWS measurements from the $\phi=8$ wt. $\%$ RHB sample with radial position and angular velocity in the parallel-plate geometry. ii) Data in i mapped to a surface. The black line indicates the delay required for the $\left|g_{1}(t)\right|^{2}=0.5$ if $\dot{\gamma}=v / d$. From point A the correlations show essentially no change with increasing radius at $\dot{\gamma}_{0}=6 \mathrm{~s}^{-1}$. iii) Measured stress during the flow-DWS experiment illustrating the protocol used. iv) Repeated measurement at $\dot{\gamma}_{0}=9 \mathrm{~s}^{-1}$ with a higher time resolution. Qualitatively, the measured correlations partition into a centre $(\mathrm{C})$, transition $(\mathrm{T})$ or perimeter $(\mathrm{P})$ band. v) Fitted strain rate from a fit to the uniform shear flow model. 
appears to divide into three radial bands: a central band $(\mathrm{C})$ where the local strain rate is directly proportional to the apparent strain rate, a transition band $(\mathrm{T})$ where correlations resemble those seen for shear banding wormlike micelles and a perimeter band $(\mathrm{P})$ with a constant strain rate (see Fig. $7.15 \mathrm{v}$ ). This behaviour is difficult to reconcile with a simple application of the lever rule. Clearly, additional measurements in other optical configurations would have been helpful here.

The flow-DWS measurements here indicated that the $l^{*}$ value for the solutions studied was $l^{*} \approx 0.18 \mathrm{~mm}$. This value is approximately $30 \%$ smaller than the theoretical value of $l^{*}=0.25 \mathrm{~mm}$ given in [87] for a $1 \%$ suspension of polystyrene sphere in water at $k a=2 \pi /(633 / 1.33) \times 500 \times 10^{-9} \approx 6$. This may reflect an error in the estimate of the concentration of spheres present in solution after washing.

If the probes are monodisperse then it is possible, in principle, to study the relationship between diffusion and strain rate of the suspended particles in these fluids. Unfortunately, the laser used here was quite noisy, producing artefacts in the correlations at longer delay times which prevented the study of this relationship in wormlike micelles in the backscattering geometry.

It was straightforward to simulate the propagators $G_{n}$ and $\rho_{n}$ using a simple particle diffusion model. While not immediately useful here, simulation could be used to model these propagators in more complex geometries, such those found in industrial applications, which are not easily amenable to analytic analysis. One interesting example which does not appear to have been described in the literature is a focused beam geometry in the backscattering configuration with a variable separation between the source and detection points. Increasing the separation between the source and detection points (by translating the collection optics, for example) will shift the path length distributions to longer paths that probe deeper into the material.

The analysis developed by Bicout and co-workers could be applied to any kind of relative motion. Dimensional analysis suggests that dephasing for a one-dimensional gradient in diffusion is of the form

$$
\left\langle\Delta \phi_{n}^{2}(t)\right\rangle=2 k^{2} n \int D(x) t \rho_{n}\left(x_{o}, x_{i}, x, l^{*}\right) d x .
$$

A gradient in the diffusion constant might result from a difference in viscosity (due to a temperature gradient, for example) across a turbid medium. 


\section{Chapter 8}

\section{Investigating the correlation between strain rate and optical anisotropy in wormlike micelles solutions}

\subsection{Introduction}

In Chapter 7 evidence for shear banding in wormlike micelles was presented using DWS. While the temporal resolution of this rheo-optic technique is 'good', its spatial resolution is 'poor'. Spatial resolution is traded for temporal resolution in this chapter where a related rheo-optical technique, homodyne PCS, is used in conjunction with ellipsometry to examine more closely the spatial partitioning that occurs under shear in wormlike micelles solutions.

As noted in Chapter 3, the flow behaviour of wormlike micelles solutions is generally studied using one of two approaches. In the first approach, the fluid's velocity is measured as a function of position. Shear rate can be determined from the velocity profile by differentiation. Velocity has been studied extensively in the Authors own lab using nuclear magnetic resonance (NMR) [26] 31] [32] 33] 34] 35] [95] 36]. Heterodyne photo-correlation spectroscopy (PCS) [27, ultrasonic velocimetry [120] and particle imaging velocimetry (PIV) [121] have also been used to study the velocity of sheared wormlike micelles solutions on the stress plateau. Generally speaking, these velocimetry measurements do confirm that shear banding occurs when $\dot{\gamma}>\dot{\gamma}_{c}$. In the second approach, the orientational ordering of the fluid is determined. Elongated particles experience a viscous torque that tends to rotate them about the vorticity axis. This is countered by an elastic torque which arrests rotation leaving the particles with a net alignment. In this approach, the strain rate is inferred from the degree of alignment. Orientational ordering has been examined using flow birefringence [122, 24] [123] [25] [40] [124] [125] [126] [127] 63] [42] 
[128] [129] [43] [44] 66] [104] 64], small angle light scattering (SALS) [123] [130] [42] [131] 62 [75, small angle neutron scattering (SANS) 38] [132] [133] [25] [134 [66] and NMR [35] [103 [36. Flow birefringence and NMR have been used to spatially resolve ordering across a sheared solution. Optical measurements clearly demonstrate a partitioning into bands of differing optical anisotropy; these bands have been widely interpreted as shear bands. At higher concentrations, a solution of wormlike micelles will spontaneously form an orientated nematic phase. This has led some authors to speculate that shear banding is caused by a shear induced non-equilibrium phase transition from a high viscosity isotropic state to a low viscosity highly ordered nematic state; an effect seen in some lyotropic liquid crystals. SANS spectra have been decomposed into isotropic and nematic components and are cited as evidence for isotropic to nematic (I-N) phase transition in a number of cases. SALS spectra indicate increasing order with strain rate.

NMR is unique among the techniques described above in that it can be used to measure velocity and ordering simultaneously. In [35] and [135] Fisher and Callaghan used this dual capability to examine ordering and velocity in a concentrated cetylammonium bromide $(\mathrm{CTAB})$ in a $\mathrm{D}_{2} \mathrm{O}$ wormlike micelles solution $\left(\phi_{\mathrm{CTAB}}=21 \%\right)$ at temperatures near the equilibrium isotropic to nematic (I-N) phase transition for this system. This system had been studied before using both SANS and flow birefringence. Because of the proximity to the equilibrium I-N transition, this system was expected to be a good candidate for a non-equilibrium phase transition. However, partitioning into birefringence bands observed in [35] seemed inconsistent with that predicted according to the lever rule. Significantly, the NMR study demonstrated that for this system at least, the correlation between strain rate and ordering was poor.

More recently, Lerouge, Decruppe and Olmsted [44] revisited the equi-molar semidilute $\mathrm{CTAB}$ and $\mathrm{KBr}([\mathrm{CTAB}]=[\mathrm{KBr}]=0.3 \mathrm{M})$ in water wormlike micelles system initially studied by Decruppe and co-workers [40] using flow birefringence. The more recent work indicated that the relationship between strain rate and optical anisotropy was more complicated than initially thought.

In this chapter, the relationship between optical anisotropy and strain rate is investigated in sheared wormlike micelles solutions using both homodyne photo-correlation spectroscopy and ellipsometry. As will be demonstrated, the method allows the determination of both the local strain rate and optical anisotropy simultaneously with a spatial resolution of about $100 \mu \mathrm{m}$.

\subsection{Experimental}

\subsubsection{Set-up}

Optical measurements were made using a homemade rheo-optical cylindrical-Couette flow cell. The cell used is illustrated in Fig. 8.1 i. The dimensions of the cell were based on those described in [43]. The radius of the outer wall of the rotating bob was $r_{i}=22 \mathrm{~mm}$ 
i
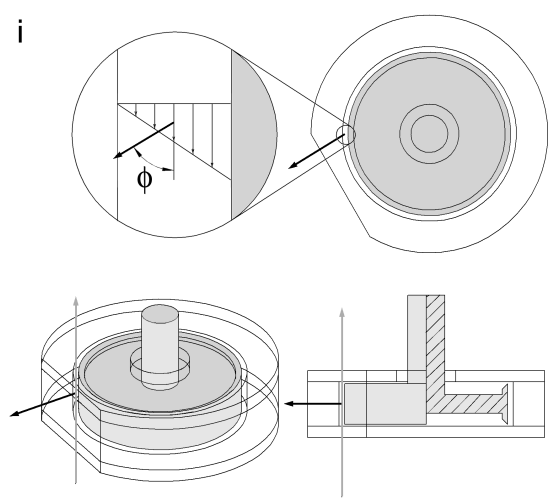

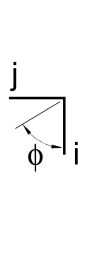

$\theta$.

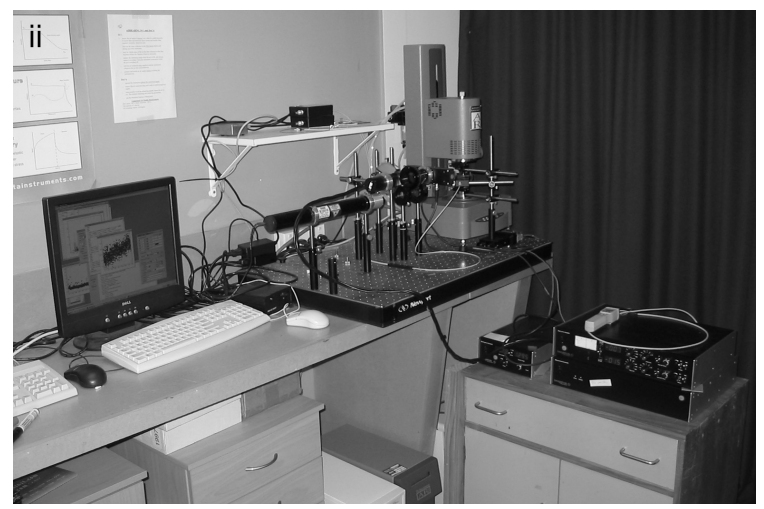

Figure 8.1: i) Top, side and oblique view of the homemade rheo-optic cylindrical-Couette cell with a detailed view of gap. The dark arrow indicates the direction of the collection optics for the homodyne PCS measurements. ii) Photograph of the experimental set-up.

and the inner wall of the stationary cup was $r_{o}=23.5 \mathrm{~mm}$ for a gap $d=1.5 \mathrm{~mm}$. The height $h$ of the bob was $h=10 \mathrm{~mm}$. The bob was made of aluminium and anodised black to prevent reflections. The side walls, base and cap of the geometry were made of transparent Perspex. Light was introduced into the gap through the base from beneath. The transmitted component was collected from above, through the cap and a scattered component collected through the side wall in the $\hat{\mathbf{i}}-\hat{\mathbf{j}}(\mathbf{v}-\nabla \mathbf{v})$ plane at an angle $\phi$ with respect to the flow direction of the sheared fluid. A segment of the cylinder was cut away and the normal of the plane surface was directed at the collection optics in order to reduce the effect of refraction at the Perspex/air interface on the scattering volume. This plane surface was masked with tape to ensure that only light scattered from a mid-height position in the gap was collected.

A schematic of the optical set-up is shown in Fig. 8.2. Light from a $15 \mathrm{~mW}$, $\lambda=633 \mathrm{~nm}$, HeNe laser (Melles Griot) is expanded and directed through a $f=300 \mathrm{~mm}$ plano-convex condenser, a sheet polaroid polariser mounted in a manual goniometer, and onto a tilt-stage (Edmund Optics) mounted protected gold right angle mirror (Edmund Optics) which reflects light into the homemade optical cylindrical-Couette flow cell. The transmitted component is reflected from a second, identical, tilt-stage mounted mirror through a $0.5 \mathrm{~mm}$ pinhole, a photoelastic modulator (Beaglehole Instruments Ltd., BIL) a second identical sheet polaroid analyser mounted in a manual goniometer, a variable neutral density filter (Edmund Optics) and into a silicon detector (BIL). The amplitude of the AC components of the Si signal, coherent with photoelastic modulation, are measured on a dual lock-in amplifier (BIL) and acquired along with a DC component at the measurement controller (BIL). These AC and DC components are combined at the host PC to give a measurement of the fluid's ellipsometry. Scattered light is collected using a gradient index (GRIN) objective carefully aligned on the beam. The scattered light is directed out through a single mode fibre optic beam splitter (FONT) to two photon counting 


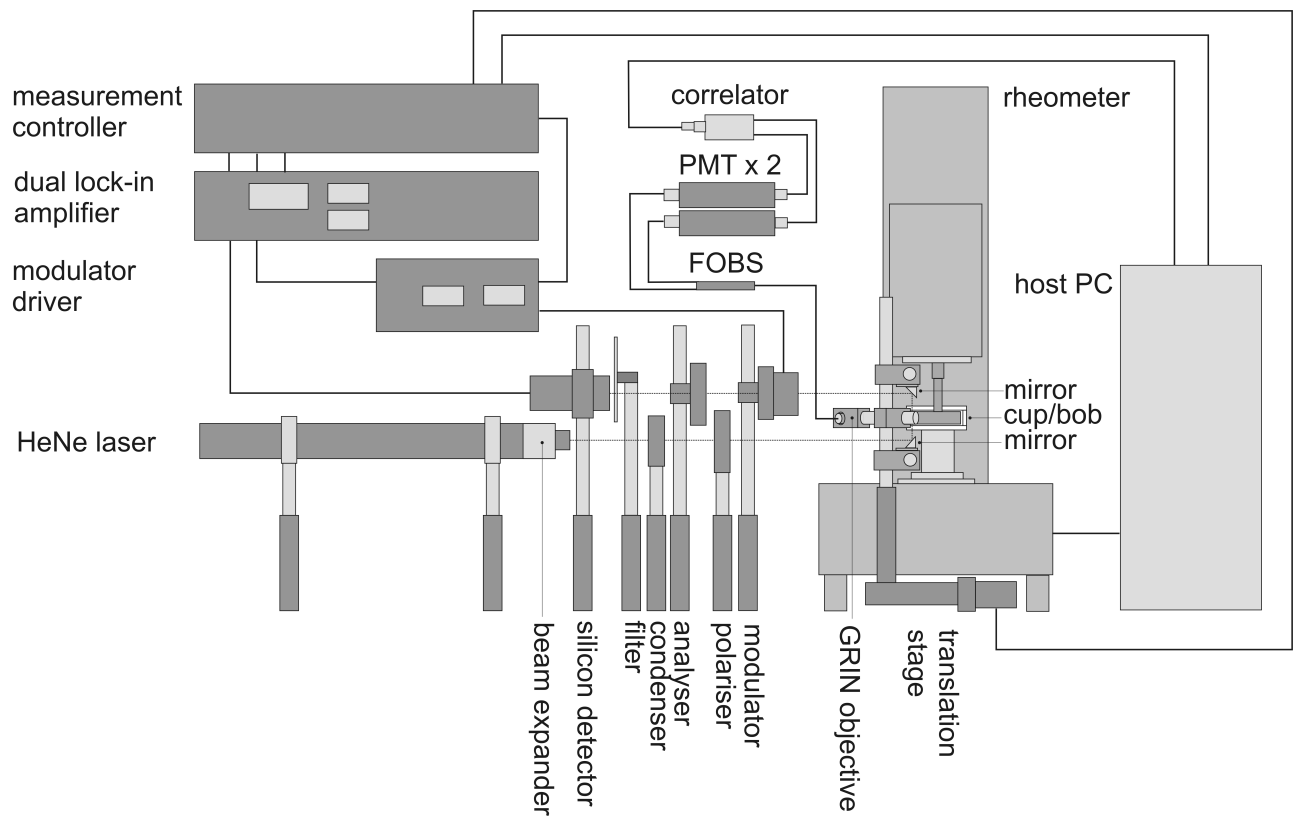

Figure 8.2: Schematic of the experimental homodyne-PCS/ellipsometry set-up.

photomultipliers (correlator.com). The photomultipliers' output is cross-correlated on a Flex02OEM-12C hardware correlator (correlator.com). Once acquired, the raw correlation is communicated to the host PC. The mirrors and GRIN assembly are mounted off posts mounted on a computer controlled motorised translation stage. By translating the stage, the beam can be scanned through the gap; because the GRIN objective is also mounted on the translation stage, focusing optics/beam separation are maintained.

Additional optical measurements were made using a Beaglehole Instruments 'Picometer' - a spectroscopic variable angle birefringence modulation ellipsometer and a Malvern '4700 Autosizer' - a variable angle photo-correlation spectrometer equipped with a $500 \mathrm{~mW}, \lambda=532 \mathrm{~nm}, \mathrm{Nd}: Y A G$ diode laser. All rheo-optical measurements were made at $22.1 \pm 0.3^{\circ} \mathrm{C}$ in a temperature stabilised room. Mechanical measurements were made on a TA AR2000 stress controlled rheometer operated in strain rate controlled mode.

\subsubsection{Samples}

RHB wormlike micelles solutions at weight fractions of $\phi_{\mathrm{CPCl}+\mathrm{NaSal}}=3,4,5,6,7,8,9$ and 10 wt.\% were examined. The rheology of the samples used were checked using an acrylic $40 \mathrm{~mm}$ diameter $1 \mathrm{deg}$ cone and plate at $22{ }^{\circ} \mathrm{C}$. The $\phi=7 \mathrm{wt} . \%$ sample was examined in detail over intervals of $600 \mathrm{~s}$ at various strain rates. Measurements of the stress are shown in Fig. 8.3 ii. At strain rates between $\dot{\gamma}=1.00$ and $35.5 \mathrm{~s}^{-1}$ small fluctuations in the stress were observed - these are coherent with the rotation of the cone. At $\dot{\gamma}=39.8$ 

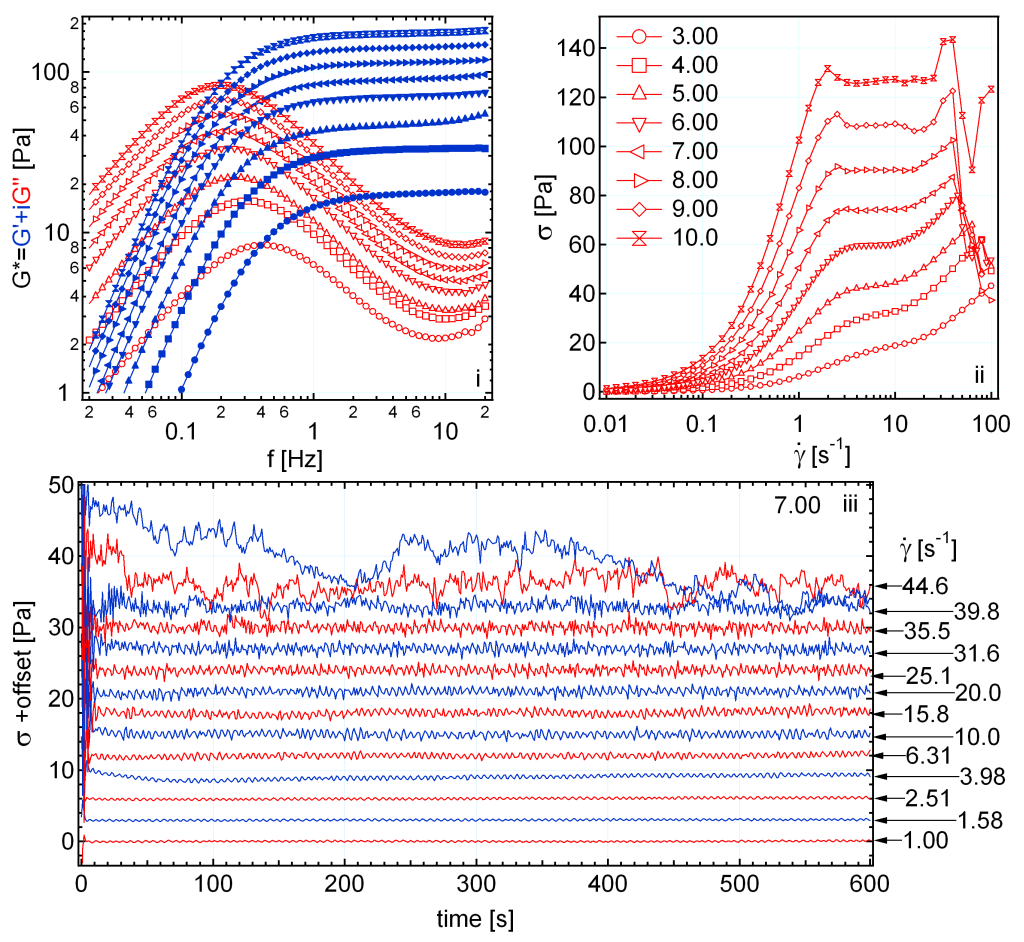

Figure 8.3: i) Linear and ii) nonlinear rheology of the RHB wormlike micelles solution studied. iii) Time resolved stress measurements during a stepped strain rate test for the $\phi=7$ wt.\% RHB sample.

$\mathrm{s}^{-1}$, incoherent fluctuations develop, possibly signaling the development of rheo-chaos in the sample.

\subsubsection{Homodyne photo-correlation spectroscopy}

Strain rate was measured using homodyne PCS. In a conventional homodyne PCS experiment, the time variation of intensity is measured via an intensity autocorrelation $\langle I(t) I(0)\rangle /\langle I\rangle^{2}$. This measured intensity correlation can be related to the field correlation $g_{1}(t)$ by

$$
\langle I(t) I(0)\rangle /\langle I\rangle^{2}=1+\beta\left|g_{1}(t)\right|^{2} .
$$

In order to understand how strain rate was determined from this correlation, it is instructive to first consider the effect of diffusion. As discussed in Chapter 4, in the absence of flow, the PCS field correlation function for a monodisperse suspension (in a Newtonian dispersant) is expected to follow

$$
\left|g_{1}(t)\right|=\exp \left[-q^{2} D t\right]
$$


For a bidisperse suspension, the field correlation is a weighted sum

$$
\left|g_{1}(t)\right|=A \exp \left[-q^{2} D_{f} t\right]+(1-A) \exp \left[-q^{2} D_{s} t\right] .
$$

Here $D_{f}$ is the diffusion constant of the 'faster' component and $D_{s}$ is the diffusion constant of the 'slower' component. The weighting factor $A$ depends on relative concentration and scattering cross-sections. Measured correlations for a monodisperse $(r=500 \mathrm{~nm})$ and bidisperse $\left(r_{f}=500 \mathrm{~nm}, r_{s}=45 \mathrm{~nm}\right)$ latex sphere suspension in a water/glycerol solution are compared to model calculations in Fig. $8.4 \mathrm{i}$-ii. Measurements were made on a Malvern Autosizer and were log-spaced in $q^{2}$. The viscosity of the dispersant (which determines the diffusion constants $D, D_{s}$ and $D_{f}$ through the Stoke-Einstein relation) and weighting factor $A$ were determined by fitting the data to Eqn. 8.2 and 8.3 . The fit shows good agreement with the data.

Fig. 8.4 iii-iv shows correlations for a semi-dilute 3 wt.\% RHB wormlike micelles solution and a semi-dilute 1 wt.\% 5-6 M polyacrylamide (PAC) in water solution. Like the bidisperse suspension, the relaxation is roughly biexponential as was predicted for semi-dilute solutions in Chapter 4. For the RHB sample, the fast mode shows $q$ dependence whereas the slow mode does not, and the relative amplitude of the two modes is approximately constant. This is the behaviour predicted for a viscoelastic fluid dominated by a single relaxation mode [92. The behaviour of the PAC solution is more complicated. Like the RHB sample, the correlation is approximately biexponential. Unlike the RHB sample, the relative amplitude of the fast and slow mode is not constant and the slow mode shows $q$ dependence. Linear rheometry indicated that this fluid is not Maxwell-like. The deviation from single mode behaviour may explain why the relative amplitude and characteristic time of the slow mode shows $q$ dependence. A number of the RHA and RHB samples were measured on the Malvern autosizer at a fixed angle of $\theta=30 \mathrm{deg}$. The correlations shown in Fig. $8.5 \mathrm{i}$ and iii were fitted to a biexponential relaxation of the form $g_{1}(t)=A \exp \left[-t / \tau_{f}\right]+[1-A] \exp \left[-t / \tau_{s}\right]$. Fitted $\tau_{s}$ and $\tau_{f}$ are reported in Fig. 8.5 ii and iv. The characteristic time of the slow mode $\tau_{s}$ is compared to the Maxwell time of the sample $\tau_{M}$. $\tau_{s}$ is correlated with $\tau_{M}$.

In Chapter 4, only a passing reference was made to the dimensions of the scattering volume. The scattering volume can influence the measured correlation in PCS. For a monodisperse suspension in a Newtonian fluid undergoing diffusion and shear flow ([136] or [12])

$$
\left|g_{1}(t)\right|=\exp \left[-q^{2} D t\right] \int_{V} I(\mathbf{r}) \exp [-i \mathbf{q} \cdot \underline{\underline{G}} \cdot \mathbf{r} t] d \mathbf{r},
$$

where $\underline{\underline{G}}=\nabla \mathbf{v}$ is the velocity gradient tensor. In the configuration outlined in the last section $\mathbf{k}_{i}=-k \hat{\mathbf{k}}$ and $\mathbf{k}_{f}=k \sin \phi \hat{\mathbf{i}}+k \cos \phi \hat{\mathbf{j}}$ and

$$
\underline{\underline{G}}=\left[\begin{array}{ccc}
0 & \dot{\gamma} & 0 \\
0 & 0 & 0 \\
0 & 0 & 0
\end{array}\right]
$$



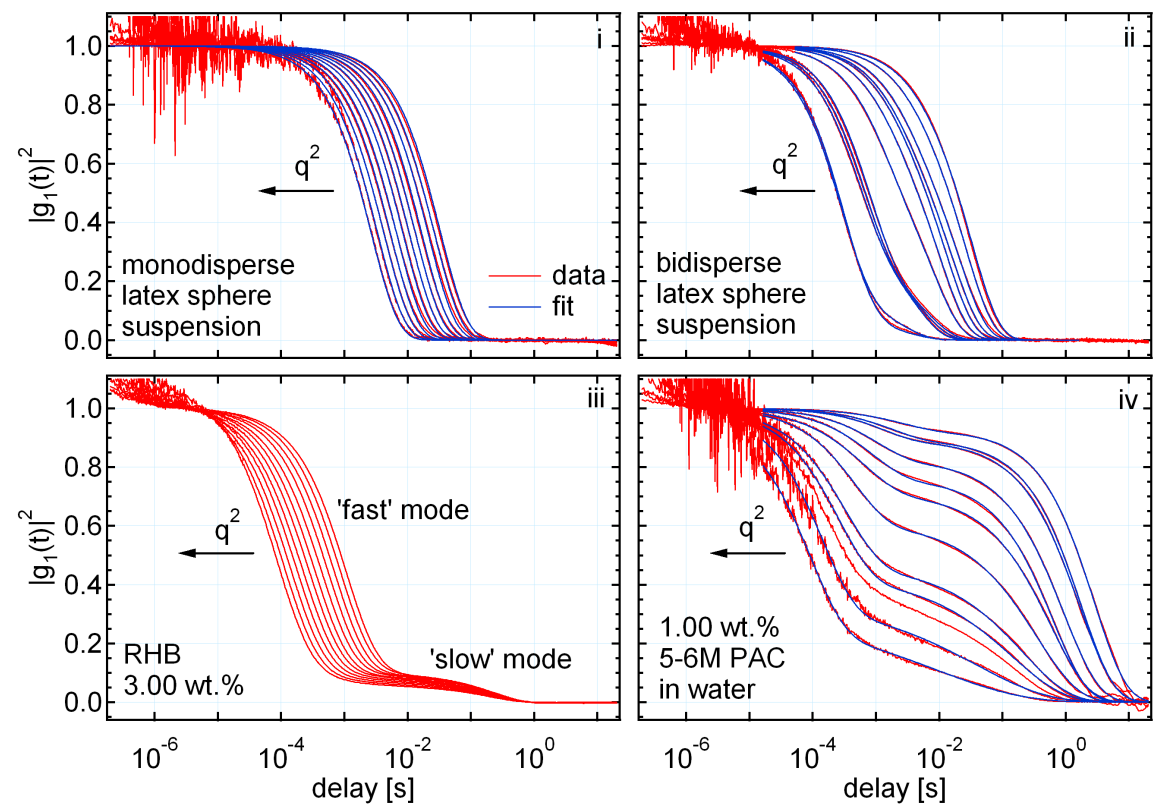

Figure 8.4: Equilibrium correlations measured for a i) monodisperse and ii) bidisperse latex sphere suspension and a semi-dilute iii) wormlike micelles solution and iv) polyacrylamide solution as a function scattering angle. 

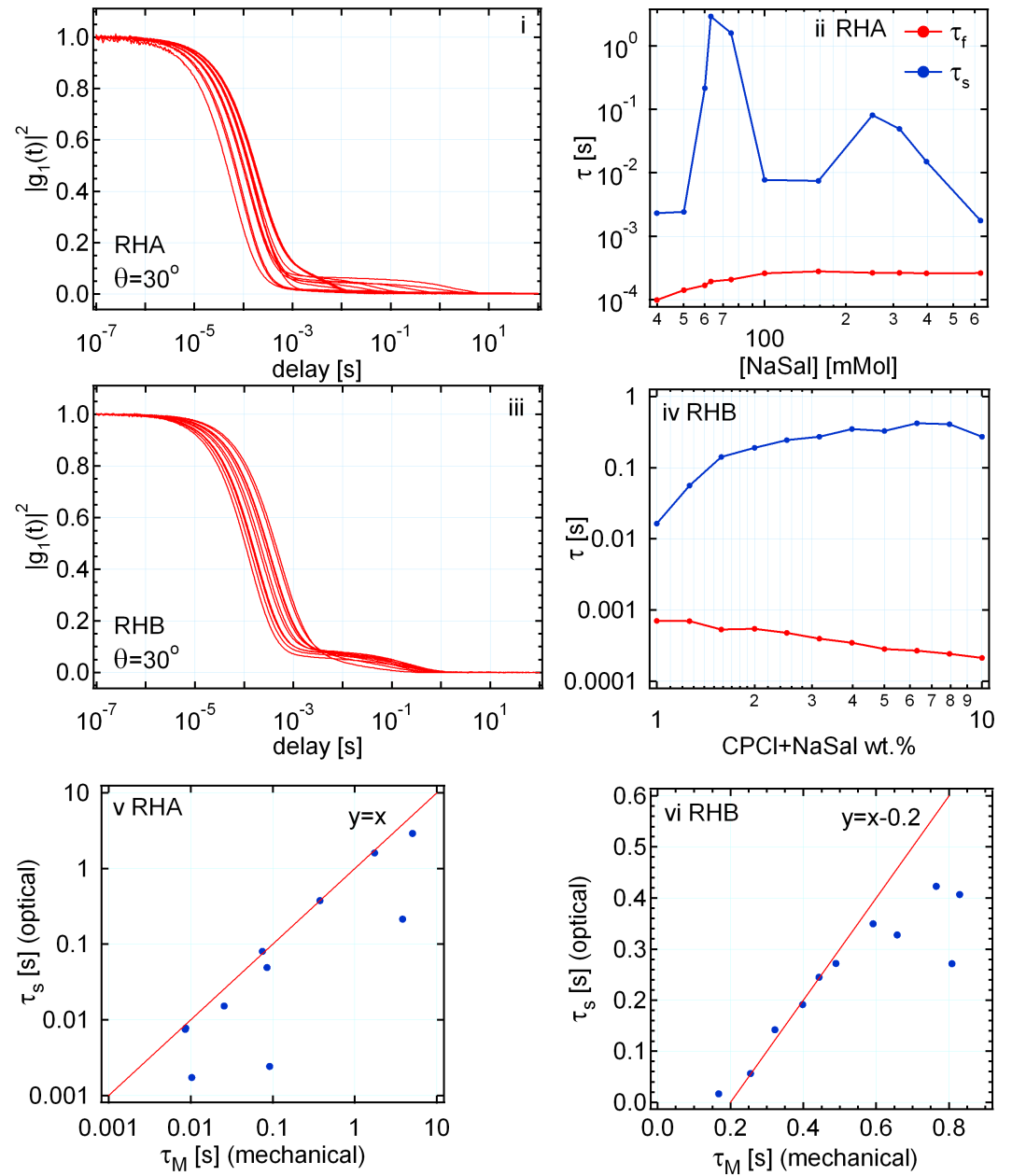

Figure 8.5: i and iii) Equilibrium correlations measured at a fixed scattering angle for selected RHA and RHB wormlike micelles solutions with ii and iv) fitted characteristic relaxation times. v-vi) A comparison between the optically measured characteristic time tau $_{s}$ and the mechanically measured characteristic time $\tau_{M}$. 
so that $\mathbf{q} \cdot \underline{\underline{G}} \cdot \mathbf{r}=q \dot{\gamma} y \cos \phi$ where $q=2 k \sin [\theta / 2]$. The polar angle $\theta$ is fixed at $\theta=90$ deg in the rheo-optical configuration here. Assuming a Gaussian scattering volume $I(\mathbf{r})=$ $\exp \left[-\left(x^{2}+y^{2}+z^{2}\right) / L^{2}\right]$

$$
\left|g_{1}(t)\right|=\exp \left[-q^{2} D t\right] \exp \left[-(q L \dot{\gamma}(r) \cos \phi t)^{2} / 4\right] .
$$

$\dot{\gamma}(r)$ is the local strain rate in the scattering volume. Note that if shear flow is absent Eqn. 8.6 reduces to Eqn. 4.61. Only interference effects between the field reradiated by the scattering centres are considered in this analysis. The temporal statistics can also be influenced by so called 'transit' effects. The scattered intensity depends on the number of scattering centres in the scattering volume. This number will fluctuate as the scattering centres pass through or 'transit' the scattering volume introducing a fluctuation into the scattered intensity with a characteristic time that depends on the type of motion and dimensions of the scattering volume. With transit effects $\left|g_{1}(t)\right|$ is given by:

$$
\left|g_{1}(t)\right|=\underbrace{\exp \left[-q^{2} D t\right] \exp \left[-(q L \dot{\gamma}(r) \cos \phi t)^{2} / 4\right]}_{\text {interference }} \underbrace{\exp \left[-(v(r) t / L)^{2} / 2\right] \exp \left[-\left(D t / L^{2}\right)\right]}_{\text {transit }},
$$

where $q=2 k \sin [\theta / 2]=k \sqrt{2}$ and $v(r)$ is the local velocity (this result was verified through extensive simulation).

The characteristic time of the last exponent in Eqn. 8.7 is generally very large and will be ignored here. The velocity dependence can be neglected if $\tau_{v}=[v / L \sqrt{2}]^{-1}$ is significantly larger than $\tau_{\dot{\gamma}}=\left[\frac{1}{2} q L \dot{\gamma}_{l} \cos \phi\right]^{-1}$ and $\tau_{D}=\left[q^{2} D\right]^{-1}$. Fig. 8.6 i shows the integrated intensity profile of the laser beam at the beam waist, measured by translating at knifeedge (razor) through the beam. From a fit to an error function $A\left(\frac{1}{2}+\operatorname{erf}\left[\left(x-x_{0}\right) / L\right]\right)$ it is estimated that the radius of the beam is $L=45 \mu \mathrm{m}$. Assuming that $v=\dot{\gamma} d$ and that $d=1.5 \times 10^{-3} \mathrm{~m}$, that $\phi=60 \mathrm{deg}$ and that $q=1.87 \times 10^{7} \mathrm{~m}^{-1}$ it is found that $\tau_{v} / \tau_{\dot{\gamma}}=8.9$. This corresponds to the extreme situation where the fluid is profiled at the moving wall. In practice, only the central region of the gap from $(2 / 15-13 / 15) \times d$ can be accessed. Over this range of positions $\tau_{v} / \tau_{\dot{\gamma}}=10-67$. If the strain rate of a Newtonian fluid is estimated without considering the contribution of velocity, the estimated strain rate will exceed the true strain rate by a factor of only 1.097 to 1.014 . Shear thinning and shear banding are expected to increase the local strain rate near the moving surface (while decreasing the strain rate at the stationary surface) reducing the (average) error introduced by neglecting transit times - the contribution of transit times at $\phi=60 \mathrm{deg}$ will be neglected.

At $\phi=90 \mathrm{deg}$ the correlation is insensitive to strain rate. In principle it is possible to velocity profile a fluid in this configuration using transit times. This is demonstrated in Fig. 8.6 ii. Here a suspension of diameter $r=88 \mathrm{~nm}$ latex spheres in glycerol was examined at $\phi=90 \mathrm{deg}$ by rotating the geometry and repositioning the collection optics. According to Eqn. 8.7 the correlation half time $\tau_{v, 1 / 2},\left|g_{1}\left(\tau_{v, 1 / 2}\right)\right|^{2}=0.5$ is inversely proportional to velocity. Measured $\tau_{v, 1 / 2}^{-1}$ is plotted as a function of position for a series 

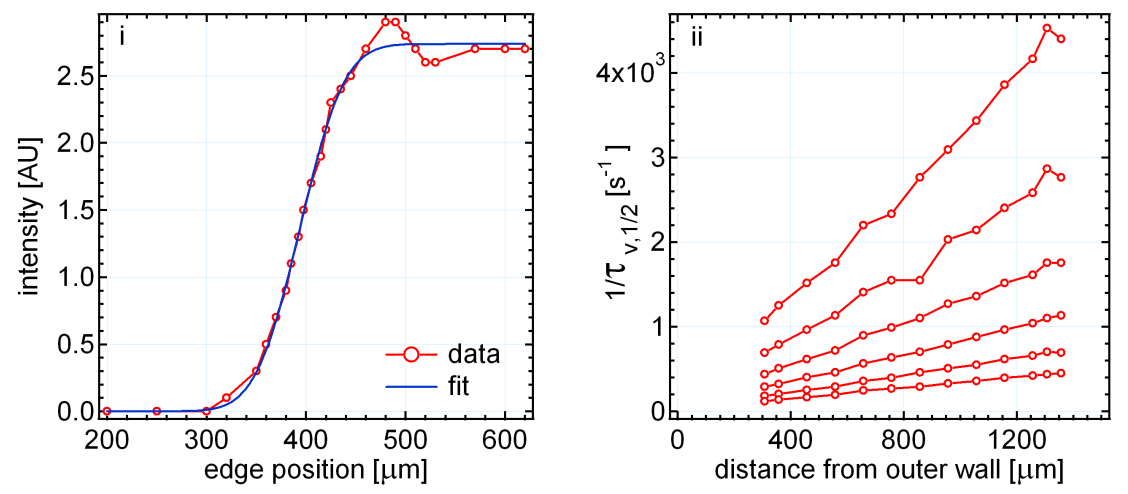

Figure 8.6: i) Measured integrated intensity profile with a fit at the beam waist. ii) Dependence of the reciprocal of the correlation half time on the position in the gap for the strain rate indicated and at a scattering angle $\phi=90 \mathrm{deg}$. The sample was a monodisperse suspension of latex spheres in glycerol. These measurements indicate that transit time dependence can be used to infer velocity.

of log-spaced strain rates. $\tau_{v, 1 / 2}^{-1}$ varies linearly across the gap and is proportional to the apparent strain rate as is expected for a Newtonian fluid.

In order to test the set-up a : 1) monodisperse suspension of $r=500 \mathrm{~nm}$ latex spheres in water; 2) bidisperse suspension of $r=500 \mathrm{~nm}$ and $r=40 \mathrm{~nm}$ latex spheres in water; 3) 1 wt.\% solution of 5-6 M polyacrylamide (Polyscience) in water and 4) $\phi=3$ and 7 wt.\% RHB wormlike micelles solution were examined in the optical cylindrical-Couette cell under equilibrium conditions (zero shear) and under conditions of flow. Measurements were made mid-gap and at $\phi=60 \mathrm{deg}$ with variable apparent strain rate.

Measurements of the monodisperse and bidisperse suspension are shown in Fig. 8.7 i-ii. For the monodisperse suspension, the measured correlation is similar to the calculated correlation and scales correctly with strain rate but shows a different time dependence; the exponent of $\dot{\gamma} t$ differing from the value of 2 calculated on the assumption of a Gaussian scattering volume. This behaviour was generally observed for all Newtonian samples examined - it was assumed that this was due to the scattering volume being non-Gaussian. It was found experimentally that the correlation was well described by the function

$$
\left|g_{1}(t)\right|=\exp \left[-q^{2} D t\right] \exp \left[-(\alpha \dot{\gamma}(r) t)^{p}\right],
$$

where $D=k_{B} T / 6 \pi \eta r=4.3 \times 10^{-13} \mathrm{~m}^{2} / \mathrm{s}, p=1.5$ and $\alpha=130$ is a dimensionless constant of proportionality which might be expected to show $q L \cos \phi$ dependence. Assuming the same time dependence as was observed above, it would be expected that the bidisperse correlation would be described by

$$
\left|g_{1}(t)\right|=\left(A \exp \left[-q^{2} D_{f} t\right]+[1-A] \exp \left[-q^{2} D_{s} t\right]\right) \times \exp \left[-(\alpha \dot{\gamma}(r) t)^{p}\right] .
$$

$\left|g_{1}(t)\right|^{2}$ is calculated for $D_{f}=5.4 \times 10^{-12} \mathrm{~m}^{2} / \mathrm{s}, D_{s}=4.3 \times 10^{-13} \mathrm{~m}^{2} / \mathrm{s}, A=0.72, p=1.5$ 

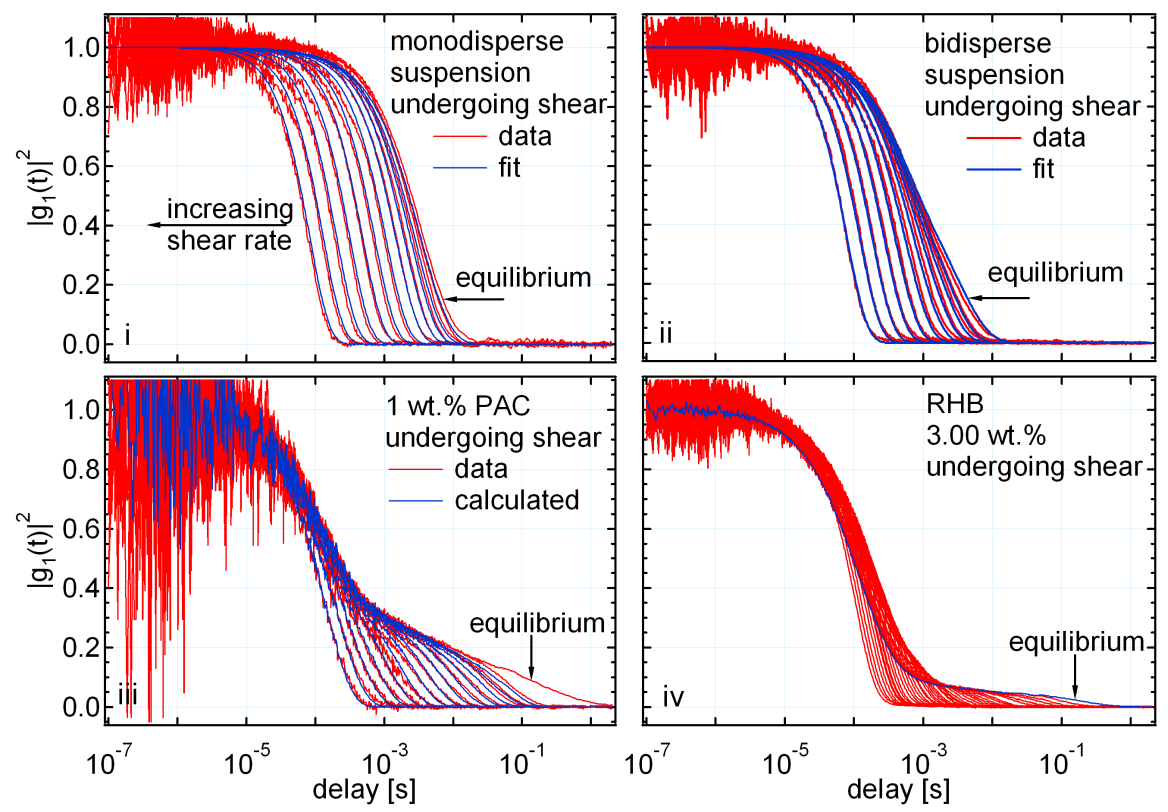

Figure 8.7: i and ii) Measured correlations with fit for the monodisperse and bidisperse latex sphere suspensions in water in flow conditions. iii) Measured and calculated correlations for a 1 wt.\% polyacrylamide in water. Note that the polyacrylamide data have been baseline corrected. iv) Measured correlations for a $\phi=3 \mathrm{wt} . \%$ RHB wormlike micelles solution. 
and $\alpha=130$ for the bidisperse suspension shown in Fig. 8.7 ii. The experimental and model data show reasonable agreement.

Measured correlations for the $1 \mathrm{wt} . \%$ solution of 5-6 M polyacrylamide in water are reported in Fig. 8.7 iii. Note that these measurements have been baseline corrected for the effects of dust in solution on the correlations. Assuming that the equilibrium relaxation modes are unaffected by shear and that this system exhibits the same time dependence observed for the Newtonian fluids above, it would be expected that the correlations could be described by

$$
\left|g_{1}(t)\right|=\left|g_{1,0}(t)\right| \exp \left[-(\alpha \dot{\gamma}(r) t)^{p}\right],
$$

where $\left|g_{1,0}(t)\right|$ is the field correlation function measured in the absence of shear. Correlations calculated according to Eqn. 8.10 and assuming $p=1.5$ and that $\alpha=130$ are also shown in Fig. 8.7 iii. Again the experimental and analytic data show reasonable agreement.

Correlations measured for the $\phi=3$ and 7 wt.\% RHB solutions are shown in Fig. 8.8. At equilibrium, the correlations exhibit behaviour similar to that observed for the polyacrylamide solution. It might therefore be expected that under shear flow conditions the correlation would behave according to Eqn. 8.10 (note that the diffusion of the surfactant/counterions is implicit in the $\left|g_{1,0}(t)\right|$ term). The wormlike micelles solutions were observed to become more turbid on shearing - this behaviour was not observed in the polyacrylamide solution. The model described by Eqn. 8.10 does not explicitly account for these additional scattering centres. If these structures were sufficiently large, their diffusion could be neglected. Adapting the model described by Eqn. 8.10 to include these new scattering centres and neglecting their diffusion gives

$$
\left|g_{1}(t)\right|=\left([1-B]\left|g_{1,0}(t)\right|+B\right) \times \exp \left[-(\alpha \dot{\gamma}(r) t)^{p}\right] .
$$

$B(\dot{\gamma}(r))$ describes the contribution of the large shear induced structures to the correlation. A fit to the data for a subset of measurements for the $\phi=3$ wt. $\%$ and 7 wt. $\%$ samples undergoing shear to determine the parameter $B$ and $\dot{\gamma}(r)$ is illustrated in Fig. $8.9 \mathrm{i}$ and ii along with fit residuals in 8.9 iii and iv. In both cases $\left|g_{1,0}(t)\right|$ was determined from an initial measurement in the equilibrium conditions and values of $\alpha=130$ and $p=1.5$ were assumed. The two parameter per correlation fit accurately captures the behaviour of the measured correlations. Fitted $\dot{\gamma}(r)$ for the $\phi=3$ wt.\% sample are shown in Fig. 8.9 i. It is found that $\dot{\gamma}(r)$ is directly proportional to $\dot{\gamma}$ - as will be seen in Fig. 8.15 this behavior is expected for this 'weakly' shear thinning fluid. The polyacrylamide data were also fitted to Eqn. 8.11 with $\left|g_{1,0}(t)\right|$ determined from the initial measurements made in the equilibrium conditions and with values of $\alpha=130, p=1.5$ and $B=0$ (no shear induced turbidity) assumed. Fitted $\dot{\gamma}(r)$ is also shown in Fig. 8.9 i. Again it is found that $\dot{\gamma}(r)$ is directly proportional to $\dot{\gamma}$. In addition to measuring the variation of the intensity with time, the DC or average scattered intensity at each strain rate was measured. Fig. 8.9 ii shows the normalised average excess scattered intensity $\left[I / I_{0}-1\right]$ where the data has been normalised with respect to the average scattered intensity in the no shear condition. 

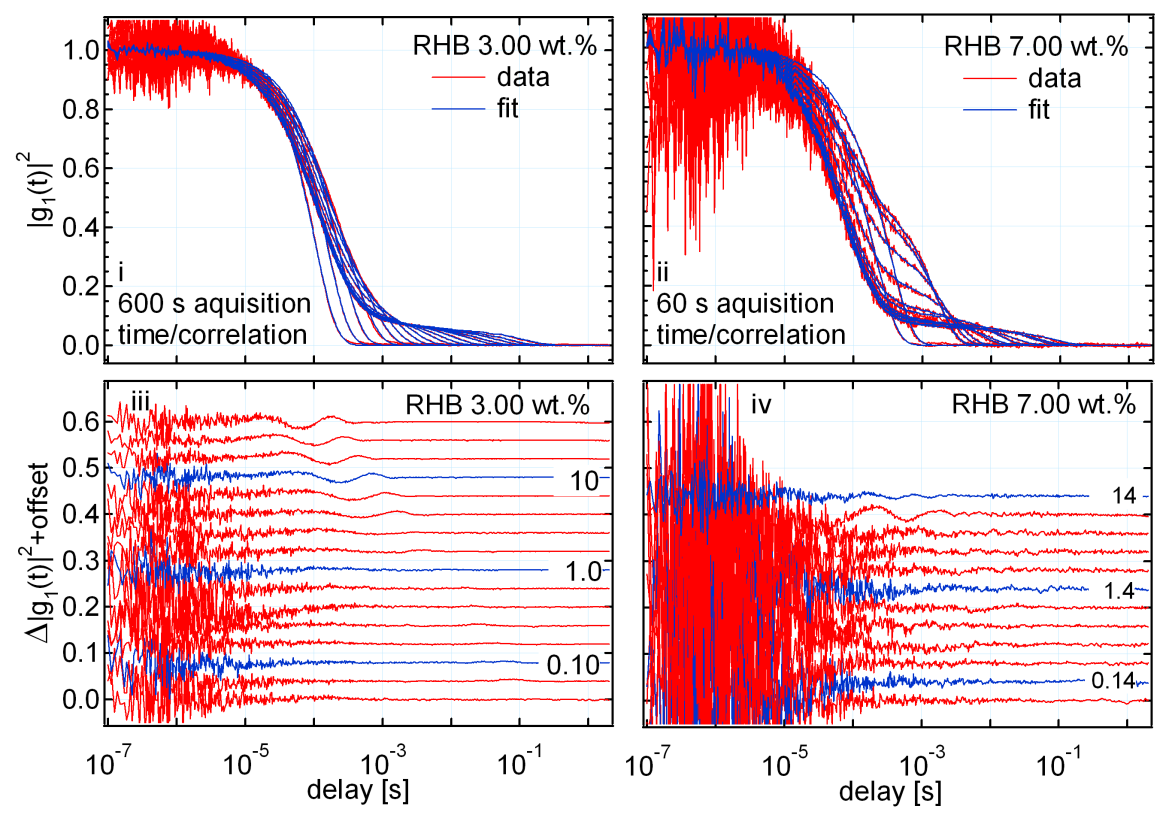

Figure 8.8: i-ii) Correlations with fit to a $\phi=3$ and 7 wt.\% RHB samples during a stepped strain rate test. iii-iv) Fit residuals measured with apparent strain rate noted in some instances.
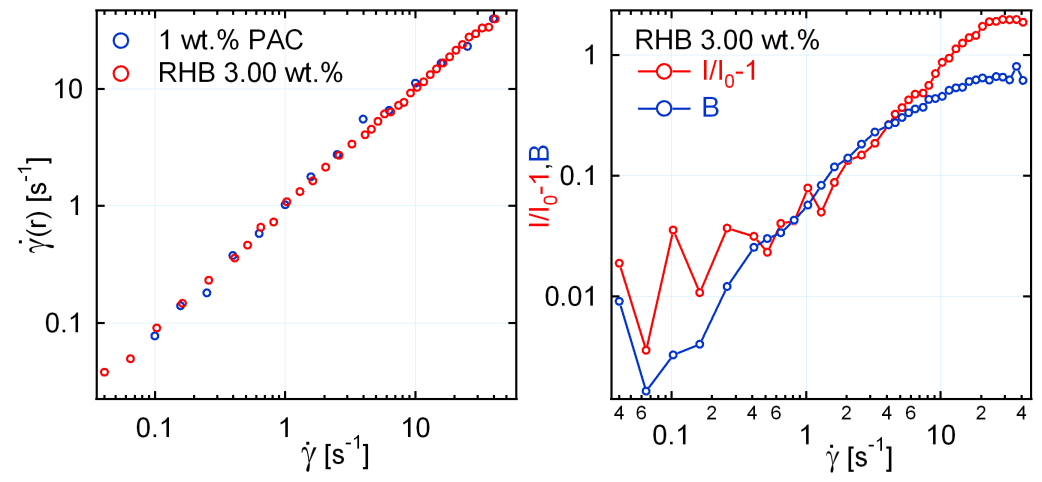

Figure 8.9: i) Fitted local strain rate $\dot{\gamma}(r)$ for both the polyacrylamide and $\phi=3$ wt. $\%$ RHB wormlike micelles solutions. ii) Fitted scattering parameter $B$ with measured scattered intensity for the $\phi=3$ wt.\% RHB sample. 

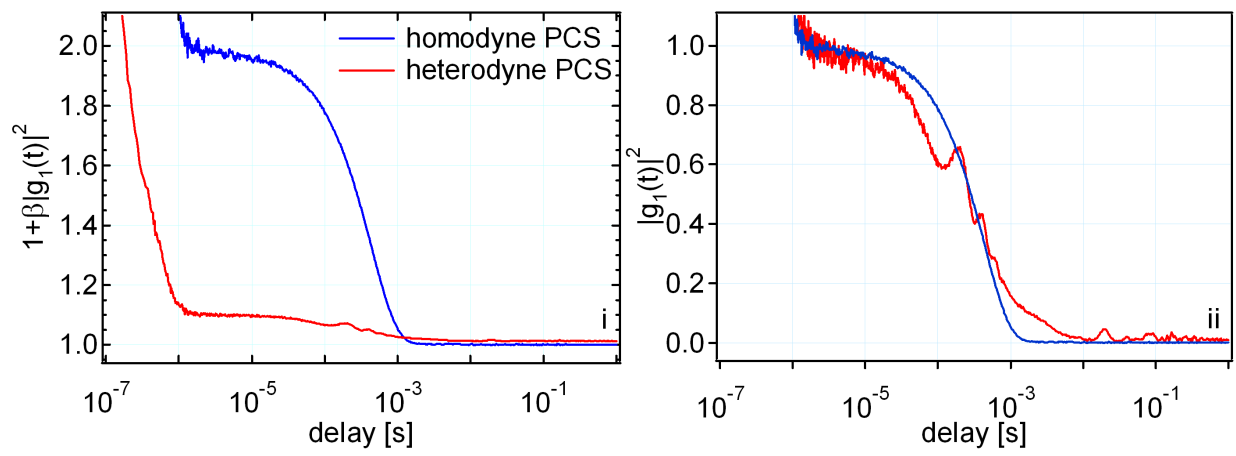

Figure 8.10: Measured homodyne and heterodyne PCS intensity correlations in flow conditions.

The fitted parameter $B$ is strongly correlated with the average excess scattered intensity, with the $\left[I / I_{0}-1\right] \propto B^{2}$ at higher strain rates.

An attempt was made to measure velocity using heterodyne PCS. In heterodyne PCS a reference beam is optically mixed on the surface of the detector with the light scattered by the sample. For the flow configuration outlined above and neglecting diffusion, the field correlation function is expected to be of the form [11]

$$
\left.\left|g_{1}(t)\right|=\exp \left[-\left(t / \tau_{\dot{\gamma}}\right)^{2}\right] \exp \left[-\left(t / \tau_{v}\right)^{2}\right] \times \Re \exp [i \mathbf{q} \cdot \mathbf{v}(r) t]\right) .
$$

The velocity dependence of the flow modulates the correlation function. To perform heterodyne PCS, the direction of the bidirectional beam splitter was reversed to give two input channels and a one output channel. The incident laser beam was split with a glass slab beam splitter, attenuated with a variable neutral density filter and directed into one of the input channels - this beam was the reference. Scattered light, collected from the sample under flow at $\phi=60 \mathrm{deg}$, was directed into the other input channel. The output was connected to a single PMT. By increasing or decreasing the level of attenuation of the reference, both homodyne and heterodyne PCS modes were accessible. Fig. $8.10 \mathrm{i}$ and ii shows typical raw and normalised intensity correlations measured in flow conditions. Surprisingly, heterodyning significantly decreases $\beta$. While some degree of oscillation is present in the heterodyne PCS correlation, this oscillation is not well described by Eqn. 8.12 possibly indicating that the heterodyne PCS technique is more subtle than is suggested in the literature.

The small angle $\theta \approx 0$ speckle pattern under flow conditions was observed directly by projecting the pattern onto a distant screen. For the Newtonian suspensions, the speckle pattern appeared to circulate. The observed direction of circulation and apparent velocity of the speckles in three flow conditions is shown in Fig. 8.11. Here the beam is directed along the $\hat{\mathbf{k}}$ axis and flow is in $-\hat{\mathbf{i}}$ direction. The disc centred at $x=y=0$ indicates the position of the unscattered beam. By disconnecting the bob from the rheometer and rotating the bob and cup together, the behaviour of the speckle pattern in the absence of 


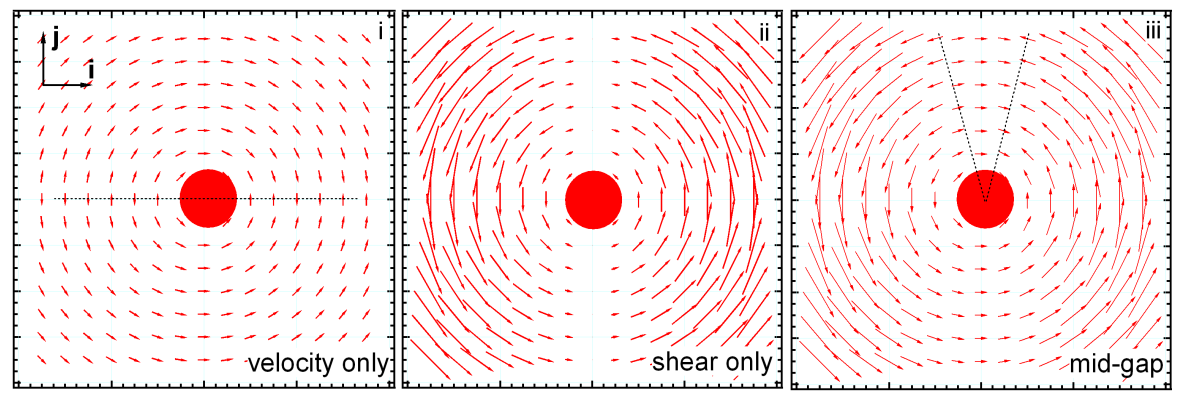

Figure 8.11: Apparent velocity of the laser speckle at small angles in three flow conditions. Note that these figures are based on direct observation and are somewhat idealised.

shear could be observed. The speckles were observed to circulate anticlockwise for $+y$ and clockwise for $-y$. The velocity of the speckles appeared to be fairly uniform. The bob was reconnected and the speckles pattern observed with the beam positioned at the outer wall of the gap. At the outer wall, the velocity of the fluid is close to zero - here the observed behaviour could be expected to be dominated by shear flow effects. The speckle pattern is observed to circulate anti-clockwise. The apparent velocity of the speckles increased with increasing $|x|$. Finally, the beam was shifted into a mid-gap position. The observed speckle pattern seen in Fig. 8.11 iii appeared to be a superposition of speckle motion seen in Fig. 8.11 i and 8.11 ii. Eqn. 8.4 describes the behaviour of the time correlation when transit effects can be neglected. At small angles, transit times cannot be neglected as $q$ is small. Furthermore, Eqn. 8.4 says nothing about the apparent space-time correlation observed in the speckle pattern. This statistics of the space-time correlation will be examined in detail in Chapter 9.

\subsubsection{Ellipsometry}

Shear induced optical anisotropy was measured using transmission ellipsometry. The vorticity axis is a principal direction in the cylindrical-Couette geometry. The polarisation state of light directed down this axis is only influenced by anisotropy in the two mutually perpendicular refractive indices. From measurements of the change in polarisation state, it is possible to infer $\Delta n=n_{11}-n_{22}$ - the difference between the refractive indices, and the angle $\chi$ - the orientation of these perpendicular axis with respect to the lab frame, and through the stress-optic law $\sigma=\sigma_{12}$ and $N_{1}$.

Measurements of optical anisotropy were made here using birefringence modulation ellipsometry [137]. A non-standard polariser-sample-modulator-analyser configuration was used in order to avoid modulating the polarisation state at the scattering volume. Following the conventions of reflection ellipsometry, a plane of incidence which contains both the incident and reflected beams, is defined. An $s$-direction is defined as perpendicular to this plane. Both the polariser and modulator were fixed in the $s$-direction. The analyser was 
rotated about the beam through an angle of $A=45 \mathrm{deg}$ with respect to the $s$-direction. Using the Mueller matrix/Stokes vector polarisation algebra (discussed in Chapter 3), representing the sample as an anisotropic linear retarder with orientation $\chi$ and retardation $\delta$ and neglecting the effects of reflecting surfaces and window birefringence, the time varying intensity at the detector for this configuration is

$$
I(t)=\frac{1}{4}\left[I_{0}+\cos \delta(t) \cos 2 \chi \sin 2 \chi(\cos \delta-1) \sin \delta(t) \sin (2 \chi) \sin (\delta)\right],
$$

where $\delta(t)=\mathcal{A} \sin (\omega t)$ is the time dependent phase modulation introduced by the birefringence modulator. The retardation $\delta$ is related to the difference in refractive indices by

$$
\delta=\frac{2 \pi \Delta n h}{\lambda}
$$

where $h$ is the optical path length and $\lambda$ is the wavelength of the incident light.

The resulting signal contains $\mathrm{DC}$ and $\mathrm{AC}$ components. The amplitude of components at the modulation frequency $(\approx 50 \mathrm{kHz}) \mathrm{AC}_{1}$ and twice this frequency $\mathrm{AC}_{2}$ are measured on a 2 channel lock-in amplifier. The DC component is measured separately and the AC/DC ratio determined:

$$
\begin{aligned}
\frac{A C_{1}}{D C} & =\frac{G_{1} J_{1}(\mathcal{A}) \sin (2 \chi) \sin (\delta) \cos \left(\phi_{\omega}\right)}{1+J_{0}(\mathcal{A}) \cos 2 \chi \sin 2 \chi(\cos \delta-1)}, \\
\frac{A C_{2}}{D C} & =\frac{G_{2} J_{2}(\mathcal{A}) \cos 2 \chi \sin 2 \chi(\cos \delta-1) \cos \left(\phi_{2 \omega}\right)}{1+J_{0}(\mathcal{A}) \cos 2 \chi \sin 2 \chi(\cos \delta-1)} .
\end{aligned}
$$

Here $G_{1}$ and $G_{2}$ are relative gain factors related to the gain of the $\mathrm{AC}$ and DC amplifier circuits and $\phi_{\omega}$ and $\phi_{2 \omega}$ are lock-in phases. The dependence on $\chi$ and $\delta$ in the DC term can be eliminated by choosing the modulation amplitude $\mathcal{A}$ such that $J_{0}(\mathcal{A})=0$. Dividing the ratios by experimentally determined calibration factors $G_{1} J_{1}\left(\mathcal{A}_{0}\right)$ and $G_{2} J_{2}\left(\mathcal{A}_{0}\right)$ gives the parameters of interest $x$ and $y$ :

$$
\begin{aligned}
& \frac{1}{G_{1} J_{1}\left(\mathcal{A}_{0}\right)} \frac{A C_{1}}{D C}=x=-\sin 2 \chi \cos 2 \chi(\cos \delta-1), \\
& \frac{1}{G_{2} J_{2}\left(\mathcal{A}_{0}\right)} \frac{A C_{2}}{D C}=y=-\sin 2 \chi \sin \delta,
\end{aligned}
$$

where it has been assumed that $\cos \left(\phi_{\omega}\right)=\cos \left(\phi_{2 \omega}\right)=-1$. Following observations made by Fuller in a closely related system, $[\mathrm{CPCl}]=0.1 \mathrm{M}$ and $[\mathrm{NaSal}]=0.06 \mathrm{M}$ in water (see [13] or [104]), it was assumed that $\chi$ was initially positive and that $\delta$ initially decreased from $\delta=0 \mathrm{deg}$ (implying that the stress-optical coefficient is negative) with increasing strain rate for the RHB system examined here and the lock-in phases were set accordingly. Physical reasons for a negative stress-optical coefficient in wormlike micelles solutions are addressed in [138]. This assumption was not explicitly tested. If necessary, $x$ and $y$ can be inverted to give $\chi$ and $\delta$. This involves solving a quadratic equation which has two pairs of roots. Only one pair of roots is physical. 

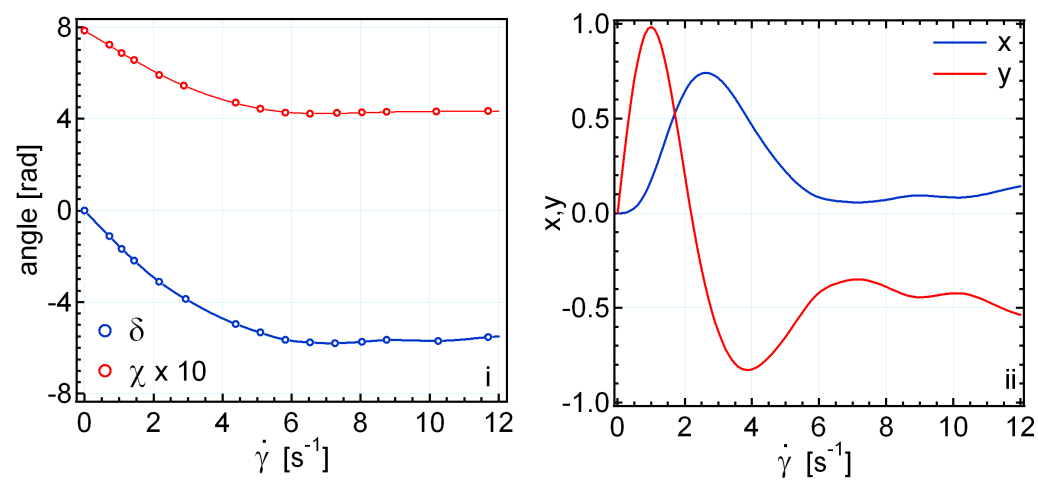

Figure 8.12: Measured birefringence [44 for a sheared $[\mathrm{CTAB}]=[\mathrm{KBr}]=0.3 \mathrm{M}$ aqueous wormlike micelles solution and ii) $x$ and $y$ calculated from the data in i.

The polarising optics configuration used here is most similar to that used in conventional reflection ellipsometry. Because the dependence of the measured parameters $x$ and $y$ on $\chi$ and $\delta$ is likely to be unfamiliar, the behaviour of these parameters is demonstrated for typical literature data. Lerouge and co-workers [44] examined shear induced optical anisotropy in a wormlike micelles solution composed from CTAB and $\mathrm{KBr}$ $[\mathrm{CTAB}]=[\mathrm{KBr}]=0.3 \mathrm{M}$ in water. From their data $\delta$ is calculated for $\lambda=633 \mathrm{~nm}$ and $h=0.05 \mathrm{~m}$. As illustrated in Fig. 8.12 i both $\chi$ and $\delta$ decrease linearly at low strain rate. $\chi$ decreases from $\chi=\pi / 4$ whereas $\delta$ decreases from $\delta=0$. At higher strain rates, both $\chi$ and $\delta$ are observed to plateau; this was correlated with the appearance of a stress plateau in the steady-state flow curve. Calculated $x$ and $y$ for Lerouge's data are shown in Fig. 8.12 ii. Based on these data it is expected $x \propto \dot{\gamma}^{3}$ and $y \propto \dot{\gamma}$ for small $\dot{\gamma}$.

The effect of the protected front reflecting gold mirrors on polarisation state was examined on a conventional birefringence modulation ellipsometer. The effect of a reflection from an isotropic non-depolarising surface on the polarisation state at a given angle of incidence and wavelength is completely described by the complex reflection ratio $r=r_{p} / r_{s}=\rho_{m} \exp \left[i \delta_{m}\right]$. This quantity describes the change in relative amplitude and phase of the incident $s$-polarised and $p$-polarised light. This quantity was measured for one of the two mirrors used as a function of angle of incidence at $\lambda=633 \mathrm{~nm}$. In the rheometer the mirrors reflect light at an angle of incidence of $\theta=45 \mathrm{deg}$. As illustrated in Fig. $8.13 \rho_{m}=0.974$ and $\delta_{m}=10.4^{\circ} \mathrm{deg}$ at this angle. With the polariser on the rheo-optics apparatus set at $P=0 \operatorname{deg}$ ( $s$-direction), the first mirror can be neglected. In the approximation that $\rho_{m}=1$ it can be shown using the Mueller matrix/Stokes vector algebra that the uncorrected measured quantities $x^{\prime}$ and $y^{\prime}$ are related to correct $x$ and $y$ values by a rotation through $\delta_{m}$

$$
\begin{aligned}
& x=x^{\prime} \cos \delta_{m}+y^{\prime} \sin \delta_{m}, \\
& y=y^{\prime} \cos \delta_{m}-x^{\prime} \sin \delta_{m} .
\end{aligned}
$$




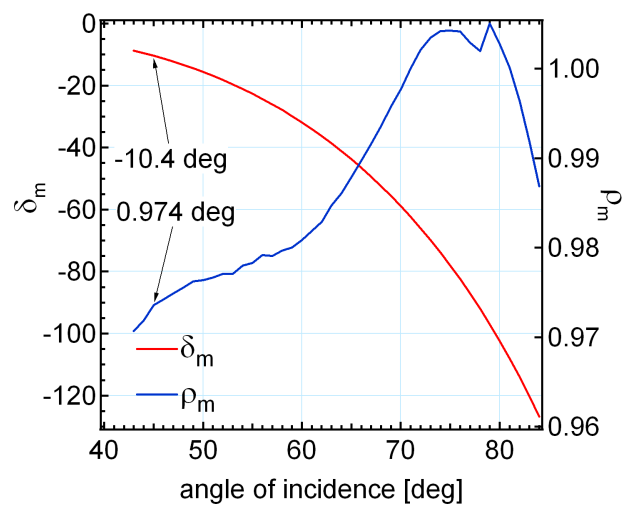

Figure 8.13: Variable angle ellipsometry measurements of a protected gold mirror at $\lambda=$ $633 \mathrm{~nm}$. These measurements were used to correct for the effect of one of the mirrors on the polarisation state.

Static window birefringence in the 'base' and the 'cap' of the optical cylindricalCouette was relieved by annealing the perspex windows at $T=70{ }^{\circ} \mathrm{C}$ for 150 hours. The birefringence in both the base and cap of the geometry was examined separately in transmission on the conventional birefringence modulation ellipsometer. Measurements were made in a standard polariser-modulator-sample-analyser configuration with the polariser orientated at $P=45 \mathrm{deg}$ and the modulator at $M=0 \mathrm{deg}$ as a function of the orientation of the analyser $A$. The windows (sample) were carefully orientated in the beam to match the orientation used in the rheo-optics configuration and measurements were made at the location of the gap midpoint. In the approximation that the windows can be treated as linear retarders with an orientation $\chi_{w}$ and phase shift $\delta_{w}$, the $y$ value is calculated to be

$$
y=\sin \delta_{w} \sin \left[2 A-2 \chi_{w}\right]
$$

Measured $y$ for both the cap and base along with estimated $\chi_{w}$ and $\delta_{w}$ for the two windows is given in Fig. 8.14 i. If $\chi_{w}$ was orientated at $n \pi / 2$ it can be shown that the correction to $x$ and $y$ takes the form of the correction applied for the protected gold mirror - this is not the case for the two windows here. Correcting the data for the effect of birefringence at an arbitrary orientation angle $\chi_{w}$ is not straightforward. Fortunately, as the measurements indicate, the phase shift is small. The anisotropy in the windows was observed to produce a non-zero signal in the rheo-optic configuration in the no shear condition. Measured values were in the range $x=0.0040-0.0080$ and $y=0.010-0.025$. Because the phase shift was known to be small and because $x$ and $y$ showed small amounts of variation, it was decided to treat these non-zero $x$ and $y$ values simply as an offset.

Dynamic window birefringence associated with stress induced anisotropy in the windows was investigated for a Newtonian fluid (water) over the range of torques applied in the rheo-optical measurements of the wormlike micelles at two polariser orientations. As illustrated in Fig. 8.14 ii the maximum average changes in $x$ and $y$ over the torque range 

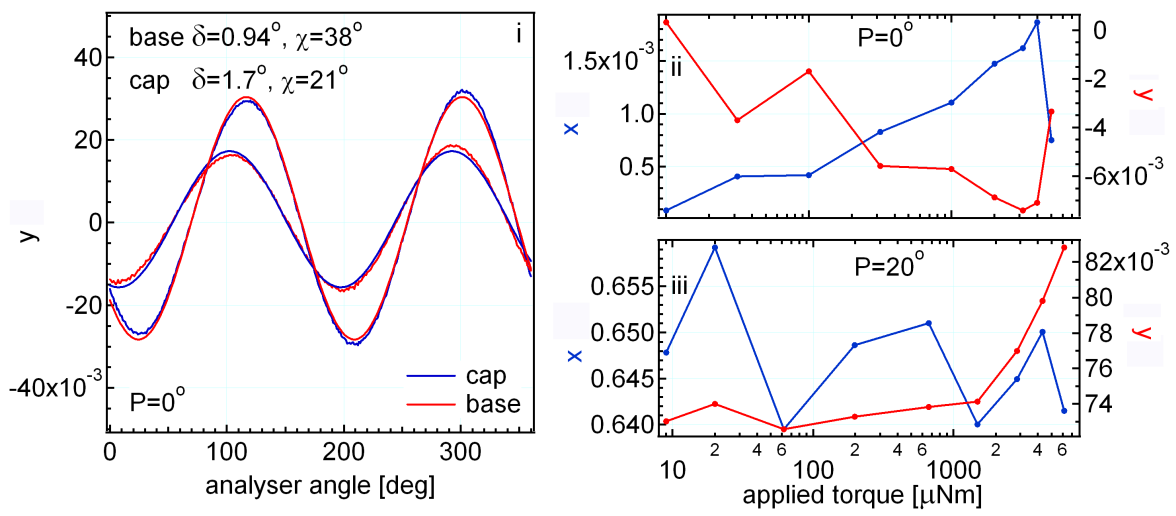

Figure 8.14: i) Measured 'static' or residual birefringence in the upper and lower window of the cylindrical-Couette flow-cell and ii) total stress induced birefringence in both windows over the range of torques applied during later experiments to the flow-cell.

examined was $\Delta x \approx 0.01$ and $\Delta y \approx 0.01$. The effect of dynamic window birefringence on the ellipsometry measurements is expected to be small and was neglected.

\subsection{Measurements and analysis}

\subsubsection{Homodyne PCS}

Samples were carefully loaded into the cylindrical-Couette and allowed to equilibrate for between 30-60 minutes. Following this, an equilibrium correlation $\left(\left|g_{1,0}(t)\right|\right)$ was acquired over 20 minutes at mid gap. Shear was applied and the beam was stepped through the gap from the outer wall. The samples were measured at 12 positions in the gap from approximately $r_{i}+13 d / 15$ to $r_{i}+2 d / 15$ in $100 \mu \mathrm{m}$ increments at each strain rate. The apparent strain rates were log-spaced at 5 /decade, then 10 /decade then 20 /decade over $3+$ orders of magnitude, with choice of range based on the steady-state flow properties. Total acquisition time at each position at each strain rate was $60 \mathrm{~s}$. The passage of small bubbles through the beam was observed to disrupt the correlations in a characteristic way. To counter the effect of bubbles $12 \times 5 \mathrm{~s}$ correlations were acquired in each $60 \mathrm{~s}$ acquisition interval; correlations affected by the passage of bubbles were automatically discarded and the remaining correlations averaged in the appropriate way (see [139]). Total measurement time for each sample was approximately 8 hours. Samples were observed to fracture (larger bubbles were observed in the sample) at a strain rate about half of that observed in the cone-plate geometry. The measured correlation was fitted to Eqn. 8.11 to determine both the local strain rate $\dot{\gamma}(r)$ and $B$. Fig. 8.15 i-vi shows the fitted local strain rate for the $\phi=$ 3 wt.\% to 8 wt.\% samples for each of the 12 locations examined within the gap, along with model curves displaced on the vertical log scale to make each set clearly visible. Model 


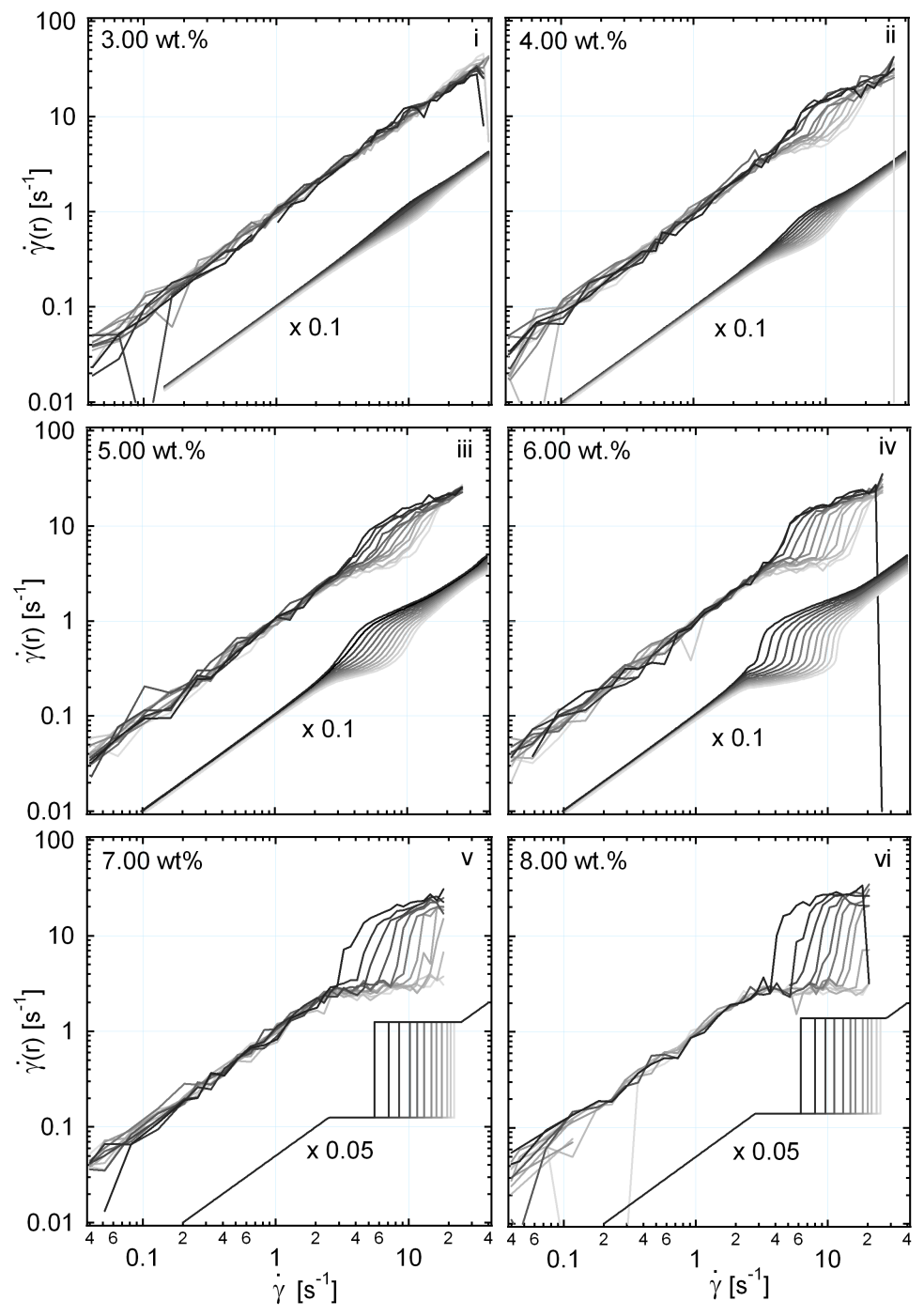

Figure 8.15: i-vi) Point-wise measurements and model values of the local strain rate $\dot{\gamma}(r)$ across the gap for the $\phi=3-8$ wt.\% RHB wormlike micelles solutions. The model values for the $\phi=3-6$ wt.\% samples were calculated from the flow curves shown in Fig. 8.3 whereas $\phi=7-8$ wt.\% were calculated using the lever rule. Each line corresponds to one of the 12 radial measurement positions with the lightest grey nearest the outer wall. 
data were calculated from steady-state flow behaviour or from the lever rule as follows. The stress in the cone-plate geometry has the angular variation

$$
\sigma_{\phi, \theta}=\sigma_{0} \operatorname{cosec}^{2}[\pi / 2-\theta] .
$$

where $\sigma_{\phi, \theta}(\theta)$ is the local stress and $\sigma_{0}$ is the stress at the surface of the cone. For the $\theta_{0}=1 \mathrm{deg}$ cone used for the mechanical rheometry measurement seen in Fig. 8.3 the angular stress variation across the gap is about $0.030 \%$ implying that $\sigma(\theta) \approx \sigma_{0}$. The radial variation of shear stress in the cylindrical-Couette geometry is given by

$$
\sigma_{\phi, r}(r)=\sigma_{0}\left[\frac{r}{r_{i}}\right]^{-2}
$$

For the rheo-optic geometry used here, the stress decreases by about $14 \%$ from the inner wall. Using the steady-state cone-plate flow curve measurements, the local strain rate in the gap of the cylindrical-Couette can be determined for an apparent strain rate given by the integral

$$
\dot{\gamma}=\frac{r_{i}}{r_{o}-r_{i}} \int_{r_{i}}^{r_{o}} \frac{\dot{\gamma}(r)}{r} d r
$$

The model curves in Fig. 8.15i-iv show the calculated local strain rate with apparent strain rate across the central region of the gap. The fitted local strain rate matches the calculated local strain rate reasonably well suggesting that $\dot{\gamma}(r)$ has been successfully extracted from the measured correlation. A stress plateau was observed in the steady-state flow curve for the $\phi=7$ wt. $\%$ and 8 wt. $\%$ wormlike micelles solutions - these samples are expected to shear band. For these samples, the local strain rate is calculated according to the lever rule, $\dot{\gamma}=x \dot{\gamma}_{H I}+(1-x) \dot{\gamma}_{L O}$, assuming that the fluid partitions into two homogeneous strain rate bands and assuming that the high strain rate band (of width $x$ ) forms at the moving wall. $\dot{\gamma}_{L O}$ was chosen from the onset of shear banding as shown by the optical data and $\dot{\gamma}_{H I}$ was chosen to give the best match between the optical and calculated data. Values of $\dot{\gamma}_{L O}=2.5 \mathrm{~s}^{-1}$ and $\dot{\gamma}_{H I}=25 \mathrm{~s}^{-1}$ and $\dot{\gamma}_{L O}=2.8 \mathrm{~s}^{-1}$ and $\dot{\gamma}_{H I}=28 \mathrm{~s}^{-1}$ were assumed for $\phi=7 \mathrm{wt} . \%$ and $8 \mathrm{wt} . \%$ samples respectively. The calculated local strain rate is shown in Fig. 8.15 v and vi. The calculated and measured data are not very comparable, suggesting that the lever rule model described above is too simplistic. Measurements of the average scattered intensity associated with the data in Fig. 8.15 are shown in Fig. 8.16. A general increase in the scattered intensity with strain rate is observed. A slight decrease is observed near the outer wall in the $\phi=8$ wt. $\%$ sample. This decrease was also observed in the $\phi=9$ wt. $\%$ and 10 wt.\% samples. This decrease in scattered intensity made it difficult to determine the strain rate accurately in the low strain rate band for these two samples in the minute allocated for data acquisition at each point in the gap.

The behaviour of the $\phi=7$ wt.\% sample over the apparent strain rates at which banding was expected was examined in more detail. Measurements were made at 12 positions in the gap at linearly spaced apparent strain rate increments. Fitted $\dot{\gamma}(r)$ is shown in Fig. 8.17 i along with the scattered intensity in Fig. 8.17 ii. In Fig. 8.18 i 

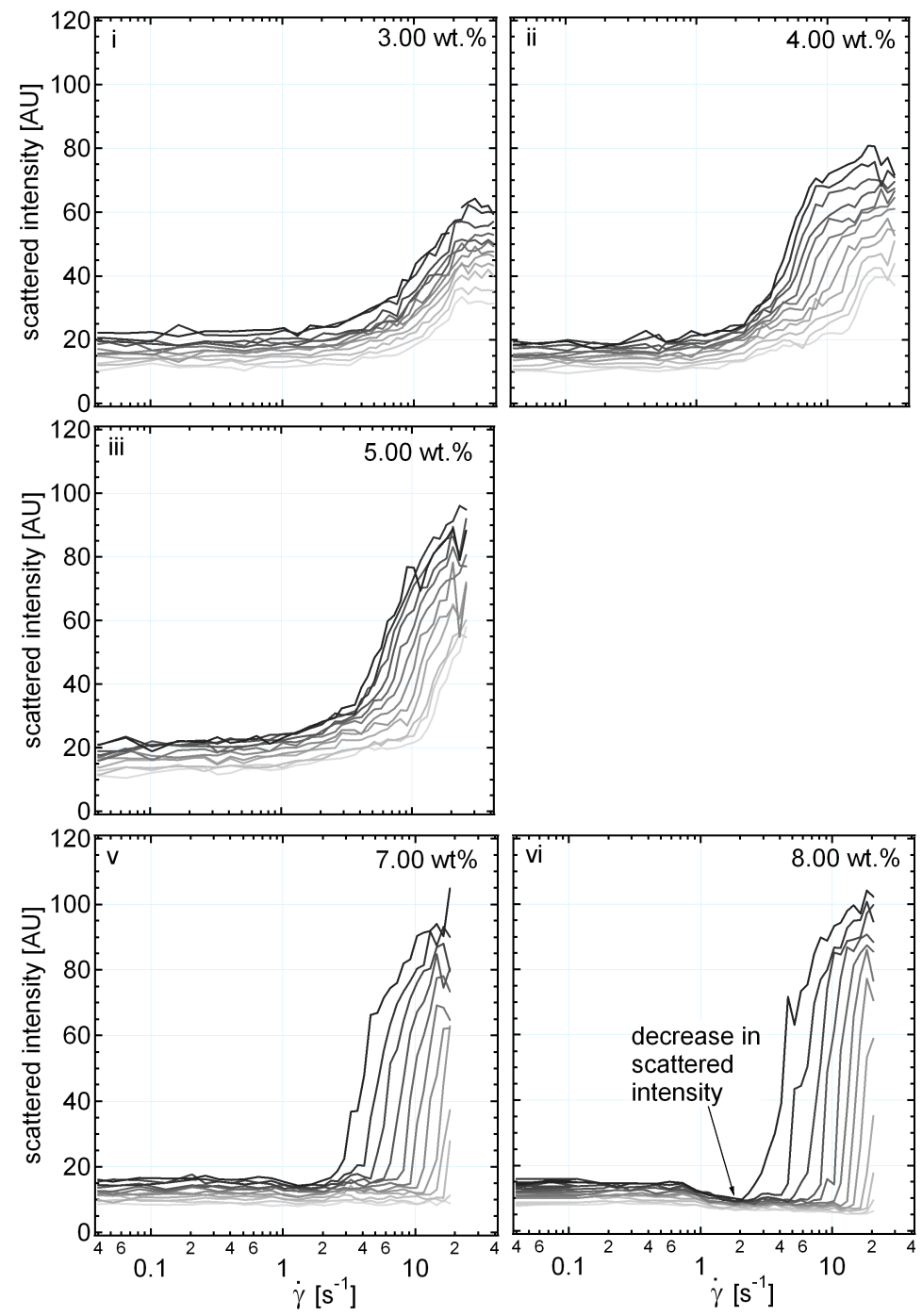

Figure 8.16: Point-wise measurements of the scattered intensity across the gap of the cylindrical-Couette for the $\phi=3-8 \mathrm{wt} . \%$ RHB wormlike micelles solutions. Each line corresponds to one of 12 radial measurement positions with the lightest grey nearest the outer wall. Note that the decrease in the scattered intensity observed in vi was more pronounced in the $\phi=9-10$ wt.\% samples (data not shown). 

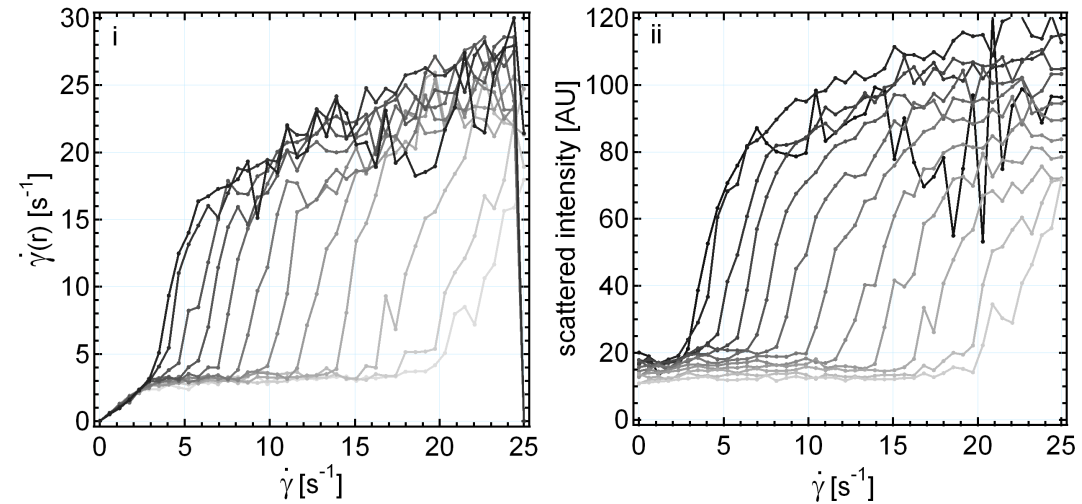

Figure 8.17: Point-wise measurements of the i) local strain rate $\dot{\gamma}(r)$ and ii) scattered intensity across the gap of the cylindrical-Couette for a $\phi=7 \mathrm{wt} . \%$ RHB wormlike micelles solution.
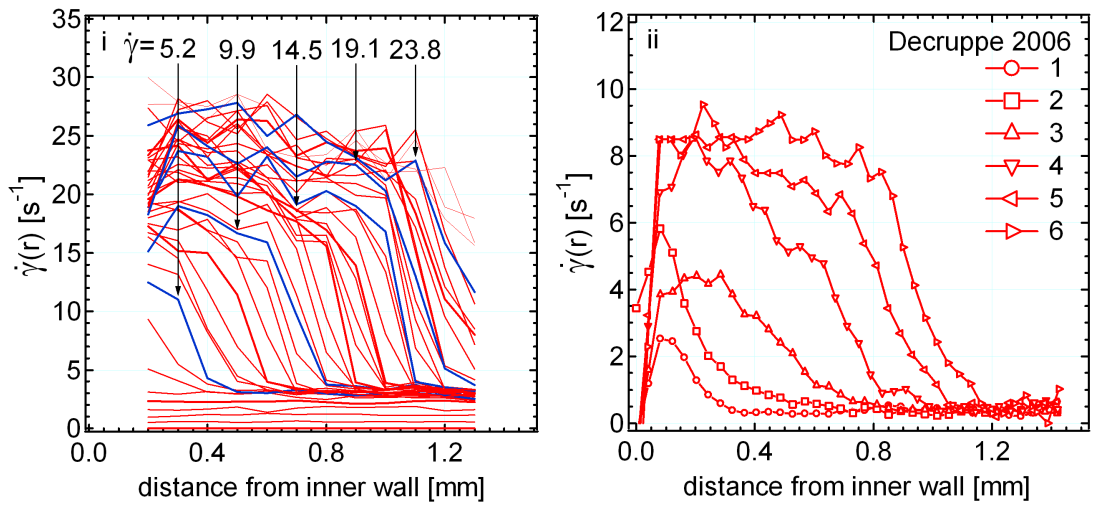

Figure 8.18: i) Point-wise measurements of the local strain rate across the gap of the cylindrical-Couette for the $\phi=7$ wt.\% RHB wormlike micelles solution. Each line corresponds to measurements made at a particular apparent strain rate (strain rate indicated in some cases). A clear indication of shear banding is apparent. ii) Differentiated ultrasonic velocimetry flow measurements of a $[\mathrm{CTAB}]=0.05 \mathrm{M}$ and $[\mathrm{NaSal}]=0.1 \mathrm{M}$ in water wormlike micelles solution showing the local strain rate across the gap in a cylindrical-Couette geometry from [120]. 
the data given in Fig. 8.17 i are replotted to show the local strain rate variation as a function of gap position. A higher strain rate band appears to grow out of a fairly stable low strain rate band and continues to grow without saturation with increasing $\dot{\gamma}$. A simple application of the lever rule predicts a fixed value for $\dot{\gamma}_{L O}$ and $\dot{\gamma}_{H I}$ respectively. While the data are consistent with shear banding, a constant value of $\dot{\gamma}_{H I}$ is not found. A similar lack of constancy for $\dot{\gamma}_{H I}$ is found elsewhere in the literature. Fig. 8.18 ii shows the local strain rate for a wormlike micelles solution of $[\mathrm{CTAB}]=0.05 \mathrm{M}$ and $[\mathrm{NaSal}]=0.1 \mathrm{M}$ in water between $\dot{\gamma}=1 \mathrm{~s}^{-1}$ and $\dot{\gamma}=6 \mathrm{~s}^{-1}$ measured by Decruppe and co-workers using ultrasonic velocimetry [120]. The rheology of this system was examined in Chapter 4. This system shows pronounced fluctuations for $\dot{\gamma}>6 \mathrm{~s}^{-1}$. The gradual emergence of a higher shear band out of the low strain rate band is observed, as was observed in the data here.

Because of the correlation between scattered intensity and local strain rate it is necessary to consider the possible influence of multiple scattering on the results presented here. The quantity

$$
\omega_{o}-\omega_{i}=\Delta \omega=\int_{r=r_{i}}^{r=r_{o}} \frac{\dot{\gamma}(r)}{r} d r
$$

is independent of the rheology of the sample. Optical measurements were made over $r=r_{i}+2 d / 15$ to $r=r_{i}+13 d / 15$ - the integral above cannot be calculated for the data presented here. It is possible to calculate $\Delta \omega$ for a Newtonian fluid over a reduced range - it seems reasonable that this integral should be fairly comparable to the integral of the experimental data over the same range if wall slip can be neglected. The experimental and calculated integral from the data presented later in Fig. $8.15 \mathrm{v}$ and $8.17 \mathrm{i}$ is shown in 8.19. The average measured integral exceeds the expected value by about $7 \%$ over the entire strain rate range suggesting a slight calibration error. The temporal coherence of multiple scattered light depends on the relative motion of multiple scattering centres. As a consequence, it is expected that multiple scattered light will dephase more rapidly than single scattered light (this property is exploited in DWS based techniques). It was demonstrated that the local strain rate can be measured at apparent strain rates where the scattering from the shear induced structures is minimal. If multiple scattering was affecting the result, it would be expected that the ratio of measured $\Delta \omega$ to calculated $\Delta \omega$ would increase with apparent strain rate as an increasing fraction of the integral includes light scattered from a region of higher turbidity. This was not observed. This suggests that multiple scattering is not significantly influencing the fitted local strain rate.

\subsubsection{Ellipsometry}

Ellipsometry data were acquired concurrently with the PCS measurements. Data were acquired at $1 \mathrm{~s}$ intervals. An average value over the 1 minute acquisition interval is reported here. The passage of small bubbles deflected the beam off the pinhole significantly reducing the measured $\mathrm{AC}$ and $\mathrm{DC}$ values. Data below a threshold DC value was excluded from the average. An oscillation in the AC and DC signals, coherent with the rotation of the bob was observed. It was assumed that this was associated with alignment errors of the 


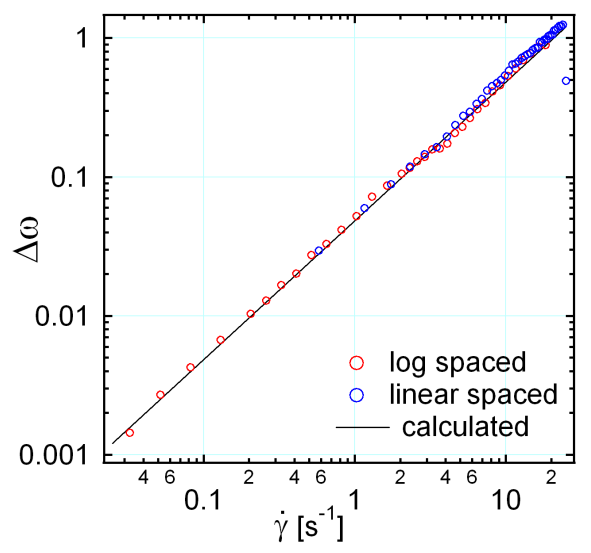

Figure 8.19: Measured and calculated $\Delta \omega$ for the $\phi=7 \mathrm{wt} . \%$ RHB sample. The measurement is comparable to the theoretical value suggesting that multiple scattering in the PCS measurements can be neglected.

homemade bob. Significant incoherent fluctuations were not observed - this is consistent with the mechanical rheometry measurements described earlier.

Fig. 8.20 i-vi shows $x$ and $y$ data corrected for the reflection from the second mirror (rotation through $\delta_{m}$ ) and for 'stray' birefringence introduced by the cap and base of the geometry (offset subtracted) as a function of apparent strain rate. These measurements show similar behaviour to that calculated for the Lerouge data discussed earlier. The measurements are observed to fan out with position. As stress is not uniform within the gap, some variation in anisotropy with position was expected.

The ellipsometry data shown in Fig. 8.20 were inverted to determine $\chi$ and $\delta$ and an 'optical' stress $\sigma_{o p}$ was calculated using Eqn. 8.26 adjusted for the expected mechanical stress variation with position in the gap

$$
\sigma_{o p}=\frac{1}{2 C} \times \frac{\delta \lambda}{2 \pi h} \sin [2 \chi] \times\left[\frac{r}{r_{i}}\right]^{-2}
$$

The stress-optical coefficient was determined from the relation $\sigma_{o p}=C \sigma$ from low shear optical and mechanical measurements. From the optical stress data it is seen that stressoptic law breaks down as the optical stress approaches the shear thinning region of the data. In all cases a significant drop in the optical stress is observed at a strain rate $\dot{\gamma}$ which depends on the position within the gap. Note that by definition $x^{2}+y^{2} \leq 1$. Uncorrected systematic errors cause $x^{2}+y^{2}>1$, and $x$ and $y$ cannot be inverted - this is the source of the gaps in the data. Measured $C$ is listed in Fig. 8.21. The stress-optic coefficient does not appear to show any systematic variation with concentration. The average $C$ value observed here was $C=-1.74 \times 10^{-7} \mathrm{~Pa}^{-1}$. Note again that it was assumed that the sign of $C$ was negative. Literature values are given in Table 8.1.

As discussed earlier, the $\phi=7$ wt. $\%$ sample was measured at 12 positions in the gap 

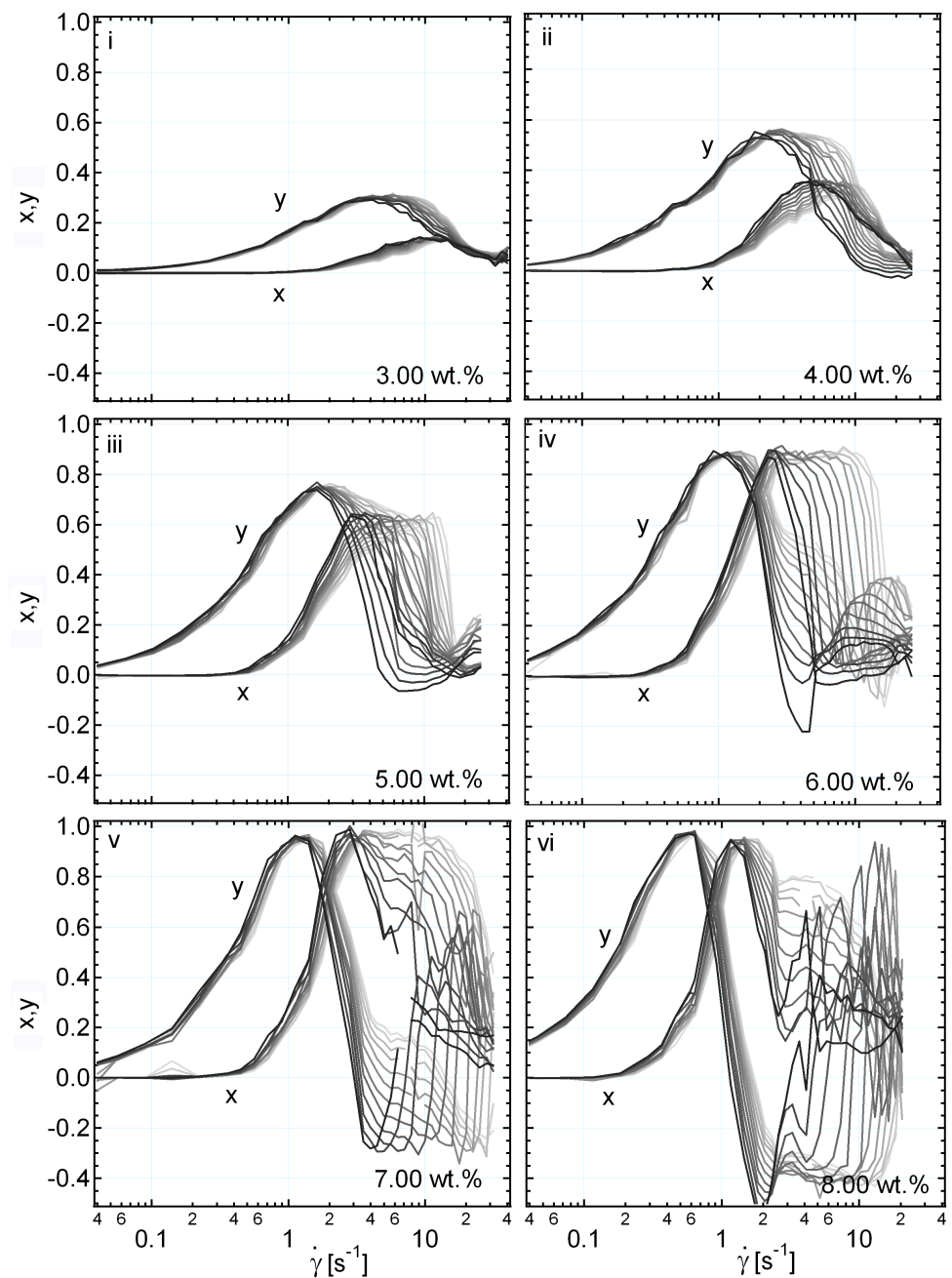

Figure 8.20: Point-wise measurements of $x$ and $y$ across the gap of the cylindrical-Couette for the $\phi=3-8$ wt.\% RHB wormlike micelles solutions. Each line corresponds to one of 12 radial measurement positions with the lightest grey nearest the outer wall. 

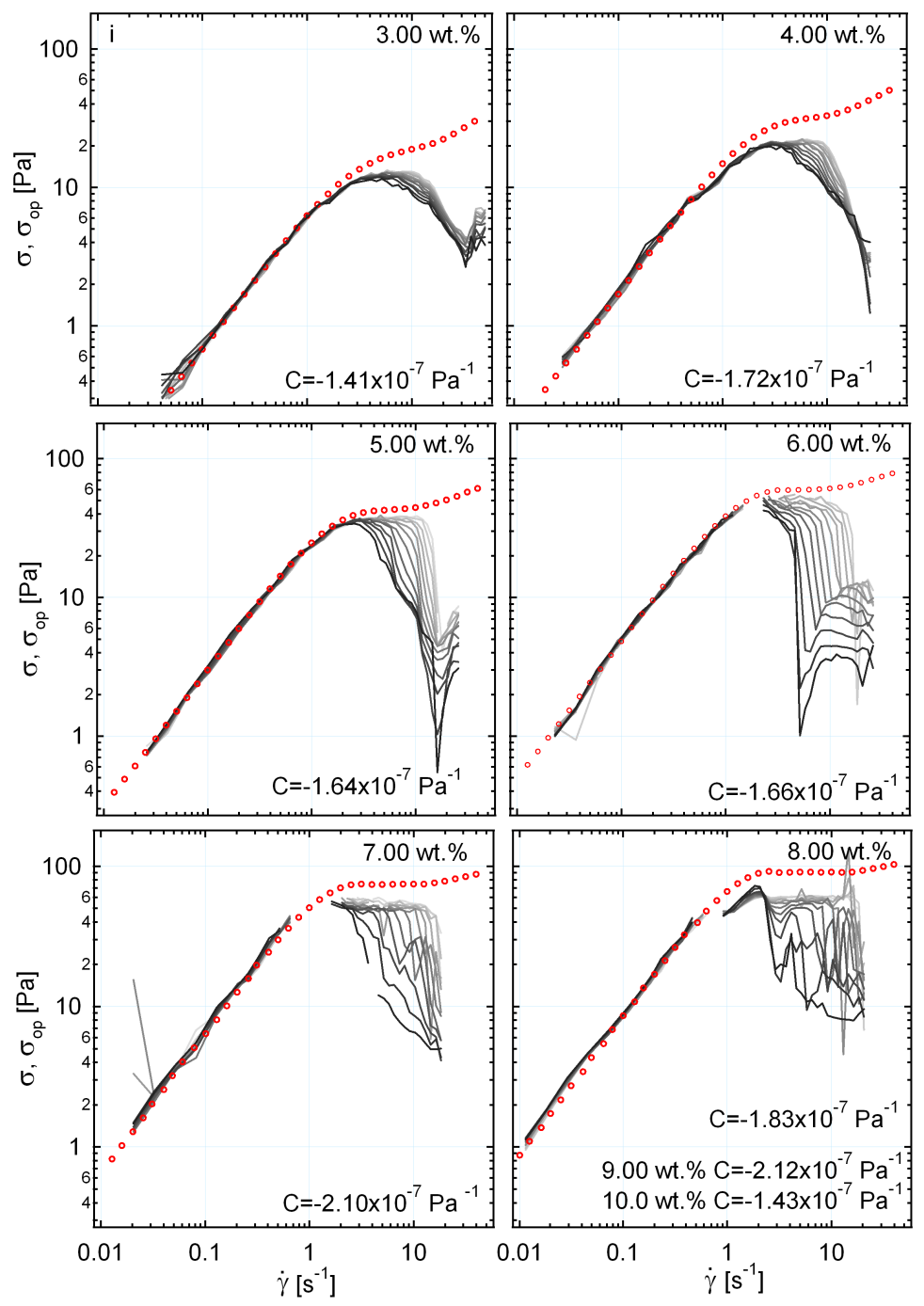

Figure 8.21: Measured optical shear stress $\sigma_{o p}$ assuming negative roots $\delta_{-}$and $\chi_{-}$(solid lines) with measured mechanical stress (open circles) for the $\phi=3-8$ wt.\% RHB wormlike micelles solutions. Each line corresponds to one of 12 radial measurement positions with the lightest grey nearest the outer wall. 


\begin{tabular}{|l|l|l|}
\hline system & condition & $\mathrm{C}\left[\mathrm{Pa}^{-1}\right]$ \\
CTAB $|\mathrm{KBr}|$ water & $0.3 \mid 0.3 \mathrm{M}$ & $-3.1 \times 10^{-8}[138]$ \\
CTAB $|\mathrm{KBr}|$ water & $0.3 \mid 0.3 \mathrm{M}$ & $+3.7 \times 10^{-8}[40]$ \\
CTAB $|\mathrm{NaSal}|$ water & $0.03 \mid 0.23 \mathrm{M}$ & $-3.49 \times 10^{-7}[123]$ \\
$\mathrm{CTAB}|\mathrm{NaSal}|$ water & $0.03 \mid 0.23 \mathrm{M}$ & $+5.74 \times 10^{-7}[124]$ \\
$\mathrm{CTAB}|\mathrm{NaSal}|$ water & $0.03 \mid 0.23 \mathrm{M}$ & $-2.77 \times 10^{-7}[140]$ \\
$\mathrm{CPCl}|\mathrm{NaSal}|$ water & $0.1 \mid 0.06 \mathrm{M}$ & $-2.3 \times 10^{-7}[13]$ \\
$\mathrm{CPCl}|\mathrm{NaSal}| \mathrm{NaCl} \mid$ water & {$[\mathrm{CPyCl}] /[\mathrm{NaSal}]=2 \mid[\mathrm{NaCl}]=0.5 \mathrm{M}$} & $-1.74 \times 10^{-7}[$ here, -ve assumed] \\
\hline
\end{tabular}

Table 8.1: Stress-optic coefficient reported for wormlike micelles solutions in the literature. The difference in signs appears to be associated with different formulations of the stressoptic rule. $C$ is negative if it is assumed that $2 C \sigma_{x y}=\Delta n \sin 2 \chi$ and positive it if is assumed that $2 C \sigma_{y x}=\Delta n \sin 2 \chi$.

at linearly spaced strain rate increments using ellipsometry. The $x$ and $y$ measurements were inverted and both the negative and positive roots are shown in Fig. 8.22, In terms of $x$ and $y$ the roots are:

$$
\begin{aligned}
& \delta_{ \pm}=\tan ^{-1}\left[\frac{-2 y a_{ \pm}}{1+y^{2} a_{ \pm}^{2}}, \frac{y^{2} a_{ \pm}^{2}-1}{1+y^{2} a_{ \pm}^{2}}\right], \\
& \chi_{ \pm}=-\frac{1}{2} \sin ^{-1}\left[\frac{y}{\sin \delta_{ \pm}}\right]
\end{aligned}
$$

where

$$
a_{ \pm}=\sqrt{-\frac{y^{2}-2 \pm 2 \sqrt{1-x^{2}-y^{2}}}{y^{4}+4 x^{2}}} .
$$

It is noted that $\delta_{-}$undergoes a sharp decrease at a strain rate correlated with position whereas the $\delta_{+}$values are clustered around $\delta=-\pi$. $\chi_{-}$is observed to decrease with increasing strain rate whereas $\chi_{+}$is seen to increase. To better understand the relationship between these roots, $x$ and $y$ were calculated for a grid of $\delta$ and $\chi$ values over intervals $0<\delta<2 \pi$ and $0<\chi<\pi / 8$. This grid was inverted to give $\delta_{ \pm}$and $\chi_{ \pm}$. The inverted grid of roots is shown in Fig. 8.23 i. Note that the inverted data are mapped into an interval $-\pi<\delta<\pi$ - when the input $\delta$ exceeds $\pi$ it is mapped to $-\pi<\delta<=\pi$. $|\delta|$ is observed to exceed $\pi$ in our experimental data - continuity arguments were applied to determine the correct $\delta$ in these cases. While the negative roots map to the correct value of $\delta$ and $\chi$ the positive roots are clustered around $\delta= \pm \pi$. $\delta$ depends on $h$ and $\lambda$ - the Author can think of no physical reason why $\delta$ would cluster around $\pi$. The negative root is only physical when

$$
[4 \chi-\pi]^{2}+[\delta-\operatorname{sign}(\delta) \pi]^{2}>[\pi / 2]^{2} .
$$

The boundary between positive and negative roots is illustrated in Fig. 8.23 i. This condition is quite restrictive. This suggests that the negative root is physical for the data presented here. 

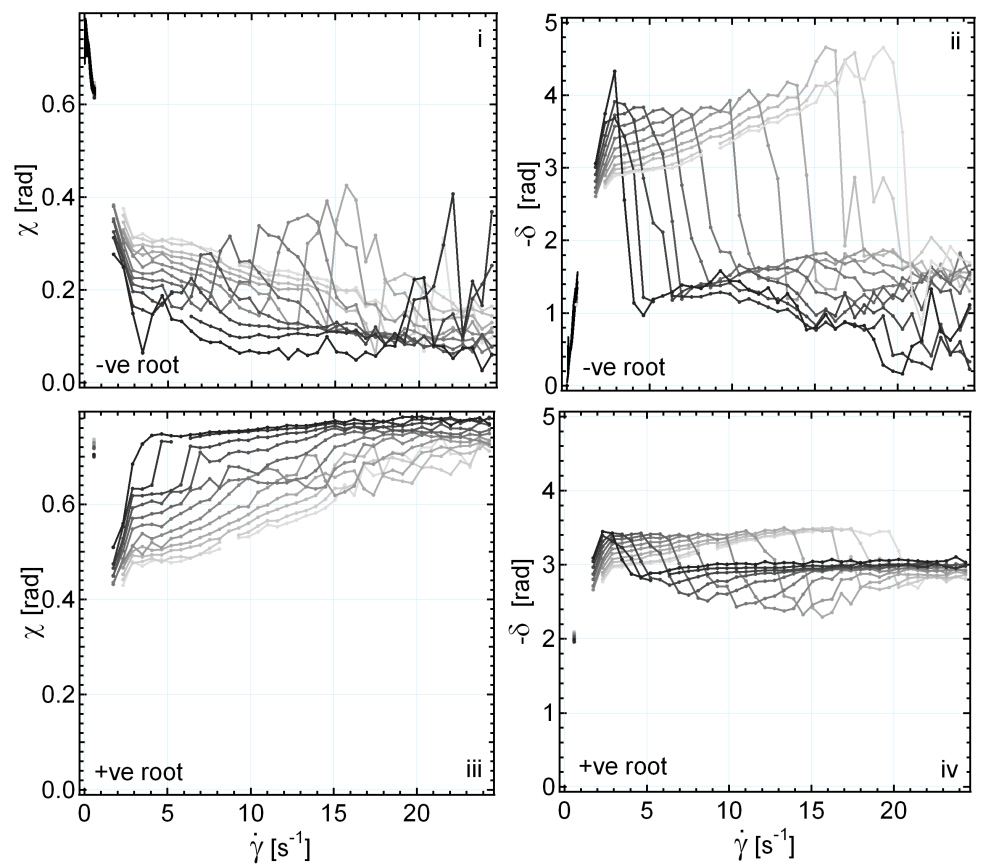

Figure 8.22: Point-wise measurements of $\chi$ and $\delta$, determined by inverting $x$ and $y$, across the gap of the cylindrical-Couette for the $\phi=7 \mathrm{wt} \%$ RHB wormlike micelles solution. Each line corresponds to one of 12 radial measurement positions with the lightest grey nearest the outer wall. i-ii) Correspond to negative roots of $x$ and $y$ and iii-iv) to positive roots of $x$ and $y$.
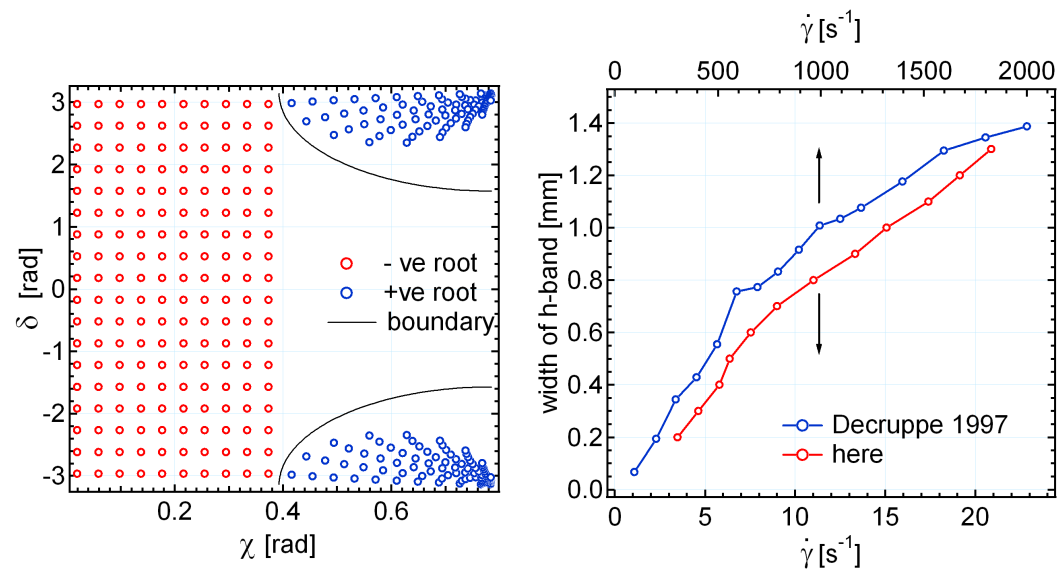

Figure 8.23: i) An examination of the relationship between the positive and negative roots of $x$ and $y$. ii) Width of the birefringent band with a comparison to the width measured in [40] in another wormlike micelles solution. 

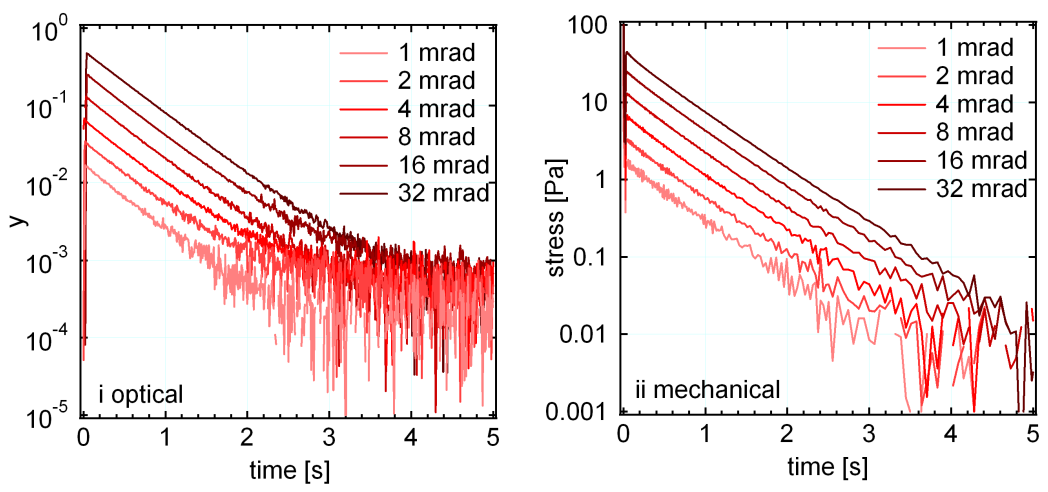

Figure 8.24: i) Mechanical measurements in the cone-plate geometry and ii) optical measurements in the cylindrical-Couette geometry of the $\phi=4$ wt.\% RHB wormlike micelles solution subjected to a sudden deformation. These measurements demonstrate that the optical configuration discussed here could be used to measure stress relaxation.

The width of the 'high' strain rate band with $\dot{\gamma}$ is calculated from the midpoint $\delta$ value at the transition from low birefringence to high birefringence shown in Fig. 8.23 ii. These data are compared to those given in [40. In both cases, the width of the high shear band does not increase linearly with apparent strain rate as predicted by the lever rule.

For $\chi \approx 45 \mathrm{deg}$ and $\delta \approx 0 \mathrm{deg}, y \propto G(t)$. This is illustrated in Fig. 8.24 where the stress measured after the application of deformation of $1,2,4,8,16$ and $32 \mathrm{mrad}$ using a cone/plate geometry is compared to the $y$ value measured in the rheo-optical configuration. Because of birefringence modulation ellipsometry temporal resolution, the technique could be used to examine the high frequency (short time) rheological behaviour. Because the rheometer required a minimum of $20 \mathrm{~ms}$ to apply to a deformation, this was not pursued.

\subsection{Discussion}

The data shown in Fig. 8.17 i, 8.22 ii and 8.17 ii are mapped to the surfaces shown in Fig. 8.25 i, ii and iii. Fig. 8.25 ii shows a large increase in $\delta$ value which is correlated with strain rate suggesting that for this system at least, strain rate bands are 'birefringence' bands. However, strain rate is also correlated with turbidity. Scattering structures may modify the change in polarisation state through form birefringence and dichroism. Non-zero dichroism has been observed before in non-absorbing wormlike micelles systems indicating that scattering can influence the measured polarisation state. In [104] Fuller and coworkers investigated the optical anisotropy in a wormlike micelles solution composed from $\mathrm{CPCl}$ and $\mathrm{NaSal}([\mathrm{CPCl}]=0.1 \mathrm{M},[\mathrm{NaSal}]=0.06 \mathrm{M})$ in water as a function of position in an optical configuration similar to that used here. An increase in $\Delta n$ was observed 

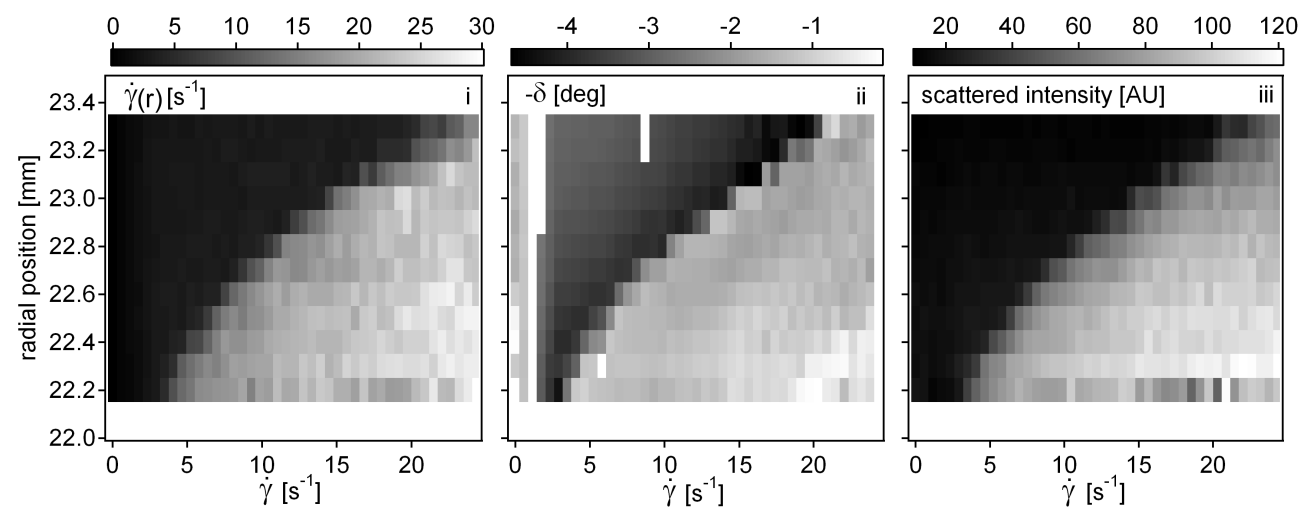

Figure 8.25: Correlation between i) strain rate, ii) birefringence and iii) scattered intensity in the $\phi=7$ wt.\% RHB wormlike micelles solution.

near the moving wall on the stress plateau. As discussed in Chapter 3 (and by Fuller and co-workers in [104]) the contribution of 'extrinsic' form birefringence to $\delta$ is always positive whereas the contribution of the 'intrinsic' stress induced birefringence may be positive or negative depending on the system. A significant increase in $\delta$ was observed here which was correlated with turbidity, suggesting that scattering processes are affecting the change in polarisation state here. It is noted that other workers have observed an apparent decrease in $\Delta n$ (see for example [44]). Using the formalism discussed [12] it appears possible to include the effects of (possibly non-coaxial) intrinsic and extrinsic birefringence and dichroism on the change in polarisation state. In this case, $x$ and $y$ will show an additional dependence on the orientation angle, birefringence and dichroism of the extrinsic contribution. It may be possible to distinguish the intrinsic and extrinsic contributions by varying the orientation angle of the polariser. This was not pursued here as the measurements would have required an additional correction for the first mirror which can only be neglected if the polariser is orientated in the $s$ or $p$ direction. Failing this, spectroscopic ellipsometry could be used. As the dependence on an extrinsic contribution was not included in the formulation of $x$ and $y$ it is not possible to conclude from our optical measurements that the high strain rate band is necessarily highly ordered or nematic only that it exhibits a different optical anisotropy.

What is perhaps more interesting is the correlation between turbidity and strain rate. Turbidity in sheared wormlike micelles has been observed on numerous occasions (Table 3.2). This turbidity is often attributed to the formation of shear induced structures. Shear induced turbidity has also been observed in polymer solutions. In polymer solutions this turbidity, associated with the formation of orientated aggregates and attributed to coupling between concentration fluctuations and stress, is often found to correspond to the onset of shear thinning as was observed here [56] [141] [142] (this correspondence between shear induced turbidity and shear thinning in wormlike micelles does not appear to be universal, see for example [66]). Concentration coupling to flow was predicted to increase 
the tendency of a complex fluid to shear band and to predict a concentration difference in the banded regions 22$] 73$.

In [103] Holmes, Lopez-Gonzalez and Callaghan examined the proton NMR spectra of a shear banding wormlike micelles solution closely related to that studied here composed of [cetylpyridinium chloride] $/$ [sodium salicylate] $=2$ at $\phi_{\mathrm{CPCl}+\mathrm{NaSal}}=10 \% \mathrm{w} / \mathrm{V}$ with $[\mathrm{NaCl}]=0.5 \mathrm{M}$ in $\mathrm{D}_{2} 0$ at equilibrium and compared it to that measured under shear flow conditions. The spectral line of protons on the aliphatic tail of the $\mathrm{CPCl}$ molecule was composed of two components: a broad spectral peak indicating the presence of slower or more constrained molecular motion and a narrower peak indicating faster or more mobile behaviour. Spatially resolved NMR spectroscopy did not clearly show a concentration difference between the low shear and high shear bands; it did, however, indicate a fractional increase in the constrained to mobile population in the higher strain rate band. This result was attributed to the shear induced alignment of wormlike micelles in the high shear band. However, this observation is also compatible with the formation of orientated aggregates - since any mechanism which slows the molecular reorientation or which reduces the symmetry of reorientation from spherical to non-isotropic can have the effect of broadening the proton NMR linewidth.

It is emphasised that it is not doubted that the higher strain rate band is highly ordered as has been clearly demonstrated by NMR and SANS - only that it was not possible to conclude with confidence that the anisotropy banding observed for the system examined here was not due to scattering from shear induced structures. These results may illustrate that when heterogeneity is present, interpretation may not be straightforward. 


\section{Chapter 9}

\section{Using simulation to investigate the statistical properties of dynamic speckle}

\subsection{Introduction}

In Chapter 8, a rather curious observation was made: the dynamic laser speckle pattern produced by scattering centres embedded in a flowing fluid appeared to circulate slowly about the unscattered transmitted beam. This apparent circulation showed a dependence on the type of flow present. In this chapter, this phenomenon is quantitatively studied with a view to developing a homodyne PCS/ellipsometry based experimental technique capable of resolving both flow and order independently at small angles using a camera based detection system.

The space-time dynamics of laser speckle pattern depend on both the space-time dynamics of the scattering centres and on the optical configuration. If this relationship is well understood, the dynamic behaviour of scattering centres can be inferred from measurements of the statistical properties of the laser speckle. This is the basis of the optical technique: photo correlation spectroscopy.

Conventionally, in PCS, a time intensity correlation is measured at a single point in the speckle field. Generally, measurements are made using a high sensitivity low latency detector (such as a photomultiplier or an avalanche photo diode) and autocorrelation is performed in hardware using a correlator. If multiple detectors are used, a space-time cross-correlation can be calculated. These cross-correlations can reveal motion not evident in the time autocorrelation; for example, the direction of flow can be inferred. CMOS and CCD sensor arrays are therefore potentially useful for studying the space-time dynamics of laser speckle. Historically, these sensor arrays have been avoided in dynamic light scattering because of their low sensitivity and high latency and because of significant 
storage and post-processing requirements associated with calculating correlations in software. Recent improvements in both sensor technology and CPU processing power have made camera based dynamic light scattering increasingly practical for studying space-time statistics of the dynamic speckle pattern.

Sensor arrays are already utilised in small angle light scattering (SALS) and can be used in ellipsometry (imaging ellipsometry) to measure structure and ordering in complex fluids near and in transmission. Clearly, a technique capable of resolving flow at small angles would complement these camera based rheo-optical techniques. As illustrated in the last chapter, the characteristic coherence time of laser speckle depends on flow behaviour. In particular, it was indicated that the characteristic coherence times associated with velocity and strain rate were $\tau_{v}=\left[v /\left(L \sqrt{2}^{-1}\right.\right.$ and $\tau_{\dot{\gamma}}=[2 k L \dot{\gamma} \sin (\theta / 2) \cos (\phi) / 2]^{-1}$. For the physically reasonable choices $v=1 \mathrm{~mm} / \mathrm{s}, \dot{\gamma}=1 \mathrm{~s}^{-1}, L=50 \mu \mathrm{m}, k=1.3 \times 10^{7} \mathrm{~m}^{-1}$, $\theta=5 \mathrm{deg}$ and $\phi=0 \mathrm{deg}, \tau_{v}=0.07 \mathrm{~s}$ and $\tau_{\dot{\gamma}}=0.04 \mathrm{~s}$. Typically, an (inexpensive) high speed scientific grade camera has a frame rate of the order of hundreds to thousands of frames per second. It therefore seems quite possible that the characteristic coherence time could be determined by statistically analysing time resolved images of the dynamic speckle pattern measured at a sufficiently high frame rate. Furthermore, because $\tau_{\dot{\gamma}}$ shows a dependence on spatial location in the image (through its dependence on $\theta$ and $\phi$ ) whereas $\tau_{v}$ does not, it might be possible to determine $\tau_{v}$ and $\tau_{\dot{\gamma}}$ separately. Scattering from large particles is most intense at small angles - this could be expected to help compensate for a camera's low sensitivity.

It was the Author's original intention to demonstrate that flow (both local strain rate and/or velocity) and optical anisotropy could be measured simultaneously by imaging both the unscattered and scattered light produced from a sheared wormlike micelles solutions at small angles. Two significant problems were encountered. Firstly, a home-made miniature cylindrical-Couette geometry carefully engineered to slip into the Malvern Autosizer and to operate immersed in a water bath leaked, making measurements unreliable. Secondly, it became clear that the statistics of the speckle pattern, even for simple fluids, were quite complicated, motivating the more detailed study of the statistical properties of dynamic speckle given in this chapter.

While the relationship between the space-time statistics of the dynamic speckle pattern, motion of the scattering centres and scattering volume is understood for a number of specific configurations, the general relationship has not been described in the literature. In order to explore this more general relation, an approach based on simulation was developed. Here the dynamic speckle pattern is simulated directly for the motion and the optical configuration of interest and space-time intensity correlation is calculated from the simulated speckle. The validity of the approach is confirmed by comparison to both analytic and measured statistics. The 'brute force' approach demonstrated here is conceptually simple and appears to be quite general. 


\subsection{Statistical properties of dynamic speckle}

Commonly, in PCS, an intensity $I(\mathbf{X}, t)$ at position $\mathbf{X}$ is monitored in time $t$. These measurements are used to develop an approximation to the normalised time (if a single detector is used) or space-time (if multiple detectors are used) intensity correlation function

$$
\begin{aligned}
g_{2}\left(\mathbf{X}_{1}, \mathbf{X}_{2}, t_{1}, t_{2}\right) & =\frac{\left\langle I\left(\mathbf{X}_{1}, t_{1}\right) I\left(\mathbf{X}_{2}, t_{2}\right)\right\rangle}{\left\langle I\left(\mathbf{X}_{1}, t_{1}\right)\right\rangle\left\langle I\left(\mathbf{X}_{2}, t_{2}\right)\right\rangle} \\
& =1+\frac{\left|\left\langle E\left(\mathbf{X}_{1}, t_{1}\right) E^{*}\left(\mathbf{X}_{2}, t_{2}\right)\right\rangle\right|^{2}}{\left\langle E\left(\mathbf{X}_{1}, t_{1}\right) E^{*}\left(\mathbf{X}_{1}, t_{1}\right)\right\rangle\left\langle E\left(\mathbf{X}_{2}, t_{2}\right) E^{*}\left(\mathbf{X}_{2}, t_{2}\right)\right\rangle} \\
& =1+\left|g_{1}\left(\mathbf{X}_{1}, \mathbf{X}_{2}, t_{1}, t_{2}\right)\right|^{2}
\end{aligned}
$$

where $E(\mathbf{X}, t)$ is the field associated with intensity $I(\mathbf{X}, t)$ and $g_{1}\left(\mathbf{X}_{1}, \mathbf{X}_{2}, t_{1}, t_{2}\right)$ is the normalised space-time field correlation function. If $\mathbf{X}_{2}=\mathbf{X}_{1}$, Eqn. 9.3 describes the normalised time intensity autocorrelation function.

From Chapter 4 , the field $E(\mathbf{X}, t)$ at a distant observation point $\mathbf{X}=X \hat{\mathbf{i}}+Y \hat{\mathbf{j}}+Z \hat{\mathbf{k}}$ due to an isotropic scattering centre located at $\mathbf{x}=x \hat{\mathbf{i}}+y \hat{\mathbf{j}}+z \hat{\mathbf{k}}$

$$
E(\mathbf{X}, t) \propto E_{0}(\mathbf{x}, t) \frac{\exp [i k r(t)]}{i k r(t)} .
$$

$E_{0}(\mathbf{x}, t)$ is the incident field and $r(t)=\sqrt{(x(t)-X)^{2}+(y(t)-Y)^{2}+(z(t)-Z)^{2}}$ is the distance between the source and the detection point. For an ensemble of $N$ such scattering centres

$$
E_{N}(\mathbf{X}, t) \propto \sum_{n=1}^{N} E_{0}\left(\mathbf{x}_{n}, t\right) \frac{\exp \left[i k r_{n}(t)\right]}{i k r_{n}(t)}
$$

where $r_{n}(t)=\sqrt{\left(x_{n}(t)-X\right)^{2}+\left(y_{n}(t)-Y\right)^{2}+\left(z_{n}(t)-Z\right)^{2}}$ and the intensity at the detection point

$$
I_{N}(\mathbf{X}, t) \propto E_{N}(\mathbf{X}, t) E_{N}^{*}(\mathbf{X}, t) .
$$

The space-time variation in the position of the scattering centres introduces a spacetime variation into the intensity at the observation point. A space-time correlation can be calculated numerically by cross-correlating (denoted $\star$ ) and averaging the measured intensities

$$
g_{2}\left(\mathbf{X}_{1}, \mathbf{X}_{2}, t_{1}, t_{2}\right) \approx \frac{I_{N}\left(\mathbf{X}_{1}, t_{1}\right) \star I_{N}\left(\mathbf{X}_{2}, t_{2}\right)}{\overline{I_{N}\left(\mathbf{X}_{1}, t_{1}\right)}} \overline{I_{n}\left(\mathbf{X}_{2}, t_{2}\right)}
$$

Yoshimura studied a related problem in [143] 144]. Here the space-time correlation functions for translation or rotation of a two-dimensional deep random phase screen through a Gaussian laser beam was considered. The random phase screen randomises the 

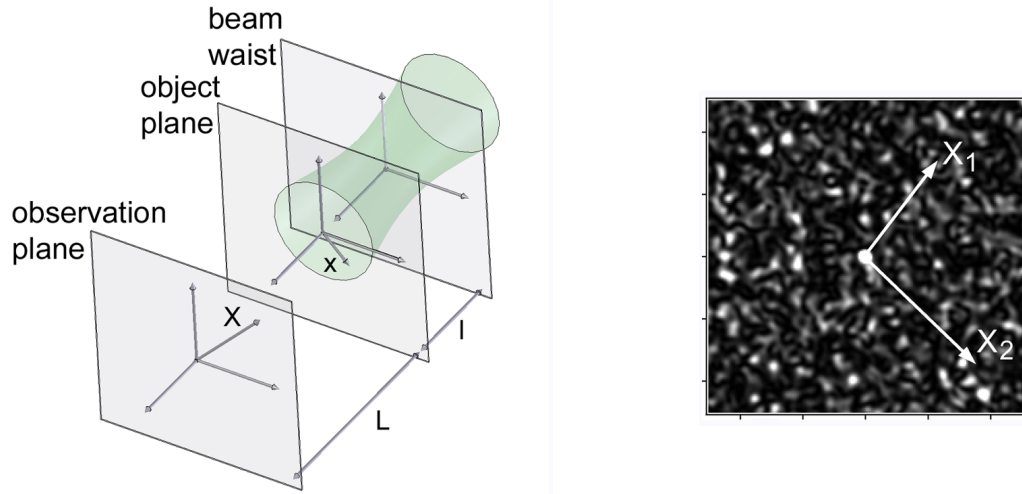

Figure 9.1: i) Free space geometry and ii) position vectors at the observation plane.

phase of the incident light. It can be considered deep if the phase is randomised uniformly over an interval $[0,2 \pi]$. Yoshimura's free-space geometry is illustrated in Fig. 9.1. The random phase screen is located at a distance $l$ from the beam waist and contains the position vector $\mathbf{x}$. The observation plane is a distance $L$ from the random phase screen and contains the position vector $\mathbf{X}$. Incident illumination $E_{0}(\mathbf{x}, t)$

$$
E_{0}(\mathbf{x}, t)=\frac{w_{0}}{w} \exp \left[\frac{|\mathbf{x}|^{2}}{w^{2}}-i\left(\omega_{0} t-k l-\frac{\pi}{\lambda \rho}|\mathbf{x}|^{2}-\phi_{0}\right)\right]
$$

was assumed. Here $w_{0}$ is the radius of the beam at the waist, $w=w_{0}\left[1+\left(\frac{l}{a}\right)^{2}\right]^{1 / 2}$ is the radius of the beam at the object plane, $\rho=l\left[1+\left(\frac{a}{l}\right)^{2}\right]$ is a measure of the curvature of the wavefront and $a=\frac{\pi}{\lambda} w_{0}^{2}$.

The space-time (magnitude-squared) field correlation function at the observation plane due to Gaussian illumination of a random phase screen undergoing uniform translation at velocity $\mathbf{v}$ in the object plane for the free-space geometry was found to be

$$
\left|g_{1}(\mathbf{R}, \tau)\right|^{2}=\exp \left[-\frac{|\mathbf{R}|^{2}}{R_{s}^{2}}+\frac{\tau_{d}^{2}}{\tau_{c}^{2}}\right] \exp \left[-\frac{1}{\tau_{c}^{2}}\left(\tau-\tau_{d}\right)^{2}\right]
$$

where

$$
\begin{aligned}
& \frac{1}{\tau_{c}}=|\mathbf{v}|\left[\frac{1}{R_{s}^{2}}\left(1+\frac{L}{\rho}\right)+\frac{1}{w^{2}}\right]^{1 / 2} \\
& \tau_{d}=\frac{\tau_{c}^{2}}{R_{s}^{2}}\left(1+\frac{L}{\rho}\right) \mathbf{v} \cdot \mathbf{R} .
\end{aligned}
$$

Here $\mathbf{R}=\mathbf{X}_{2}-\mathbf{X}_{1}$ is a displacement vector, $\tau=t_{2}-t_{1}=\Delta t$ is a delay and $R_{s}=2 l / k w$ is the characteristic speckle size. $\tau_{c}$ is a characteristic coherence time of the speckle and is proportional to the magnitude of the velocity of the phase screen. $\tau_{d}$ is a characteristic 

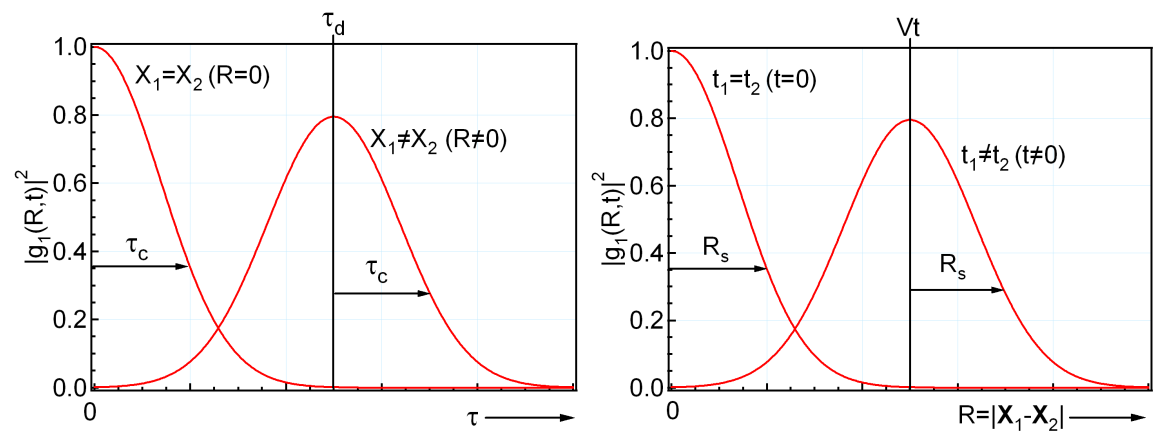

Figure 9.2: Model space-time auto $\left(\mathbf{X}_{1}=\mathbf{X}_{2}\right.$ or $\left.t_{1}=t_{2}\right)$ and cross-correlation $\left(\mathbf{X}_{1} \neq \mathbf{X}_{2}\right.$ or $t_{1} \neq t_{2}$ ) for a free space geometry according to Yoshimura's model.

'displacement' time - this quantity is proportional to a projection of the velocity of the phase screen onto the displacement vector $\mathbf{R}$. This quantity can be positive or negative and informs on the direction of motion. Equivalently Eqn. 9.9 can be rewritten

$$
\left|g_{1}(\mathbf{R}, \tau)\right|^{2}=\exp \left[-\frac{R_{s}^{2}-\tau_{c}^{2}|\mathbf{V}|^{2}}{\tau_{c}^{2} R_{s}^{2}} \tau^{2}\right] \exp \left[-\frac{1}{R_{s}{ }^{2}}|\mathbf{R}-\mathbf{V} \tau|^{2}\right],
$$

where $\mathbf{V}$ is the speckle velocity at the observation plane

The space-time correlations described by Eqn. 9.9 and 9.12 are shown schematically in Fig. $9.2 \mathrm{i}$ and ii. Both equations predict that the space-time correlation has the form of a shifted Gaussian function with a width equal to the characteristic coherence time (Eqn. 9.9) or speckle size (Eqn. 9.12) and a shift that depends on the speckle velocity at the observation screen. The time lag $\tau_{d}$ in Eqn. 9.9 is proportional to $\mathbf{v} \cdot \mathbf{R}$ - reversing the direction of flow will reverse the sign of the lag term. $\tau_{d}$ is also proportional to $1+L / \rho=1+L\left(l\left[1+\left(\frac{a}{l}\right)^{2}\right]\right)^{-1}$. Because $1+L / \rho$ depends on $l$ and the displacement $l$ can be positive or negative, the sign of $\tau_{d}$ also depends on the location of the object plane. Note that this change in sign occurs at $L / \rho=-1$ and not at the beam waist $(L=0)$ as might be assumed. For example, if $w_{0}=65 \mu \mathrm{m}, \lambda=(532 / 1.33) \mathrm{nm}$ and $L=0.1 \mathrm{~m}$ the zero crossing is offset from the waist by $13 \mathrm{~mm}$.

The statistical behaviour of the speckle pattern predicted by Eqn. 9.6 along with the method of analysis is compared to that described by Yoshimura in Fig. 9.3. The field, due to 200 scattering centres randomly distributed on an object plane at $z=l=0$ over a $200 \times 200 \mu \mathrm{m}$ area and illuminated by a Gaussian beam with waist $w_{0}=65 \mu \mathrm{m}$ at an observation plane $Z=l+L=100 \mathrm{~mm}$, is considered. The scattering centres were assumed to translate at a velocity $\mathbf{v}=5 \mu \mathrm{m} / \mathrm{s} \hat{\mathbf{i}}$. Fig. 9.3 i shows the simulated intensity variation at an instant at the observation plane. The time dependence of the speckle pattern intensity was investigated over the lines shown in Fig. 9.3 i. Fig. 9.3 ii shows the typical time variation at $Y=2 \mathrm{~mm}$. Time variation is again shown in Fig. 9.3 iii for the line indicated in 9.3 ii. These simulated intensities are cross-correlated in Fig. 9.3 iv 
to give an unnormalised space-time intensity correlation function. Based on Yoshimura's description of a deep random phase screen, it could be expected that the unnormalised space-time intensity correlation function would have the form

$$
I_{1}\left(\mathbf{X}_{1}, t_{1}\right) \star I_{2}\left(\mathbf{X}_{2}, t_{2}\right)=A \exp \left[-\frac{1}{\tau_{c}^{2}}\left(\Delta t-\tau_{d}\right)^{2}\right]+b .
$$

The simulated space-time intensity correlations functions were fitted to determine the dependence of $A, \tau_{c}$ and $\tau_{d}$ on $\Delta X=X_{2}-X_{1}$ for $X_{1}=-500 \mu \mathrm{m}, X_{1}=-167 \mu \mathrm{m}$, $X_{1}=+266 \mu \mathrm{m}$ and $Y=1,2,5,10 \mathrm{~mm}$. Fitted parameters are shown in Fig. 9.3 v-vii along with dependence calculated according to Eqn. 9.9 9.11. Note that the three $X_{1}$ values give three estimates of $A, \tau_{c}$ and $\tau_{d}$ at each $Y$ position. These estimates are not distinguished in Fig. 9.3 v-vii. The two are in close agreement suggesting that speckle pattern produced by a random distribution of isotropic scattering centres over a twodimensional scattering volume is statistically equivalent to that produced by a random phase screen. Yoshimura's analysis is restricted to two-dimensional 'scattering volumes'. An approach based on simulation does not suffer from this limitation.

\subsection{Experimental}

Laser speckle was imaged using a digital camera (Pixelink, B742F). The resolution of this camera is $1240 \times 1024$ pixels over a detector area of $8.7 \times 6.9 \mathrm{~mm}$. To minimise file size and to maximise frame readout rate, the region of interest on the detector was limited to a strip with dimensions $1280 \times 250-380$ pixels. Intensity was binned on the camera and recorded with 8 bit depth. The effective pixel pitch with the $2 \times 2$ pixel binning used was $13.4 \mu \mathrm{m}$. Speckle was imaged with fixed exposure times of the order of a few milliseconds at frame read out rates of 50 or 200 frames per second. Between $8-12 \mathrm{k}$ images were acquired in each experimental trial.

Two light sources were used: a $500 \mathrm{~mW} \lambda=532 \mathrm{~nm} \mathrm{Nd:Yag} \mathrm{laser} \mathrm{(JDS} \mathrm{Uniphase)}$ and a $15 \mathrm{~mW} \lambda=632 \mathrm{~nm}$ HeNe laser (Melles-Griot). The waist dimensions of both lasers were measured using the knife-edge method. With focusing, the waists of the Nd:Yag and HeNe lasers were $w_{0}=65 \mu \mathrm{m}$ and $w_{0}=45 \mu \mathrm{m}$. The beam was attenuated as necessary with a variable attenuator (Edmund Optics).

The statistical properties of dynamic speckle were measured in two experimental geometries: 1) translation and 2) simple shear flow. These two geometries were used to induce velocity fields of the form $\mathbf{v}=v_{0} \hat{\mathbf{i}}$ and $\mathbf{v}=v_{0} z \hat{\mathbf{i}}$ in the scattering volume.

In the translation geometry, light from the focused Nd:Yag laser was directed through a 10, 5, 2 or $1 \mathrm{~mm}$ path length cuvette containing a 12-14 wt.\% aqueous 95K PVA solution with viscosity $\eta=2-3$ Pa.S doped with monodisperse 1025, 535, 356, or $202 \mathrm{~nm}$ diameter latex spheres (Polyscience Inc.) at concentrations in the range $5 \times 10^{-6}$ to $5 \times 10^{-5} \mathrm{wt} . \%$. PVA was used to suppress diffusion and sedimentation of the spheres and the lower concentrations of latex spheres were used for longer path lengths to avoid multiple scattering. 

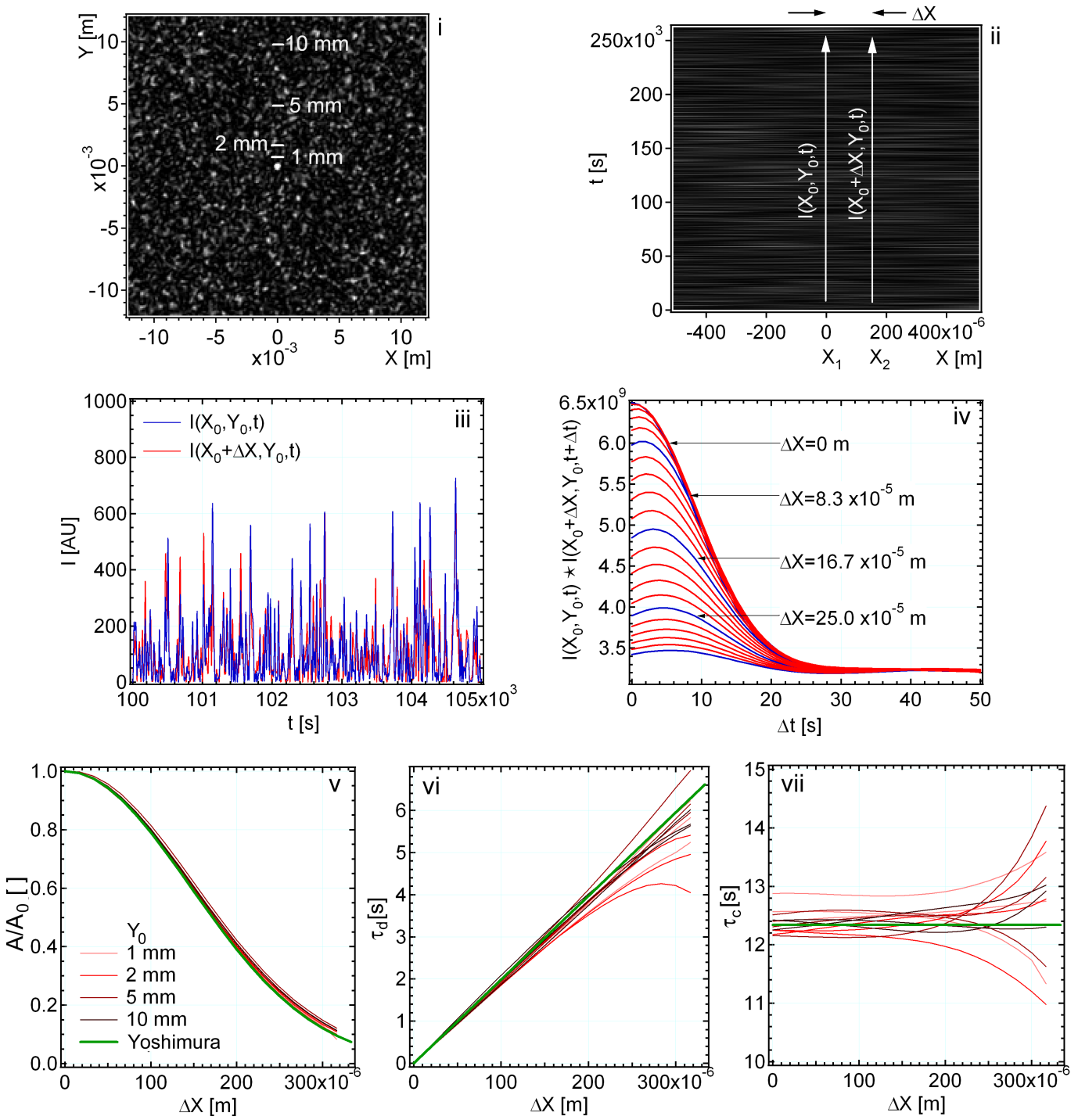

Figure 9.3: Simulated space-time correlation for a random ensemble of scattering centres on a plane that translates through a Gaussian beam with a comparison to Yoshimura's result for the translation of a deep random phase screen through a Gaussian beam (see text). 

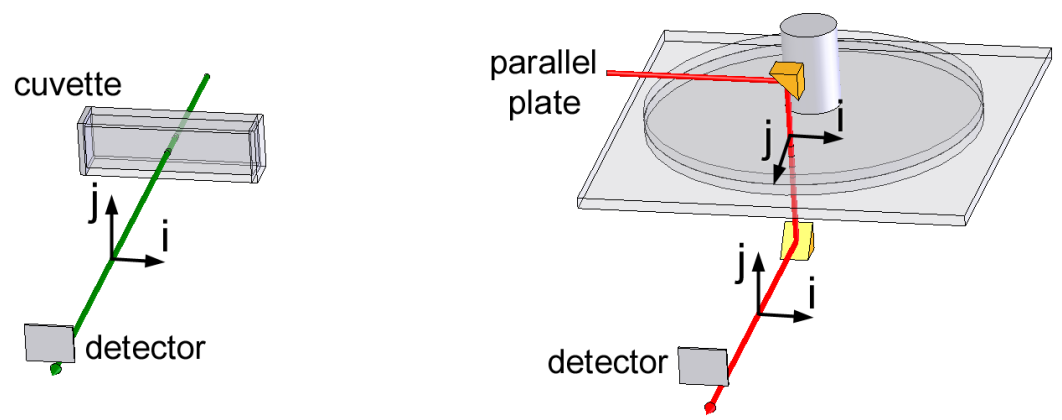

Figure 9.4: Experimental set-up for i) translation and ii) simple shear flow measurements.

The cuvettes were mounted on a motorised translation stage that moved the cuvettes through the beam at $v_{0}=0.5 \mathrm{~mm} / \mathrm{s}$. Both the motorised translation stage and camera were mounted on a labjack which fixed the detector-cuvette separation at $L=100 \mathrm{~mm}$ (as measured from the centre of the cuvette). Scattered light was detected at a horizontal 8.7 $\times 1.5 \mathrm{~mm}$ strip of interest located $y=7 \mathrm{~mm}$ above the beam. The labjack was mounted on $1 \mathrm{~mm}$ square graph paper which allowed for accurate repositioning. Measurements were made with the centre of the cuvettes at $z=l=-40,20,0,+20,+40 \mathrm{~mm}$ with cameracuvette-labjack assembly displaced $X=-6,0,+6,+12 \mathrm{~mm}$ effectively increasing the width of the strip of interest from $8.7 \mathrm{~mm}$ to approximately $24 \mathrm{~mm}$. Images were acquired at 200 frames per second.

In the simple shear flow geometry, light from the focused HeNe laser was directed into an aqueous PVA solution doped with monodisperse $1025 \mathrm{~nm}$ diameter latex spheres (Polyscience Inc.) at $5 \times 10^{-5}$ wt.\% trapped between a rheometer (Thermal Analysis AR2000) mounted $60 \mathrm{~mm}$ diameter rotating glass disc and a stationary parallel glass plate. The disc-plate separation was fixed at 2, 1.5 or $1 \mathrm{~mm}$. The beam's waist was located (approximately) at the centre of the gap. The disc was rotated at $3.14 \times 10^{-3}$ $\mathrm{rad} / \mathrm{s}$ and light directed though the fluid $17 \mathrm{~mm}$ along the $\hat{\mathbf{k}}$ axis $17 \mathrm{~mm}$ from the axis of rotation inducing a velocity $\mathbf{v}=0.053 z \hat{\mathbf{i}} \mathrm{mm} / \mathrm{s}$ in the illuminated region. Scattered light was detected at approximately $L=80 \mathrm{~mm}$ from the waist over a horizontal strip $8.7 \times 3$ $\mathrm{mm}$ of interest located approximately $y=10 \mathrm{~mm}$ above the beam. Images were acquired at 50 frames per second.

\subsection{Measurements and analysis}

\subsubsection{Translation}

The effect of increasing the width $d$ of the scattering volume on the simulated speckle pattern is illustrated Fig. 9.5. The speckle pattern is calculated for 200 scattering centres 

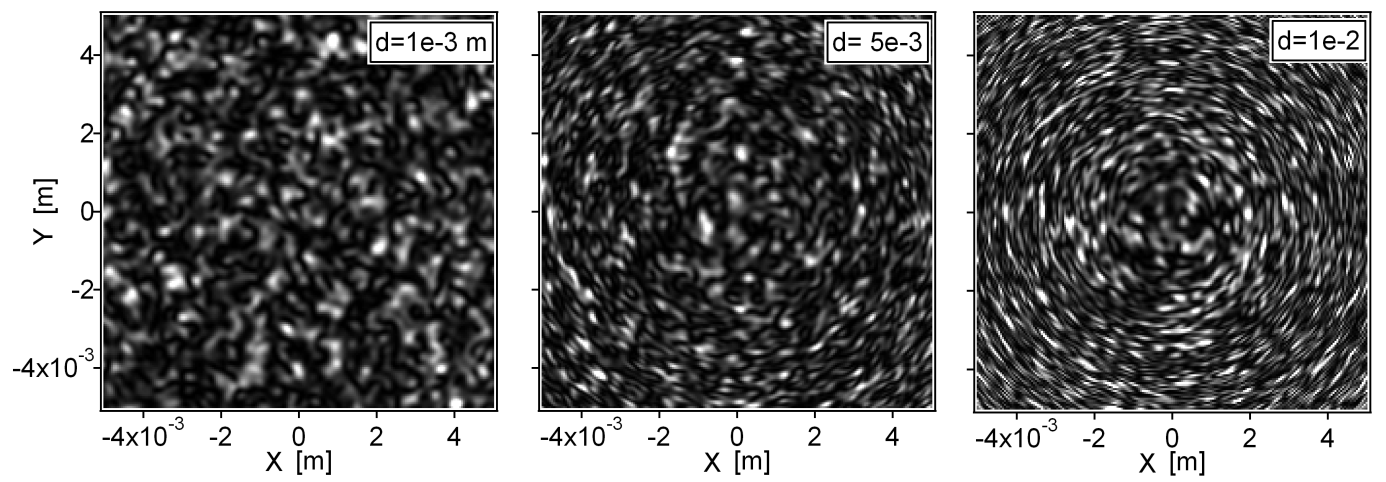

Figure 9.5: Simulated speckle patterns with increasing path length $d$ of the scattering volume.

randomly distributed through an object volume centered on $l=0$ over an $200 \mu \mathrm{m} \times 200 \mu \mathrm{m}$ $\times d$ volume and assuming $L=100 \mathrm{~mm}, w_{0}=65 \mu \mathrm{m}$. As the length of the scattering volume is increased the speckle shows increasing 'barrel' distortion. In Yoshimura's analysis, the characteristic size of the speckle $R_{s}$ is constant - clearly this is no longer the case when the length of the scattering volume becomes significant. In Fig. 9.6 the simulated speckle is compared to measured speckle for $1025 \mathrm{~nm}$ diameter latex spheres in $2 \mathrm{~mm}$ and $10 \mathrm{~mm}$ path length cuvettes located at $l=0$. The simulation reproduces the 'barrel' distortion observed in the measurement.

The measured speckle pattern was affected by two artefacts: interference fringes and motor jitter. Both static and moving fringes were observed as the cuvette was translated through the beam. These fringes are visible in measurements shown in Fig. 9.6. The translation stage was driven by a stepping motor. Every 2-5 frames the speckle pattern was observed to jump coherently by a few pixels.

After acquisition the intensity data were filtered and correlations calculated. The characteristic coherence time $\tau_{c}$ was determined by autocorrelating the intensity in time and fitting to Eqn. 9.13. The characteristic displacement time $\tau_{d x}$ and $\tau_{d y}$ were calculated by cross-correlating the intensity at adjacent pixels in either the $\hat{\mathbf{i}}$ or $\hat{\mathbf{j}}$ directions and fitting the correlation to Eqn. 9.13 .

Filtering was used to reduce the effect of the artefacts discussed above on the resulting correlation. The intensity data were filtered in space by convolution with a 4.8 (binned) pixel width Gaussian function and/or in time with a 7 frame running average filter prior to correlation. This filtering smears the speckle in both space and time. This space and time filtering was used in the calculation of $\tau_{c}$. Only time filtering was used in the calculation of $\tau_{d x}$ and $\tau_{d y}$.

The measured characteristic times $\tau_{c}, \tau_{d x}$ and $\tau_{d y}$ are shown in Fig. 9.7. As the cuvette's width increases, the statistics show significantly more spatial variation. The 


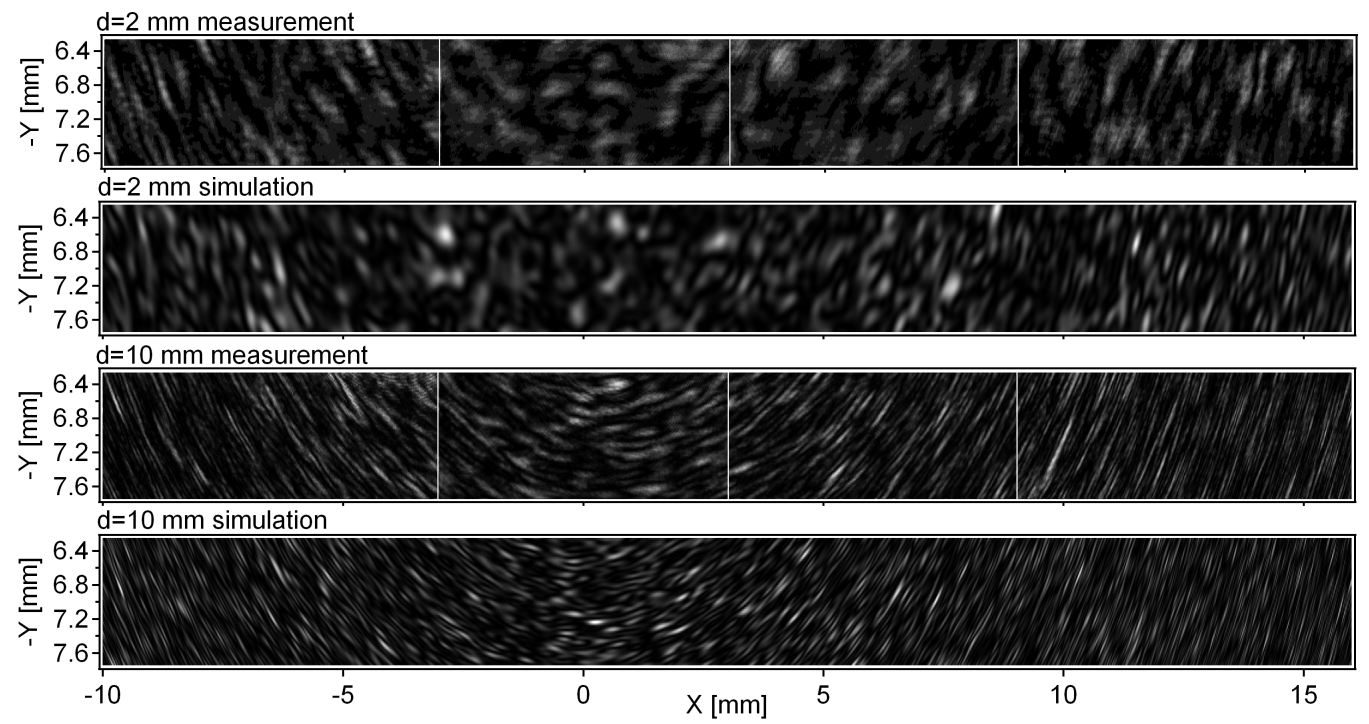

Figure 9.6: Measured and simulated speckle patterns for $d=2$ or $10 \mathrm{~mm}$ path length cuvettes.

characteristic time $\tau_{d x}$ and $\tau_{d y}$ reflect coherent motion of the speckle pattern. For the narrower scattering volume the speckles appear to move in one direction - $\tau_{d x}$ is constant and $\tau_{d y}$ is close to zero as is predicted by Yoshimura's model. For the wider scattering volumes they appear to 'circulate' about the beam. The direction of motion/circulation is dependent on the location of the cuvette in the beam with $\tau_{d x}$ being positive in some cases and negative in others. This change in direction is predicted by Yoshimura's model - the quantity $1+L / \rho$ in Eqn. 9.11 can be positive or negative. Note that this change in sign does not occur when the cuvette is centred on $z=l=0$.

The speckle pattern was simulated for $50 \mathrm{k}$ time steps for a $17 \times 17$ pixel area centred on $Y=7 \mathrm{~mm}$ and $X=-15,-14,-13 \ldots 13,14,15 \mathrm{~mm}$. The resulting images were postprocessed (filtered) as above and characteristic times calculated by fitting the auto- and cross-correlations and are shown in Fig. 9.7. Two sets of $\tau_{c}$ data are shown. The blue data has identical post-processing to that used in the measured data. The simulated $\tau_{c}$ is quite comparable in structure to the measured $\tau_{c}$ albeit with a slight negative offset. Motor jitter could be expected to increase the width of the time filtered speckles. As indicated by Eqn. 9.10 the coherence time is dependent on $R_{s}$. To include the broadening influence of the motor jitter on $\tau_{c}$ the simulated data were spatially filtered by convolving with a 1.7 (binned) pixel width Gaussian function before spatially and temporally filtering and as before. Fitted $\tau_{c}$ with spatial filtering is shown in red. Spatial filtering essentially offsets the simulated $\tau_{c}$ value. The post-processing used for simulated $\tau_{d x}$ and $\tau_{d y}$ is identical to that used for the measured data. The measured and simulated statistics are very comparable. 

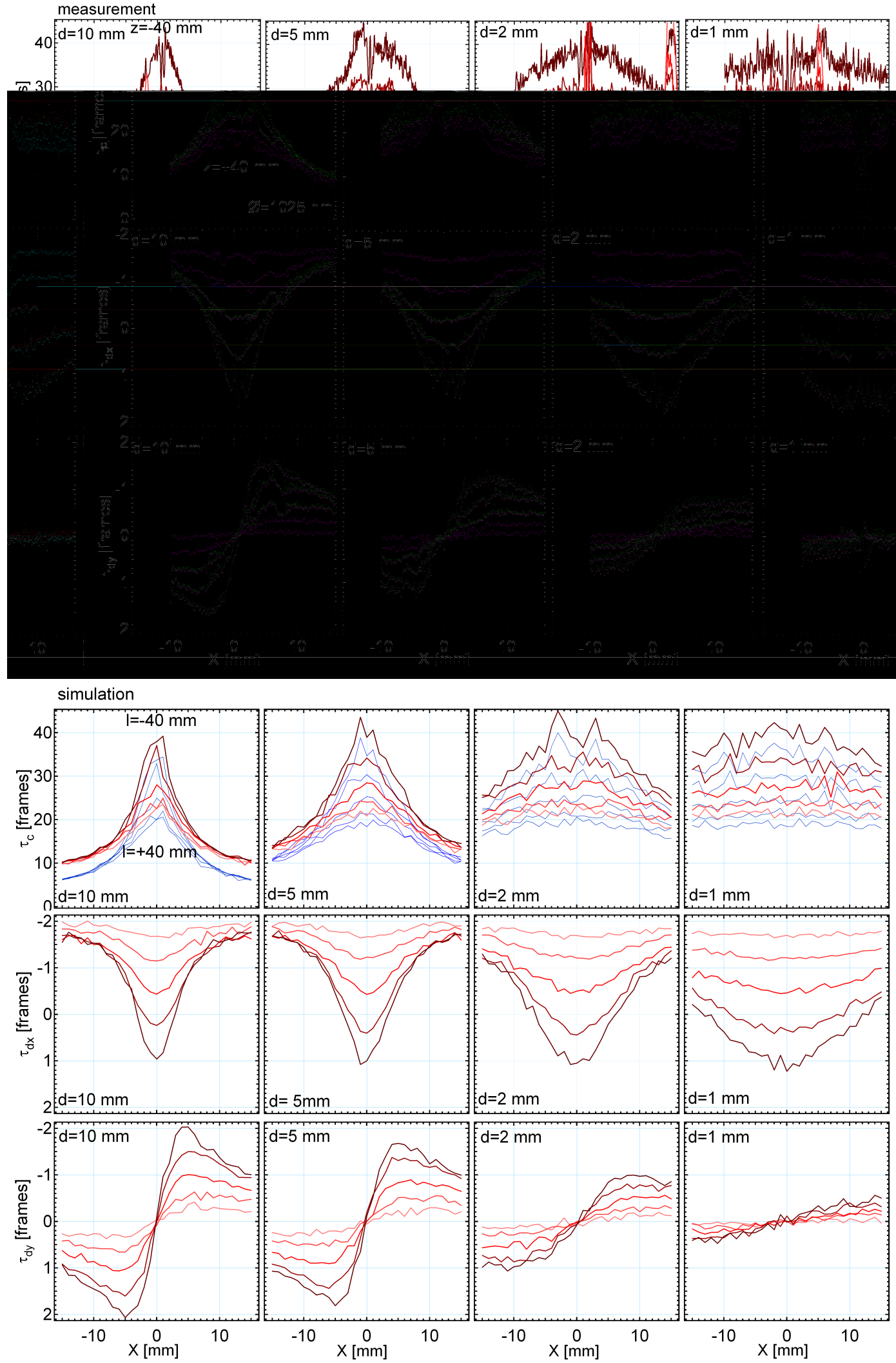

Figure 9.7: Measured and simulated characteristic times for the translation geometry (see text). 

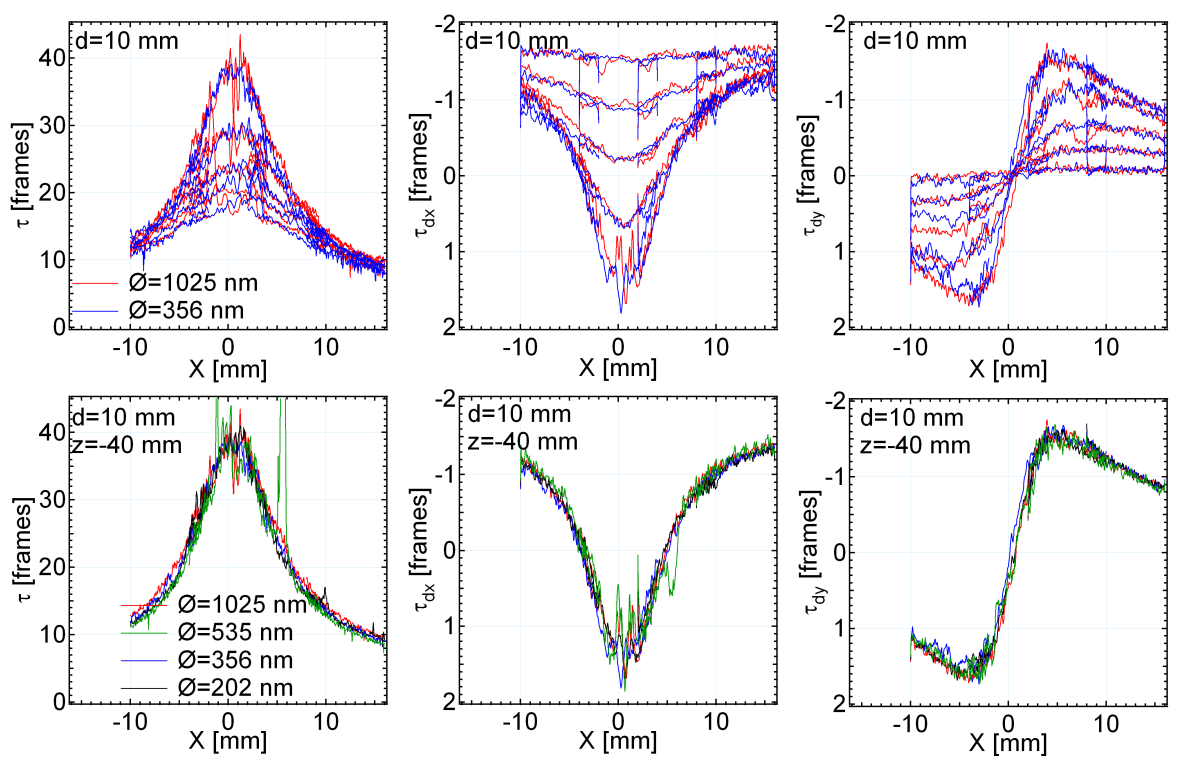

Figure 9.8: Measured characteristic times for suspensions of latex spheres of different sizes.

In the simulation, it was assumed that angle dependence $\theta$ of the scattering amplitude $S(\theta)$ could be neglected. As a check, a subset of the measurements above were repeated with 535, 356 or $202 \mathrm{~nm}$ diameter latex spheres. Characteristic times are shown in Fig. 9.8. The measured characteristic times for 535, 356 or $202 \mathrm{~nm}$ diameter spheres map onto the $1025 \mathrm{~nm}$ diameter measurements indicating that it is not unreasonable to neglect angle dependence of the scattering amplitudes at small angles.

\subsubsection{Simple shear flow}

Static images of measurements made in the parallel-plate configuration are shown in Fig. 9.9. Note that $L$ was revised from an approximately measured $L=80 \mathrm{~mm}$ to $L=90 \mathrm{~mm}$ on the basis of this comparison. Motor jitter artefacts were not observed due to the smooth rotation of the disc by the rheometer; however, interference artefacts were.

As before, $\tau_{c}$ was evaluated by fitting the intensity autocorrelation to Eqn. 9.13 after filtering in space by convolving the data with a 5.6 (binned) pixel width Gaussian function, it was filtered in time with a 3 frame running average. The $\tau_{d x}$ and $\tau_{d y}$ displacement times were evaluated by fitting the cross-correlation between adjacent pixels after filtering in time with a 3 point running average. Fitted $\tau_{c}, \tau_{d x}$ and $\tau_{d y}$ over the region of interest on the detector are shown in Fig. 9.10 i-iii. Line profiles averaged between the lines shown in Fig. 9.10 i-iii are reported in Fig. 9.10 iv-vi. The simulated characteristic times for $20 \mathrm{~K}$ time steps are shown below. There are several notable differences between the measured and simulated characteristic times. The central peak is split in the simulation whereas 


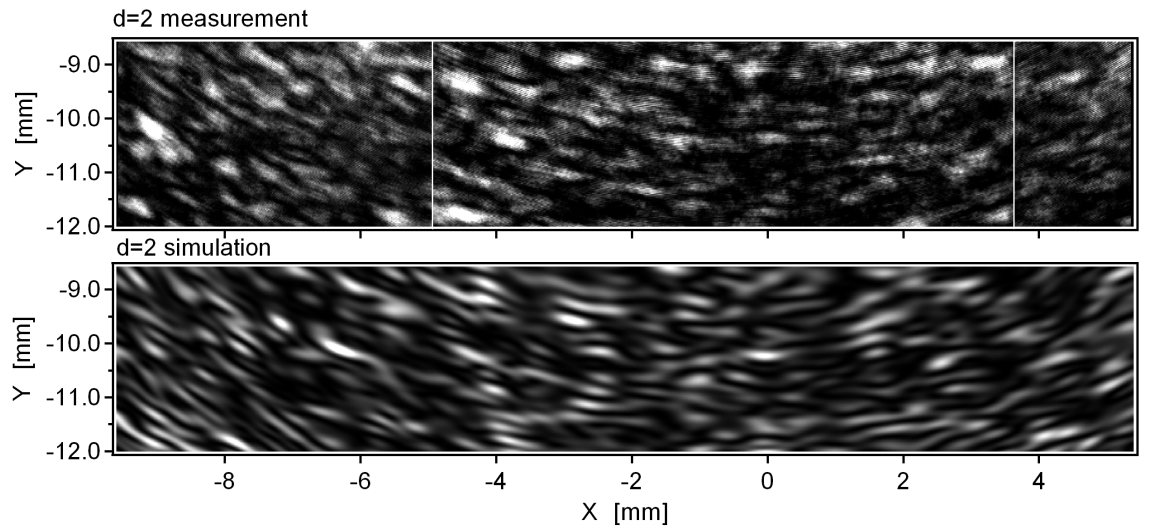

Figure 9.9: A comparison between measured and simulated speckle patterns observed for a path length of $d=1.5$ in the simple shear flow geometry.

splitting is not observed in the measurements. A decrease was observed between $\mathrm{X}=-2$ to $+2 \mathrm{~mm}$ in the measured $\tau_{d x}$ which is not seen in the simulation. Outside this $X$ range the simulation reproduces the measured behaviour quite well.

\subsection{Discussion}

It has been demonstrated here that the space-time statistics of a dynamic speckle pattern can readily be simulated. In principle, this information can be used to understand flow behaviour of the scattering centres through the scattering volume.

The simulation discussed here is time intensive. It takes about $20 \mathrm{~s}$ on a $2 \mathrm{GHz} \mathrm{PC}$ to evaluate the intensity variation for 200 scattering centres at $20 \mathrm{k}$ time steps for a single point at the observation plane. The simulation described in the experimental section took about one month on $62 \mathrm{GHz}$ processors.

The frame rate of the camera sets a lower limit on the coherence time that can be measured. The frame rate is limited by: 1) exposure time - this depends on the intensity of the scattering; 2) the time required to transfer the image to a camera's frame buffer - this depends on the dimensions of the image and 3) the time required to transfer the image from the frame buffer to the host PC - this depends on the file size of the image and the communication protocol used. The frame rate here was limited in most cases by 2 and 3. Ideally, the data would be pre-processed in hardware/firmware on the camera itself.

The scattering centres were considered to be isotropic and polarisation effects were neglected in the preceding analysis. These effects can readily be included using the matrix formalism discussed in Chapter 3. For anisotropic scattering centres, it would be expected 

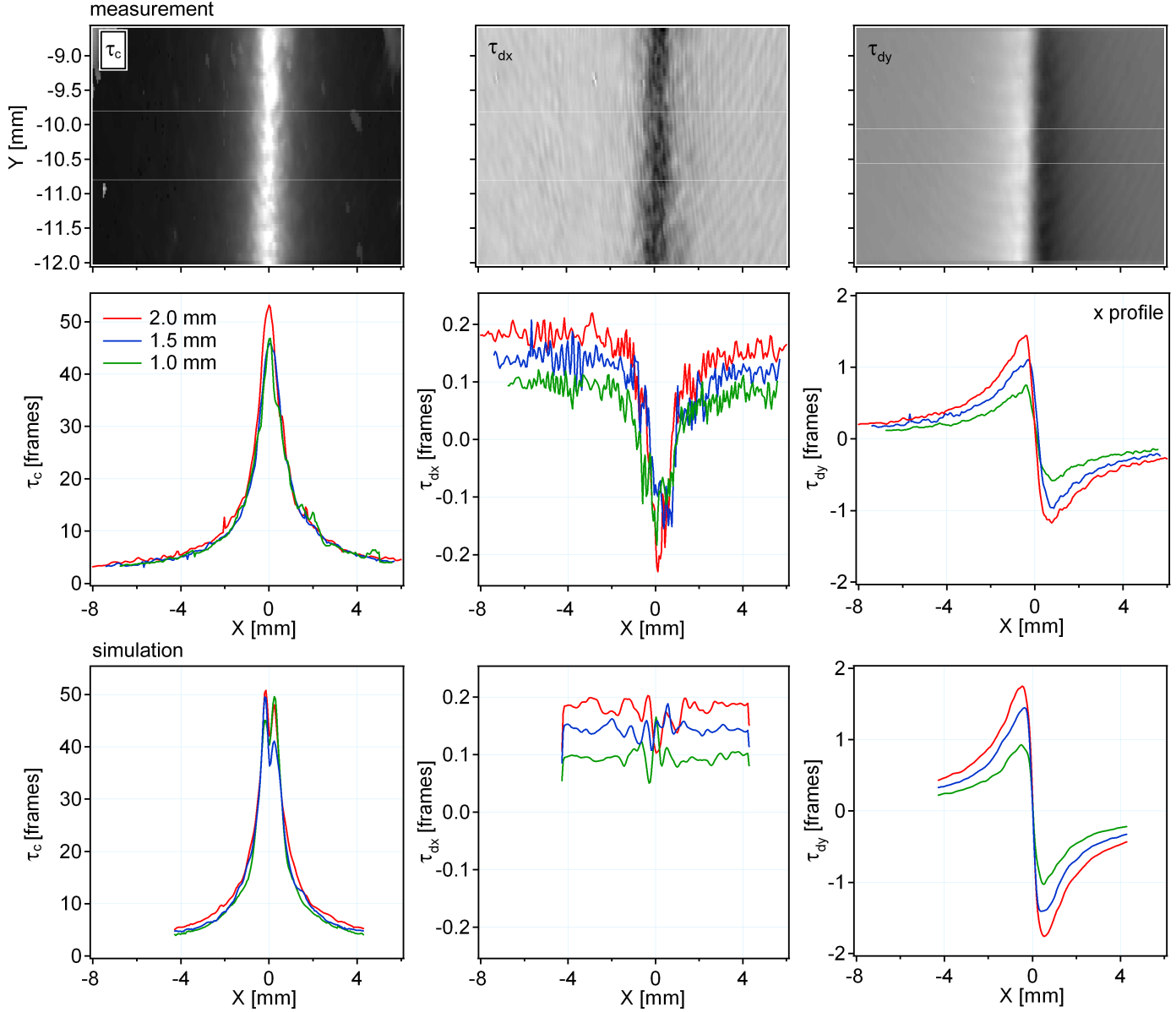

Figure 9.10: Measured and simulated characteristic times for the simple shear flow geometry (see text). 
that

$$
\mathbf{E}_{N}(\mathbf{t})=\sum_{n=1}^{N} \frac{\exp \left[k r_{n}(t)\right.}{i k r_{n}(t)}\left[\begin{array}{ll}
S_{2, n}(\theta, t) & S_{3, n}(\theta, t) \\
S_{4, n}(\theta, t) & S_{1, n}(\theta, t)
\end{array}\right]\left[\begin{array}{c}
E_{0,2}(\mathbf{x}, t) \\
E_{0,1}(\mathbf{x}, t)
\end{array}\right]
$$

It may also be possible to simulate the dynamic speckle pattern produced in DWS. The following assumptions seem reasonable:

1. Only light reradiated by 'scattering centres' located in the detection plane, a distance $\dot{\gamma}_{0} l^{*}$ from the wall and within a region of interest set by the focusing optics or an aperture directly contribute to the speckle pattern. Light radiated by other centres is scattered internally and is not detected or escapes without detection. If this assumption is correct, the characteristic speckle size is strongly dependent on the dimensions of the region of interest.

2. The absolute phase of the field illuminating a particular scattering centre in the detection plane depends on the path length $s$ of the path the diffusing light follows through the scattering medium and the phase of the incident light. This path can be simulated by computing a random walk that connects an incident source point to the location of the scattering centre within the detection plane.

3. Motion of the scattering centres distorts the simulated path, modifying the path length and phase of the field illuminating the scattering centres in the region of interest and introducing time dependence into the radiated field and speckle pattern. Translation of the scattering centres through the region of interest may produce a transit time dependence. Transit time dependence could be tested by translating a solid diffuse object (a thick piece of teflon tape, for example) through a laser at $z=l$ and examining statistics of the dynamic speckle pattern.

Together these assumptions suggest that the dynamic speckle pattern could be calculated from a field of the form

$$
E_{N}(X, t)=\sum_{n=1}^{N} E_{0, n} \exp \left[i \phi_{n}(t)\right] \frac{\exp \left[i k r_{n}(t)\right]}{i k r_{n}(t)} .
$$

Here $r_{n}$ is the distance between the 'scattering centres' in the region of interest in the detection plane of the sample and observation plane. $\exp \left[i k r_{n}(t)\right] /\left(i k r_{n}(t)\right)$ is the field radiated by these 'scattering centres'. The phase $\phi=2 \pi s(t) / \lambda$, is the time dependent phase shift introduced by multiple scattering between the source point and the detection point and could be calculated using simulation. $E_{0, n}$ is the amplitude of the field at the source point. 


\section{Chapter 10}

\section{Concluding remarks}

\subsection{Summary}

The rheological behaviour of three wormlike micelles solution systems: RHB, RHA and RHC, composed from the surfactant cetylpyridinium chloride, the counterion sodium salicylate, and the salt sodium chloride in water were studied using both conventional mechanical rheology and the rheo-optical techniques: visualisation, diffusing wave spectroscopy (DWS), ellipsometry and photo-correlation spectroscopy (PCS).

Both the linear and nonlinear rheology of the wormlike micelles solutions were examined using mechanical rheometry. Shear thinning was observed in the pseudo steady-state flow curves of all samples with a subset showing a stress plateau. A slight deviation from the Maxwell-like response predicted by the reptation-reaction model in the fast break limit was observed. This deviation appears to be common in the literature data. In accordance with the predictions of the reptation-reaction model, most samples that showed a Maxwelllike response exhibited a stress plateau with $\sigma_{c} / G_{M}=0.69 \pm 0.11$ and $\tau_{M} \dot{\gamma}_{c}=1.7 \pm 0.6$. $\sigma_{c} / G_{M}$ is very close to the theoretical value of $\sigma_{c} / G_{e}=0.67 . \tau_{M} \dot{\gamma}_{c}$ is smaller than the theoretical value of $\tau_{r} \dot{\gamma}_{c}=2.6$; this may be due to the difficulty in estimating $\dot{\gamma}_{c}$. It was noted that several RHC samples showed a Maxwell-like response without showing a stress plateau. Significant stress fluctuations, seen elsewhere, were not clearly observed in transient non-linear rheology of the RHB system indicating that this behaviour is not universal. While $\mathrm{CPCl}$ source was not found to influence the rheology of a test RHB sample strongly, the experimental geometry was shown to influence the measured rheology with significant stress fluctuations observed in 1 deg cone-plate geometry.

Shear induced turbidity was directly visualised in an optical cone-plate, parallelplate and cylindrical-Couette flow geometry. Turbid rings were observed in both the cone-plate and parallel-plate geometries in samples that showed a stress plateau. These rings were observed to migrate slowly through the fluid. Shear induced turbidity was also observed in samples that lacked a stress plateau; however, the rings were absent. Shear induced turbidity was less obvious in the cylindrical-Couette geometry possibly because 
of the illumination used. Transient rings stacked along the vorticity direction were noted at the onset of a step in strain rate exceeding the critical strain rate.

The validity of the Poisson-renewal model, which extends the reptation-reaction model to include the influence of high frequency Rouse and breathing modes on linear rheology of wormlike micelles solutions, was examined using both DWS and PCS microrheology and conventional mechanical rheology. Measurements were fitted to either a four or a five parameter Poisson-renewal model. In most cases, qualitative agreement was observed between the measured and fitted data. Fitted parameters were used to estimate the average length of the wormlike micelles studied.

In a novel application of the technique, DWS's spatial sensitivity to shear induced relative motion was used to study the flow behaviour of RHB wormlike micelles solutions in three experimental flow geometries. Measurements in the cylindrical-Couette geometry were consistent with the flow behaviour predicted on the basis of a modified Cross model and indicated that samples that exhibited a stress plateau were shear banding. The temporal stability of the measured DWS-flow correlation suggested that shear banding was quite stable. This observation is consistent with mechanical observations. Flow curves were determined from the DWS-flow correlations by fitting the data. In 3 of 4 cases the fitted flow curve was comparable to the flow curve measured mechanically. In a second approach, the strain rate profile was determined by fitting the data to behaviour predicted by a 3 parameter Bézier parameter model. The resulting strain rate profile was comparable to that predicted by the optical flow curve justifying this novel approach. Flow-DWS measurements in the cone-plate geometry were more difficult to interpret. Here it appeared that the sample might be slipping - although this interpretation is far from unambiguous. Flow-DWS correlations measured in the parallel-plate geometries were the most difficult to interpret. Here the flow appeared to show radial variation which was inconsistent with behaviour predicted according to the lever rule.

The flow behaviour of RHB wormlike micelles solutions was studied in a cylindricalCouette geometry using homodyne PCS and ellipsometry in an optical configuration that permitted simultaneous point-wise measurements of local strain rate, optical anisotropy and scattered intensity across the geometry gap. Measurements of the strain rate were complicated by the appearance of shear induced turbidity in solution. The strain rate variation observed across the geometry gap in the RHB samples lacking a stress plateau was largely consistent with the flow behaviour expected on the basis of the flow curve. Shear banding was observed in samples that exhibited a stress plateau; the partitioning observed, however, was subtler than suggested by the lever rule. It was noted that this behaviour was consistent with behaviour observed in the literature. The average stress optic coefficient of the RHB samples was measured as $C=(-1.7 \pm 0.3) \times 10^{-7} \mathrm{~Pa}^{-1}$. This is close to the stress optic coefficient $C=-2.3 \times 10^{-7} \mathrm{~Pa}^{-1}$ measured in a related system. Local optical anisotropy was found to correlate with variations in the local strain rate within the gap. In particular, bands of high and low birefringence correlated with bands of low and high strain rate in the shear banding samples. Local strain rate and optical anisotropy were also correlated with local scattered intensity suggesting that shear 
banding and shear induced turbidity are in some way connected.

The space-time statistics of the dynamic speckle pattern produced by the flow induced motion of embedded scattering centres through a laser were simulated with a view to the development of a camera-based rheo-optical technique capable of resolving flow at small angles to complement other camera-based small angle techniques. In the simulation, the speckle pattern was calculated by simply summing the field radiated by a random ensemble of point scattering centres in a Gaussian beam at a distant detection plane. The scattering centres were stepped through the beam in a way prescribed by an applied velocity field and the resulting speckle patterns were autocorrelated in time or cross-correlated in space and time to give the space-time statistics of the simulated dynamic speckle pattern. These space-time statistics were compared to the space-time statistics measured in two simple flow configurations in a number of optical set-ups. The simulated and measured statistics showed good agreement justifying the 'brute force' approach used here.

Flow-DWS was also used to probe the flow behaviour of yoghurt. The flow-DWS correlations suggested that flow behaviour of this yield-stress soft-glassy material in a cone-plate geometry was unusual. These observations were verified in a cylindrical-Couette geometry using NMR velocimetry by a collaborator. Simple partitioning into a high and low strain rate band was not observed. It was postulated that this partitioning, seen in other soft-glassy materials, was absent because of erosion due to wall slip/stick.

\subsection{Is shear banding in wormlike micelles caused by flow- concentration coupling?}

A correlation between turbidity and strain rate was observed in Chapters 5 and 8 suggesting that these two effects may be connected in some way. An increase in turbidity is seen in some sheared polymer solutions. This increase has been associated with large local variations in concentration called 'critical concentration fluctuations'. These fluctuations have been attributed to the diffusion of the polymer strands from less concentrated, less entangled, less viscous regions into more concentrated, more entangled, more viscous regions. That is, in the right conditions, the polymer strand may diffuse up its own concentration gradient. Because the structures formed in surfactant solutions are concentration dependent, shear induced critical concentration fluctuations in polymer-like wormlike micelles solutions could lead to the formation of a shear induced phase or structure. If this new phase were to form in regions of higher concentration (liquid crystals or a precipitate can form in surfactant solutions at high concentration at a fixed temperature as illustrated in Chapter 5) and if the lifetime of these structures were longer than the lifetime of the wormlike micelles then there would be a net flow of surfactant into the new phase. This would reduce the local concentration of surfactant 'in solution'. That is, the new phase temporarily sequesters the surfactant from solution. Because the dimensions of the wormlike micelles depend on concentration and because viscosity depends on the dimensions of the micelles, such a change would lead to a change in the local viscosity of the solution. 

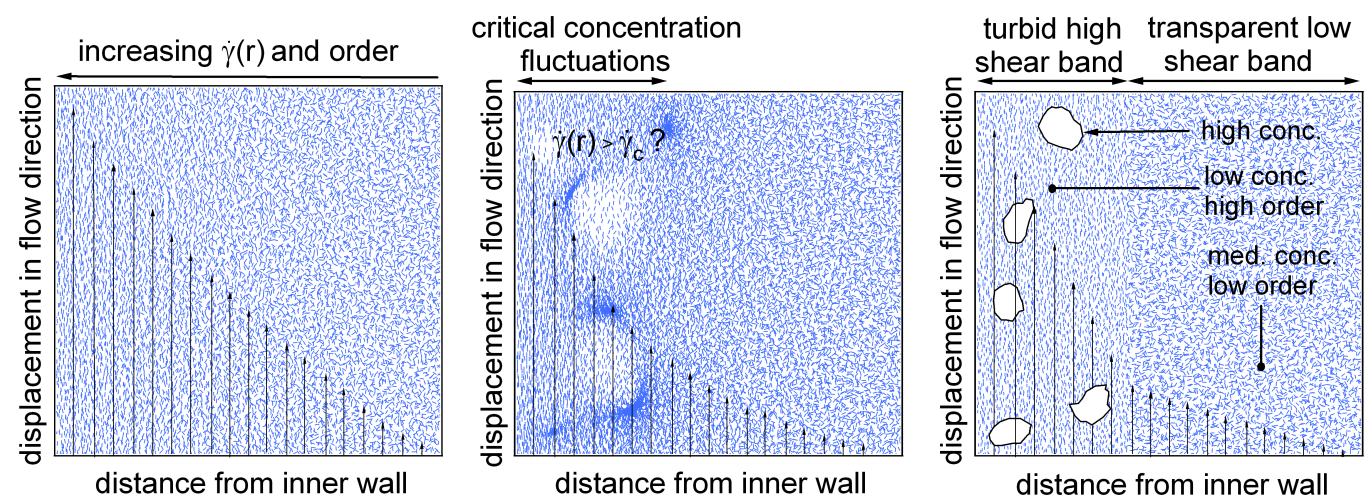

Figure 10.1: i) Because of the stress gradient across the gap of a cylindrical-Couette geometry (curvature not shown) the local strain rate decreases from the inner wall. ii) As the apparent strain rate increases towards the critical strain rate, critical concentration fluctuations may begin to occur in the region of highest local strain rate. The structures that form in surfactant solutions are concentration dependent. iii) If a new phase were to form with a lifetime greater than that of the wormlike micelles, there would be a net flow of surfactant into these structures, reducing the local concentration of surfactant in solution. Because the dimensions of the wormlike micelles are dependent on concentration, shorter wormlike micelles (for example) may form and the local viscosity of the solution may drop. This would produce a heterogeneous, low viscosity, high strain rate band and a homogeneous, high viscosity, low strain rate band as was observed.

For example, a reduction in local concentration might lead to shorter micelles and a lower local viscosity. In such cases, a turbid high strain rate band would form as was observed. Alternatively, if the reduction in concentration favoured longer micelles, the local viscosity might increase. In the cylindrical-Couette geometry the higher viscosity region would, presumably, initially form in the region of highest local strain rate at the inner wall. The formation of this low strain rate band at the inner wall would lead formation of a higher strain rate band at the outer wall. This situation would appear to be intrinsically unstable.

The appearance of turbidity in the high strain rate band indicates that this band is no longer spatially homogeneous. This observation is potentially important from an experimental perspective. Different experimental techniques probe structure on different length scales. Because structures of two quite different length scales may be present, some care must be taken with the interpretation of the data. For example, if only the 'small' wormlike micelles were observable, a shear banding wormlike micelles solution would appear to subdivide into well ordered and less well ordered regions suggesting that shear banding is caused by an isotropic to nematic phase transition. This explanation does not appear to account for the additional turbidity observed and seems at odds with the observation that the isotropic to nematic phase transition (in the RHB system) in 
equilibrium conditions occurs at concentrations many times higher than the concentrations at which shear banding is observed.

\subsection{Future work}

Soft-glassy materials show a yield stress and an associated stress plateau at low strain rates. Like some wormlike micelles solutions, soft-glassy materials have been seen to partition spatially into low and high strain rate bands in the vicinity of the stress plateau [145]. Recently, Gibaud and coworkers [146] [147] compared the flow behavior of a soft-glassy material in both a smooth and rough walled cylindrical-Couette geometry. In the rough walled geometry, the fluid spatially partitioned into a high and low strain rate band. In the smooth wall geometry, significant slip/stick was observed at the walls and the flow behaviour was much more complicated. Several continuum models predict that the intrinsic mechanical instability of the fluid will produce fluctuations in the flow behaviour. Typically, these models do not account for the effects of slip/stick at the walls on flow. A stress plateau was clearly observed in some of the RHB samples yet both fluctuation and wall slip/stick, which could be expected to depend on the surface roughness and possibly surface hydrophobicity, were not observed. Wall slip/stick was noted in measurements shown in Fig. 3.12 along with fluctuation in the flow behavior. This raises an interesting question: to what extent are fluctuations seen in some wormlike micelles solutions due to wall slip/stick and to what extent are they due to mechanical instabilities? To distinguish between effects that are produced by slip/stick and therefore do not need an explanation in terms of mechanical instability and those that are due to mechanical instabilities, the flow behaviour of identical samples could be systematically studied in smooth and rough walled geometries following the protocol discussed in [146].

As noted in Chapter 2, the underlying constitutive relation for some entangled monodisperse polymer solution/melts is expected to show a region of negative slope. To date, shear banding has not been definitively observed in entangled polymer solution/melts in steady-state flow conditions. This appears to represent a problem for a shear banding model based on a mechanical instability. Is shear banding not observed in polymer solutions because demixing with the formation of a metastable phase is less likely to occur in these materials? Near a spinodal point an entangled polymer solution will spontaneously phase separate into polymer rich and solvent rich phases. It may be possible to induce a spinodal decomposition by shearing a sample near the spinodal point. Does shear banding occur in such a sample? If so, this might add weight to the idea that some kind of qualitative change in the microstructure (like that produced by a phase transition) is required for shear banding to occur. Again this seems straightforward to test.

The flow behaviour of shear banding wormlike micelles solutions in the parallel-plate geometry appears to be particularly unusual. It would be useful to reexamine the flow behaviour using multiple flow-DWS optical configurations. These flow measurements could be complemented with measurements made using some other well established technique, such as NMR velocimetry, in order to better understand the application and limitation 
of the flow-DWS and to explore the flow behaviour of shear banding wormlike micelles in this parallel-plate geometry. 


\section{References}

[1] P.-G. de Gennes. Nobel prize acceptance speech, 1991.

[2] R. Zana and E. W. Kaler. Giant micelles: properties and applications. CRC Press, United States of America, 1 edition, 2007.

[3] F. A. Morrison. Understanding rheology. Oxford University Press, New York, 1 edition, 2001.

[4] R. G. Larson. The structure and rheology of complex fluids. Oxford University Press, Oxford, U.K., 1999.

[5] R. G. Larson. The Constitutive Equation for Polymer Melts and Solutions. Butterworths Series in Chemical Engineering. Butterworths, United States of America, 1st edition, 1988.

[6] A. R. Paterson. Dynamics under the influence of stochastic forces. Cambridge Unversity Press, United Kingdom, 1983.

[7] H. A. Barnes, J. F. Hutton, and K. Walters. An introduction to rheology. Elsevier Science, Oxford, U.K., 1989.

[8] M. R. Rubinstein and R. H. Colby. Polymer Physics. Oxford University Press, United States of America, 2003.

[9] H. J. Pain. The physics of vibrations and waves. John Wiley and Sons, Ltd., United States of America, 4 edition, 1992.

[10] P. R. Saulson. Thermal noise in mechanical circuits. Physical Review D, 42:24372445, 1990.

[11] B. J. Berne and R. Pecora. Dynamic Light Scattering - with applications to Chemistry, Biology and Physics. Dover Publications Inc., 2nd edition, 2000.

[12] G. G. Fuller. Optical Rheometry of Complex Fluids. Oxford University Press, New York, 1995.

[13] H. Rehage and H. Hoffmann. Viscoelastic surfactant solutions model systems for rheological research. Molecular Physics, 74:933-973, 1991. 
[14] M. E. Cates. Reptation of living polymer - dynamics of entangles polymers in the presence of reversible chain-scission reactions. Macromolecules, 20:2289-2296, 1987.

[15] R. Granek and M. E. Cates. Stress relaxation in living polymers - results from a poisson renewal model. Journal of Chemical Physics, 96:4758-4767, 1992.

[16] M. S. Turner and M. E. Cates. Linear viscoelasticity of living polymers - a quantitative probe of chemical relaxation times. Langmuir, 7:1590-1594, 1991.

[17] M. S. Turner and M. E. Cates. Linear viscoelasticity of wormlike micelles :A comparison of micellar reaction kinetics. Journal de Physique II, 2:503-519, 1992.

[18] M. S. Turner, C. Marques, and M. E. Cates. Dynamics of wormlike micelles - the bond interchange reaction scheme. Langmuir, 9:695-701, 1993.

[19] A. Khatory, F. Lequeux, F. Kern, and S. J. Candau. Linear and nonlinear viscoelasticity of semi-dilute solutions of wormlike micelles at high salt concentration. Langmuir, 9:1456-1464, 1993.

[20] M. E. Cates. Nonlinear viscoelasticity of wormlike micelles (and other reversibly breakable polymers). Journal of Physical Chemistry, 94:371-375, 1990.

[21] N. A. Spenley, M. E. Cates, and T. C. B. McLeish. Nonlinear rheology of wormlike micelles. Physical Review Letters, 71:939-943, 1993.

[22] V. Schmitt, C. Marques, and F. Lequeux. Shear-induced phase separation of complex fluids: The role of flow-concentration coupling. Physical Review E, 52(4):4009-4015, 1995 .

[23] T. C. B. McLeish and R. C. Ball. A molecular approach to the spurt effect in polymer melt flow. Journal of Polymer Science Part B: Polymer Physics, 24:1735-1745, 1986.

[24] R. Makhoufi, J. P. Decruppe, A. Ait-Ali, and R. Cressely. Rheo-optical study of worm-like micelles undergoing a shear banding flow. Europhysics Letters, 32:253258, 1995.

[25] E. Cappelaere, J. -F. Berret, J. P. Decruppe, R. Cressely, and P. Lindner. Rheology, birefringence and small-angle neutron scattering in a charged micellar system - evidence of a shear-induced phase transition. Physical Review E, 56:1869-1879, 1997.

[26] R. W. Mair and P. T. Callaghan. Observation of shear banding in wormlike micelles by NMR velocity imaging. Europhysics Letters, 36:719-724, 1996.

[27] J. -B. Salmon, A. Colin, and S. Manneville. Velocity profiles in shear-banding wormlike micelles. Physical Review Letters, 90:228303(1)-228303(4), 2003.

[28] A. F. Mendez-Sanchez, M. R. Lopez-Gonzalez, V. H. Rolon-Garrido, J. PerezGonzalez, and L. de Vargas. Instabilities of micellar systems under homogeneous and non-homogeneous flow conditions. Rheologica Acta, 42:56-63, 2003. 
[29] Y. T. Hu and A. Lips. Kinetics and mechanism for shear banding in an entangled micellar solution. Journal of Rheology, 49(5):1001-1027, 2005.

[30] E. Miller and J. P. Rothstein. Transient evolution of shear-banding wormlike micelles solutions. Journal of Non-Newtonian Fluid Mechanics, 143:22-37, 2007.

[31] M. M. Britton and P. T. Callaghan. NMR visualisation of anomalous flow in coneand-plate rheometry. Journal of Rheology, 41:1365-1385, 1997.

[32] M. M. Britton and P. T. Callaghan. Two-phase shear band structures at uniform stress. Physical Review Letters, 78:4933-4937, 1997.

[33] M. M. Britton and P. T. Callaghan. Shear banding instability in wormlike micellar solutions. European Physical Journal B, 7:237-249, 1999.

[34] M. M. Britton, R. W. Mair, R. K. Lambert, and P. T. Callaghan. Transition to shear banding in pipe and couette flow of wormlike micelles solutions. Journal of

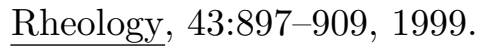

[35] E. Fischer and P. T. Callaghan. Is a birefringence band a shear band. Europhysics Letters, 50:803-809, 2000.

[36] M. R. Lopez-Gonzalez, W. M. Holmes, P. T. Callaghan, and P. J. Photinos. Shear banding fluctuations and nematic order in wormlike micelles. Physical Review Letters, 93:268302(1)-268302(4), 2004.

[37] J. -F. Berret, D. Roux, G. Porte, and P. Lindner. Shear induced isotropic-to-nematic phase transition in equilibrium polymers. Europhysics Letters, 25:521-526, 1994.

[38] J. -F. Berret, D. Roux, and G. Porte. Isotropic-to-nematic transition in wormlike micelles under shear. Journal de Physique II, 4:1261-1279, 1994.

[39] M. W. Liberatore, F. Nettesheim, N. J. Wagner, and L. Porcar. Spacially resolved small angle neutron scattering in the 1-2 plane: A study of shear induce phase separating wormlike micelles. Physical Review E, 73(2):504-507, 2006.

[40] J. P. Decruppe, E. Cappelaere, and R. Cressely. Optical and rheological properties of equimolar CTAB-KBr. Journal de Physique II, 7:257-270, 1997.

[41] J. -F. Berret, R. Gamez-Corrales, S. Lerouge, and J. P. Decruppe. Shear-thickening transition in surfactant solutions - new experimental features from rheology and flow birefringence. European Physical Journal E, 2:343-350, 2000.

[42] J. P. Decruppe, S. Lerouge, and J. -F. Berret. Insight in shear banding under transient flow. Physical Review E, 63:022501, 2001.

[43] J. P. Decruppe and A. Ponton. Flow birefringence, stress optical rule and rheology of four micellar solutions with the same low shear viscosity. European Physical Journal E, pages 201-207, 2003. 
[44] S. Lerouge, J. P. Decruppe, and P. Olmsted. Birefringence banding in a micellar solution or the complexity of heterogenous flows. Langmuir, 20:11355-11365, 2004.

[45] S. Lerouge, M. A. Fardin, M. Argentina, G. Gregoire, and O. Cardoso. Interface dynamics of shear banding flow of giant micelles. Softmatter, 4:1808-1819, 2009.

[46] V. Herle, J. Kohlbrecher, B. Pfister, P. Fischer, and E. J. Windhab. Alternating vorticity bands in a solution of wormlike micelles solutions. Physical Review Letters, 99(15):8302, 2007.

[47] P. Coussot, J. S. Raynaud, F. Bertrand, P. Moucheront, J. P. Guilbaud, H. T. Huynh, S. Jarny, and D. Lesueur. Coexistance of liquid and solid phases in flowing soft glassy materials. Physical Review Letters, 88(21):8301-8304, 2002.

[48] J. -B. Salmon, S. Manneville, and A. Colin. Shear banding a lyotropic lamellar phase. i. time averaged velocity profiles. Physical Review E, 68(5):1503-1514, 2003.

[49] S. Ravindranath and S.-Q. Wang. Steady state measurement in stress plateau region of entangled polymer solutions: Controlled-rate and controlled stress modes. Journal of Rheology, 52(4):957-980, 2008.

[50] P. E. Boukany and S.-Q. Wang. Exploring the transition from wall slip to bulk shear banding in well entangle DNA solutions. Softmatter, 5:780-789, 2009.

[51] P. E. Boukany, Y. T. Hu, and S.-Q. Wang. Observations of wall slip and shear banding in an entangle DNA solution. Macromolecules, 41(7):2644-2650, 2009.

[52] C. -Y. D. Lu, P. D. Olmsted, and R. C. Ball. Effects of nonlocal stress on the determination of shear banding flow. Physical Review Letters, 84(4):642-645, 2000.

[53] P. Olmsted, O. Radulescu, and C. -Y. D. Lu. Johnson-Segalman model with a diffusion term in cylindrical couette flow. Journal of Rheology, 44:257-275, 2000.

[54] E. Helfand and G. H. Fredrickson. Large fluctuations in polymer solutions under shear. Physical Review Letters, 62:2468-2471, 1989.

[55] C. -L. Wu, D. J. Pine, and P. K. Dixon. Enhanced concentration fluctuations in polymer solutions under shear flow. Physical Review Letters, 66(18), 1991.

[56] T. Hashimoto and T. Kume. "Butterfly" light scattering pattern in shear enhanced concetration fluctuations in polymer solutions and anomoly at high shear rates. Journal of the Physical Society of Japan, 61(6):1839-1843, 1992.

[57] P. Fischer, E. K. Wheeler, and G. G. Fuller. Shear-banding structure orientated in the voriticity direction observed for equimolar micellar solution. Rheologica Acta, 41:35-44, 2002.

[58] P. Fischer. Time dependent flow in equimolar micellar solutions: transient behaviour of the shear stress and firest normal stress difference in shear induced structures coupled with flow instabilities. Rheologica Acta, 39:234-240, 2000. 
[59] E. K. Wheeler, P. Fischer, and G. G. Fuller. A new class of shear-induced structure and flow instability in micellar solutions. Physical Review Letters, 1998.

[60] Y. T. Hu, P. Boltenhagen, E. Matthys, and D. J. Pine. Shear thickening in lowconcentration solutions of wormlike micelles. II. slip, fracture, and stability of the shear-induced phase. Journal of Rheology, 42(5):1209-1227, 1998.

[61] A. J. Liu and D. J. Pine. Shear induced gelation and fracture in micellar solutions. Physical Review Letters, 77(10):2121-2124, 1996.

[62] V. Herle, P. Fischer, and E. J. Windhab. Stress driven shear bands and the effect of confinement on their structures - a rheological, flow visualization, and rheo-SALS study. Langmuir, 21:9051-9057, 2005.

[63] S. Lerouge, J. P. Decruppe, and J. -F. Berret. Correlations between rheological and optical properties of a micellar solution under shear banding flow. Langmuir, 16:6464-6474, 2000.

[64] M. Ouchi, T. Takahashi, and M. Shirakashi. Shear-induced structure change and flow-instability in start-up couette flow of aqueous, wormlike micelle solution. Journal of Rheology, 50:341-352, 2006.

[65] H. Azzouzi, J. P. Decruppe, S. Lerouge, and O. Greffier. Temporal oscillations of the shear stress and scattered light in a shear-banding-shear-thickening micellar solution. European Physical Journal E, 17:507-514, 2005.

[66] B. A. Schubert, N. J. Wagner, E. W. Kaler, and S. R. Raghavan. Shear induced phase separation in solution of wormlike micelles. Langmuir, pages 3564-3573, 2004.

[67] P. D. Olmsted. Two-state shear diagrams for complex fluids in shear flow. Europhysics Letters, 48:339-345, 1999.

[68] J. L. Goveas and P. Olmsted. A minimal model for vorticity and gradient banding in complex fluids. European Physical Journal E, 6:78-89, 2001.

[69] S. M. Fielding and P. D. Olmsted. Flow phase diagrams for concentration coupled shear banding. European Physical Journal E, 11:65-83, 2003.

[70] S. M. Fielding and P. D. Olmsted. Early stages kinetics in a unified model of shear-induced demixing and mechanical shear banding instabilies. Physical Review Letters, 90(22):4501-4504, 2003.

[71] S. M. Fielding and P. D. Olmsted. Kinetics of the shear banding instability in startup flows. Physical Review E, 68(3):6313-6332, 2003.

[72] G. Porte, J. -F. Berret, and J. L. Harden. Inhomogenous flows of complex fluids mechanical instability vs non-equilibrium phase transition. Journal de Physique II, 7:459-472, 1997. 
[73] M. E. Cates and S. M. Fielding. Rheology of giant micelles. Advances in Physics, 55(7):799-879, 2006.

[74] R. Bandyopadhyay, G. Basappa, and A. K. Sood. Observation of chaotics dynmaics in dilute sheared aqueous solutions of CTAT. 84(9):2022-2025, 2000.

[75] R. Ganapathy and A. K. Sood. Intermittency route to rheochaos in wormlike micelles with flow-concentration coupling. Physical Review E, 96:108301, 2006.

[76] S. M. Fielding and P. D. Olmsted. Spacio-temporal oscillations and rheochaos in a simple model of shear banding. Physical Review Letters, 2003.

[77] S. M. Fielding. Linear instability of planar shear banding flow. Physical Review Letters, 95(13):4501-4504, 2005.

[78] S. M. Fielding and P. D. Olmsted. Non-linear dynamics of an interface between shear bands. Physical Review Letters, 96(10):4502-4505, 2006.

[79] S. M. Fielding. Complex dynamics of shear banded flow. Softmatter, 3:1262-1279, 2007.

[80] L. Becu, S. Manneville, and A. Colin. Spaciotemporal dynamics of wormlike micelles under shear. Physical Review Letters, 93:018301, 2004.

[81] D. J. Griffiths. Introduction to electrodynamics. Benjamin Cummings, United States of America, 2 edition, 1989.

[82] J. D. Jackson. Classical electrodynamics. Willey, United States of America, 3 edition, 1998.

[83] G. B. Arfken and H. J. Weber. Mathematical methods for physicists. Elsevier Academic Press, United Kingdom, 2005.

[84] M. V. Klein and T. E. Furtak. Optics. John Wiley and Sons, Ltd., 2 edition, 1986.

[85] R. M. A. Azzam and N. M. Bashara. Ellipsometry and polarised light. North Holland Publishing Company, Amsterdam, 2 edition, 1977.

[86] D. C. Champeney. Fourier transforms and their physical applications. Academic Press, United Kingdom, 1 edition, 1973.

[87] D. A. Weitz and D.J. Pine. Diffusing-wave spectroscopy. In Dynamic light scattering, pages 634-720. Clarendon Press, 1993.

[88] H. C. Van de Hulst. Light Scattering by Small Particles. John Wiley and Sons, Ltd., New York, 1957.

[89] D. Bicout and R. Maynard. Diffusing wave spectroscopy in inhomogeneous flows. Physica A, pages 387-411, 1993. 
[90] D. Bicout, E. Akkermans, and R. Maynard. Dynamical correlations for multiple light scattering in laminar flow. Journal de Physique I, 1:471-491, 1991.

[91] Wyn Brown. Light scattering - Principles and development. Oxford University Press, United States of America, 1996.

[92] C. H. Wang. Dynamic light scattering and viscoelasticity of a binary polymer solution. Macromolecules, 25:1524-1529, 1992.

[93] H. Rehage and H. Hoffmann. Rheological properties of viscoelastics surfactant systems. Journal of Physical Chemistry, 92:4712-4719, 1988.

[94] M. Buchanan, M. Atakhorrami, J. F. Palierne, and C. F. Schmidt. Comparing macrorheology and one- and two- point microrheology in wormlike micelles solutions. Macromolecules, 38:8840-8844, 2005.

[95] W. M. Holmes, M. R. Lopez-Gonzalez, and P. T. Callaghan. Fluctuations in shearbanded flow seen by NMR velocimetry. Europhysics Letters, pages 274-280, 2003.

[96] M. Atakhorrami and C. F. Schmidt. High-bandwidth one-and two-particle microrheology in solutions of wormlike micelles. Rheologica Acta, 45:449-456, 2006.

[97] M. Buchanan, M. Atakhorrami, J. F. Palierne, and C. F. Schmidt. High-frequency microrheology of wormlike micelles. Physical Review E, 72:011504, 2005.

[98] J. -F. Berret, G. Porte, and J. P. Decruppe. Inhomogeneous shears flows of wormlike micelles - a master dynamic phase diagram. Physical Review E, 55:1668-1677, 1997.

[99] M. M. Britton, R. W. Mair, R. K. Lambert, and P. T. Callaghan. Interface instabilities in shear-banding flow. Journal of Rheology, 43(4):897-909, 1999.

[100] J. -F. Berret, J. Appell, and G. Porte. Linear rheology of entangled wormlike micelles. Langmuir, 9:2851-2854, 1993.

[101] N. Z. Handzy and A. Belmonte. Oscillatory motion of rising bubbles in wormlike micellar fluids with different microstructure. 2003.

[102] M. R. Lopez-Gonzalez. Rheo-NMR of wormlike micelles. PhD thesis, Victoria, 2004.

[103] W. M. Holmes, M. R. Lopez-Gonzalez, and P. T. Callaghan. Shear induced constraint to amphiphile chain dynamics in wormlike micelles. Europhysics Letters, $66: 132-138,2004$.

[104] J. Y. Lee, G. G. Fuller, N. E. Hudson, and X. -F. Yuan. Investigation of shearbanding structure in wormlike micellar solution by point-wise flow-induced birefringence measurements. Journal of Rheology, 49:537-550, 2005.

[105] A. Shukla, R. Fuchs, and H. Rehage. Quasi-anomalous diffusion processes in entangled solutions of wormlike surfactant micelles. Langmuir, pages 3000-3006, 2006. 
[106] N. Willenbacher, C. Oelschlaeger, M. Schopferer, P. Fischer, F. Cardinaux, and F. Scheffold. Broad bandwidth optical and mechanical rheometry of wormlike micelle solutions. Physical Review Letters, 99(06):8302-8305, 2007.

[107] V. H. Rolon-Garrido, J. Perez-Gonzalez, and L. A. Vega Acosta Montalban. Vane rheometry of an aqueous solution of worm-like micelles. Revista Mexicana De Fisica, 49:29-39, 2003.

[108] F. Kern, P. Lemarechal, S. J. Candau, and M. E. Cates. Rheological properties of semidilute and concentrated aqueous solutions of CTAB-KBr. Langmuir, 8:437-440, 1992.

[109] E. K. Wheeler, P. Fischer, and G. G. Fuller. Time-periodic flow induced structures and instabilities in a viscoelastic surfactant solution. Journal of Non-Newtonian Fluid Mechanics, 75:193-208, 1998.

[110] B. R. Dasgupta, S.-Y. Tee, J. C. Crocker, B. J. Frisken, and D. A. Weitz. Microrheology of PEO using DWS and PCS. Physical Review E, 65:051505, 2002.

[111] D. Bicout and G. Maret. Multiple light scattering in Taylor-Couette flow. Physica A, 210:87-112, 1994.

[112] F. Cardinaux, L. Cipelletti, F. Scheffold, and P. Schurtenberger. Microrheology of giant-micelle solutions. Europhysics Letters, 57:738-744, 2002.

[113] B. S. Douglas, R. H. Colby, L. A. Madsen, and P. T. Callaghan. Rheo-NMR of wormlike micelles formed from nonionic pluronic surfactants. Macromolecules, 41(3):804$814,2008$.

[114] W. H. Press, S. A. Teukolsky, W. T. Vettering, and B. P. Flannery. Numerical Recipes in $\mathrm{C}++$ : The Art of Scientific Computing. Press Syndicate of the University of Cambridge, 2 edition, 2002.

[115] P. T. Callaghan. Principles of nuclear magnetic resonance microscopy. Clarendon Press, United Kingdom, 1 edition, 1991.

[116] X. -L. Xu, D. J. Pine, P. M. Chaikin, J. S. Huang, and D. A. Weitz. Diffusing-wave spectroscopy in a shear flow. Journal of the Optical Society of America B, 7:15-20, 1990 .

[117] R. H. Bartels, J. C. Beatty, and B. A. Barsky. An introduction to splines for use in computer graphics and geometric modelling. Morgan Kaufmann, United States of America, 1998.

[118] A. Raudsepp, P. T. Callaghan, and Y. Hemar. Shear localisation in stirred yoghurt. Rheologica Acta, 49:1435-1443, 2010. 
[119] S. Kumar and R. G. Larson. Shear banding and secondary flow in viscoelastic fluids between a cone and plate. Journal of Non-Newtonian Fluid Mechanics, 95(2):295$314,2000$.

[120] J. P. Decruppe, O. Greffier, S. Manneville, and S. Lerouge. Local velocity measurements in hetrogeneous and time-dependent flows of a micellar solution. Physical Review E, 73:061509(1)-061509(4), 2006.

[121] B. M. Marin-Santibanez, J. Perez-Gonzalez, L. de Vargas, F Rodriguez-Gonzalez, and G Huelsz. Rheometry-PIV of shear-thickening wormlike micelles. Langmuir, 22:4015-4026, 2006.

[122] J. P. Decruppe, R. Cressely, R. Makhoufi, and E. Cappelaere. Flow birefringence experiments showing a shear-banding structure in a CTAB solution. Colloids and Polymer Science, 273:346-351, 1995.

[123] E. K. Wheeler, P. Izu, and G. G. Fuller. Structure and rheology of wormlike micelles. Rheologica Acta, 35:139-149, 1996.

[124] C. Humbert and J. P. Decruppe. Flow birefringence and stress optical law of viscoelastic solutions of cationic surfactants and sodium salicylate. European Physical Journal E, 6:511-518, 1998.

[125] D. -G. Choi, W. -J. Kim, and S. -M. Yang. Shear induced microstructure and rheology of CPCl-NaSal micellar solutions. Korea-Australia Rheology Journal, 12:143$149,2000$.

[126] W. -J. Kim and S. -M. Yang. Microstructures and rheological responses of aqueous CTAB solutions in the presence of benzyl additives. Langmuir, 16:6084-6093, 2000.

[127] W. -J. Kim and S. -M. Yang. Effects of sodium salicylate on the microstructure of an aqueous micellar solution and its rheological responses. Journal of Colloid and Interface Science, 232:225-234, 2000.

[128] T. Takahashi, N. Yako, and M. Shirakashi. Relationship between shear-induced structure and optical anisotropy on CPyCl-NaSal aqueous solution. Journal of the Society of Rheology, Japan, 1:27-32, 2001.

[129] T. Takahashi, H. Sugata, and M. Shirakashi. Rheo-optic behavior of wormlike micelles under a shear-induced structure formational condition - verification of stressoptic rule by full component measurement of refractive index tensor. Journal of the Society of Rheology, Japan, 30:109-113, 2002.

[130] I. A. Kadoma and J. A. van Egmond. Shear-enhanced orientation and concentration fluctuations in wormlike micelles - effect of salt. Langmuir, 13:4551-4561, 1997.

[131] T. Hashimoto, T. Turukawa, and N. Mori. Flow property and micellar structures in capillary flows of surfactant solutions. Journal of the Society of Rheology, Japan, pages $1-8,2005$. 
[132] V. Schmitt, F. Lequeux, A. Pousse, and D Roax. Flow behaviour and shear induced transition near I-N transitions in equilibrium polymers. Langmuir, 10:955-961, 1994.

[133] D. C. Roux, J. -F. Berret, G. Porte, E. Peuvrel-Disdier, and P. Lindner. Shear induced orientations and textures of nematic wormlike micelles. Macromolecules, 28:1681-1687, 1995.

[134] J. -F. Berret, D. C. Roux, and P. Lindner. Structure and rheology of concentrated wormlike micelles at the shear induced isotropic-to-nematic transition. European Physical Journal B, 5:67-77, 1998.

[135] E. Fischer and P. T. Callaghan. Shear banding and the isotropic-to-nematic transition in wormlike micelles. Physical Review E, 64:011501, 2001.

[136] G. G. Fuller, J. M. Rallison, R. L. Schmidt, and L. G. Leal. The measurement of velocity gradients in laminar flow by homodyne light-scattering spectroscopy. Journal of Fluid Mechanics, 100(3):555-575, 1980.

[137] S. N. Schnatterly and S. E. Jasperson. An improved method for high reflectivity ellipsometry based on a new polarization method. Review of Scientific Instruments, 40:761-767, 1969.

[138] T. Shikata, S. J. Dahman, and D. S. Pearson. Rheo-optical behavior of wormlike micelles. Langmuir, pages 3470-3476, 1994.

[139] P. N. Pusey and W. van Megen. Dynamic light scattering by non-ergodic media. Physica A, 157, 1989.

[140] T. Takahashi, M. Shirakashi, K. Miyamoto, and G. G. Fuller. Development of a double-beam rheo-optical analyzer for full tensor measurement of optical anisotropy in complex fluid flow. Rheologica Acta, 41:448-455, 2002.

[141] T. Kume, T. Hashimoto, T. Takahashi, and G. G. Fuller. Rheo-optical studies of shear-induced structures in semidilute polystrene solutions. Macromolecules, 30:7232-7236, 1997.

[142] S. Saito, S. Koizumi, K. Matsuzaka, S. Suehiro, and T. Hashimoto. Light scattering and small angle neutron scattering studies of structures in a semidilute polymer solution induce under oscillatory shear flow. Macromolecules, 33:2153-2162, 2000.

[143] T. Yoshimura. Statistical properties of dynamic speckles. Journal of the Optical Society of America A, 3(7):1032-1054, 1986.

[144] T. Yoshimura, K. Nakagawa, and N. Wakabayashi. Rotational and boiling motion of speckles in a two-lens imaging system. Journal of the Optical Society of America A, 3(7):1018-1022, 1986.

[145] P. C. F. Moller, S. Rodts, M. A. J. Michels, and D. Bonn. Shear banding and yeild stress in soft glassy materials. Physical Review E, 77(4):1507, 2008. 
[146] T. Gibaud, C. Barentin, and S. Manneville. Influence of boundary conditions on yielding in soft glassy materials. Physical Review Letters, 101(2):8302-8305, 2008.

[147] T. Gibaud, C. Barentin, N. Taberlet, and S. Manneville. Shear induce fragmentation of laponite suspensions. Softmatter, 5(16):3026-3037, 2008. 


\section{Publications}

Three papers were published during the $\mathrm{PhD}$ :

1. A. Raudsepp, P. T. Callaghan and Y. Hemar. A study of nonlinear rheology of complex fluids using diffusing wave spectroscopy, Journal of Rheology, 52(5):1113-1129, 2008.

2. A. Raudsepp and P. T. Callaghan. A rheo-optical study of shear rate and optical anisotropy in wormlike micelles solutions, Softmatter, 4:784-796, 2008.

3. A. Raudsepp, K.W. Feindel and Y. Hemar. Shear localisation in stirred yoghurt. Rheologica Acta, 49(4):1435-1443, 2010.

The first paper relates to work presented in Chapter 7. The second paper relates to work discussed in Chapter 8. In the third paper, the flow-DWS technique developed in Chapter 7 was used to study the flow behaviour of stirred yoghurt. Work in this paper is largely ignored in the body of the thesis - the published paper is included here for completeness. 


\title{
Shear localisation in stirred yoghurt
}

\author{
Allan Raudsepp · Kirk W. Feindel · Yacine Hemar
}

Received: 10 December 2009 / Accepted: 16 January 2010

(C) Springer-Verlag 2010

\begin{abstract}
The shear flow behaviour of stirred yoghurt in the cone-and-plate and cylindrical Couette geometries was studied using diffusing wave spectroscopy (DWS) and nuclear magnetic resonance (NMR) velocimetry. Differences between the transmission and backscattering DWS correlations suggest the formation of a high shear rate band near the surface of a moving cone of a cone-and-plate geometry at low shear rates. At higher shear rates, homogeneous shear flow is indicated. NMR velocimetry unambiguously demonstrated that a high shear rate band forms at the moving inner wall of a cylindrical Couette geometry at low shear rates. At intermediate shear rate, a high shear rate band is formed at the stationary outer wall and pluglike flow is observed mid-gap. At higher shear rates, homogeneous shear flow is observed. Slip is seen at both walls. The three flow regimes appear to correlate loosely with transitions in the pseudo-steady-state flow curve and may reflect a break-up of the protein aggregates observed with confocal microscopy.
\end{abstract}

Keywords Shear localisation - Shear banding • Yoghurt $\cdot$ DWS $\cdot$ NMR velocimetry $\cdot$ Slip

A. Raudsepp $(\bowtie) \cdot$ K. W. Feindel

The MacDiarmid Institute for Advanced Materials and Nanotechnology, Victoria University of Wellington, Wellington, New Zealand

e-mail: allan.raudsepp@gmail.com

Y. Hemar

Food Science Australia, Werribee, Victoria, Australia

\section{Introduction}

Soft glassy materials are a broad class of complex fluids that include emulsions, foams, gels and colloidal glasses that possess a yield stress and exhibit both shear rejuvenation and aging (Moller et al. 2008). A general feature of the flow behaviour of these fluids is the formation of solid-like and fluid-like bands (Moller et al. 2008). Because shear flow is restricted to the fluid-like region, this partitioning results in 'shear localisation' (Gibaud et al. 2008). As is demonstrated here, stirred yoghurt shows shear induced fragmentation and a yield stress suggesting that shear localisation could be expected in this fluid.

Commercially, yoghurts are produced by the acidification of milk by bacterial cultures that ferment lactose to lactic acid (Lucey and Singh 1998). In milk, the primary proteins exist as smicelles comprised of four types of casein $\left(\beta-, \alpha_{\mathrm{s} 1^{-}}, \alpha_{\mathrm{s} 1}\right.$ and $\left.\mathrm{k}^{-}\right)$. It has been proposed that the proteins are held together by hydrophobic interactions and by calcium phosphate bridges (Horne 1998). At the surface of the casein micelle, a "hairy" layer made of $\kappa$-casein imparts a strong repulsive steric interaction that prevents casein micelle aggregation (de Kruif and Zhulina 1996). Lowering the $\mathrm{pH}$ to the isoelectric point of the casein, $\mathrm{pH}=4.6$, diminishes the net electrostatic charge and repulsive steric interactions, resulting in the aggregation of the casein micelles and the formation of a protein network.

Depending on the flow behaviour of the resulting protein network, yoghurt is described commercially as being firm, stirred or drinking (Yoon and McCarthy 2002). Rheologically, stirred yoghurt behaves like a thixotropic shear thinning fluid with an apparent yield stress (Basak and Ramaswamy 1994; Benezech 
and Maingonnat 1994). Rheological measurements of stirred yoghurt are known to be poorly reproducible due to sample preparation and sample-loading issues (Suwonsichon and Peleg 1999). Poor reproducibility has also been attributed to shear history and wall slip (Yoon and McCarthy 2002). Using magnetic resonance imaging, Yoon and McCarthy (2002) showed that both plug flow and a slip velocity are observed during the pipe flow of yoghurt. Slip velocity was found to increase with wall stress; that is, with the pressure difference across the pipe length. This has been recently confirmed by Henningsson et al. (2006) using crosscorrelation electrical resistance tomography.

In this paper, the flow behaviour of stirred yoghurt was studied using diffusing wave spectroscopy (DWS) and nuclear magnetic resonance (NMR) velocimetry in the cone-and-plate and cylindrical Couette geometries. NMR has been used to examine the flow behaviour of many complex fluids including foodstuffs (Britton and Callaghan 1997) and shear banding fluids (Callaghan 2008). DWS is a dynamic light scattering technique in which the propagation of light through a highly multiply scattering medium is treated as a diffusive process (Weitz and Pine 1992). The technique is commonly used to characterise the linear rheology of complex fluids through measurement of the diffusion of embedded probe particles. Here, we utilise the technique's sensitivity to shear rate and exploit the intrinsic turbidity of yoghurt to explore the fluid's flow behaviour in a conventional geometry commonly used in rotational rheological measurements.

\section{Experimental}

Stirred yoghurt (Biofarm Products Ltd, Palmerston North, New Zealand) was studied using confocal microscopy, rheometry, flow-DWS and NMRvelocimetry. The yoghurt was made from whole milk and used as received.

Confocal scanning laser microscopy (CSLM) was performed on a Leica microscope (TCS SP5 DM6000B) in fluorescence mode with a DPSS 561 laser (excitation wavelength of $\lambda=561 \mathrm{~nm}$, emission spectrum $\lambda=565$ $659 \mathrm{~nm})$ and an oil-immersion objective $(\times 100)$. Images were recorded with $1024 \times 1024$ pixel resolution. The protein network was dyed with Fast-green by the addition of $6 \mu \mathrm{l}$ of dye, from a $0.2 \% w / w$ mother solution, to $1 \mathrm{ml}$ of yoghurt sample. Yoghurt samples were observed with CSLM before and after extensively stirring the sample with a magnetic stirrer.
Rheometry was performed on a controlled stress TA AR2000 operated in strain controlled mode. Several measurement geometries were used.

The experimental set-up for the flow DWS experiment is shown in Fig. 1. Light from a polarised laser (L, $15 \mathrm{~mW} \lambda=632 \mathrm{~nm}$, Melles-Griot) was directed through a beam expander, a variable neutral density filter (Edmund optics) and reflected by a gold mirror into a rheometer (TA AR2000) mounted homemade optical cone and plate geometry. Both the Perspex cone (60 $\mathrm{mm}$ diameter, $4^{\circ}$ cone angle) and glass plate were transparent. Scattered light was collected through a polariser crossed with the laser, mounted on a GRIN lens, directed by a fibre optic into a fibre optic beam splitter (Font) into two photon counting photomultipliers (www.correlator.com). The signal from the two photomultipliers was cross-correlated at a hardware correlator (www.correlator.com) and acquired for analysis at the host PC. By repositioning the mirror, light could be directed through the cone to the plate (CP) or through the plate to the cone (PC). By repositioning both the mirror and collection optics, four DWS measurement configurations were possible: (1) mirror and collection optics above (CP backscattering geometry), (2) mirror above and collection optics below (CP transmission geometry), (3) mirror and collection optics below (PC backscattering geometry) and (4) mirror below and collection optics above (PC transmission geometry). In all measurements, light was directed into the sample and scattered light collected from the sample $18 \mathrm{~mm}$ from the axis of rotation of the cone.

NMR velocimetry (Callaghan 1991) measurements were performed using a $9.4 \mathrm{~T}$ vertical wide-bore superconducting magnet equipped with a Bruker Avance 400 NMR spectrometer and a Bruker Micro-2.5 triaxial gradient unit. Coupled with GREAT-60 current
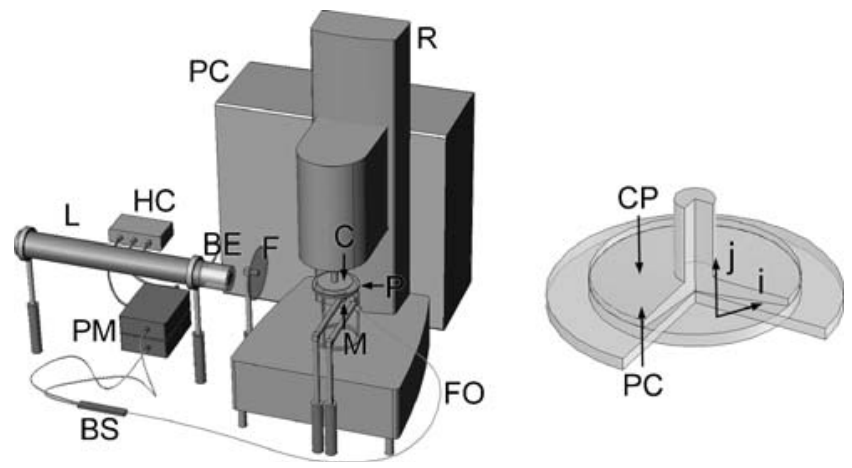

Fig. 1 Flow DWS setup and schematic of the optical geometry used for the DWS experiments. Arrows labelled CP, and PC indicate the direction of incidence 
amplifiers, the maximum pulsed magnetic field gradients achievable are $1.5 \mathrm{~T} \mathrm{~m}^{-1}$. All experiments were performed with a ${ }^{1} \mathrm{H}$-tuned (ca. 399.7 MHz) Bruker SAW coil. NMR velocity measurements were obtained with a volume-selective one-dimensional imaging sequence preceded by a pulsed-gradient stimulated echo (Fig. 2). The ${ }^{1} \mathrm{H} T_{1}$ and $T_{2}$ relaxation times for the yoghurt were measured to be $\sim 2.3 \mathrm{~s}$ and $\sim 90 \mathrm{~ms}$, respectively. Therefore, an excitation pulse of $\sim 80^{\circ}$ $(50 \mu \mathrm{s})$ was employed with a delay of $3.5 \mathrm{~s}$ between experiments. Velocity- or $q$-space was probed by repeating the experiment for eight values of the $q$-gradient, ranging from 0 to $0.3625 \mathrm{~T} \mathrm{~m}^{-1}$. The duration of the q-gradient, $\delta$, ranged from 1 to $2 \mathrm{~ms}$, and the observation period between $q$-gradients, $\Delta$, ranged from 10 to $75 \mathrm{~ms}$. Volume selection was performed with two bandselective 1-ms-shaped RF pulses in the presence of a magnetic field gradient which defined the physical slice thickness. During acquisition of the signal, a magnetic field gradient was applied across the diameter of the Couette cell to spatially resolve the flow profile across the gap (25.3 mm field of view). To limit water-fat chemical shift artefacts, a large acquisition bandwidth of $500 \mathrm{kHz}$ was employed to acquire 512 complex points. The nominal voxel size was $2 \mathrm{~mm} \times 50 \mu \mathrm{m} \times$ $20 \mathrm{~mm}$ (flow direction $\times$ across gap $\times$ depth). A fourstep CYCLOPS phase cycle (Hoult and Richards 1975) was employed for each $q$-step, resulting in a total time of ca. 2 min per velocity image. The $q$-dimension was zero-filled to 1024 and Fourier transformed to yield a propagator for each voxel of the one-dimensional NMR image. The location of the propagator peak maximum was taken to represent the average voxel velocity sampled over the duration of the NMR measurement.

Strain-rate-controlled shear deformation was applied with a Magritek Rheo-NMR accessory with the drive shaft coupled to the inner cylinder of a custom made cylindrical Couette cell. The Couette cell was constructed from glass NMR tubes (Wilmad Glass) with outer and inner diameters of 25/23 and 20/18 mm (concentric to $50 \mu \mathrm{m}$ ), resulting in a nominal gap width of $1.5 \mathrm{~mm}$. A test sample, placed in the gap between the tubes, is sheared by the rotation of the inner NMR tube. The apparent shear rate in this geometry is equal to the linear velocity of the inner wall divided by the gap width. An unsheared marker fluid is placed in the centre of the inner tube to elucidate slip.

\section{Measurement and analysis}

\section{Confocal microscopy}

Confocal micrographs of the yoghurt are shown in Fig. 3. The milk proteins are stained by the Fast-green dye and appear white in the images. These micrographs clearly show that the yoghurt is made of a network of aggregated or flocculated milk proteins containing large voids (dark region) filled with milk serum. After extensive stirring the size of the protein aggregates is markedly reduced.

\section{Rheometry}

The pseudo-steady-state flow curve of the yoghurt was measured by stepping the apparent shear rate from either $\dot{\gamma}=0.01$ or $0.0316 \mathrm{~s}^{-1}$ in 10 steps per decade increments with a 2-min dwell time at each shear rate. Measurements were made using a (1) $40 \mathrm{~mm} 2^{\circ}$ factory acrylic cone and steel plate, (2) $60 \mathrm{~mm} 4^{\circ}$ factory steel cone and steel plate or (3) $60 \mathrm{~mm} 4^{\circ}$ homemade Perspex cone and glass plate. The shear stress was measured continuously. Measurements are shown in Fig. 4i. The differences observed between measurements made with the $2^{\circ}$ and $4^{\circ}$ cones suggest a dependence on cone

Fig. 2 NMR velocity imaging pulse sequence and schematic of the cylindrical Couette geometry used for the NMR experiments
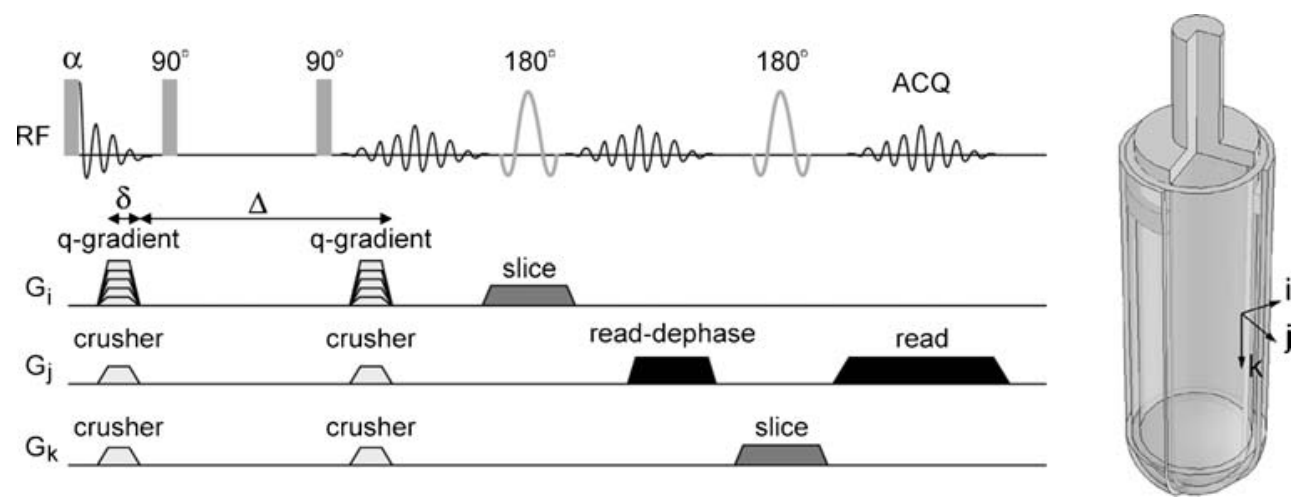
Fig. 3 Confocal micrographs of the yoghurt samples $i$ before and $i i$ after stirring

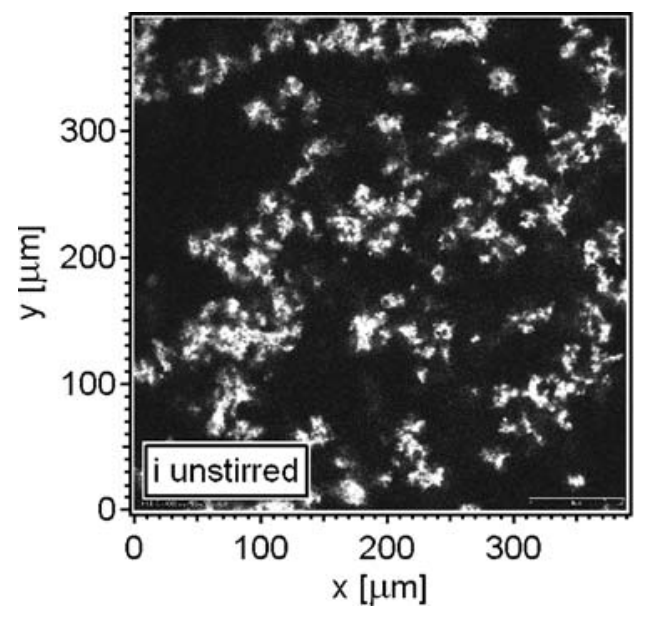

angle. The measured pseudo-steady-state flow curves can loosely be divided into three regions. At the lowest shear rates, $\dot{\gamma}<0.4-1.2 \mathrm{~s}^{-1}$ (depending on geometry), plateau-like or a decreasing stress is observed with increasing shear rate in the flow curve. A stress plateau has been observed in soft glassy materials and has been correlated to partial fluidisation and shear banding (Rogers et al. 2008; Moller et al. 2008). The plateau-like region observed here might be associated with the localised fragmentation of the protein network seen in the confocal microscopy. At intermediate shear rates, stress scales $\sigma \propto \dot{\gamma}^{0.45}$ while at highest shear rates, $\dot{\gamma}>8-13 \mathrm{~s}^{-1}$, stress scales according to $\sigma \propto \dot{\gamma}^{0.30}$. The data are replotted with time in Fig. 4ii and iii and show that the sample exhibits both thixotropy and rheopexy. Additional measurements made with the $40 \mathrm{~mm} 2^{\circ}$ factory acrylic cone, in which stress was stepped in 10 steps per decade increments from $0.01 \mathrm{~Pa}$, indicated that no measurable flow was present until the stress exceeded a yield stress of $1 \mathrm{~Pa}$. This correlates well with the plateau stress at low shear rate in controlled strain rate measurements.

\section{Flow DWS}

In DWS, a normalised intensity correlation

$\langle I(0) I(t)\rangle /\langle I\rangle^{2}=1+\beta\left|g_{1}(t)\right|^{2}$

is measured. $\beta$ is a coherence factor that can be determined from the short time behaviour of the normalised intensity correlation, and $g_{1}(t)$ is the field correlation function. The time dependence of the intensity correlation function depends on the relative motion of the embedded scattering centres. For simple shear flow, the field correlation function is given by (Bicout and Maret 1994).

$$
g_{1}(t)=\sum_{n=1}^{\infty} G_{n}\left(y_{i}, y_{\mathrm{o}}\right) \exp \left[-2 n k^{2} D t-\frac{1}{15} n\left(k l^{*} \dot{\gamma} t\right)^{2}\right]
$$
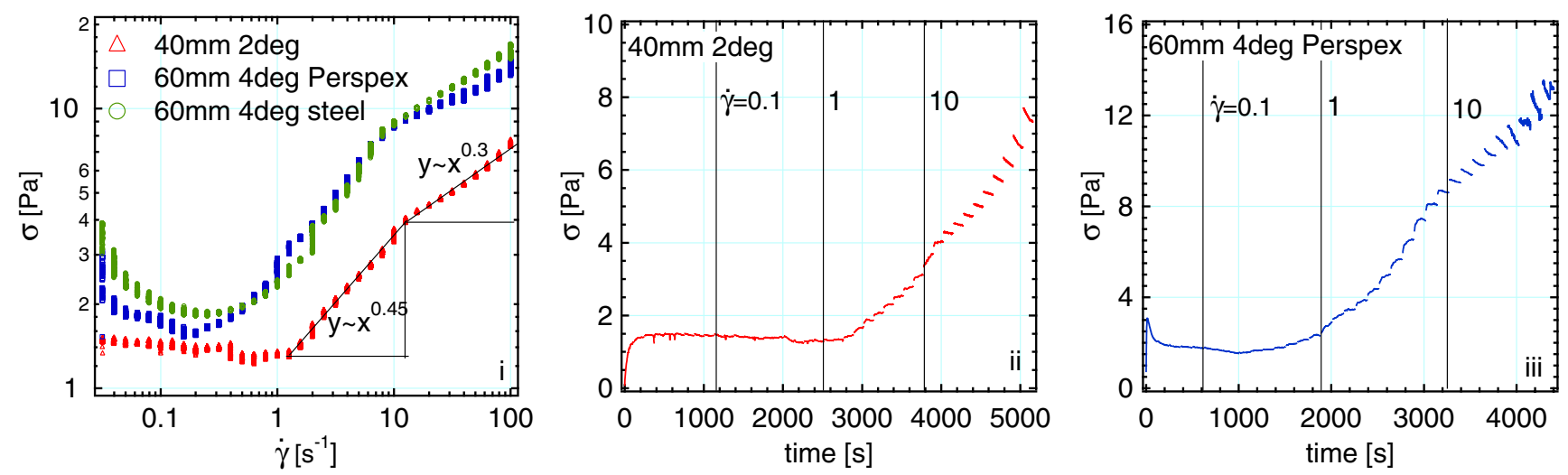

Fig. 4 Pseudo-steady-state flow curves showing dependence on $i$ shear rate and $i i$, iii time 
Here, $k$ is the wavenumber in the medium, $D$ is the diffusion constant of the embedded scattering centres, $\dot{\gamma}=d v_{x} / d y=$ constant is the shear rate and $l^{*}$ is characteristic length scale that describes the transport mean free path of light in the medium. $l^{*}$ is a measure of the system's turbidity and depends on the scattering centres' size, refractive index and volume fraction (Weitz and Pine 1992). $G_{n}\left(y_{i}, y_{o}\right)$ is a propagator describing the probable distribution of steps $n$ of step size $l^{*}$ required to randomly walk between the points $y_{i}$ and $y_{o}$. This random walk represents the path of light diffusing through the medium. $y_{i}$ is the point in the medium where light starts to diffuse and $y_{o}$ the point at which light exits the medium and is subsequently detected. For uniform illumination of an infinite slab of thickness $L, G_{n}\left(y_{i}, y_{o}\right)$ has the form:

$$
\begin{aligned}
G_{n}\left(y_{i}, y_{o}\right)= & \frac{2}{L} \sum_{m=1}^{\infty} \sin \left[\frac{m \pi y_{i}}{L}\right] \sin \left[\frac{m \pi y_{\mathrm{o}}}{L}\right] \\
& \times \exp \left[-m^{2} \frac{n}{n_{0}}\right]
\end{aligned}
$$

where $n_{0}=3 L^{2} / \pi l^{* 2}$ (Bicout and Maynard 1993). Two experimental geometries are commonly used in DWS: (1) backscattering in which light is detected from the incident side of the slab and (2) transmission where light is detected from the opposite side. In the backscattering geometry, it is commonly assumed that $y_{i}=l^{*}$ and $y_{o}=\gamma_{0} l^{*}$ whereas in the transmission geometry, it is assumed that $y_{o}=L-\gamma_{0} l^{*} \cdot \gamma_{0}$ describes the diffusion of light near an interface. It was measured experimentally as $\gamma_{0}=1.7$ for a glass/water interface in Bicout and Maret (1994); here, we assume a value $\gamma_{0}=1.7$ here. Depth sensitivity to diffusion or shear induced relative motion of the embedded scattering centres is set by the propagator. In the backscattering geometry, short paths are favoured. As a consequence, backscattering DWS measurements preferentially probe relative motion near the surface. In the transmission geometry, longer paths are favoured as light paths must traverse the entire slab to be detected, and the measurement is closer to a spatial average.

Measurements in two backscattering and two transmission geometries are shown in Fig. 5. The apparent shear rate was stepped from $\dot{\gamma}=0.0316$ to $100 \mathrm{~s}^{-1}$ in 10 per decade increments with a dwell time of 2 min at each shear rate step. The red arrow indicates the direction of incidence and $d$ the position of the collection optics. The correlations reported are an average over the last $30 \mathrm{~s}$ at each shear rate step. The stress was measured concurrently; an example of the stress measured with the Perspex cone is reported in Fig. 5i and iii.

To analyse the data, it was assumed that uniform shear flow was achieved for apparent shear rates $\dot{\gamma}>$ $10 \mathrm{~s}^{-1}$. With the contribution of diffusion neglected, the transmission and backscattering correlations measured above $\dot{\gamma}>10 \mathrm{~s}^{-1}$ were simultaneously fitted to Eqs. 2
Fig. 5 Measured flow DWS correlations in the four optical configurations
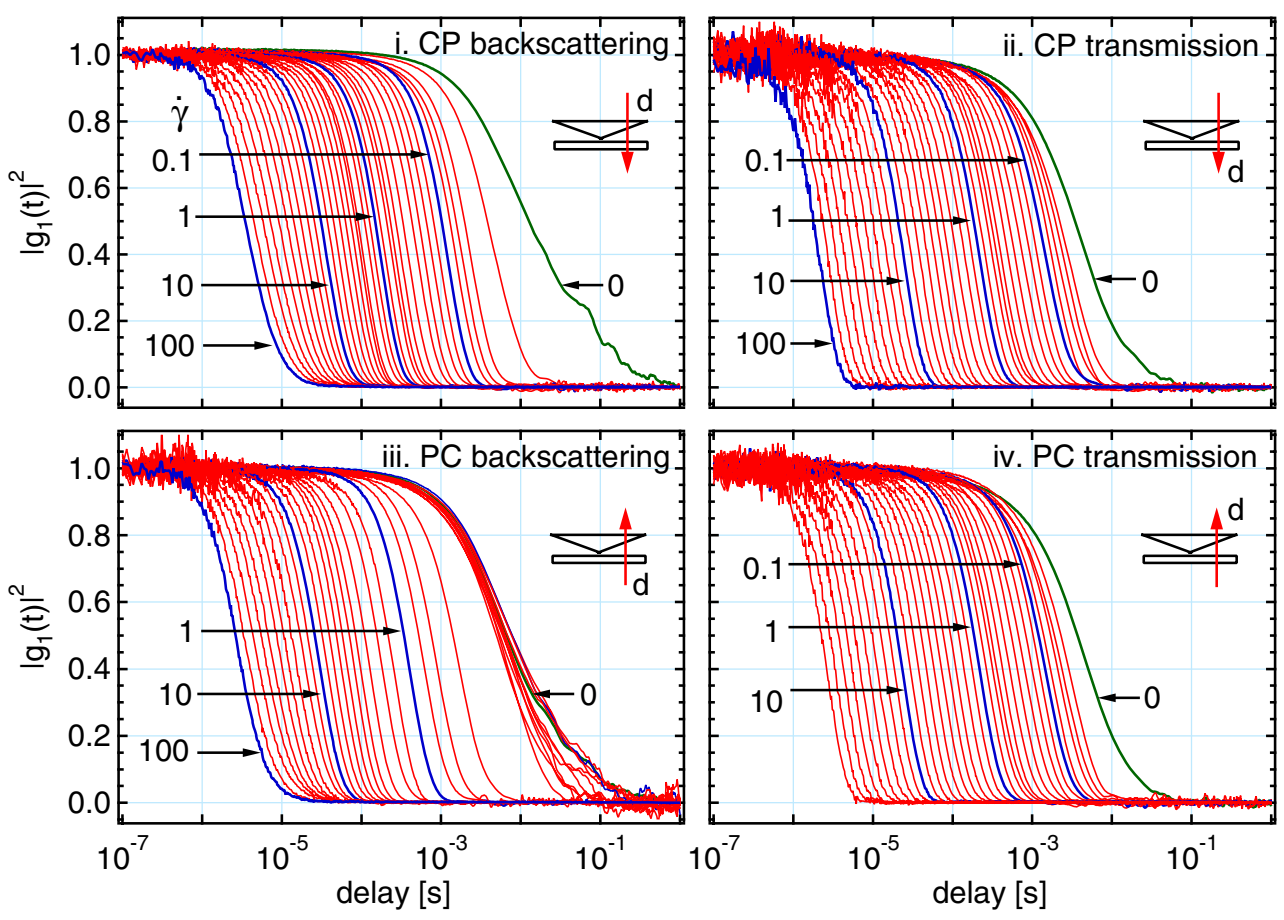


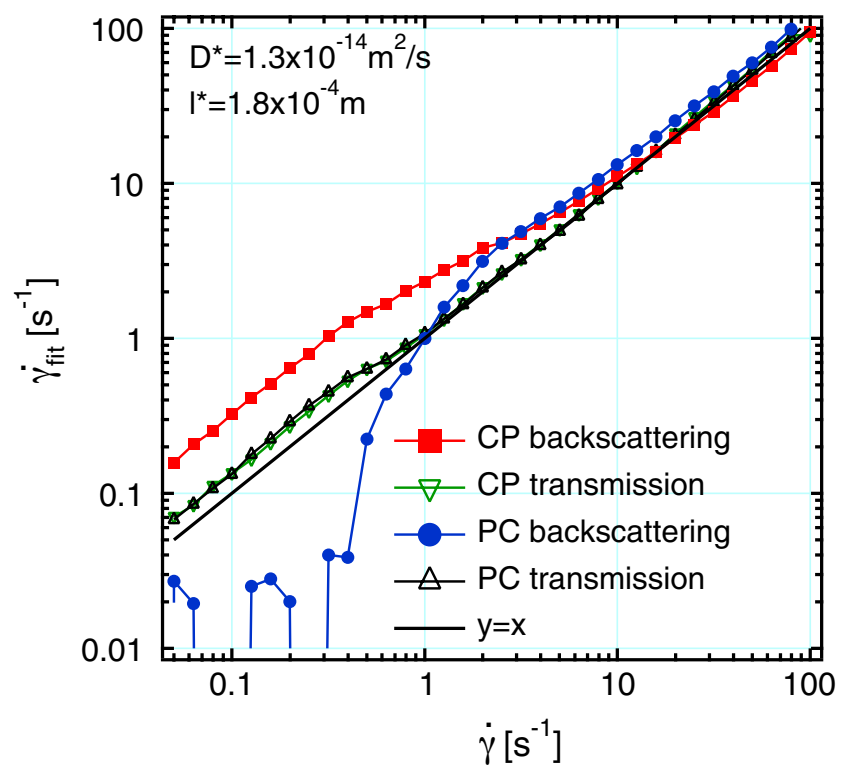

Fig. 6 Shear rate estimated by fitting the DWS correlations measured in the four experimental DWS geometries

and 3 to determine a best fitting $l^{*}=0.18 \mathrm{~mm}$ for the yoghurt. Using this $l^{*}$ value, the correlations measured in the absence of shear $\left(\dot{\gamma}=0 \mathrm{~s}^{-1}\right)$ were fitted to determine an average effective diffusion constant $D^{*}=1.3 \times 10^{-14} \mathrm{~m}^{2} / \mathrm{s}$ for the scattering centres in the yoghurt. Measurements at intermediate shear rates were then fitted to Eqs. 2 and 3 to determine $\dot{\gamma}=\dot{\gamma}_{\text {fit }}$. Because $l^{*}$ may depend on shear rate and may vary spatially through the yoghurt sample and because $\gamma_{0}$ and absorbance were not determined experimentally, this method of analysis is essentially semi-quantitative (for a more quantitative approach, see Raudsepp et al. 2008).

$\gamma_{\text {fit }}$ is shown in Fig. 6. At low apparent shear rates, $\dot{\gamma}<0.5 \mathrm{~s}^{-1}$, the fitted shear rate $\dot{\gamma}_{\text {fit }}$ reported at the stationary plate in the $\mathrm{PC}$ backscattering configuration (Fig. 5iii) is significantly lower than that reported at the moving cone in $\mathrm{CP}$ backscattering configuration (Fig. 5i). An intermediate value is reported by $\mathrm{PC}$ and $\mathrm{CP}$ transmission configurations (Fig. 5ii and iv). As noted above, the backscattering measurements preferentially probe the local shear rate near the surface, whereas the transmission measurements report on a spatial average across the gap-these observations are consistent with the formation of a solid-like low shear band at the stationary plate and a fluid-like high shear band at the moving cone. Around $\dot{\gamma}=0.5 \mathrm{~s}^{-1}, \dot{\gamma}_{\text {fit }}$ near the plate
Fig. 7 Velocity profiles measured using NMR-velocimetry at $i$ low (solid lines), $i i$ intermediate (solid lines) and iii high shear rates (solid lines). Dashed lines correspond to other profiles measured during the run. iv 3D visualisation of data shown in (ii) and (iii)
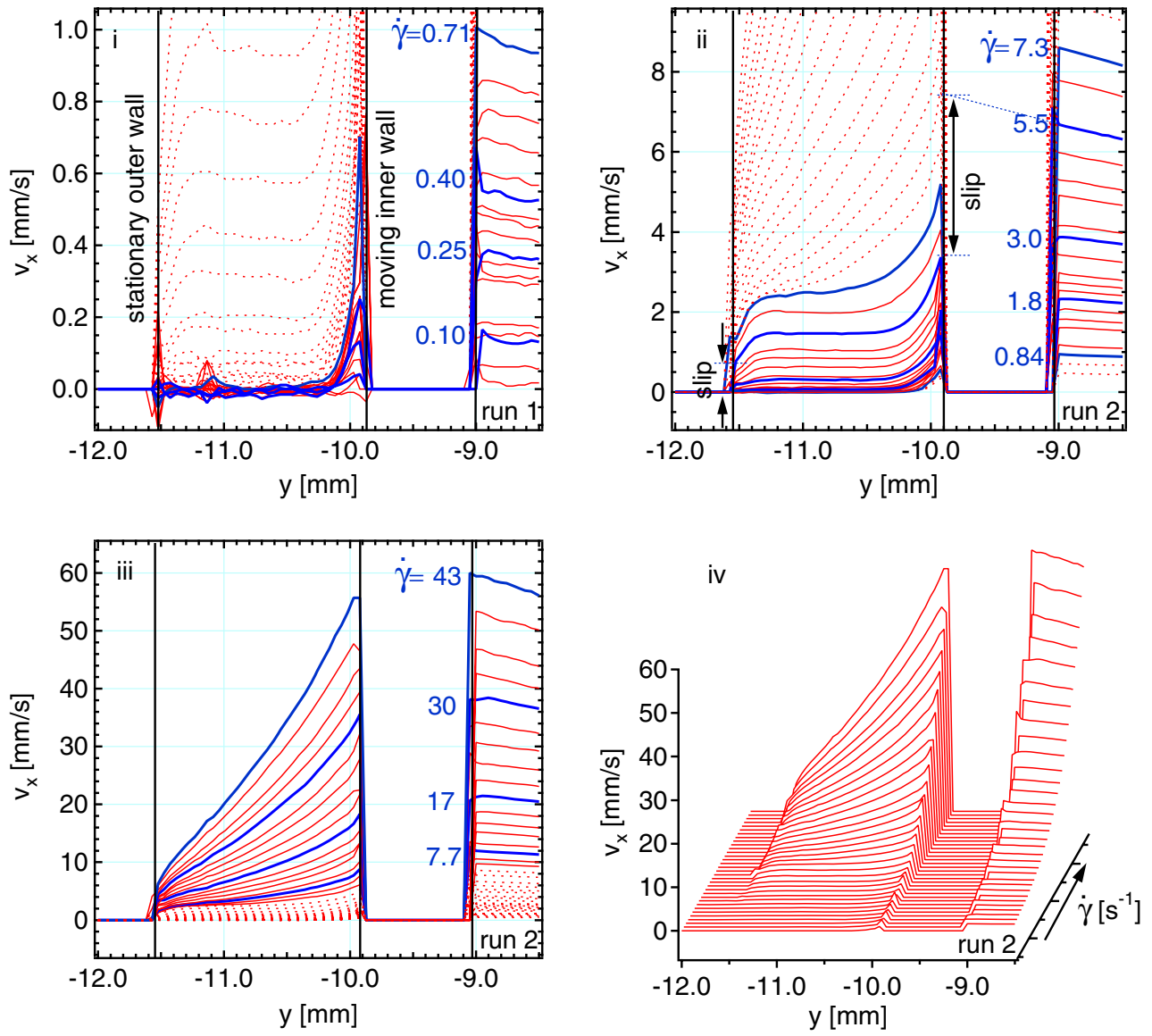
begins to grow sharply with increasing $\dot{\gamma}$. At intermediate shear rates, $\dot{\gamma}=0.5-5 \mathrm{~s}^{-1}, \dot{\gamma}_{\text {fit }}$ near the plate continues to grow while a small reduction in $\dot{\gamma}_{\text {fit }}$ near the cone is observed. At high shear rates, $\gamma>5 \mathrm{~s}^{-1}, \dot{\gamma}_{\text {fit }}$ reported by both the backscattering and transmission measurements is very similar suggesting that homogeneous shear flow is present.

\section{NMR velocimetry}

Velocity profiles of the yoghurt sheared in the cylindrical Couette geometry are reported in Fig. 7. In these measurements, the apparent shear rate was stepped from a $\dot{\gamma}=0.04$ to 5.61 (run 1 ) or $\dot{\gamma}=0.33-43 \mathrm{~s}^{-1}$ (run 2) in approximately 20 per decade increments with a dwell time of 6 min at each shear rate. The profiles reported are an average over this 6-min period. At low shear rates, $\dot{\gamma}<0.8 \mathrm{~s}^{-1}$ (Fig. 7i, solid lines), a localised high shear rate band forms at the moving inner wall. At intermediate shear rates, $\dot{\gamma}=0.8-7 \mathrm{~s}^{-1}$ (Fig. 7ii, solid lines), a localised high shear band emerges at the stationary outer wall of the geometry. The local shear rate is close to zero mid-gap over this shear rate range.

At higher shear rates, $\dot{\gamma}>8 \mathrm{~s}^{-1}$ (Fig. 7iii, solid lines), the mid-gap local shear rate increases as the shear flow becomes more homogeneous.

In the absence of slip, the expected velocity at the outer wall is zero. The expected velocity at the inner wall $v_{\mathrm{m}}$ can be inferred by linearly extrapolating the velocity of the marker fluid to the inner wall of the gap. The measured velocity at the outer wall $v_{\mathrm{o}}$, inner wall $v_{\mathrm{i}}$ and extrapolated velocity $v_{\mathrm{m}}$ are shown in Fig. $8 \mathrm{i}$ and the ratio $v_{\mathrm{o}} / v_{\mathrm{m}}$ and $v_{\mathrm{i}} / v_{\mathrm{m}}$ in Fig. 8ii. At lower shear rates, significant slip is observed at the moving inner wall. At higher shear rates, $v_{\mathrm{i}} / v_{\mathrm{m}}$ increases towards

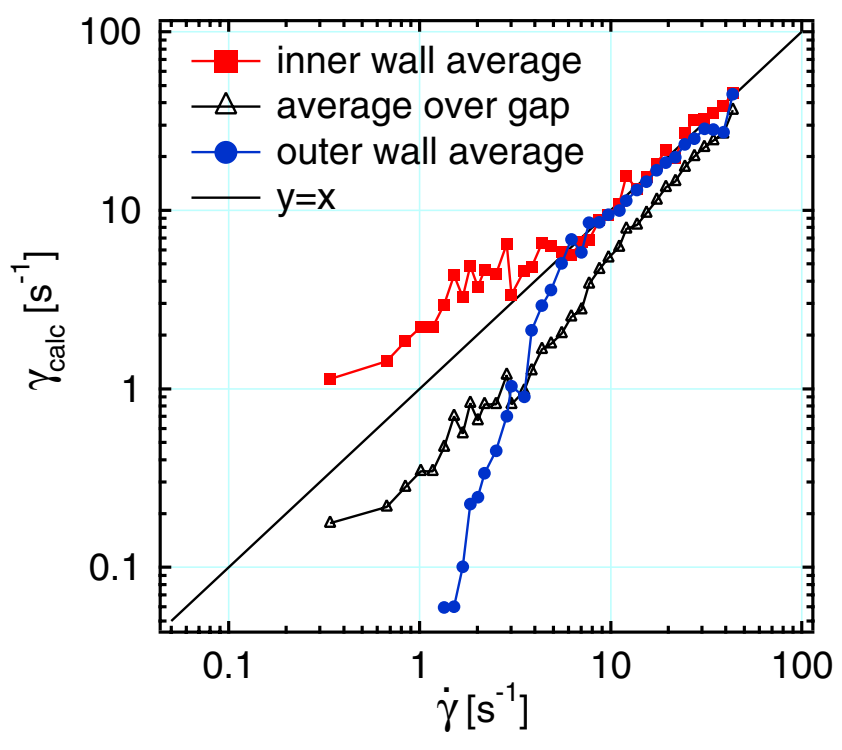

Fig. 9 Average shear rates calculated from the NMR velocity profile near the inner and outer walls of the geometry and over the entire gap

$v_{\mathrm{i}} / v_{\mathrm{m}}=1$ expected in the absence of slip. While slip appears to be absent at the outer wall at lower shear rates, it is observed at intermediate and higher shear rate saturating at a value $v_{\mathrm{o}} / v_{\mathrm{m}} \approx 0.15$.

In order to compare the NMR with the DWS measurements, the velocity profile is differentiated for apparent shear rates in the range $\dot{\gamma}=0.33-43 \mathrm{~s}^{-1}$ and an average shear rate, $\dot{\gamma}_{\text {calc }}$, is calculated over a $0.25-\mathrm{mm}$ region nearest the inner wall, a $0.25-\mathrm{mm}$ region nearest the outer wall and an average over the entire gap. These averages are shown in Fig. 9. The inner wall and outer wall averages show similar behaviour to the fitted shear rate for the backscattering measurements, albeit shifted by a factor of approximately 2 to 3 to higher apparent shear rates.
Fig. 8 Slip observed at the inner and outer walls of the gap
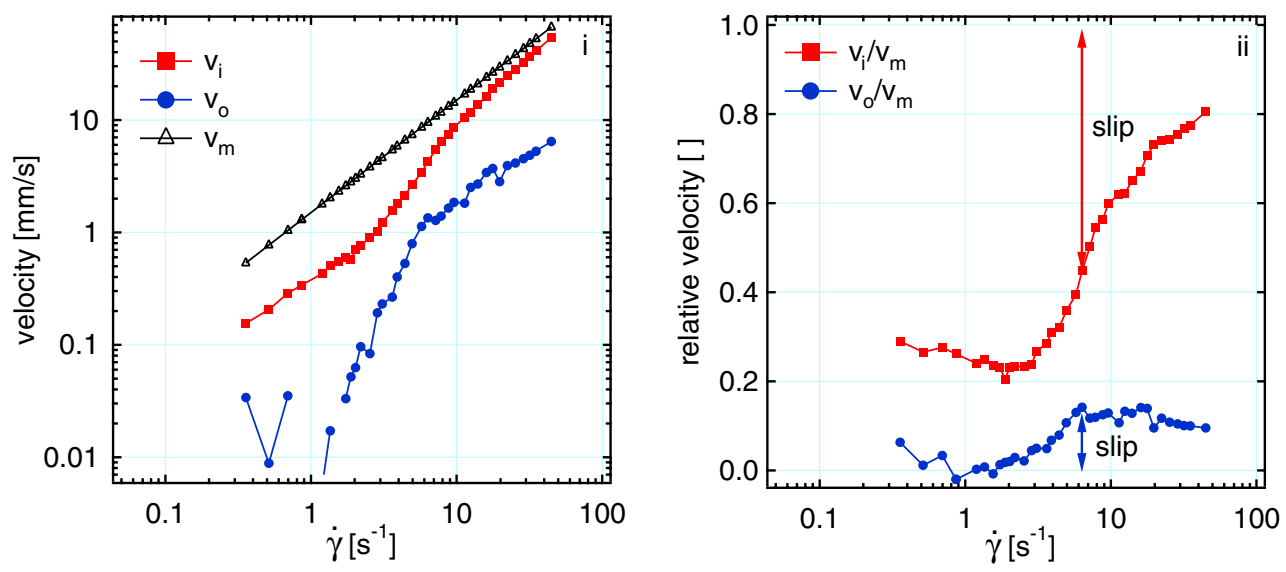


\section{Discussion}

The flow DWS and NMR velocimetry measurements are easily reconciled at low and high apparent shear rates. At low shear rates, an unsheared solid-like band is observed at the outer wall, and a high shear rate fluidlike band is seen at the moving wall in the NMR measurements (Fig. 6i). These observations are consistent with the backscattering flow DWS measurements (Fig. 5i and iii) that indicate local shears rates of $\gamma_{\text {fit }} \approx 0$ near the stationary plate and $\dot{\gamma}_{\text {fit }}>\dot{\gamma}$ near the moving cone (Fig. 6). At high apparent shear rates, the NMR velocimetry indicate that increasingly homogeneous shear flow is present (Fig. 7iii). Increasingly homogeneous shear flow is also indicated by flow DWS as $\gamma_{\text {fit }}$ converges to the apparent shear rate in both the backscattering and transmission geometries (Figs. 5 and 6). At intermediate shear rates, the NMR velocimetry measurements clearly show that high shear rate bands are present at both the moving and the stationary walls of the cylindrical Couette geometry and are connected by a mid-gap band having shear rate close to zero (Fig. 8ii). Although the flow DWS indicated that there is indeed shear flow near both the moving and the stationary walls of the cone-and-plate geometry in this regime (see Fig. $6,0.5 \mathrm{~s}^{-1} \leq \dot{\gamma} \leq 3 \mathrm{~s}^{-1}$ ), the technique, which reports a weighted spatial average, is unable to resolve such complex flow behaviour. The correspondence between the flow DWS and NMR velocimetry measurements (Figs. 6 and 9) indicates that flow DWS can provide at least semi-quantitative information about the flow behaviour of an unknown complex fluid. In this instance, the flow DWS measurements motivated a more quantitative study using NMR velocimetry.

Both the flow DWS and NMR velocimetry measurements indicated that the yoghurt exhibits shear localisation in both the cone-and-plate and cylindrical Couette geometries. Three regimes were identified in the flow curve of the yoghurt-these regimes appear to correlate loosely to changes in the flow behaviour.

Wall slip and plug flow have been observed in yoghurt in the pipe flow geometry by others (Yoon and McCarthy 2002; Henningsson et al. 2006). Wall slip and plug-like flow were observed in the cylindrical Couette geometry here. In the pipe flow geometry, the solid-like plug, corresponding to a region flowing with a constant velocity, formed in the low stress region in the middle of the pipe. Surprisingly, in the cylindrical Couette geometry used here, the solid-like 'plug' formed in a region of intermediate stress in the middle of the gap.

In a shear banding fluid, viscosity spatially bifurcates in the gradient flow direction. This partitioning into regions of high and low viscosity results in bands of low and high shear rate (Olmsted 2008). Shear banding has been studied extensively in wormlike micelles solutions. In the cylindrical Couette geometry, the fluid is commonly observed to partition into a high and low shear rate band in the vicinity of a stress plateau in controlled strain rate conditions. Here, the high shear rate band (of strain rate $\dot{\gamma}_{\mathrm{HI}}$ ) is observed to form at the higher stress inner wall and expands into the low shear rate band (of strain rate $\gamma_{\mathrm{LO}}$ ) with increasing gapaverage strain rate $\gamma$ with a fractional width $x$ given by lever rule $\gamma=x \gamma_{\mathrm{HI}}+(1-x) \gamma_{\mathrm{LO}}$ (Salmon et al. 2003). Experimentally, there is evidence that soft glassy materials partition into fluid-like and solid-like shear rate bands (of strain rates $\dot{\gamma}_{\text {fluid }}$ and $\dot{\gamma}_{\text {solid }}=0 \mathrm{~s}^{-1}$, respectively) according to the lever rule $\gamma=x \gamma_{\text {fluid }}$ in both the cylindrical Couette (Gibaud et al. 2009) and cone-andplate (Moller et al. 2008) geometries with the fluid-like bands forming in the high stress region of the geometry in the vicinity of the stress plateau in controlled strain rate conditions. Although a stress plateau was observed at low shear rates for the yoghurt, partitioning into solid-like and fluid-like bands according to the lever rule was not observed here.

Recently, Gibaud et al. (2008) examined the role of wall slip on flow behaviour of soft glassy materials. Here, ultrasonic velocimetry was used to profile flow in a soft glassy Laponite suspension in both a rough wall and smooth wall cylindrical Couette geometry in constant strain rate conditions. In the rough wall geometry, slip was absent and material partitioned into solid-like and fluid-like regions as expected. Flow in the smooth wall geometry was significantly more complicated. The solid-like phase was observed to detach from the outer wall of the geometry and flow as a discontinuous plug in the centre of the gap. Over time, the plug was observed to gradually erode. Significant wall slip-stick was observed at both the inner and outer walls of this smooth walled geometry. Both wall slip and plug flow were observed in the NMR velocimetry measurements of the yoghurt at intermediate shear rates here suggesting that the solid-like region, observed at the outer wall of the cylindrical Couette at low shear rates, is detaching from this wall and flowing mid-gap as a plug. Viscous flow may be recovered at higher shear rates as this plug is eroded by slip.

In accordance with the observation of a yield stress, yoghurt does show shear localisation in both the coneand-plate and cylindrical Couette geometries. While the appearance of a stress plateau at low shear rates suggests that a viscosity bifurcation might be occurring, 
signature partitioning into low and high shear rate bands according to the lever rule was not observed. This may be due to slip-highlighting the importance of slip on shear localisation/banding in soft glassy materials. From a material perspective, yoghurt can be considered to be a protein particle gel consisting of a weakly flocculated casein micelles network (with an initial volume fraction of casein micelles of approx. $10 \%$ ) embedded in a continuous phase composed of milk serum (mainly water containing lactose, globular proteins and minerals). The slip observed here may be associated with the formation of a serum-rich film produced by excluded volume interactions at the surfaces of the geometry (Yoon and McCarthy 2002). The correlation between the formation of a high shear rate band and the presence of slip tends to suggest that slip drives the erosion of the protein gel at low shear rates. While it seems physically reasonable that slip and slip-induced erosion of the gel should preferentially occur in the high stress region of the cylindrical Couette geometry, it remains unclear as to what triggers slip at the outer wall of the cylindrical Couette geometry at intermediate shear rates.

Acknowledgements The authors thank Aurélie Cucheval, Institute of Fundamental Sciences, Massey University, New Zealand for the confocal microscopy measurements. The NMR velocity measurements were performed in the Rheo-NMR Facility operated by Prof. Paul Callaghan at Victoria University of Wellington, New Zealand. Allan Raudsepp thanks the Royal Society of New Zealand for funding and Kirk Feindel thanks the Natural Sciences and Engineering Research Council of Canada for a post-doctoral fellowship.

\section{References}

Basak S, Ramaswamy HSA (1994) Simultaneous evaluation of shear rate and time dependency of stirred yogurt rheology as influenced by added pectin and strawberry concentrate. J Food Eng 21:385-393

Benezech T, Maingonnat JFA (1994) Characterization of the rheological properties of yoghurt. J Food Eng 21:447-472

Bicout D, Maret G (1994) Multiple light scattering in TaylorCouette flow. Physica A 210:87-112
Bicout D, Maynard R (1993) Diffusing wave spectroscopy in inhomogeneous flows. Physica A 199:387-411

Britton MM, Callaghan PT (1997) NMR Microscopy and the non-linear rheology of food materials. Magn Reson Chem 35:S37-S46

Callaghan PT (1991) Principles of nuclear magnetic resonance microscopy. Oxford University Press, Oxford

Callaghan PT (2008) Rheo-NMR and shear banding. Rheol Acta 47:243-255

de Kruif CG, Zhulina EB (1996) Kappa-casein as a polyelectrolyte brush on the surface of casein micelles. Colloids Surf A: Physicochem Eng Asp 117:151-159

Gibaud T, Barentin C, Manneville S (2008) Influence of boundary conditions on yielding in a soft glassy materials. Phys Rev Lett 101:258302

Gibaud T, Barentin C, Taberlet N, Manneville S (2009) Shear induced fragmentation of laponite suspensions. Soft Matter 5:3026-3037

Henningsson M, Ostergren K, Dejmek P (2006) Plug flow of yoghurt in piping as determined by cross-correlated dualplane electrical resistance tomography. J Food Eng 76:163168

Horne DS (1998) Casein interactions: casting light on the black boxes, the structure in dairy products. Int Dairy J 8:171177

Hoult DI, Richards RE (1975) Critical factors in the design of sensitive high resolution nuclear magnetic resonance spectrometers. Proc R Soc Lond A344:311-340

Lucey JA, Singh H (1998) Formation and physical properties of acid milk gels: a review. Food Res Int 30(7):529-542

Moller PCF, Rodts S, Michels MAJ, Bonn D (2008) Shear banding and yield stress in soft glassy materials. Phys Rev E 77: 041507

Olmsted PD (2008) Perspectives on shear banding in complex fluids. Rheol Acta 47:283-300

Raudsepp A, Callaghan PT, Hemar Y (2008) A study of the nonlinear rheology of complex fluids using diffusing wave spectroscopy. J Rheol 52:1113-1129

Rogers SA, Vlassopoulos D, Callaghan PT (2008) Aging, yielding and shear banding in soft colloidal glasses. Phys Rev Lett 100:128304

Salmon J-B, Collin A, Manneville S (2003) Velocity profiles in shear-banding wormlike micelles. Phys Rev Lett 90:228303

Suwonsichon T, Peleg M (1999) Rheological characterization of almost intact and stirred yogurt by imperfect squeezing flow viscometry. J Sci Food Agric 79:911-921

Weitz DA, Pine D (1992) Diffusing-wave spectroscopy. In: Brown W (ed) Dynamic light scattering. Oxford University Press, Oxford

Yoon WB, McCarthy KL (2002) Rheology of yogurt during pipe flow as characterized by magnetic resonance imaging. $\mathrm{J}$ Texture Stud 33:431-444 